\title{
Exciton dynamics in carbon nanotubes
}

\author{
Muhammad Tariq Sajjad
}

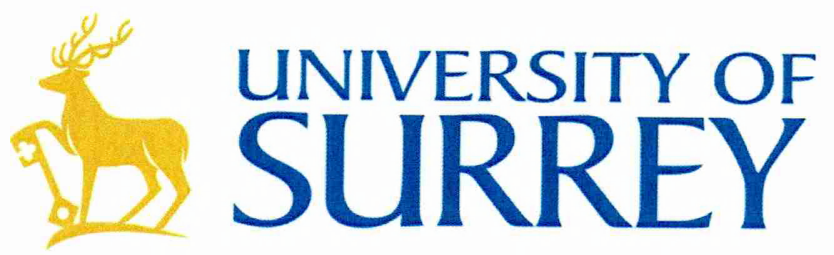

Submitted for the degree of Doctor of Philosophy

Advanced Technology Institute

Faculty of Engineering and Physical Sciences

University of Surrey

Guildford, Surrey, GU2 7XH, UK

October 2012 
ProQuest Number: 27721039

All rights reserved

INFORMATION TO ALL USERS

The quality of this reproduction is dependent upon the quality of the copy submitted.

In the unlikely event that the author did not send a complete manuscript and there are missing pages, these will be noted. Also, if material had to be removed, a note will indicate the deletion.

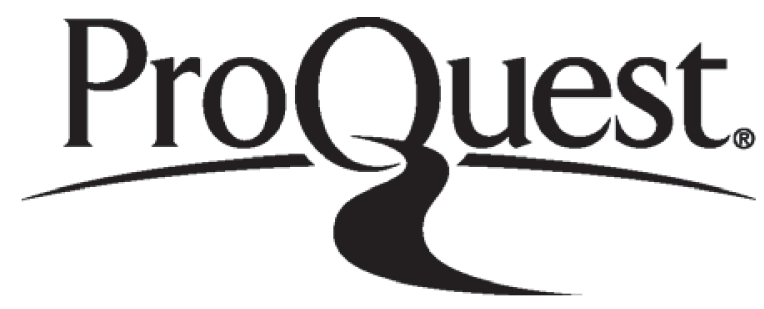

ProQuest 27721039

Published by ProQuest LLC (2019). Copyright of the Dissertation is held by the Author.

All rights reserved.

This work is protected against unauthorized copying under Title 17, United States Code Microform Edition (C) ProQuest LLC.

ProQuest LLC.

789 East Eisenhower Parkway

P.O. Box 1346

Ann Arbor, Ml $48106-1346$ 


\begin{abstract}
The confinement of excited charges in carbon nanotubes has significant effect on their optical and electronic properties. The absorption of light generates strongly correlated electron-hole pairs (excitons) in carbon nanotubes. We present a study of the decay of these photogenerated excitons in solutions of semiconducting SWNTs using the degenerate pump probe technique. Under specific experimental conditions, the exciton-exciton reactions on carbon nanotubes were found to correspond to an ideal 1D coalescence-diffusion system with distinct regions of reaction-limited and diffusion-limited behaviours. We provided the first experimental evidence for such a system of 'universal behaviour' at longer times which exhibits a power law decay whose exponent and amplitude are independent of the initial population - one of the key characteristics of this reaction-diffusion system. We also show for the first time that exciton-exciton interactions are long-range, and further that the transition between reaction-limited and diffusion-limited regimes is much more abrupt than is predicted by existing theories. A modified theory incorporating a finite reaction length provided an excellent fit to the experimental data for a reaction length of $\sim 7 \mathrm{~nm}$. We determined the reaction rate constant of $k_{r}=(3.76 \pm 0.04) n m / p s$ and the diffusion coefficient of $D=(8.1 \pm 0.4) \mathrm{nm}^{2} / p s$ from fitting of the asymptotic regimes with rate equations.

We also provided the first experimental evidence of sub-diffusive transport of excitons in quasi 1D SWNTs through studies of exciton annihilation dynamics in HiPco and CoMoCat SWNTs, where we observed that excitons in HiPco SWNTS exhibit normal diffusive transport where decay follows a $t^{-|\alpha|}$ power law with decay exponent $(\alpha \approx 0.5)$, whereas excitons in CoMoCat SWNTs decay more slowly with decay exponent $(\alpha \approx 0.3)$ as result of sub-diffusive transport. We correlate this slow decay to a higher defect concentration in CoMoCat SWNTs as compared to HiPco measured using Raman spectroscopy and X-ray photoelectron spectroscopy. The experimental results were also compared to results from a Monte Carlo simulation of 1D diffusion in a fluctuating potential landscape which shows good agreement with experiment and underlines the necessity to consider spatial aspects (separation of defects and interaction range) in theoretical approaches to the dynamics.

The study of excitonic decay in single walled carbon nanotubes is very important in terms of understanding of fundamental photophysics of 1D system and their possible application in electronics and photonic devices especially in light emitting devices and non-linear optics.
\end{abstract}




\section{ACKNOWLEDGEMENT}

First of all I would like to say thanks to my supervisor Prof. Jeremy Allam who placed tremendous faith in my abilities and has helped, directed and supported me in carrying out this interesting research. Without his support and cooperation this thesis would have not been possible.

I would like to extend my appreciation towards others in ATI especially Co-supervisor Dr. Richard Curry, and Dr. Konstantin Litvinenko, Dr Zhongyang Wang, Dr. Cristina Giusca for their useful help and valuable discussion throughout my $\mathrm{PhD}$ experimental work, and Christopher Buxey and John Underwood for technical help and Julie Fletcher for her support in administrative matters.

I am also thankful to our collaborator Dr Alan Dalton and his group especially Dr. Izabella Jurewicz at Physics department, University of Surrey, UK for sample supply and useful discussion on sample preparation and characterisation and Prof Tom Brown, Prof. Wei Loh, and Dr. Quan-Hong Yang from University of Southampton, UK for providing such a valuable sample. Also I would like to extend my appreciation towards all my colleagues in ATI especially Sofia Siddique, Zahida Batool, Marina Leontiadou, Muhammad Anas Razali, Ali Alshehri, Imalka Jayawardena, and Zeina Hashim for their benevolence and their cordiality.

I would also like to dedicate this thesis to my parents H. Sajjad Husain, Kaneez Fatima, father in law Babar Shah Khan, mother in law Abida Babar and finally to my wife Shumaila Babar and my son Arsalan Khan for being inspiration to successfully complete my thesis. I hope I have made all these people proud and happy by completing this project by the grace of Almighty God.

At the end I am grateful to EPSRC for providing funding for this project and ATI for financial support. 


\section{TABLE OF CONTENTS}

Introduction .17

2. Background Theory and Literature Review ...................................................20

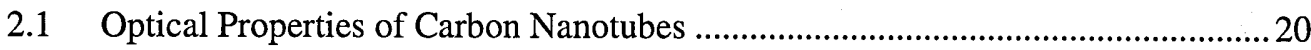

2.1.1 Basic Properties of Carbon Nanotubes ............................................................20

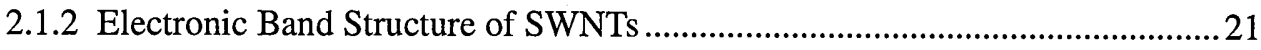

2.1.3 Density of States for Semiconducting and Metallic SWNTs ...........................25

2.1.4 Evidence of Excitons in Carbon Nanotubes ....................................................26

2.1.5 Linear Optical Absorption of SWNTs...........................................................28

2.2 Diffusive Transport of Excitons in Carbon Nanotubes ...........................................30

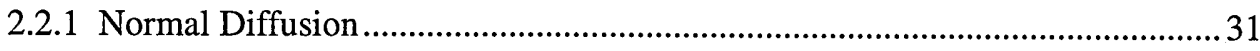

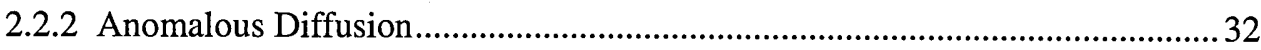

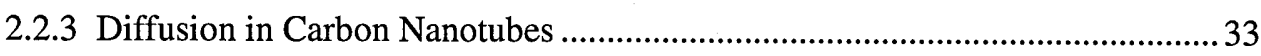

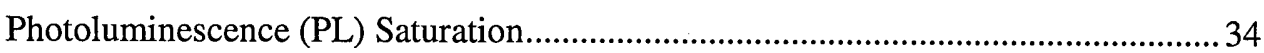

2.3 Reaction Kinetics: Reaction-limited and Diffusion-limited Processes ....................35

2.3.1 The 1D Single-species Bimolecular Annihilation and Coagulation Reaction ..36

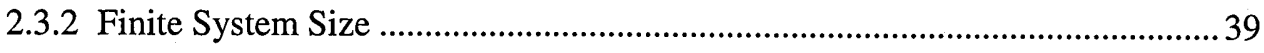

2.3.3 The 1D Reaction-Diffusion System with Both Finite Reaction and Diffusion Rate

2.4 Exciton-exciton Annihilation: Reaction-limited and Diffusion-limited Annihilation in Carbon Nanotubes

2.4.1 Exciton-exciton Annihilation in SWNTs Neglecting Transport Effects........... 42

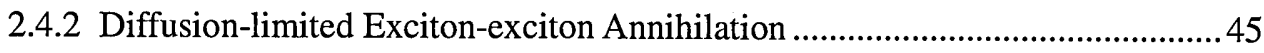

2.4.3 Reaction-limited and Diffusion-limited Annihilation in SWNTs ......................48

2.4.4 Effect of Trapping on Diffusive Transport in One Dimensional System including Carbon Nanotubes ..........................................................................4 49

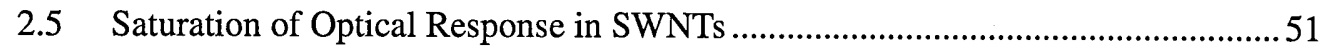

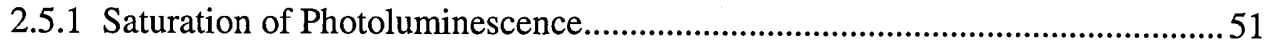

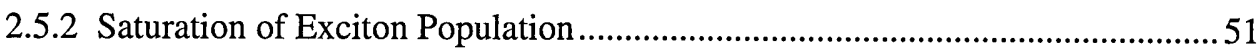

2.6 Plasmonic Contribution to Optical Response in Carbon Nanotubes ......................52

3. Experimental Techniques................................................................................54

3.1 The Amplified Titanium-doped Sapphire Ultrafast Laser System...........................54

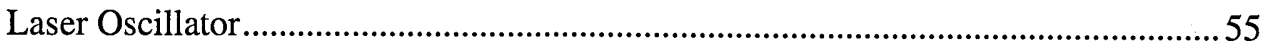

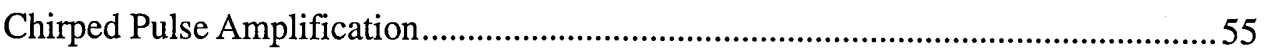

OPA (Optical Parametric Amplifier) .......................................................................56 
3.1.1 Definition of Beam and Pulse Parameters ......................................................56

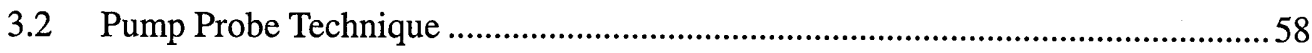

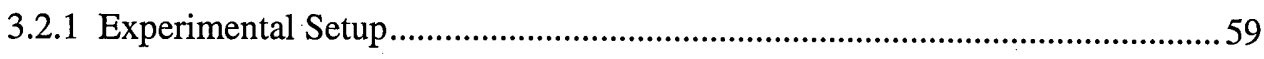

3.2.2 Quantitative Interpretation of Pump Probe Spectroscopy................................6 65

3.3 White Light Generation and Spectrally Resolved Pump Probe Measurements..... 66

4. Sample Details and Characterisation ....................................................................69

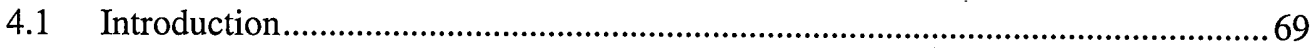

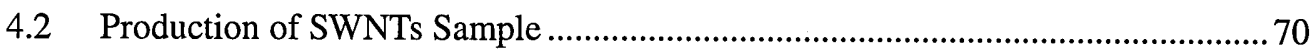

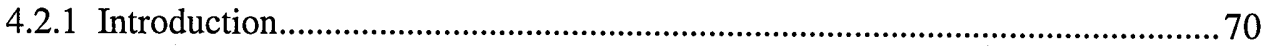

4.2.2 ss-DNA Wrapped HiPco SWNTS in Aqueous Suspension .............................. 70

4.2.3 CoMoCat SWNTs in Aqueous Suspension..................................................... 71

4.2.4 Structured CNT-Polymer Composite ............................................................... 72

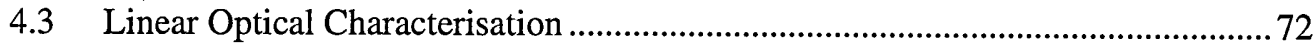

4.3.1 Absorption Measurement of ss-DNA HiPco SWNTs ……………...................73

4.3.2 Absorption Measurement of CoMoCat1 SWNT Sample ..................................77

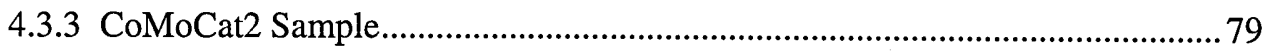

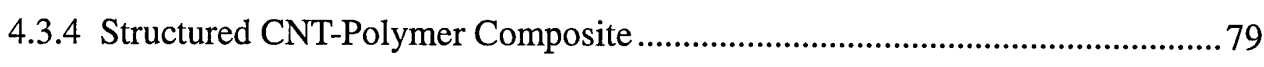

4.4 Length and Diameter Distribution Study of SWNTs by Atomic Force Microscopy (AFM) Measurements .................................................................... 80

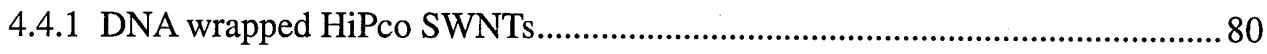

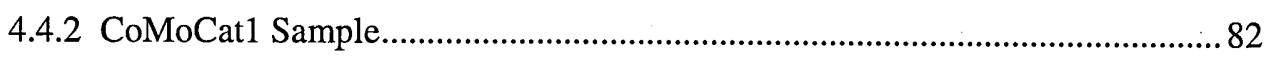

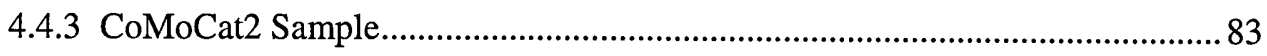

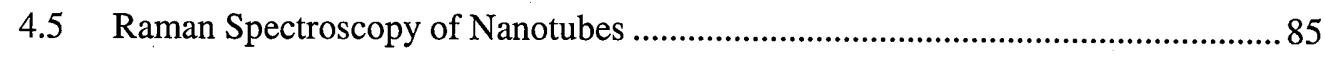

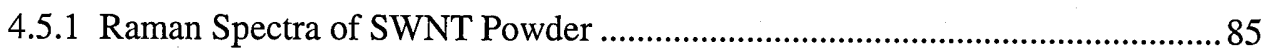

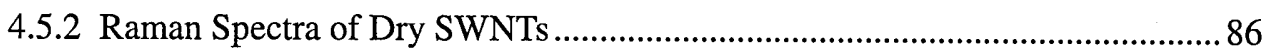

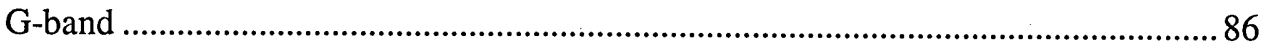

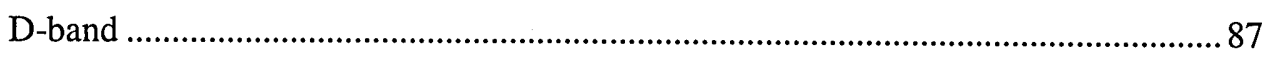

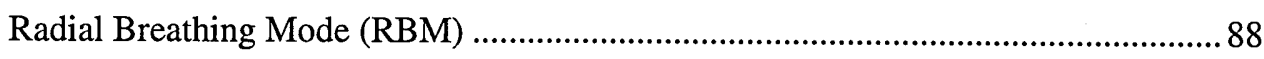

4.5.3 Raman Spectra of SWNTs Solution............................................................ 90

4.6 X-ray Photoelectron Spectroscopy (XPS) of SWNTs..........................................93

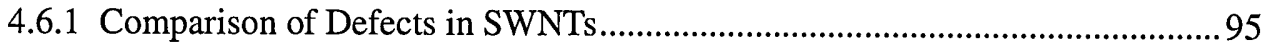

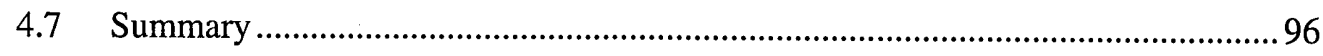

5. Pump Probe Measurements of HiPco SWNTS...............................................97

5.1 Experimental Results and their Qualitative Interpretation....................................98

5.2 Direct Determination of the Exciton Population.................................................105

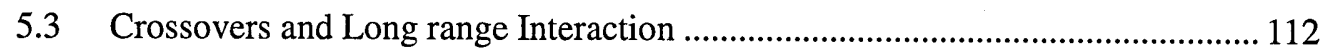




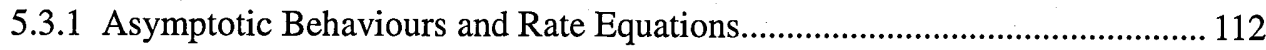

5.3.2 Reaction-Diffusion Crossover and the Interaction Range ............................ 113

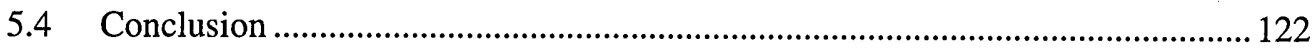

6. Pump Probe Measurements of CoMoCat SWNTs .........................................124

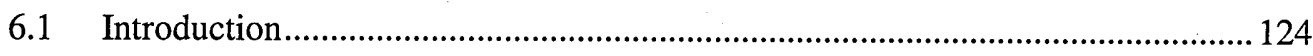

6.2 Pump Probe Measurements of CoMoCat SWNTs .............................................. 125

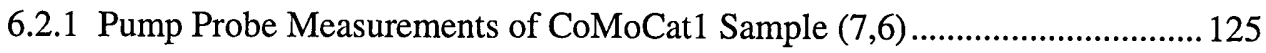

6.2.2 Pump Probe Measurements of CoMoCat2 SWNTs....................................... 132

6.3 Comparison of CoMoCat SWNTs with ss-DNA HiPco SWNTS ......................... 133

6.4 Interpretation of Pump Probe Results ................................................................. 134

6.4.1 Interpretation of Asymptotic Long Time Slow Decay and Subdiffusive

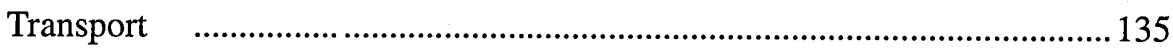

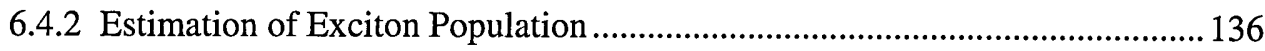

6.4.3 Determination of Reaction Rate Coefficients ............................................... 137

6.4.4 Simple Theoretical Model for Reaction Kinetics with Anomalous Reaction

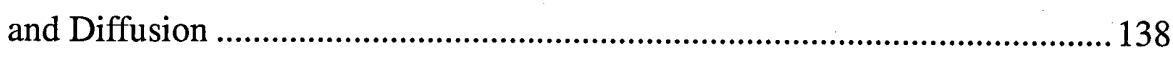

6.4.5 Comparison with Monte Carlo Simulation ................................................... 142

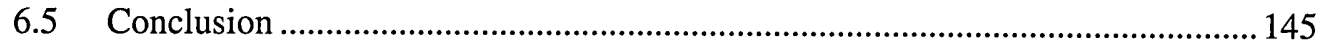

7. Conclusion and Future Works.......................................................................147

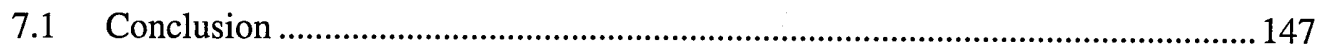

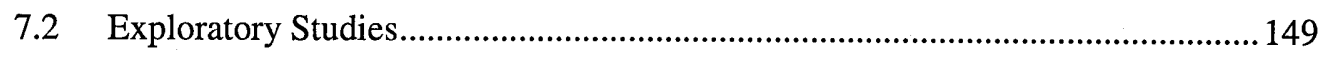

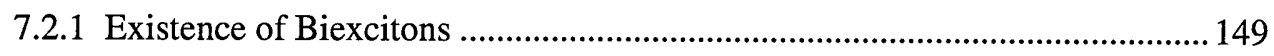

7.2.2 Spectrally Resolved Pump Probe Measurements: Energy Transfer Between

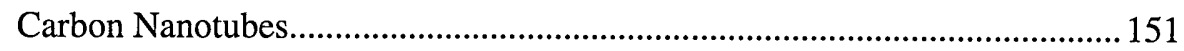

7.2.3 Annihilation Induced Heating..................................................................... 154

7.2.4 Measurement of Stretch-aligned Carbon Nanotubes ..................................... 156

7.2.5 Effects of Spatial Ordering on Early Reaction Kinetics - Detectability of the 'Exciton Crystal' ...................................................................................... 163

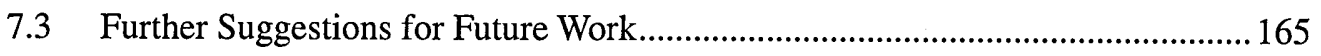

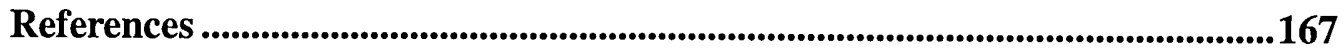




\section{LIST OF FIGURES}

Figure 2-1: The unrolled honeycomb of a $(4,2)$ nanotube, adapted from the ref [2]. The nanotube can be constructed by connecting the sites $\mathrm{P}$ and $\mathrm{Q}$, and $\mathrm{R}$ and $\mathrm{S}$. The vectors $\mathrm{PS}$ and $\mathrm{PQ}$ correspond to translational vector $\mathrm{T}$ and chiral vector $\mathrm{Ch}$ respectively. 20

Figure 2-2: (a) Arrangement of carbon atoms on the surface of single sheet of graphite (graphene layer), showing the periodic arrangements of unit cells with primitive lattice vectors a1 and a2. (b) Reciprocal lattice of graphene with basis vectors A1 and A2 along with first Brillion zone. This figure is taken from the ref [5].

Figure 2-3: The energy dispersion relations of graphene throughout the whole Brillion Zone. The inset figure illustrates the energy dispersion relation along the high symmetry directions, showing the degeneracy of $\pi$ band and $\pi *$ band at $\mathrm{K}$ points of reciprocal lattice of graphene. This figure is taken from [2].

Figure 2-4: $1^{\text {st }}$ Brillion zone of graphene lattice with straight lines obtained from the periodic boundary condition along the circumference of the nanotube taken from [5]. .24

Figure 2-5: Band structure and electronic density of states (van Hove singularities) for (a) metallic and (b) semiconducting SWNTS. This figure is adapted from [7]. .25

Figure 2-6: Schematic representation of single-particle states (left) and excitonic states (right) with excitation into $E_{22}$ and emission from $E_{11}$. This figure is taken from [15].

Figure 2-7: Schematic representation of one photon and two photon absorption in carbon nanotubes. (a) One photon absorption is linked to the excitonic states with odd (u) symmetry. (b) Two photon absorption couples with excitonic states with even (g) symmetry. In both cases, fluorescence occurs from the lowest one photon active $(1 \mathrm{u})$ state. This figure is taken from [18].

Figure 2-8: Contour plot of two photon excitation spectra of SWNTs taken from [11]. The one photon fluorescence emission is plotted as function of two photon excitation energy which clearly indicates the excitonic nature of SWNT optical transitions. .28

Figure 2-9: Linear absorption spectrum of a well isolated suspension of HiPco SWNTs, showing various sharp peaks with large background. The individual narrow peaks are due to interband transition for the specific $(n, m)$ species.

Figure 2-10: (a) Schematic of electronic density of states for semiconducting SWNTs showing the optical excitation of E22 and fluorescence emission from E11 (solid arrows). Dashed arrows in this figure denote the nonradiative relaxation before emission. (b) Contour plot of fluorescence of carbon nanotubes as function of excitation and emission wavelengths. This figure is taken from Bachilo [24]. 
Figure 2-11: Diffusive growth of 1D Gaussian distribution with time.

Figure 2-12: Theoretical prediction of population decay as a function of time, taken from [54]. The detailed description is presented in the text.

Figure 2-13: (a) Normalised time-resolved fluorescence intensity Vs time delay at different excitation intensities. (b) Maximum fluorescence intensity $\mathrm{I}_{0}$ and fitted parameter $\mathrm{N}(0)$ Vs excitation intensity. (c) Inverse of the normalised fluorescence intensity $\left(\mathrm{I}(\mathrm{t}) / \mathrm{I}_{0}\right)$ plotted against $\mathrm{t} 1 / 2$ for the highest excitation intensity. The dashed line is obtained by assuming $d \geq 2$ and dotted line for one dimension exciton diffusion. This figure is taken from [67]

Figure 2-14: (a) Max. Amplitude of transient absorption kinetics probed at $953 \mathrm{~nm}$ (open squares) and $660 \mathrm{~nm}$ (filled circle) as a function of pump fluence. Inset show same data measured at $953 \mathrm{~nm}$ but plotted on the scale of square root of pump intensity. This figure is taken from Ma [68]. (b) Pump probe data taken from Valkunas [69]....

Figure 2-15: One (inset), two (filled circle) and three (open circle) e-h pairs dynamics extracted from experimental data of Huang [70].

Figure 2-16: Pump probe data taken from Russo [71] on double logarithmic scale. (a) Mono, bi and tri exponential and power law $(t-1 / 2)$ functions were used for the fitting and claimed that power law fits very well as compared to exponential functions for $(8,6)$. (b) Similar behaviour was observed for $(7,6)$ nanotubes and inset shows that good fit in this case requires four exponential functions

Figure 2-17: Optical transients as function of pump probe delay taken from Zhu [73] . The dynamics of photobleaching and photoinduced absorption is fitted with power law.

Figure 2-18: Pump probe data up to $\sim 1$ ns taken from Sutton [74]. The straight red line is $t-1 / 2$ power law decay.

Figure 2-19:- (a) The non resonant pump probe measurement of SWNT taken from Lauret [84] is showing monoexponential decay of 1 ps. (b) optical absorption spectra of CoMoCat SWNTs obtained through different separation method. This picture is adapted from [88] .53

Figure 3-1: Schematic of ultrafast laser system. The brief description of each unit is given in the text. REGA = Regenerative amplifier, OPA = Optical parametric amplifier. .55

Figure 3-2: Pump probe trace as function of pump probe delay. The detail about the trace is given in text.

Figure 3-3: Experimental setup used to obtain the pump probe results presented in this thesis. BD is beam dump and L1, L2, L3, L4 are lens1, lens2, lens3, lens4 respectively..

Figure 3-4: Comparison of SNR for the case of unbalanced and balanced detector. Top graph was taken with unbalanced detector and bottom one with balanced detector.

Figure 3-5: Schematic of pump probe overlap (a) when the angle between pump and probe is 00 (b) when the angle is $\theta \sim 200$. The detail is given in text. 
Figure 3-6: Standard error in mean, appeared due to interference fringes of beams, was used to measure position of time zero and pulse width. Black dots are standard error in mean of individual pump intensities used, blue line is mean value of all standard error in mean, and red line is the fit using a Gaussian function.

Figure 3-7: Schematic of experimental setup of generation of white light continuum probe pulses. $\mathrm{VND}=$ variable neutral density filter, $\mathrm{VA}=$ variable aperture to adjust the beam profile, $\mathrm{FL}=10$ $\mathrm{cm}$ focussing lens, $\mathrm{CL}=5 \mathrm{~cm}$ collimating lens, $\mathrm{HPF}=$ high pass filter to remove the remnant 800 $\mathrm{nm}$ laser beam from the white light. Sapphire was mounted on translation stage with micrometer adjust in all three direction.

Figure 3-8: The optimised white light spectrum generated at the repetition rate of $100 \mathrm{kHz}$ using a 10 $\mathrm{cm}$ focussing lens. The spectrum is broad enough to probe the E11 transitions of SWNTs. 67

Figure 3-9: The experimental arrangements of spectrally resolved pump probe measurements. OPA = Optical Parametric amplifier, L1, L2, L3 are lens 1, lens2, lens3 respectively, BD is beam dump.

Figure 4-1: Absorption spectrum of ss-DNA wrapped HiPco SWNTs. The arrow corresponds to wavelength selected for the pump probe measurements presented in chapter 5 of this thesis...... 74 Figure 4-2: Background-subtracted absorption spectra of ss-DNA wrapped HiPco SWNTs along with a fit using multiple Lorentzians. Each peak is labelled corresponding to the specific nanotube species indicated in Table 4.1

Figure 4-3: Absorption spectrum of CoMoCat1 SWNTs. The numbers label the transitions which arise from interband transitions in both metallic and semiconducting nanotubes. .77

Figure 4-4: Background free absorption spectrum of CoMoCat1 SWNTs as function of energy (eV) fitted with Lorentzians. .78

Figure 4-5: Absorption spectrum of CoMoCat2 Sample (a) plotted as function of wavelength (b) as function of energy. The features in the E11 region are broader as compared to other samples discussed above, suggesting a greater degree of bundling. .79

Figure 4-6: Absorption spectrum of SWNT-polymer composite in latex film (a) plotted as a function of wavelength (b) as function of energy...... .80

Figure 4-7: AFM image of ss-DNA wrapped HiPco SWNTs. (a) AFM height profile image used to measure the length of nanotubes. (b) Phase mode used to identify the wrapping of DNA around the nanotube.

Figure 4-8: (a) Nanotube length histograms of ss-DNA wrapped HiPco SWNTs obtained from the AFM images shown in Fig. 4-7. (b) Nanotube length histograms fitted with LogNormal distribution.

Figure 4-9: AFM measurement of CoMoCat1 sample (a) diameter distribution (b) length distribution measured using the height profile of AFM images. The total number of SWNTs measured was 52. 
Figure 4-10: AFM images of CoMoCat2 SWNTs sample. The SWNTs in this sample are longer than previous samples studied. Also AFM images show that nanotubes are not straight which gives rise to defect centres for the excitons in the pump probe measurements discussed in chapter $6 . .83$ Figure 4-11: AFM height profile of CoMoCat2 SWNTs used to measure the diameter of nanotubes. 84 Figure 4-12: Diameter and length distribution of CoMoCat2 SWNTs sample, measured using the height profile of AFM images.

Figure 4-13: Raman spectra of SWNTs powder (a) HiPco powder (b) CoMoCat Powder. The Raman spectra show similar features for both SWNT samples.

Figure 4-14: Raman Spectra of dry SWNTs. (a) ss-DNA wrapped HiPco SWNTs (b) CoMoCat1 SWNT sample. The features observed are discussed in the text.

Figure 4-15: Radial breathing mode (RBM) bands of SWNTs (a) ss-DNA HiPco SWNTs (b) CoMoCat1 SWNTs.

Figure 4-16: Radial breathing mode (RBM) bands of SWNTs fitted with of Lorentzians (a) ss-DNA wrapped HiPco SWNTs (b) CoMoCat1 SWNTs.

Figure 4-17: Background-subtracted Raman spectra of SWNT solution showing the same features as observed in the SWNT powder.

Figure 4-18: Raman study of CoMoCat1 sample and HiPco SWNTs. ID / IG was compared to study the defect in these samples. The black line is the experimental data and red line is fit to the data using the combination of Gaussian and Lorentzian functions. The green lines represent the position of each fitted peak. .92

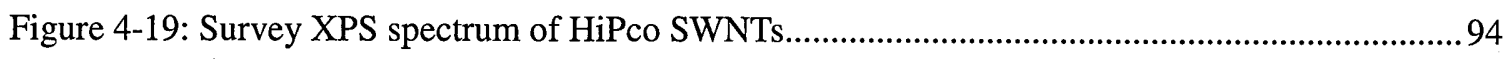

Figure 4-20: XPS survey of CoMoCat SWNTs, showing the different peaks....................................94

Figure 4-21: XPS C1s spectra of SWNTs fitted with three Gaussians (a) CoMoCat1 SWNTs in sodium cholate (b) ss-DNA wrapped HiPco SWNTs.

Figure 5-1: Differential transmission at high pump power of $26 \mathrm{~mW}$. The black dots are values of individual scans and red curves are the mean value of individual scans. The blue line is standard error in the mean whose peak value determined the time zero delay of pump and probe beam (a) linear transmission, (b) logarithmic of transmission, (c) log-log plot of normalised differential transmission. Error bars are combination of the standard error in the measured transmission and in the background. Dashed line indicates $t-1 / 2$ power law and is included here as guide to the eye.

Figure 5-2: Pump fluence dependent differential transmission as function of pump probe delay, showing saturation of signal at high pump fluences, and shift in peak position of signal from zero time delay with pump fluences, (a) differential transmission on linear scales (b) logarithmic of differential transmission.

Figure 5-3: Fluence dependent pump probe data as function of pump probe delay up to $1 \mathrm{ns,} \mathrm{dashed}$ 
line indicates the $t-1 / 2$ decay at long time. All the traces follow the $t-1 / 2$ power law at long time regardless of optical excitation densities. Deviation from this $t-1 / 2$ power law is seen around $\sim 1 \mathrm{~ns}$, where the decay becomes slow.

Figure 5-4: Logarithmic derivative of $S(\Delta \mathrm{T} / \mathrm{T} 0)$, equal to exponent ' $\alpha$ ' of a power law $t-\alpha$. The dashed line indicated the $t-1 / 2$ power law decay with decay exponent $\alpha=0.5$. 102

Figure 5-5: The derivative of $S=\Delta \mathrm{T} / \mathrm{TO}$ as function of $\mathrm{S}$ for ss-DNA wrapped carbon nanotubes sample, dashed lines indicate the region of $S^{2}$ and $S^{3}$.

Figure 5-6: Second logarithmic derivative ' $\beta$ ' of pump probe data plotted only for highest pump fluences up to $30 \mathrm{ps}$, clearly show the transition between two power law behaviours. 104

Figure 5-7: Differential transmission as function of pump probe delay for the pump powers between $10 \mathrm{uW}$ to $26 \mathrm{~mW}$. The solid lines are fits with Simon's formula. 106

Figure 5-8: (a) Maximum number of excitons created per nanotube, as a function of laser power. The exciton density saturates at $\sim 145$ excitons per nanotube due to Pauli Exclusion principle. (b) The fitted value of diffusion coefficient $D$ obtained from figure 5-7 plotted as function of laser power. Circles are values of diffusion coefficient D obtained from Simon's formula and solid line is a linear fit. 107

Figure 5-9: $\mathrm{N}-1$ as function of scaled time, where the parameters are determined from the fitting of experimental data (from figure 5-7) with Simon's equation 2.18. The red line is fit with Simon's equation using average nanotube length of $184 \mathrm{~nm}$. The blue line shows Simons equation convolved with the length distribution. The inset shows the distribution of nanotubes lengths measured with AFM fitted with LogNormal distribution.

Figure 5-10: Exciton number as a function of scaled time. The scaling is performed based on the fitted parameters of the Simon equation 2.18. The circled region shows universal behaviour, independent of excitation density. 110

Figure 5-11: Reaction rate is plotted against the exciton number N. The black lines are asymptotes of reaction-limited and diffusion-limited rate equations, included here as guide to eye.

Figure 5-12: Dynamic crossover between reaction-limited and diffusion-limited regimes. Reaction rate is plotted against exciton number, on a log-log scale. Blue line is conventional theory with short-range interactions. Green line is new theory with interaction range of $7 \mathrm{~nm}$.

Figure 5-13: Schematic of short range interaction and long range (dipole-dipole) interaction. $\mathrm{L}_{\mathrm{ex}}$ is exciton length and $r$ is interaction range. 118

Figure 5-14: Reaction order $\beta$ plotted as function of exciton number. Experimentally, transition (from $\beta=2$ to $\beta \sim 3$ ) is much more abrupt than predicted by conventional theory (green curve). The blue line is the new theory of eq 5.18 with a reaction range $r=7 \mathrm{~nm}$. The dashed lines indicate the asymptotic values of $\beta=2$ and $\beta=3$. Inset: predicted variation of effective reaction order for $r=0,1,2,5,10,20$ in units of lattice spacing. 
Figure 6-1: Pump probe data plotted on double logarithmic scale up to $\sim 1$ ns. The straight line indicates $t-0.30$ power law included as guide to eye.

Figure 6-2: Pump probe data at different pulse energies up to $\sim 1 \mathrm{~ns}$, plotted on double logarithmic axis. The dotted line indicates $t-0.30$ decay, included as a guide to the eye.

Figure 6-3: The logarithmic derivative of $S$ as function of pump probe delay. The dashed line corresponds to $\alpha=-0.30$ and dotted line corresponds to $=-0.5$. The red line is an average of all the data at different pump energies for $t>10 \mathrm{ps}$.

Figure 6-4: Time derivative of $S$ as function of $S$ plotted on double logarithmic scale. The dashed lines indicate regions of $S^{2}$ and $S^{4}$ and are included here for a guide to eye.

Figure 6-5: (a) Fluence dependent pump probe data of CoMoCat1 SWNTs taken at $1142 \mathrm{~nm}$, normalised at long time to show the universal behaviour. (b) Time derivative of $S$ as function of $S$ plotted on double logarithmic scale for highest 6 pulse energies, showing identical behaviour independent of initial excitation densities.

Figure 6-6: Pump fluence dependence of differential transmission at $t=0 \mathrm{ps}$ fitted with the model described in [133]. The black dots are experimental data, red line is fit obtained with saturation model, and the dotted line is a linear dependence included here as a guide to the eye.

Figure 6-7: Normalise value of signal at $\mathrm{t}=0 \mathrm{ps}$ (red line), $\mathrm{t}=100 \mathrm{ps}$ (black line) and ratio of $S(0) / S(100)$ is plotted as function of pump pulse energy. The detail is given in the text.

Figure 6-8: (a) Pump probe traces of CoMoCat2 taken at different pump fluences (b) decay exponent of power law obtained from the derivative of logarithmic data. The heavy line is an average of the three curves for $t>10 \mathrm{ps}$. The dotted lines are guides to the eye.

Figure 6-9: Normalised pump probe data taken on ss-DNA wrapped HiPco and CoMoCat SWNTs at highest pump fluence. Smaxis maximum value of signal $S=\Delta T / T 0$ measured at $t=0$. The dotted lines are included as a guide to the eye. The curves are shifted vertically with respect to each other for the comparison.

Figure 6-10: (a) Logarithmic derivative which is equal to exponent $\alpha$ as function of time for three different nanotubes samples. The dashed lines are drawn as a guide to the eye. (b) Linear derivative of $\mathrm{S}$ as a function of $\mathrm{S}$. A line with gradient $\mathrm{n}$ corresponds to rate equation $d S d t=S n$, showing three distinct dependence at low exciton density. Curves are normalised for comparison.

Figure 6-11: Time derivative of data for sample CoMocat1 with $100 \mathrm{~nJ}$ pump energy. The three different regimes are clearly seen in the figure. Straight lines corresponding to these different regimes are included as a guide to the eye.

Figure 6-12: Schematic of defective nanotube; $r$ is the interaction range, $\mathrm{x}$ is the average distance between excitons, and $s$ is the separation between defects.

Figure 6-13: Schematic of nanotubes with defects showing three different cases: (1) reaction-limited 
process when separation between excitons is less than their interaction range, (2) normal diffusion when the their separation is greater than interaction range but less that the defect separation, and (3) subdiffusion when the separation between excitons is greater than the defect separation.

Figure 6-14: Experimental data fitted piecewise with equation 6.6. Symbols are experimental data; red line is fit to data using eq.6.6. The vertical dotted lines show the three different regions used for piecewise fitting.

Figure 6-15: Experimental data fitted with equation 6.6 with the same parameters used in Fig. 6-14. Symbols are experimental data, blue line is a fit with short range interactions, green line is fit without subdiffusion, and red line is fit achieved without normal diffusion

Figure 6-16: Monte Carlo simulation of random walk on 1D lattice (figure provided by A. Moretti and J. Allam) 142

Figure 6-17: Results of Monte Carlo simulation performed on 1D lattice. (a) Exciton population is plotted as function of time. (b) Decay exponent " $\alpha$ " of power law which shows that reduction of the decay exponent is observed at sufficient defect density. (Figure provided by A. Moretti and J. Allam). 143

Figure 6-18: (a) The mean square displacement of the diffusing particle as a function of time which exhibits sublinear behaviour at sufficient defect density. (b) The decay exponent is plotted as a function of defect spacing (figure provided by A. Moretti and J. Allam).

Figure 6-19: Diffusion exponent $(\gamma / 2)$ plotted against reaction exponent $(\alpha)$, red line is linear fit with slope $\sim 1$

Figure 7-1: The signature of a biexciton assuming resonant pumping conditions and different values of inhomogeneous broadening, taken from Pedersen [136]

Figure 7-2: Spectrally resolved measurements of ss-DNA wrapped HiPco SWNTs plotted as function of wavelength. The sample was pumped at $1132 \mathrm{~nm}$ and probed with white light over the range of $1050-1350 \mathrm{~nm}$.

Figure 7-3: (a) Transient absorption spectrum of ss-DNA wrapped SWNTs in aqueous suspension as a function of wavelength for range of probe delays, (b) 2D contour plot of the spectrally resolved measurements

Figure 7-4: Dynamical response of HiPco SWNTs obtained through the vertical cut of Figure 7-3 (a). The dashed line shows a $t-1 / 2$ decay.

Figure 7-5: (a) Differential transmission for 4 different laser repetition rates. The laser intensity was adjusted to give approximately constant peak pulse amplitude. Pump intensity at the highest repetition rate was $12 \mathrm{~mW}$. (b) Same data but for a reduced average laser intensity. 155

Figure 7-6: Transmission electron micrographs $(a, c)$ and phase contrast atomic force micrographs $(b, d)$ of unstretched $(a, b)$ and $100 \%$ strained $(c, d)$ samples. Figure taken from Jurewicz et al. 
[96].

Figure 7-7: (a) Pump probe measurement of anisotropy of saturable absorption and its decay in a stretched sample [150\%]. The differential transmission is maximum in the case of parallel orientation of nanotubes with laser beams (red curve) and minimum when they are orthogonal (green curve). (b) Log-Log plot of the photoexcitation, which decays according to a $t-1$ power law (dashed line). Inset shows the reaction rate which is proportional to the square of the exciton population (dashed line).

Figure 7-8: (a) Pump probe measurement of a stretched sample at 3 different locations. (b) Data normalised at delay time of 2 ps. Inset: derivative of differential transmission plotted against differential transmission on a log-log scale. The gradient is the reaction order which is equal to 2 and 3 (dashed lines) at high and low density regimes respectively. 158

Figure 7-9: The decay exponent $\alpha$ for pump beam intensities of $3 \mathrm{~mW}$ and $10 \mathrm{~mW}$. The $\quad 3 \mathrm{~mW}$ data is the average of the 3 curves shown in Fig. 7-8 (a). 159

Figure 7-10: (a) Pump-probe transients taken on unstretched sample before (16/7/2010) and after (20/7/2010) multiple stretches by $150 \%$. (b) Transient response of stretched (red crosses, data from Fig. 7.8 (a)) and unstretched (blue squares; data from Fig. 7-10(a)) samples, normalised at a delay of $1 \mathrm{ps}$. The time axis is shifted to account for the initial condition. 161

Figure 7-11: Pump probe data taken on drop-cast SWNT-polymer (a) Linear plot showing fast initial decay followed by a long-lived negative signal. Inset: log-linear plot of the first ps, indicating an approximately exponential decay. (b) Absolute value of differential transmissivity, fitted to a biexponential decay with a constant offset. 162

Figure 7-12: (a) Measured reaction rates for different photoexcitation levels. The curves have been shifted laterally for clarity. (b) Monte Carlo calculation of the reaction rate for random and spatially-ordered initial states. 164 


\section{LIST OF TABLES}

Table 4-1: The (n,m) species present in aqueous solution of ss-DNA wrapped HiPco SWNTs along with $1^{\text {st }}$ and $2^{\text {nd }}$ transitions, width of Lorentzian peaks used in the fitting, and corresponding peak

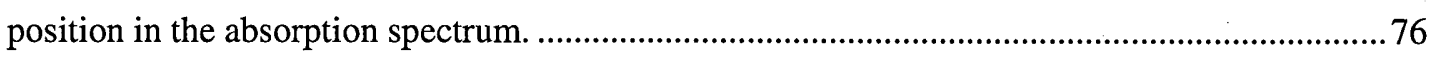

Table 4-2: The (n,m) species present in aqueous solution of CoMoCat1 SWNTs along with $1^{\text {st }}$ and $2^{\text {nd }}$ transitions, width of Lorentzian peaks used in the fitting, and corresponding peak position in the absorption spectrum shown in Fig. 4-4

Table 4-3: Average nanotubes length, standard deviation, and standard error in mean calculated from AFM data. .85

Table 4-4: Assignment of RBM peaks to SWNT species which were excited during Raman spectroscopy. .89

Table 4-5: Diameter of nanotubes determined from $\mathrm{G}^{-}$band frequency using equation 4.4. .92

Table 4-6: Comparison of ratio of D band to $G$ band for ss-DNA HiPco and CoMoCat SWNTs. Here $A$ is area and $I$ is intensity of fitted peak. .93

Table 4-7: FWHM and area obtained from C1s peaks of HiPco and CoMoCat1 SWNTs, showing that area as well FWHM of $\mathrm{sp}^{3}$ peak of CoMoCat is higher than HiPco which indicates that CoMoCat nanotubes have more defects. .96

Table 6-1: Comparison of SWNT samples prepared through different synthesis methods. 146 


\section{ABREVIATIONS}

\begin{tabular}{|c|c|}
\hline SWNT & Single-walled Carbon Nanotube \\
\hline SWNTs & Single-walled Carbon Nanotubes \\
\hline $\mathrm{T}$ & Translational Vector \\
\hline $\mathrm{C}_{\mathrm{h}}$ & Chiral Vector \\
\hline DOS & Electronic Density of States \\
\hline LUMO & Lowest Unoccupied Molecular Orbital \\
\hline HOMO & Highest Occupied Molecular Orbital \\
\hline SDS & Sodium Dodecyl Sulfate \\
\hline SDBS & Sodium Dodecyl Benzene Sulfonate \\
\hline $\mathrm{SC}$ & Sodium Cholate \\
\hline PL & Photoluminescence \\
\hline PLE & Photoluminescence Excitation Spectroscopy \\
\hline PAA & Poly Acrylic Acid \\
\hline AFM & Atomic Force Microscopy \\
\hline XPS & X-ray Photoelectron Spectroscopy \\
\hline $\mathrm{H} \& \mathrm{M}$ & Hoyuelos and $\mathrm{Ma}$ \\
\hline $\mathrm{ZBA}$ & Zhong and Ben-Avraham \\
\hline IPDF & Inter Particle Distribution Function \\
\hline $\mathrm{PFO}$ & Poly (9,9-dioctylfluorenyl-2,7-diyl) \\
\hline FWHM & Full Width at Half Maximum \\
\hline CPA & Chirped Pulse Amplification \\
\hline OPA & Optical Parametric Amplifier \\
\hline CPA & Chirped pulse amplification \\
\hline REGA & Regenerative amplifier \\
\hline $\mathrm{p}$ & Reaction probability \\
\hline $\mathrm{k}_{\mathrm{r}}$ & Reaction rate constant \\
\hline $\mathrm{k}_{\mathrm{d}}$ & Diffusion rate constant \\
\hline $\mathrm{D}$ & Diffusion coefficient \\
\hline $\mathrm{D}_{\gamma}$ & Subdiffusion coefficient \\
\hline $\mathrm{k}_{\gamma}$ & Subdiffusion rate constant \\
\hline SNR & Signal to Noise ratio \\
\hline
\end{tabular}




\section{INTRODUCTION}

This thesis describes experimental studies which show that exciton-exciton reactions in carbon nanotubes represent a near-ideal one-dimensional reaction-diffusion system. We verify experimentally for the first time some long-standing theoretical predictions for the properties of archetypal reaction-diffusion systems, as well as other dynamical properties which have not been predicted.

Single-walled carbon nanotubes (SWNTs) attracted considerable attention as a model 1D system since their discovery in 1993 [1]. SWNTs have unique physical properties including strong one-dimensional confinement of electrons and holes and formation of stable excitons [2] with much larger binding energies ( $400 \mathrm{meV}-1 \mathrm{eV})$. The diffusion of excitons is expected to be one-dimensional along the length of nanotubes due to large exciton size (1-2 $\mathrm{nm})$ than tube diameter. SWNTs can withstand high intensity without damage and posses strong absorption bands in a technologically relevant range (near infrared). Recently electronic and optical properties of carbon nanotubes have been intensively investigated from both a fundamental physics viewpoint and due to their possible application in optoelectronic devices. This has provided a basic knowledge of their structure and many of their properties both electronic and optical, but there are still many gaps and unanswered questions to be addressed. The main aim of this work is to understand the fundamental photophysics of 1D system specifically exciton-exciton processes in SWNTs because of the possibility of their application in the electronic and photonic devices especially in the light emitting devices and non-linear optics.

SWNTs are ideal 1D system to study the exciton photophysics from both theoretical and experimental viewpoints. Quantum confinement is expected to enhance particle-particle interactions, and evidence for an Auger-like exciton decay process has been found from both experimental results of pump probe and transient photoluminescence (PL). In order to understand the exciton-exciton interaction properly, one has to consider the 1D transport of excitons along length of SWNTs.

Since the first report in 2004 [3], there have been numerous reports (described in chapter 2) of non-radiative exciton decay in SWNTs with a wide range of reported behaviours and interpretations. The role of exciton transport, and the degree to which the behaviour is governed by intrinsic or extrinsic factors, are both contested. One aim of this work is to provide a clear discussion on the detailed mechanism of exciton-exciton interactions in the 
presence of exciton transport, and to identify when intrinsic or extrinsic effects are dominant.

In this work we used ultrafast spectroscopy which is a powerful tool to study the various dynamical processes in complex systems. By exciting a system with tuneable, intense, ultra short pulses, it is possible to follow various dynamical processes in these systems. The excitation dynamics in SWNTs predominantly focussed to population relaxation of electronic excited states.

In Chapter 2, a background theory and literature review is presented. In the first section of this chapter, we summarise the essential properties of SWNTs relevant to this thesis, which include band structure, density of states, linear optical absorption, and evidence for exciton formation upon optical excitation in SWNTs. We explain how the linear optical properties of SWNTs are linked with their molecular structure. We then address the one-dimensional diffusion of excitons in SWNTs, and summarise the wide range of values of diffusion coefficients which have been reported using different experimental methods.

In the third section of chapter 2, we discuss the bimolecular single-species coalescence reaction $X+X \rightarrow X$ in systems of different dimensionality. This reaction has been proposed as a model for exciton-exciton annihilation in SWNTs. We identify regimes where the decay rate follows the classical law of mass action, and other regimes (of dimensionality and density) where an anomalous decay rate holds. The relation of this anomalous decay to selforganised criticality and universality are briefly discussed. Finally, we describe recent literature on exciton dynamics in SWNTs which is closely related to our work. We address the inconsistencies of experimental results and interpretations which show that further work is required in this area. We also discuss how the presence of defects affects exciton transport and hence the anomalous decay dynamics.

Chapter 3 describes experimental tools and techniques used in obtaining results presented in this thesis. A general description of Ti-Sapphire ultrafast laser system is followed by the description of the pump probe technique. We focus on the specific modifications to the standard techniques which have been made to yield the results presented in this thesis. In the last section, a brief description of white light continuum generation, used as a probe for spectrally resolved measurements is given.

Chapter 4 describes the preparation, processing, and characterisation of the samples used in this thesis. A discussion of methods of isolation of SWNTs is followed by a description of their linear optical characterisation by means of which each peak observed in the linear 
absorption spectra is assigned to a specific SWNT species. The length and diameter of SWNTs measured with Atomic Force Microscopy (AFM) is then reported. In the last section of this chapter, Raman spectroscopy and X-ray photoelectron spectroscopy (XPS) are used to compare the defect density in different SWNT samples.

In chapter 5, we report intensity-dependant pump probe measurements taken on HiPco SWNTs which are wrapped in single-strand DNA to assist aqueous suspension. On the basis of these results, we describe SWNTs as an archetypal reaction-diffusion system in which the annihilation of excitons in limited either by their intrinsic reaction rate or by diffusion along the length of nanotube, depending on the density of excitons. A detailed investigation of the cross-over from reaction-limited to diffusion-limited regimes provides evidence for a longrange interaction between excitons. A simple theory is presented which reproduces the main experimental observations.

Chapter 6 presents a comparison of exciton decay dynamics in SWNTs which are synthesized by different methods, and which exhibit a different density of defects. The asymptotic dynamics provide evidence for a transition from diffusive to sub-diffusive transport of excitons on SWNTs. This interpretation is supported by Monte Carlo simulation of particles on a 1D lattice in the presence of potential barriers. The analytical theory described in chapter 5 is further modified to reproduce the exciton decay for sub-diffusive transport.

Chapter 7 summarises the main results of the thesis, and then presents proposals for future work, including some areas of work which have been initiated here but not fully concluded. 


\section{CHAPTER 2}

\section{BACKGROUND THEORY AND LITERATURE REVIEW}

\subsection{Optical Properties of Carbon Nanotubes}

\subsubsection{Basic Properties of Carbon Nanotubes}

Carbon nanotubes can be visualised as a single sheet of graphene rolled into cylindrical shape to form a nanotube. The unrolled honeycomb lattice of nanotube is shown in Fig. 2-1. The carbon nanotube is notionally constructed by rolling the honeycomb sheet in such a way that points $\mathrm{P}$ and $\mathrm{Q}$ overlap, and points $\mathrm{R}$ and $\mathrm{S}$ overlap. The vector $\overrightarrow{\mathrm{PS}}$ defines the translational vector " $\mathrm{T}$ " which represents the length of unit cell in the direction of nanotube axis and vector $\overrightarrow{\mathrm{PQ}}$ correspond to the chiral vector " $C_{h}$ " [2].

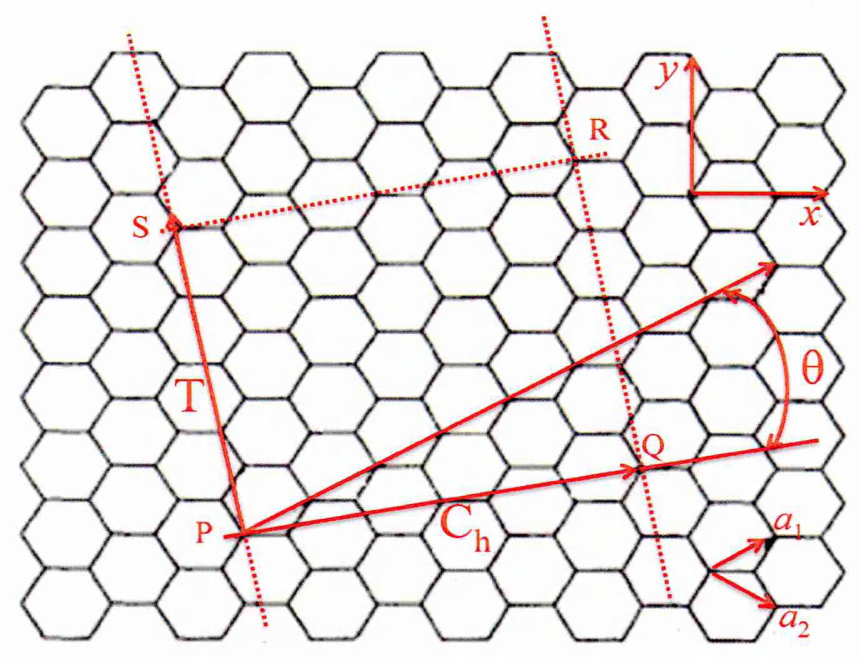

Figure 2-1: The unrolled honeycomb of a $(4,2)$ nanotube, adapted from the ref [2]. The nanotube can be constructed by connecting the sites $P$ and $Q$, and $R$ and $S$. The vectors $\overrightarrow{P S}$ and $\overrightarrow{P Q}$ correspond to translational vector $T$ and chiral vector $C_{h}$ respectively.

Carbon nanotubes are of two types, achiral carbon nanotubes (zigzag and armchair nanotubes) whose mirror images are identical and chiral nanotubes which exhibit spiral symmetry and whose mirror images cannot be superimposed. The structure of the nanotubes is described by the chiral vector $C_{h}$ which corresponds to a section perpendicular to the nanotube axis and is expressed by the real space primitive unit vectors $a_{1}$ and $a_{2}$ of the 
hexagonal lattice

$$
C_{h}=n a_{1}+m a_{2}=(n, m) .
$$

where $n, m$ are integers $(0 \leq m \leq n)$ and real space basis vectors are

$$
\begin{aligned}
& a_{1}=\left(\frac{3 a_{0}}{2}, \frac{\sqrt{3} a_{0}}{2}\right) . \\
& a_{2}=\left(\frac{3 a_{0}}{2}, \frac{-\sqrt{3} a_{0}}{2}\right) .
\end{aligned}
$$

where $a_{0}=1.42 A^{o}$ is the graphene lattice constant.

The $(n, n)$ nanotubes are armchair nanotubes and $(n, 0)$ nanotubes are zigzag nanotubes, and their names (zigzag and armchair) come from the pattern of atoms around the circumference of a SWNT. The diameter of the carbon nanotube is given

$$
d_{n}=\frac{a}{\pi} \sqrt{n^{2}+m^{2}+n m} .
$$

where $a=\sqrt{3} * a_{0}=2.46 A^{\circ}$.

The chiral angle $\theta$ specifies the spiral symmetry and is defined as the angle between the chiral $C_{h}$ and real space unit vector $a_{1}$ as shown in Fig. 2-1 and can be determined by

$$
\cos \theta=\frac{2 n+m}{2 \sqrt{n^{2}+m^{2}+n m}}
$$

The value of $\theta$ lies in the range of $0 \leq \theta \leq 30^{\circ}$ for chiral nanotubes, whereas $\theta=0^{\circ}$ in the case of zigzag nanotube and $\theta=30^{\circ}$ for the armchair nanotubes [2, 4].

\subsubsection{Electronic Band Structure of SWNTs}

The electronic band structure of carbon nanotubes derives from that of the graphene material it is composed of, modified by the additional periodicity of the tubular structure and the curvature of the graphene sheet. It therefore depends on the diameter and orientation of the carbon hexagon in honeycomb lattice relative to the nanotubes axis. 


\subsubsection{Reciprocal Lattice of Graphene}

The arrangements of the carbon atoms on the surface of graphene layer are depicted in Fig. 2-2 (a) showing the periodic arrangements of unit cells with primitive lattice vectors $a_{1}$ and $a_{2}$, and (b) the reciprocal lattice of the graphene with basis vectors $A_{1}$ and $A_{2}$ which are found from the real space primitive lattice vectors via [5]

$$
\begin{aligned}
& \overrightarrow{A_{1}}=\frac{2 \pi\left(\overrightarrow{a_{2}} \times \overrightarrow{a_{3}}\right)}{\overrightarrow{a_{1}} \cdot\left(\overrightarrow{a_{2}} \times \overrightarrow{a_{3}}\right)} \\
& \overrightarrow{a_{2}}=\frac{2 \pi\left(\overrightarrow{a_{3}} \times \overrightarrow{a_{1}}\right)}{\overrightarrow{a_{2}} \cdot\left(\overrightarrow{a_{3}} \times \overrightarrow{a_{1}}\right)}
\end{aligned}
$$

where $\overrightarrow{a_{3}}$ is unit vector in $\mathrm{z}$-direction. Using the definition of equation $2.2 \mathrm{a}$ and $2.2 \mathrm{~b}$, the basis vectors of reciprocal lattice become

$$
\begin{aligned}
& A_{1}=\left(\frac{2 \pi}{3 a_{0}}, \frac{2 \pi}{\sqrt{3} a_{0}}\right) \\
& A_{2}=\left(\frac{2 \pi}{3 a_{0}}, \frac{-2 \pi}{\sqrt{3} a_{0}}\right)
\end{aligned}
$$

"The first Brillion zone for the allowed K- vectors is obtained by drawing the perpendicular bisectors of the lines joining the origin $(0,0)$ to the neighbouring points on the reciprocal lattice" [5] and is shown in Fig. 2-2 (b) with shaded region.
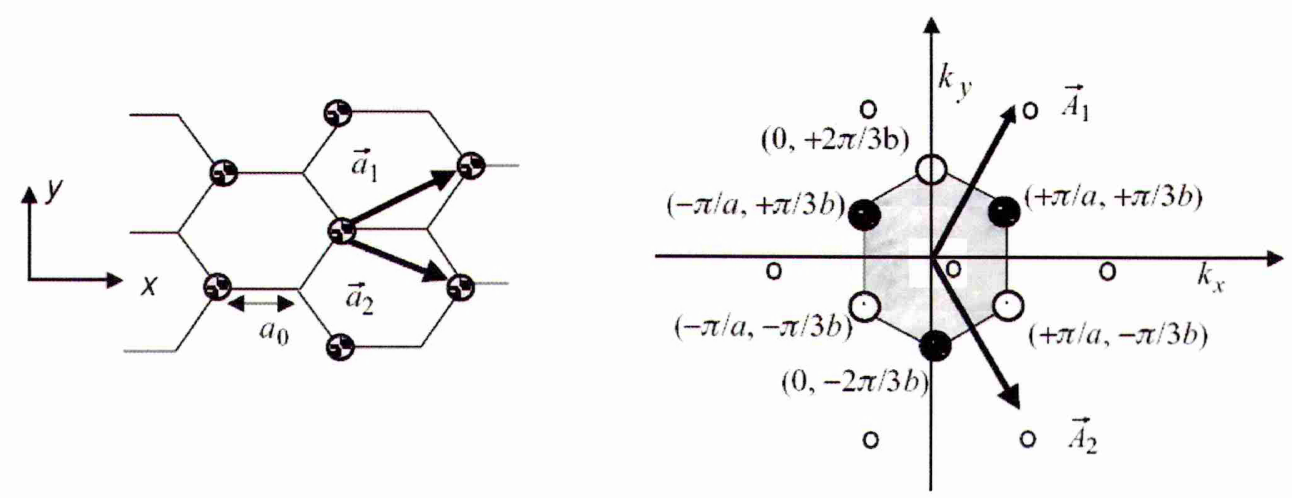

Figure 2-2: (a) Arrangement of carbon atoms on the surface of single sheet of graphite (graphene layer), showing the periodic arrangements of unit cells with primitive lattice vectors $a_{1}$ and $a_{2}$. (b) Reciprocal lattice of graphene with basis vectors $A_{1}$ and $A_{2}$ along with first Brillion zone. This figure is taken from the ref [5]. 


\subsubsection{Energy Dispersion of Graphene}

The conduction and valence band states of two-dimensional graphene can be described quite well by a tight binding model which uses the $2 p_{z}$ orbital (which is perpendicular to graphene plane and makes $\pi$ covalent bonds responsible for the electrical conduction) because all other orbitals are either far below or far above the Fermi energy. Hence using this model, the energy dispersion relation of graphene can be found by summing over any unit cell and all its neighbouring unit cells. The resultant relation is given by [5]

$$
E \sim \pm \sqrt{1+4 \cos _{y} b \cos k_{x} c+4 \cos ^{2} k_{y} b}
$$

where $c=3 a_{o} / 2$ and $b=\sqrt{3} a_{o} / 2$

From the energy dispersion relation given in equation 2.5, two values of $E$ can be obtained for each value of $K$ which results in two branches in the $E(K)$ plot i.e. $\pi^{*}$ antibonding (conduction band) at higher energies and $\pi$ bonding (valence band) at lower energies as shown in Fig. 2-3. The Fermi energy passes through the $K$ points of the reciprocal lattice of graphene at which these upper half $\pi^{*}$ band and lower half $\pi$ band are degenerate. Since the density of states is zero at the Fermi level, graphene is a zero-gap semiconductor $[2,5]$.

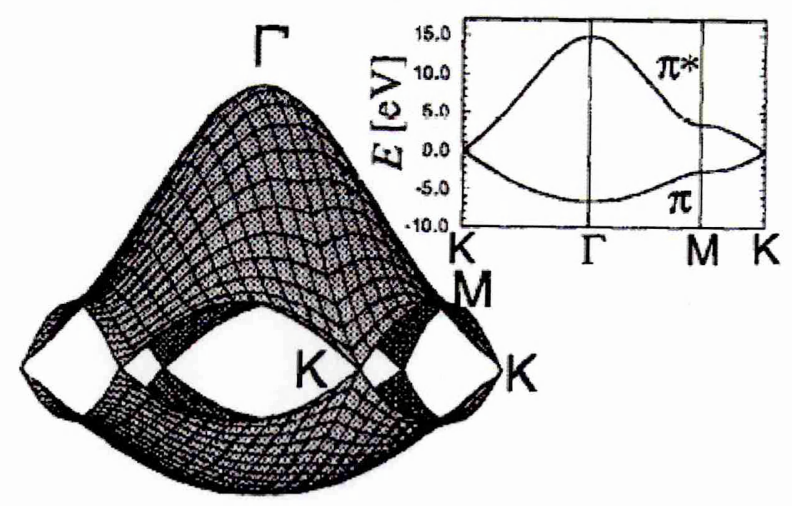

Figure 2-3: The energy dispersion relations of graphene throughout the whole Brillion Zone. The inset figure illustrates the energy dispersion relation along the high symmetry directions, showing the degeneracy of $\pi$ band and $\pi^{*}$ band at $K$ points of reciprocal lattice of graphene. This figure is taken from [2]. 


\subsubsection{SWNTs Band Structure from the Graphene}

As discussed above that the energy dispersion relation for graphene can be obtained using simple tight binding model which gives two bands: the $\pi^{*}$ antibonding (conduction) and the $\pi$ bonding (valence). Fermi energy is located at $E=0$, where these two bands $\pi^{*}$ and $\pi$ are degenerate at $K$ points of the reciprocal lattice of graphene (shown in Fig. 2-3). The values of $K$ at which $E=0$ can be found by setting the R.H.S of equation 2.5 to zero which gives solutions at the six corner of the Brillion zone as shown in Fig. 2-2 (b).

For SWNTs, the allowed values of $K$ can be obtained by imposing the periodic boundary condition in the circumferential direction which leads to the quantisation of wave vector around the circumference (chiral vector $C_{h}$ direction) of the nanotube, whereas the wave vector along the nanotube axis remain continuous due to large aspect ratio (considering the nanotube as infinite long cylinder) $[2,5]$. This periodic boundary condition in the circumferential direction is expressed as

$$
\vec{k} \cdot \vec{c}=k_{c}|c|=k_{x} a(m+n)+k_{y} b(m-n)=2 \pi t .
$$

The equation 2.6 shows that each integer value of $t$ corresponds to a straight line, resulting in a series of parallel lines for different integer values of $t$ as shown in Fig. 2-4. The dispersion relation of SWNTs can be obtained along any of these lines from cross-sectional cutting of the energy dispersion of graphene [6]. The carbon nanotubes will be metallic if any of these lines passes through the centre of one of the valleys e.g. $\left(k_{x} a, k_{y} b\right)=(0,2 \pi / 3)$ and is possible only if $\frac{(m-n)}{3}=t$ (according to equation 2.6).

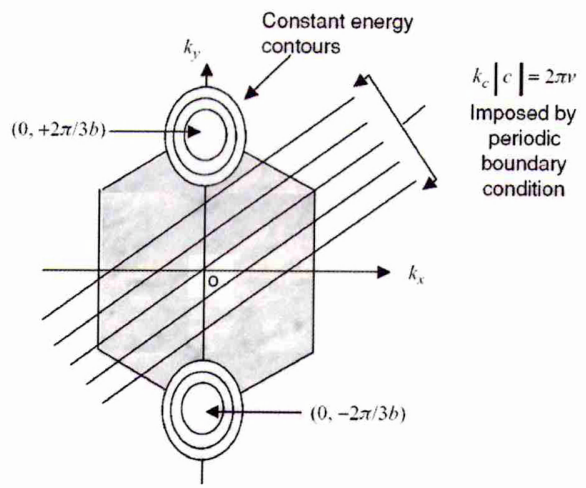

Figure 2-4: $1^{\text {st }}$ Brillion zone of graphene lattice with straight lines obtained from the periodic boundary condition along the circumference of the nanotube taken from [5]. 
Hence the carbon nanotubes are metallic if $n-m$ is integer multiple of 3 , otherwise semiconducting which means $1 / 3$ of SWNTs are metallic and 2/3 are semiconducting. Also armchair nanotubes $(n, n)$ are always metallic as the condition is always met, whereas the zigzag nanotubes are metallic only if $n$ is integer multiple of 3. The band gap of SWNT is equal to energy separation between the $\pi$ and $\pi^{*}$ orbitals of graphene band structure; at $\mathrm{K}$ point this gap is zero and it increases as one moves away in any direction [2, 5].

\subsubsection{Density of States for Semiconducting and Metallic SWNTs}

The electronic density of states (DOS) of system provides the information about the number of states per energy interval that are available for an electron to occupy and it depends on the dimensionality of the system. In one dimensional system, it is given by [2]

$$
g(E, 1 D)=\frac{d N}{d E}=\sqrt{\frac{2 \pi m^{*}}{h^{2}}} \sum_{i} \frac{1}{\sqrt{E-E_{\min , i}}}
$$

In the case of SWNT, the values $E_{m i n, i}$ correspond to minima of multiple 1D sub-bands. The band structure and electronic density of states of metallic and semiconducting nanotubes are shown in Fig. 2-5. The density of states at the Fermi level is zero for semiconducting nanotubes and finite for metallic single-walled nanotubes. The main features of the density of states for SWNTs are van Hove singularities which are manifested as peaks in optical absorption spectrum which will be discussed in section 2.1.5.
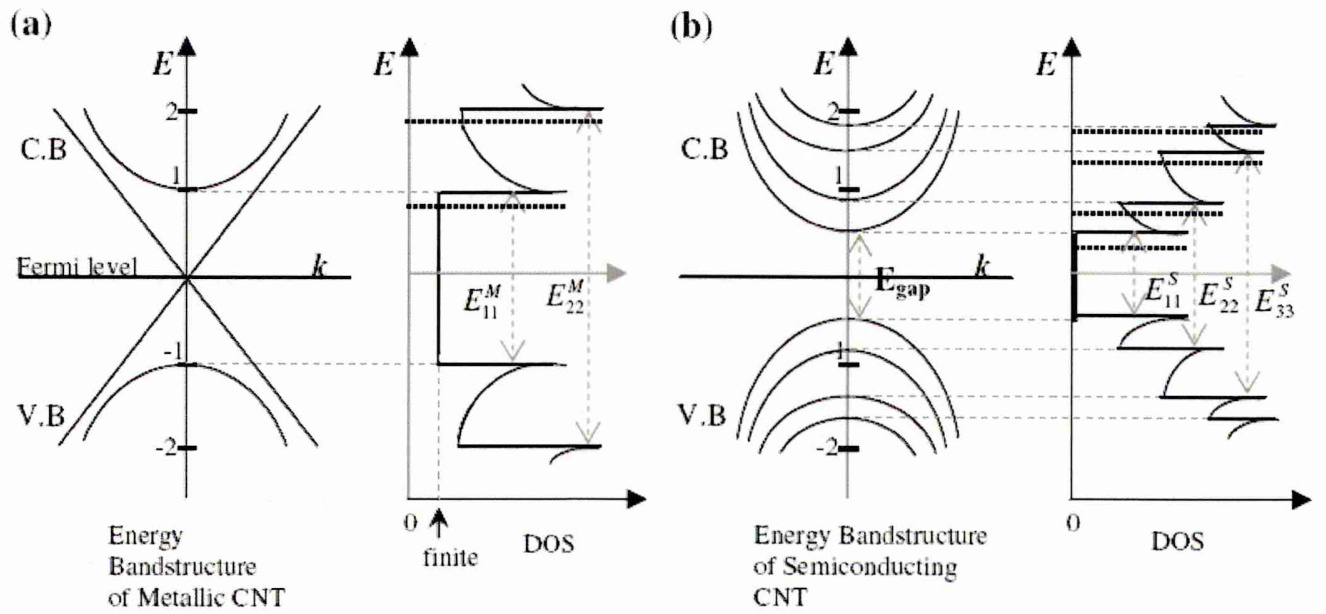

Figure 2-5: Band structure and electronic density of states (van Hove singularities) for (a) metallic and (b) semiconducting SWNTS. This figure is adapted from [7]. 


\subsubsection{Evidence of Excitons in Carbon Nanotubes}

Due to confinement of the electron and hole wave functions in two of the three spatial dimensions, there is strong coulombic interaction which leads to the formation of stable excitons with binding energies depending on the diameter, chirality and dielectric screening [8-10]. The excitons on carbon nanotubes support Wannier-Mott picture in spite of significant exciton binding energies of $0.4-1.0 \mathrm{eV}$ which is typically associated with Frenkel excitons $[11,12]$. This is due to large reported value of exciton size $(1-2 \mathrm{~nm})[11,13,14]$ which is much larger than lattice constant in carbon nanotubes and its wavefunction is delocalised over many atomic sites.

A schematic representation of excitonic states in carbon nanotubes is shown in Fig. 2-6. The left-hand picture shows the photogeneration of free electrons and holes in the second conduction-band (LUMO) and valence-band (HOMO) states respectively, followed by radiative recombination with emission of a photon at energy $E_{1}$. In the excitonic picture (right hand), individual electron and hole bands are not meaningful as the electrons and holes are strongly coupled. Therefore photoexcitation occurs directly into the excitonic state $E_{22}$, and the exciton relaxes to the $E_{11}$ excitonic state before radiative recombination. The excitonic states (labelled $1 \mathrm{u}$ in the diagram) are separated from the free-particle states by the exciton binding energy, and the curvature of the energy bands is given by the reduced exciton mass.
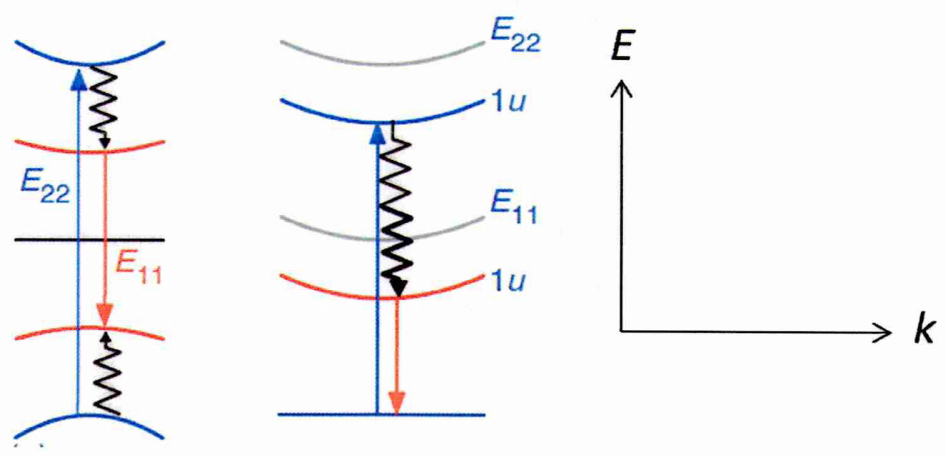

Figure 2-6: Schematic representation of single-particle states (left) and excitonic states (right) with excitation into $E_{22}$ and emission from $E_{11}$. This figure is taken from [15].

Electron density is confined to the plane of graphene sheet in carbon nanotubes and exciton wave functions are delocalised along the circumference of nanotube and extend over several nanometres along the nanotube axis [8]. Therefore variational model [16] was used 
initially to calculate binding energy of excitons because this model describes the motion of electron and hole under attractive coulombic interaction on a cylindrical surface with dielectric screening $\varepsilon$. Pedersen et al. [16] in 2003 used this model to describe the optical absorption spectra of SWNTs and calculated an exciton binding energy of $0.4 \mathrm{eV}$.

In 2004, Spataru et al. [9] calculated exciton binding energy of $\sim 1 \mathrm{eV}$ for semiconducting and $\sim 100 \mathrm{meV}$ for metallic carbon nanotubes using an ab-initio model and Zhao et al. [17] reported binding energies of $0.3-0.53 \mathrm{eV}$ for semiconducting SWNTs using a semiempirical approach. In short, a range of values of exciton binding energies $(400 \mathrm{meV}-1 \mathrm{eV})$ were reported for carbon nanotubes using different approaches $[9,16,17]$.

Experimentally, Wang et al. [11] and Maultzsch et al. [18] validated the excitonic character of optical excitation in carbon nanotubes using two photon luminescence excitation spectroscopy and reported excitonic states with different wave function symmetry, similar to $\mathrm{s}$ and $\mathrm{p}$ like states in a 3D hydrogen model as shown in Fig. 2-7. In this figure, $1 u$ is the lowest allowed excitonic state with odd $(u)$ symmetry and coupled with one photon absorption while $2 g$ is an "optically inactive" exciton state (dark exciton) with even $(g)$ symmetry that couples with two photon absorption. In two photon excitation, the initial and final states have same parity in contrast to single photon transitions in which initial and final states must have opposite symmetry. The strength of coulombic correlation (exciton binding strength) can be estimated from the energetic splitting of these one and two photon states.
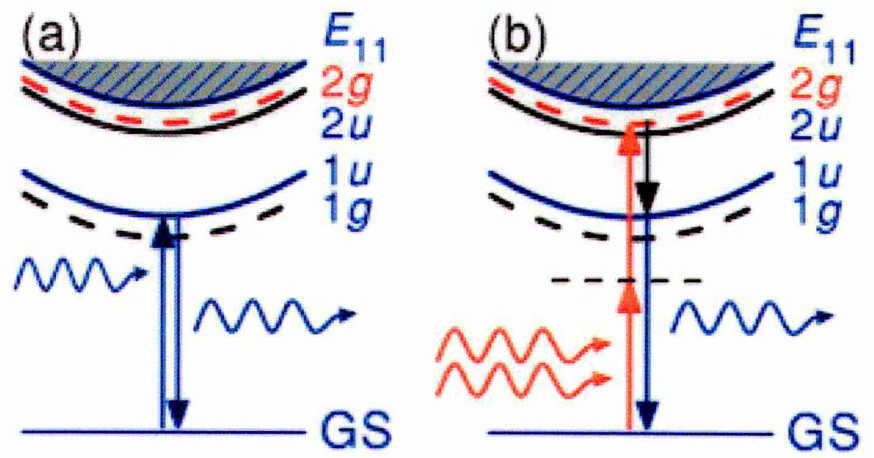

Figure 2-7: Schematic representation of one photon and two photon absorption in carbon nanotubes. (a) One photon absorption is linked to the excitonic states with odd (u) symmetry. (b) Two photon absorption couples with excitonic states with even ( $g$ ) symmetry. In both cases, fluorescence occurs from the lowest one photon active (1u) state. This figure is taken from [18]. 
In Fig. 2-8, the emission intensity following two photons excitation [11] is plotted as a function of excitation and emission energies. The emission energy is less than the excitation energy (by approximately $0.3 \mathrm{eV}$ ) which provides direct evidence of excitonic effect in SWNTs. No emission was observed from the nanotubes when the two photon excitation and emission energies were same (solid line in Fig. 2-8).

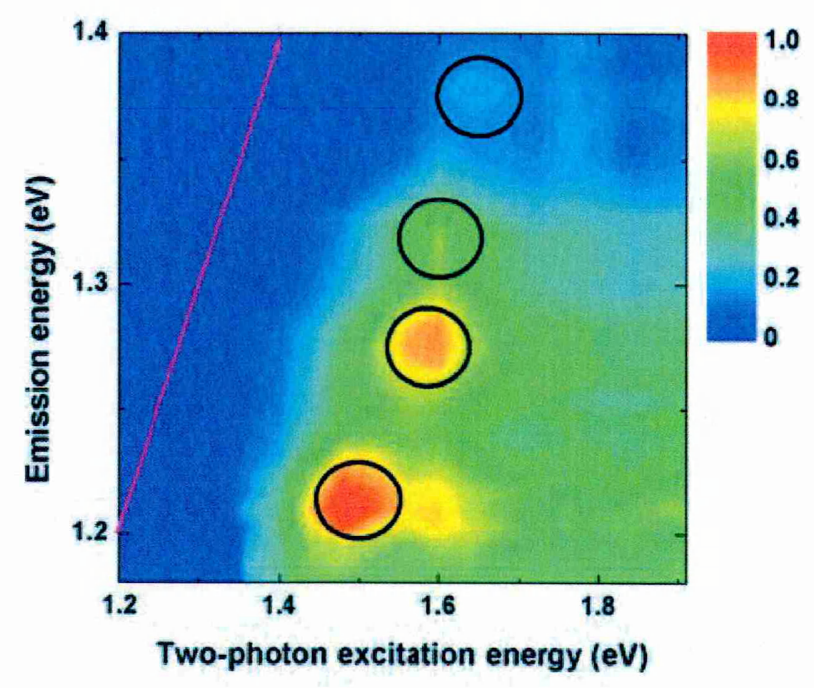

Figure 2-8: Contour plot of two photon excitation spectra of SWNTs taken from [11]. The one photon fluorescence emission is plotted as function of two photon excitation energy which clearly indicates the excitonic nature of SWNT optical transitions.

The exciton binding energy $E_{e x}$ can also be calculated from the diameter of carbon nanotube via following relation [19]

$$
E_{e x}=\frac{0.34 \mathrm{eV}}{d_{n}}
$$

In conclusion, an exciton binding energies of $0.3-0.42 \mathrm{eV}[11,18,20]$ was reported experimentally for different nanotubes with diameter $0.7-0.9 \mathrm{~nm}$. This large value of the exciton binding energy in carbon nanotubes indicates that excitons are much more stable at room temperature as compared to other semiconductor.

\subsubsection{Linear Optical Absorption of SWNTs}

The linear absorption spectrum of a suspension of HiPco SWNTs as a function of 
wavelength is shown in Fig. 2-9. The absorption spectrum of SWNTs is characterised by many sharp (narrow) peaks due to optical interband transitions in both semiconducting and metallic nanotubes i.e. due to optical transitions between the spikes in the density of states of SWNTs shown in Fig. 2-5. Each peak in the absorption spectrum corresponds to specific nanotube $(n, m)$ species.

The absorption spectrum in Fig. 2-9 is plotted from $400 \mathrm{~nm}$ to $1400 \mathrm{~nm}$ which covers the $E_{11}$ and $E_{22}$ transitions of semiconducting nanotubes as $E_{11}$ transition lies between 885 $\sim 1400 \mathrm{~nm}, E_{22}$ transitions lies between $885-\sim 475 \mathrm{~nm}$ and higher energy peaks are attributed to $E_{11}$ transition of metallic nanotubes for the HiPco nanotubes with diameter mostly centred between $0.7-1.2 \mathrm{~nm}$ [21].

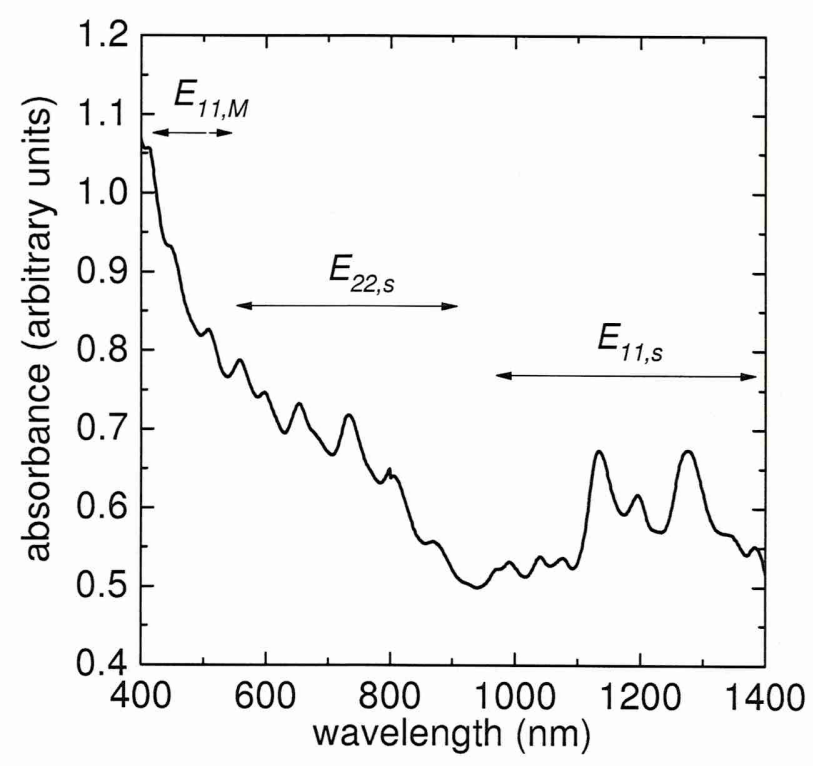

Figure 2-9: Linear absorption spectrum of a well isolated suspension of HiPco SWNTs, showing various sharp peaks with large background. The individual narrow peaks are due to interband transition for the specific $(n, m)$ species.

\section{Effect of Aggregation on Absorption Spectrum and Isolation of SWNTs}

Reich et al. [22] theoretically shown that van der Waals interactions between neighbouring SWNTs in bundle introduces extra dispersion into the band structure which causes broadening of peaks in the density of states. This broadening leads to broadening of features in the absorption spectrum and makes it difficult to resolve individual peaks. Following this, many attempts have been made to weaken the Van der Waals interaction between 
neighbouring nanotubes and to isolate the nanotubes from each other.

Bachilo et al. [23, 24] managed to isolate nanotubes in an aqueous suspension by surrounding them with sodium dodecyl sulfate (SDS) molecules. This allowed them to observe narrow fluorescence peaks and hence to identify the semiconducting species in their sample as shown in Fig. 2-10 (b). For these measurements, they excited the sample with photon energy equal to $E_{22}$ followed by nonradiative relaxation and fluorescence emission were obtained from $E_{11}$ as shown in Fig. 2-10 (a).

Isolation of nanotubes has also been achieved using other surfactants like sodium dodecyl benzene sulfonate (SDBS) [25], sodium cholate (SC) [26] or by using highly polar solvents such as NMP [27]. Another method which has been extensively used for the isolation of the nanotubes is by wrapping the nanotube with long-chain molecules such as single-strand DNA, RNA or cyclic peptides [28-31]. DNA wrapping has allowed separation of nanotubes with a single chirality [30]. All these methods are reviewed by Komatsu et al. [31].
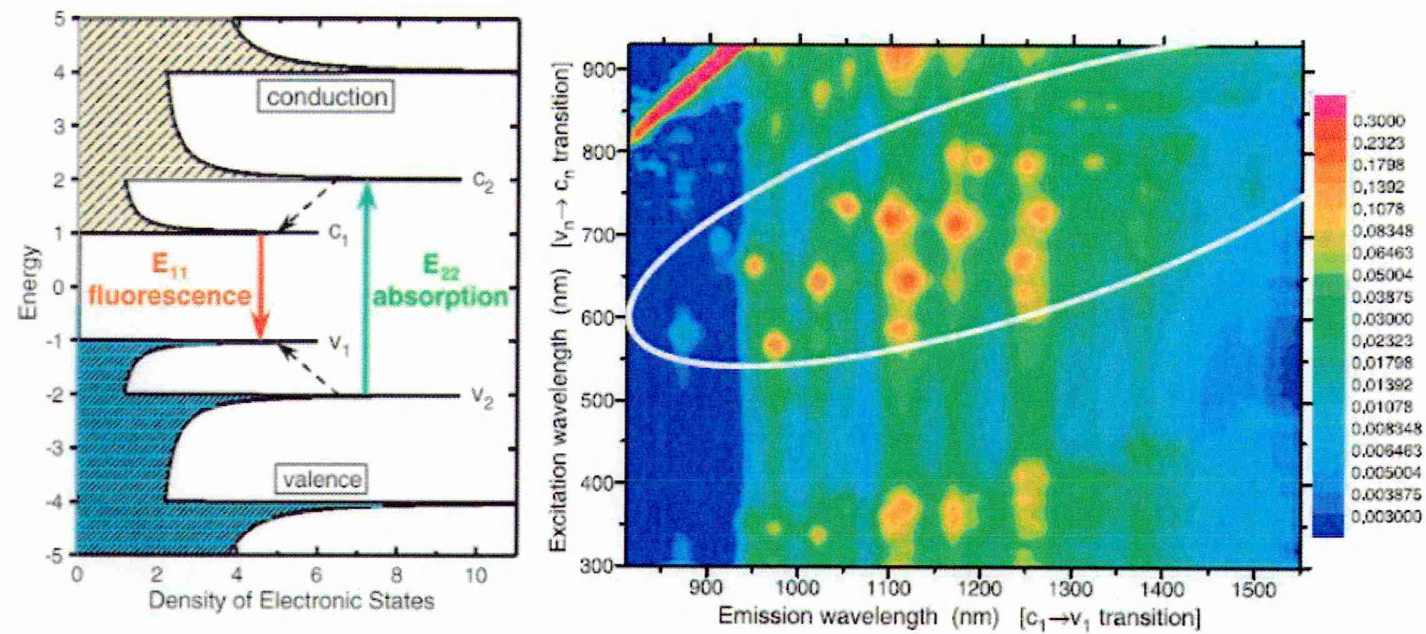

Figure 2-10: (a) Schematic of electronic density of states for semiconducting SWNTs showing the optical excitation of $E_{22}$ and fluorescence emission from $E_{11}$ (solid arrows). Dashed arrows in this figure denote the nonradiative relaxation before emission. (b) Contour plot of fluorescence of carbon nanotubes as function of excitation and emission wavelengths. This figure is taken from Bachilo [24].

\subsection{Diffusive Transport of Excitons in Carbon Nanotubes}

In the previous section, we reported the generation of strongly bound, uncharged excitons 
upon photoexcitation of small diameter semiconducting nanotubes. These excitons are delocalised along the nanotube circumference. In the direction of nanotube axis, they also display delocalised behaviour across several atomic sites (i.e. Wannier-like) and as a result, can move through the crystal lattice. However, these excitons are localised on the scale of typical mean free paths for scattering, and as a result can be viewed as quasi particles whose transport is affected by discrete scattering processes. The transport of excitons will be essentially one dimensional, and will be dominated by thermally-driven diffusion in the absence of large externally-applied fields.

\subsubsection{Normal Diffusion}

Diffusive (Brownian) motion can be visualised as a stochastic process defined on the points of lattice with equal spacing in which the particles undergo a random walk on the lattice. In 1905, Albert Einstein derived theoretical description of Brownian motion for a system with different dimensionality. In one dimensional system, it can be expressed as [32]

$$
\frac{\partial}{\partial t} n(x, t)=D \frac{\partial^{2}}{\partial x^{2}} n(x, t)
$$

where $D$ is diffusion coefficient and is measure of mobility of diffusing particles. Using initial condition $(t=0)$ i.e. $n(x, 0)=\delta(x)$, above equation can be easily solved to give

$$
n(x, t)=\frac{1}{\sqrt{4 \pi D t}} e^{-\frac{x^{2}}{4 D t}}
$$

The equation 2.9 describes the Gaussian distribution which gradually decreases in height and increases in width with time but its area is conserved as shown in Fig. 2-11. Hence in the case of normal diffusion (Brownian motion), random walks obey Gaussian statistics i.e. uniform distribution of intervals and the mean square displacement of the particles has a linear relationship with time i.e.

$$
\left\langle x^{2}(t)\right\rangle=2 D t
$$

Diffusive transport of the particles in one dimensional system is very important because through which we can explain the diffusion-limited reactions in quasi one dimensional 
system (SWNTs) and detail will be given in chapters 5 and 6 . The above relation can be modified for a system of dimension " $d$ "

$$
\left\langle r^{2}(t)\right\rangle=2(d) D t
$$

The important property of regular diffusion is its universal time dependence regardless of dimension as given in equation 2.10.

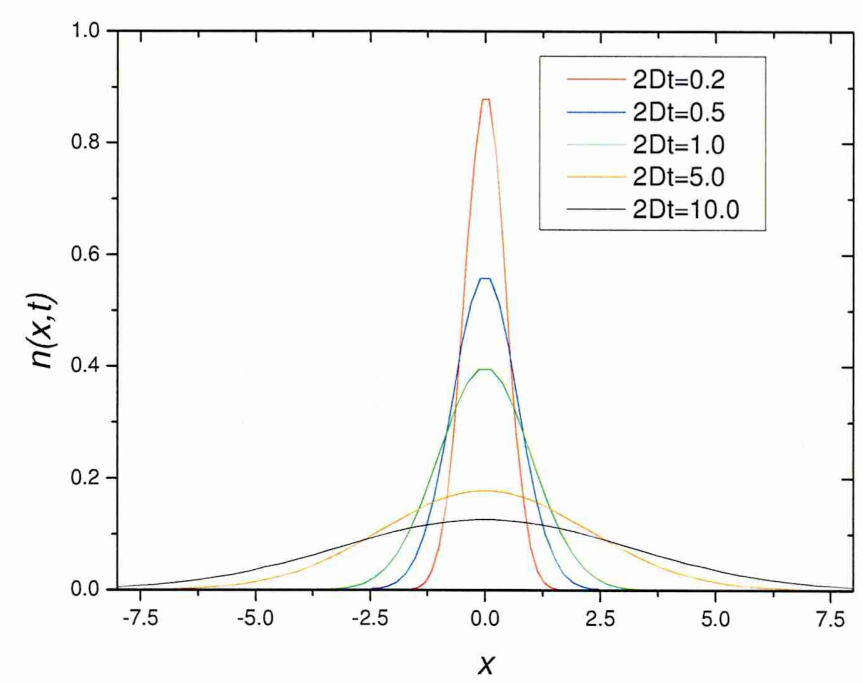

Figure 2-11: Diffusive growth of 1D Gaussian distribution with time.

\subsubsection{Anomalous Diffusion}

As we discussed above, the random walk of the particles normally obeys Gaussian statistics at long times and their average mean square displacement has a linear relationship with time. However in many physical systems, diffusion exhibits anomalous behaviour and its mean square displacement has non linear dependence on time. The diffusion relation for anomalous diffusion is modified as [33]

$$
\left\langle x^{2}\right\rangle=\frac{2 k_{\gamma}}{\Gamma(1+\gamma)} t^{\gamma}=2 D_{\gamma} t^{\gamma} .
$$

where $D_{\gamma}=\frac{k_{\gamma}}{\Gamma(1+\gamma)}$ is anomalous diffusion coefficient. Hence for $\gamma=1$ and $k_{l}=D$, above relation (eq. 2.11) reduces to the normal diffusion relation.

For $\gamma>1$, diffusion is enhanced and mean square displacement scales faster than linearly 
with time. This type of anomalous diffusion is known as superdiffusion, for example $\gamma=2$ corresponds to ballistic transport of particles [34].

For $0<\gamma<1$, the anomalous transport is described as subdiffusion in which diffusion is slower than normal diffusion. Sub-diffusive random walks can arise from different mechanisms, including:

(i) Continuous Time Random Walks (CTRW), in which the distribution of displacements and waiting times is non-Gaussian and may include the possibility of very long waiting times (trapped particles) or very long flights (Levy flights).

(ii) Fraction Brownian motion (FBM) (also referred to as long range correlated walks), in which successive jumps are interdependent.

(iii) Obstructed diffusion in a disordered material, where normal diffusion is constrained by randomly-distributed obstacles including the barriers, potential wells, particle crowding etc [33, 35-38]. In one dimensional system, there is little difference between potential barriers and wells. This distinction becomes very important in higher dimensions, where barriers can be avoided by walking around them. This obstructed diffusion in disordered system with randomly-distributed obstacles will be basis of our Monte Carlo model of anomalous diffusion in chapter 6.

These mechanisms give rise to subdiffusion but have different properties [32]. In this report of diffusion-limited exciton-exciton interactions on carbon nanotubes, we will encounter situations where either normal or anomalous diffusion occur, depending on the density of defects present and will be discussed in detail in chapter 6.

\subsubsection{Diffusion in Carbon Nanotubes}

Exciton transport in SWNTs is characterized by the diffusion coefficient $D$. The range of values of the diffusion coefficient were reported in SWNTs that varies significantly from $0.1 \mathrm{~cm}^{2} / \mathrm{s}$ to $120 \mathrm{~cm}^{2} / \mathrm{s}$ and is unclear whether these different values of the diffusion coefficient are due to extrinsic properties of SWNTs or may be due to measurement techniques. For comparison purpose, these results are grouped into three different categories. 


\section{Photoluminescence (PL) Quenching}

Photoluminescence (PL) of SWNTs is suppressed in the presence of defects including sidewall defects and defects at the ends of nanotubes. A wide range of PL quantum yield for SWNTs is reported that varies from $0.1 \%$ to $30 \%$ [3, 39-43] depending on sample preparation and surfactants used. Here we summarised few of them

Rajan et al. [44] fitted the Heller et al. [45] length dependent PL quantum yield data of highly sonicated HiPco nanotubes in SC. They considered tube ends as exciton quenching sites and proposed a simple 1D diffusion model to understand the length dependent PL quantum yield. They obtained $D=6 \mathrm{~cm}^{2} / \mathrm{s}$ by assuming $1 \mathrm{~ns}$ radiative lifetime of excitons. Cognet et al. [46] obtained diffusion constant of $D=0.4 \mathrm{~cm}^{2} / \mathrm{s}$ through fluorescence quenching of HiPco SWNTs in SDBS. In their case, quenching sites was created through the chemical reaction of single molecule with SWNTs. Yoshikawa et al. [47] studied the exciton transport in suspended SWNTs by PL imaging spectroscopy. They used oxygen molecules to create PL quenching sites in SWNTs and reported the value of $D=2 \mathrm{~cm}^{2} / \mathrm{s}$.

Hertel et al. [40] considered tube ends and sidewall defects as quenching sites and determined $D=(10 \pm 0.4) \mathrm{cm}^{2} / \mathrm{s}$ from analysis of time-resolved PL decay and length dependent PL quantum yield for CoMoCat SWNTs in SC. Liu et al. [48] reanalyzed the Hertel et al. [40] PL quantum yield data of defective SWNTs (with defect density 0.0039 / $\mathrm{nm}$ ) to get a diffusion coefficient of $D=2.2 \mathrm{~cm}^{2} / \mathrm{s}$. In contrast to previous studies, Miyauchi et al. [49] reported that there exists an upper limit of PL lifetimes for SWNTs due to some other dominant non-radiative relaxation paths (such as quenching at defect sites or scattering by doped carriers) than quenching at the ends of nanotube. They determined diffusion constant of $D=1 \mathrm{~cm}^{2} / \mathrm{s}$ from the length dependent PL lifetime in DNA wrapped HiPco SWNTs.

\section{Photoluminescence (PL) Saturation}

At high excitation fluences, the quantum yield of SWNTs is limited by exciton-exciton annihilation (Auger recombination) which is efficient process for removing excitons nonradiatively and will be discussed in detail in section 2.4. Wang et al. [3] used this process to explain early fast decay in their time resolved fluorescence data and reported $D=90 \mathrm{~cm}^{2} / \mathrm{s}$ for HiPco SWNTs in poly (acrylic acid) (PAA). Murakami et al. [50] proposed diffusion- 
limited exciton-exciton annihilation model to explain the PL saturation and determined diffusion coefficients of $D=0.42 \mathrm{~cm}^{2} / \mathrm{s}$ in CoMoCat SWNTs in $\mathrm{D}_{2} \mathrm{O}$ with SC. A diffusion coefficient of $D=44 \mathrm{~cm}^{2} / \mathrm{s}$ [51] is reported for air suspended SWNTs and its value decreases with excitation power due to exciton-exciton annihilation effects.

\section{Pump Probe Spectroscopy}

Pump probe spectroscopy was also used to determine the transport of excitons in nanotubes. Korovyanko et al. [52] reported $D=120 \mathrm{~cm}^{2} / \mathrm{s}$ for HiPco nanotubes in deionised water $\left(\mathrm{D}_{2} \mathrm{O}\right)$ with anionic surfactant lithium dodecyl sulfate. Harrah et al. [53] reported $D=4 \mathrm{~cm}^{2} / \mathrm{s}$ for $(6,5)$ nanotubes in SC. Lüer et al. [13] used diffusion-limited exciton-exciton annihilation to obtain diffusion coefficient of $D=0.1 \mathrm{~cm}^{2} / \mathrm{s}$ for CoMoCat $(6,5)$ nanotubes in SC.

In conclusion, many attempts have been made to evaluate the diffusion coefficient in carbon nanotubes using variety of techniques and samples. However, determination of the exact value of the diffusion coefficient experimentally remains a challenge. Hence a more complete investigation is required to clearly demonstrate the diffusive transport of exciton in SWNTs and we will address this in chapter 5 of this thesis.

\subsection{Reaction Kinetics: Reaction-limited and Diffusion-limited Processes}

Reaction-diffusion systems are an important class of many body, stochastic nonequilibrium systems in which constituent particles move due to thermal diffusion and interact in a specific manner when they are in contact with each other. We are interested in reaction kinetics i.e. time dependence of concentration of the reactants which is strongly influenced by the transport of reactants. The reaction kinetic is governed by two characteristic time scales: reaction time $t_{R}$ and diffusion time $t_{D}$, depending on whether overall reaction rate is limited by the transport of particles or their reaction probability once they are in contact. The diffusion time $t_{D}$ is the time taken by the reacting particles to meet each other and $t_{R}$ is the time taken by the reactants to react once they are in contact i.e. within the reaction range of each other.

When $t_{D} \ll t_{R}$, the process is limited by reaction time as diffusion is very fast and is 
known as reaction-limited process. This process can be described by the classical rate equation and depends on the global concentration. In the case of $t_{D} \gg t_{R}$, the process is controlled by the diffusive transport of reactants and is called the diffusion-limited process. In this limit, reaction is immediate when particles are within in the reaction range and kinetics is largely influenced by local fluctuation in the concentration of the reactants and cannot be describe by simple classical rate equations [32].

\subsubsection{The 1D Single-species Bimolecular Annihilation and Coagulation Reaction}

Perhaps the simplest examples of diffusion-limited reactions are irreversible single species bimolecular annihilation and coalescence (also referred to as coagulation or fusion). The annihilation process is described by $A+A \rightarrow 0$, where both particles diffuse with same diffusion constant $D$ and annihilation is immediate upon encounter. The coalescence process described by

$$
A+A \rightarrow A
$$

occurs when reacting particles combine together to form a single particle or when one of them is annihilated or converted into inert species etc. This is one of only a few exactlysolvable stochastic processes, and is perhaps the simplest system which exhibits a rich variety of kinetics behaviours, including self-organised criticality and dynamical crossover between the reaction-limited and diffusion-limited regime with different reaction dynamics [54].

The rate equations and long time asymptotes of these different reaction dynamics can be found using a simple scaling argument, in terms of the dimensionality $d$, and exponent $\gamma$ of the random walk [55-57] where the root mean square displacement of particle is given by $z(t) \sim t^{\gamma / 2}$, where $\gamma=1$ for normal diffusion.

If the diffusion is modelled as series of random hops at interval $\tau$ on lattice with lattice spacing $\Delta z$, a normal (Fickian) diffusion with $\gamma=1$, then the volume swept out by a particle is $z^{d}$ which contains $(z / \Delta z)^{d}=(t / \tau)^{d / 2}$ distinct lattice sites. The number of hops made is $(t / \tau)$. Hence for $d>d_{c}$, where $d_{c}=2$ is the upper critical dimension, then at long times only a small fraction of possible sites are visited. The reaction rate depends on the local concentration (availability of a pair of particles for reaction) and can be explained by the classical rate equation with a time-independent rate constant (the "law of mass action") 


$$
\frac{d n}{d t}=-k_{r} n^{2}
$$

Here, $k_{r}$ is the rate constant related to reaction probability per encounter and $n$ is the concentration. The above equation is easily integrated to give

$$
n(t)=\frac{n_{0}}{1+n_{0} k_{r} t}
$$

Here $n_{0}$ is the initial concentration of reactants. The rate of reaction depends on the local concentration of reactants and reaction rate $k_{r}$. In this reaction-limited or "mean-field' regime, the system is locally homogenous or "well-mixed". At sufficiently long time, the particle concentration follows $t^{-1}$ decay behaviour and is independent of dimensionality $d$ and the diffusion exponent $\gamma$.

For $d \leq d_{c}$, the situation is different and cannot be explained by a simple classical rate equation. In this case the diffusion is compact i.e. each possible site is visited multiple times and a particle reacts with one of particles in its immediate neighbourhood [58].The average distance between the neighbouring particles is $x \sim 1 / n^{1 / d}$ and time taken by a particle to diffuse an interparticle distance $x$ is $\tau \sim\left(\overline{x^{2}}\right)$ which is equivalent to the total time required for the reaction because reaction is immediate upon encounter. Hence the rate of change of the concentration in the time interval $\Delta t$ is [32]

$$
\frac{d n}{d t}=\frac{\Delta n}{\Delta t} \sim-n^{1+2 / d}
$$

The reaction order exceeds the classical mean field value of 2 and the reaction kinetics is anomalously slow compared to the mean-field case. For $d=1$ the rate equation 2.14 becomes [54]

$$
\frac{d n}{d t}=-k_{d} n^{3}
$$

where $k_{d}$ is diffusion rate. The reaction kinetics in this diffusion-limited regime is determined by the transport of reactants and local fluctuation in the concentration of reactants. One can get the information about the decay of concentration through the integration of rate equation 2.15 


$$
n(t)=\frac{n_{0}}{\sqrt{1+2 k_{d} n_{0}^{2} t}}
$$

Asymptotically the concentration of reactants follows $n \propto t^{-1 / 2}$, a well known anomalous 1D reaction rate. The equation 2.16 is only true in the asymptotic limit. At short times, the reaction rate will depend on the distribution of particle separations and hence on the initial spatial distribution.

Note that in the case of particle crowding, binding or obstacles, the effective reaction order of rate equation 2.14 is increased (exceeds the value of 3 ) i.e. $\frac{d n}{d t}=-n^{1+2 / \gamma d}$, where $0<\gamma<1$ and kinetics become even slower (both the diffusion and the reaction rates are anomalous). This will be discussed in detail in chapter 6 .

The above dimensional arguments for $A+A$ reactions are sufficient to determine both upper critical dimensions and decay exponents for reaction and diffusion-limited regimes. However exact solutions are required to determine the amplitude $k_{d}$. Ben-Avaraham [59] derived an exact solution for diffusion-limited coalescence using the method of empty intervals (also known as interparticle distribution function) which yield an asymptotic decay of

$$
n(t) \sim \frac{1}{\sqrt{2 \pi D t}}
$$

where $k_{d}=\pi D$. The equation 2.17 shows that exponent and amplitude are universal i.e. independent of reaction rate (microscopic detail of the system) and initial population. This is an example of "universal" behaviour. Together with the power-law decay which shows a scale-invariant behaviour. It indicates that the asymptotic diffusion-limited regime arises from self-organised criticality.

Experimental evidence for anomalous reaction rate and fluctuation effects in 1D system was reported from several studies of exciton fusion in polymers [60, 61] and ultrathin molecular wires [62], where a population decaying approximately as $t^{-1 / 2}$ was observed at intermediate timescales over wide range of excitation densities.

In conclusion, the decay rate of the reacting particles at long time depends on the dimensionality of the system but it is independent of initial concentration of particles. For $d>2$, the mean field theory of classical rate equation applies and is reaction-limited process, 
whereas for $d \leq 2$, the diffusion is important and cannot be explained by the classical rate equations. Hence $d=2$ is upper critical limit at which transition between the reactionlimited and diffusion-limited regimes occurs [32].

\subsubsection{Finite System Size}

If the size of the system is finite, then after a finite time all particles will have interacted and coalesced, leaving a single remaining particle. From equation 2.17 , a population of 1 particle in a system of size $L$ is achieved when $t=\frac{L^{2}}{2 \pi D}$. However, when the particle number is small, the system cannot be well described by an average concentration and hence equation 2.17 will fail. The expected behaviour of a finite system is an exponential decay in which the system size determines the decay time. Hence it will lead to another dynamical crossover from the universal power law of an infinite system to exponential decay of a finite system. Simon [63] studied bimolecular coagulation (coalescence) and annihilation on 1D wire of finite length $\mathrm{L}$ where he mapped the dynamics onto the Schrödinger equation and gives following expression for the average number of massless fermions diffusing on 1D lattice

$$
\begin{aligned}
n\left(t, c_{0}, N_{s}, L\right) . L & =\frac{1-\left[1-(2-f) c_{0}\right]^{N_{s}}}{(2-f)}-\frac{1}{(2-f)} \\
& \times \sum_{j=1}^{N_{s}-1}\left\{\frac{1-(-1)^{j}\left[1-(2-f) c_{0}\right]^{N_{s}}}{\frac{1+\left[1-(2-f) c_{0}\right]^{2}}{2\left[1-(2-f) c_{0}\right]}-\cos \left(\frac{\pi j}{N_{s}}\right)}-\frac{1-(-1)^{j}\left[1-(2-f) c_{0}\right]^{N_{s}}}{1-\cos \left(\frac{\pi j}{N_{s}}\right)}\right\} \\
& \times \sin ^{2}\left(\frac{\pi j}{N_{s}}\right) \exp \left[-4 D\left(\frac{N_{s}}{L}\right)^{2} t\left(1-\cos \left(\frac{\pi j}{N_{s}}\right)\right)\right]
\end{aligned}
$$

We have written this equation in terms of dimensioned variables rather than the dimensionless time and length used by Simon [63]. $N_{s}=\frac{L}{\Delta Z}$ is number of lattice sites, $j$ is an index referring to lattice sites, $c_{0}$ is the initial concentration per lattice sites, and $f=1$ for pure coagulation and 0 for pure annihilation. Hence for pure coagulation $(f=1)$ and an initially full lattice $\left(c_{0}=1\right)$, the above equation simplifies to

$$
n\left(t, N_{s}, L\right) . L=1+\sum_{j=1}^{N_{s}-1} \frac{\sin ^{2}\left(\frac{\pi j}{N_{s}}\right)}{1-\cos \left(\frac{\pi j}{N_{s}}\right)} \times \exp \left[-4 D\left(\frac{N_{s}}{L}\right)^{2} t\left(1-\cos \left(\frac{\pi j}{N_{S}}\right)\right)\right]
$$


At long times, small $j$ terms dominate and we obtain

$$
n(t, L) . L-1=2 \sum_{j=1}^{L / \Delta z-1} \exp \left[-2\left(\frac{\pi}{L} j\right)^{2} D t\right]
$$

At intermediate times, it reproduces a $n \sim t^{-1 / 2}$ power law but at long times it decays with a single exponential with a monoexponential time constant of $\frac{(L / \pi)^{2}}{2 D}$ which is of the order of time taken for two particles at either end of the nanotubes to diffuse into middle and meet.

\subsubsection{The 1D Reaction-Diffusion System with Both Finite Reaction and Diffusion Rate}

The discussion in the previous section 2.3.1 assumed that particles which meet always react i.e. the reaction probability per attempt $(p)$ is unity. Hence for $d<d_{c}$, the reaction kinetics were found to be always anomalous. In reality there is no reason for this to be the case and we must consider the effect of a finite reaction probability, where the both reaction and diffusion will influence the rate of population decay. Unfortunately the exact solutions available for the bimolecular coalescence problem are no longer valid for a finite reaction probability.

Hoyuelos and Martin (H\&M) [64, 65] studied bimolecular coagulation reaction in $1 \mathrm{D}$ system with diffusion and reaction probability $(p)$, and determined the population decay $\rho(t)$ using an analytical scaling theory as

$$
\rho(\tau)=\frac{\rho_{o}}{2} \frac{1+\sqrt{\left(1+\frac{\rho_{c}}{\rho_{o}}\right)^{2}+\frac{4 p^{2}}{\pi} \tau}}{1+\frac{\rho_{c}}{2 \rho_{o}}+\rho_{o} p \tau} .
$$

where $\rho_{o}$ is the initial population, $\tau$ is dimensionless time and is defined as $\tau=t / t_{\text {hop }}$ where $t_{\text {hop }}=(\Delta z)^{2} / 2 D, \Delta z$ is a lattice spacing. $\rho_{c}$ is crossover density which describes particle concentration below which the asymptotic $t^{-1 / 2}$ behaviour is observed, and this $\rho_{c}$ becomes smaller if $p$ is decreased because the distribution of the particles has more chance to 'self-stir' and randomise. It was also found that $p$ is only important at short times. At long time, population decay follows a $t^{-1 / 2}$ behaviour for all the values of $p$. 
On other hand, Zhong and Ben-Avraham (ZBA) [54] derived a very similar relation for the same $1 \mathrm{D}$ reaction-coalescence problem with both finite reaction and diffusion rates, using a completely different theoretical approach, based on the inter particle distribution function (IPDF) [55], which represents the mean distance between particles at given density. This was an extension of their previous work $[55,66]$ in which they considered infinite reaction rate $(p=1)$ i.e. population decay is always diffusion-limited. Their relation can be expressed as

$$
\rho(\tau)=\frac{\rho_{o}}{2} \frac{(1-p)+\sqrt{\left(1-p+\frac{\rho_{c}}{\rho_{o}}\right)^{2}+\frac{4 p^{2}}{\pi} \tau}}{(1-p)+\frac{\rho_{c}}{2 \rho_{o}}+\rho_{o} p \tau}
$$

where the parameters are defined identically to those for the H\&M equation 2.19. The small difference between the H\&M and ZBA equations will be discussed in chapter 5 .

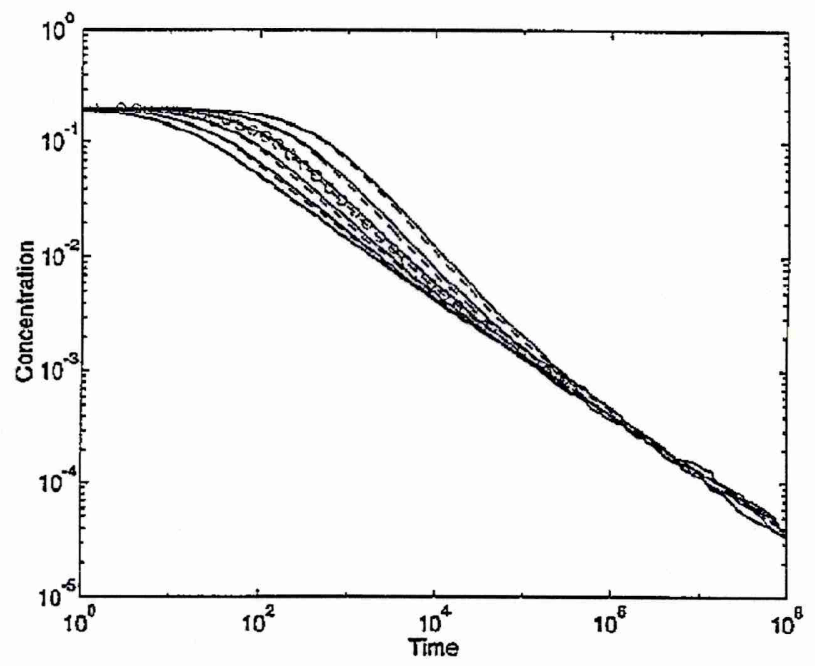

Figure 2-12: Theoretical prediction of population decay as a function of time, taken from [54]. The detailed description is presented in the text.

The equation 2.20 is plotted in Fig. 2-12 for different values of $p$, showing clearly that at long times, all traces converge to a $t^{-1 / 2}$ power law decay which is diffusion-limited annihilation in $1 \mathrm{D}$ system. This shows that at long times, diffusion dominates it leads to a $t^{-1 / 2}$ power law decay. At early time and lowest reaction probability, the reaction-limited process becomes important and the traces tend to $t^{-1}$ power law decay behaviour as shown in Fig. 2-12. Hence at early times, reaction obeys classical kinetics because of the ability of 
particles to pass through one another without reacting. Therefore, they self-stir and randomise. However, this randomisation becomes less important with increasing $p$ and the deviation from $t^{-1 / 2}$ behaviour at early times becomes smaller as shown in Fig. 2-12.

\subsection{Exciton-exciton Annihilation: Reaction-limited and Diffusion-limited Annihilation in Carbon Nanotubes}

The decay dynamics of excitons in SWNTs is governed by their interaction with phonons, defects, and impurities as well as with other excitons. As a result of these interactions, different dynamical competing processes occur including trapping of excitons at defects and exciton-exciton annihilation. When intensity of excitation is high enough to create two or more excitons per nanotube, then exciton-exciton annihilation $[3,67]$ process comes into play. In this process, one exciton $(\mathrm{X})$ relaxes rapidly to the ground state by giving its energy to the other exciton $(X)$, which is then promoted to a higher energy excited state $\left(X^{*}\right)$ [56]. This excited state rapidly loses energy through interactions with the lattice, returning to the unexcited exciton state $(\mathrm{X})$ :

$$
X+X \rightarrow X^{*} \rightarrow X+\text { heat }
$$

It is therefore postulated that this reaction will follow similar kinetics to the prototypical bimolecular coalescence reaction $\mathrm{A}+\mathrm{A} \rightarrow \mathrm{A}$ described above. Note that although frequently termed exciton annihilation in the carbon nanotube community, this process is a coalescence rather than annihilation reaction which would require both excitons to disappear.

Exciton-exciton interaction is a dipole-dipole interaction as compared to Auger recombination of free carriers in which carriers exchange energy through the Coulomb interaction. In Auger recombination of free carriers, an electron and hole recombine and excess energy is given to another particle which is promoted to higher energy levels; i.e. three particles are involved different from exciton-exciton annihilation which is a two body processes.

\subsubsection{Exciton-exciton Annihilation in SWNTs Neglecting Transport Effects}

Many theoretical and experimental papers have been published in which exciton-exciton annihilation is reported as the decay mechanism without considering the exciton transport. 
They consider the excitons to be delocalised along the length of the nanotubes. We summarise the main papers and results in this section.

Ma et al. [67] first reported this exciton-exciton annihilation process in semiconducting carbon nanotubes. The authors ruled out diffusion-limited annihilation on the basis of the results shown in Fig. 2-13 in which they used five different excitation intensities to excite the nanotubes.
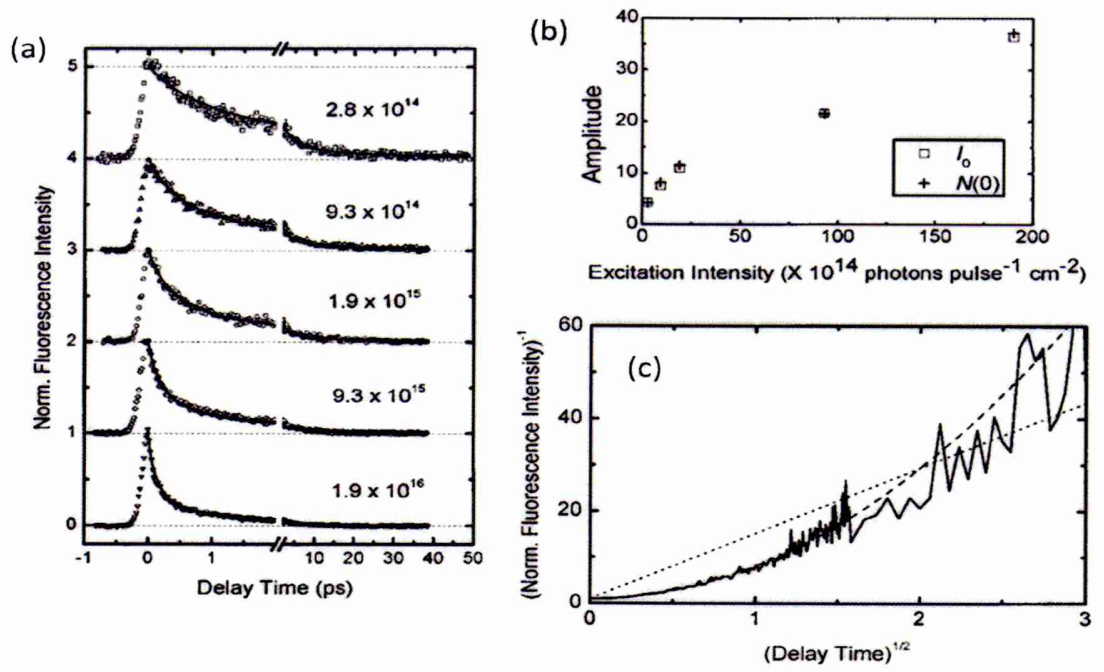

Figure 2-13: (a) Normalised time-resolved fluorescence intensity Vs time delay at different excitation intensities. (b) Maximum fluorescence intensity $I_{0}$ and fitted parameter $N(0)$ Vs excitation intensity. (c) Inverse of the normalised fluorescence intensity $\left(I(t) / I_{0}\right)$ plotted against $t^{1 / 2}$ for the highest excitation intensity. The dashed line is obtained by assuming $d \geq 2$ and dotted line for one dimension exciton diffusion. This figure is taken from [67].

They state that strong dependence of early fluorescence decay on excitation intensity (Fig. 2-13 (a)) and non-linear dependence of maximum fluorescence amplitude on the excitation intensity (Fig. 2-13(b)) suggest the exciton-exciton annihilation is the process responsible for the reduction of excitons. They used a classical rate equation 2.12 to fit their experimental data as shown in Fig. 2-13 (a) by assuming that dimensionality of exciton diffusion is $d \geq 2$. They made conclusion on the basis of result shown in Fig. 2-13 (c), where inverse of normalised fluorescence intensity is plotted versus $\sqrt{t}$ for highest excitation intensity. This figure shows that early time decay does not fit to $t^{-1 / 2}$, but is slightly better fitted by a $t^{-1}$ decay.

We will return to this point when we will discuss our own results in chapter 5 , but note for 
now that: (1) the $t^{-1 / 2}$ decay occurs at asymptotically long times. However, it is not clear whether the results of $\mathrm{Ma}$ et al. [67] fall into the asymptotic region as these results are presented only upto a timescales of few picoseconds; (2) although Ma et al. [67] have asserted that a $t^{-1}$ behaviour is incompatible with one-dimensional behaviour, this assertion is only true when the reaction rate is infinite $(p=1)$ as discussed in previous section.
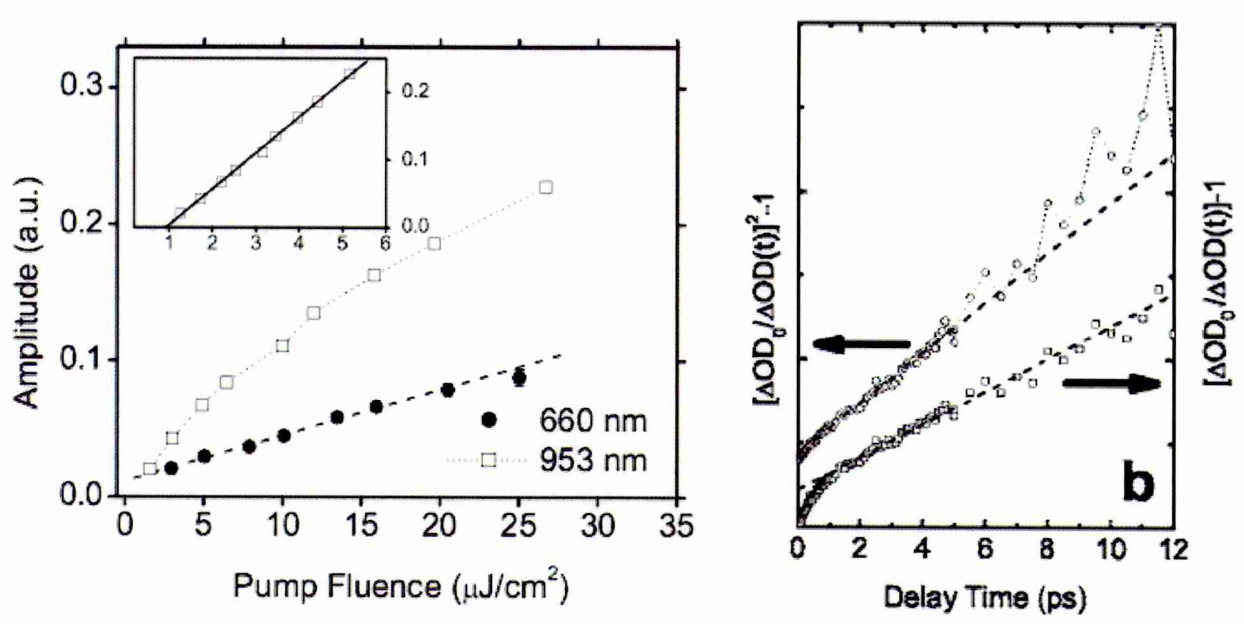

Figure 2-14: (a) Max. Amplitude of transient absorption kinetics probed at $953 \mathrm{~nm}$ (open squares) and $660 \mathrm{~nm}$ (filled circle) as a function of pump fluence. Inset show same data measured at $953 \mathrm{~nm}$ but plotted on the scale of square root of pump intensity. This figure is taken from Ma [68]. (b) Pump probe data taken from Valkunas [69].

The same group Ma et al. [68] used frequency resolved transient absorption spectroscopy to examine the same process, as shown in Fig. 2-14 (a). They excited $E_{11}$ state but probed both the $E_{11}$ and $E_{22}$ states of $(8,3)$ nanotubes and observed a linear dependence of amplitude of transient absorption signal on pump intensity when probed at $660 \mathrm{~nm}$ ( $E_{22}$ transition), but saturation in the case of probing at $953 \mathrm{~nm}\left(E_{11}\right.$ transition of $(8,3)$ nanotube). They interpreted this strong correlation between kinetics observed at $953 \mathrm{~nm}$ and $660 \mathrm{~nm}$ as reaction-limited exciton-exciton annihilation. Their rejection of a contribution of exciton transport to the reaction kinetics led them to conclude that the excitons must be substantially delocalised along the nanotubes.

The above study was extended by Valkunas et al. [69], but the same conclusion reached. Again, as shown in Fig. 2-14 (b), they report that the early dynamics are closer to $t^{-1}$ than $t^{-1 / 2}$, and from this they conclude (erroneously) that $1 \mathrm{D}$ diffusion plays no role. They evoke a complex manifold of multi-exciton states to describe the slow decay at long timescales. 
Huang et al. [70] in 2006 claimed experimental evidence of transitions from $3 \rightarrow 2 \rightarrow 1$ excitons per SWNT using a quantised Auger recombination model of excitons. They extracted time constants of $3.2 \mathrm{ps}$ and $1.9 \mathrm{ps}$ for the two and three e-h pair states by fitting the experimental data using a multiple exponential function as shown in Fig. 2-15

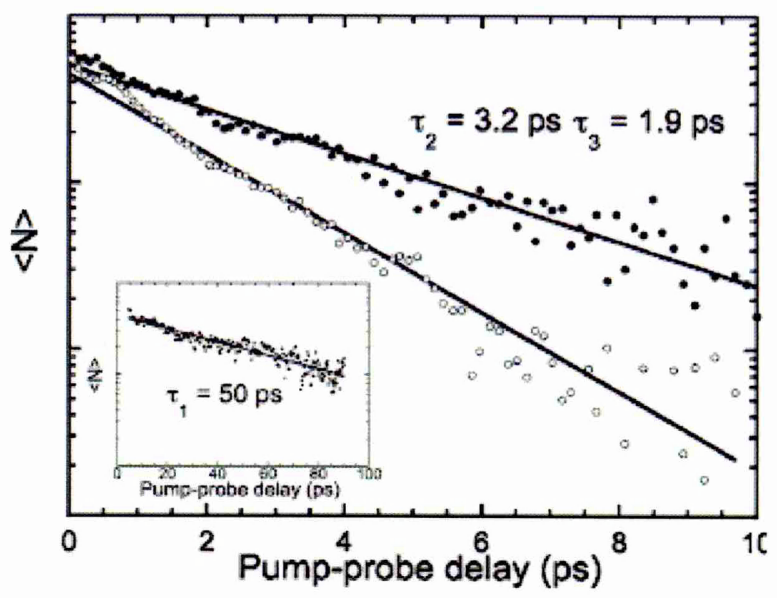

Figure 2-15: One (inset), two (filled circle) and three (open circle) e-h pairs dynamics extracted from experimental data of Huang [70].

They neglect diffusion on the grounds that "the SWNTs are short enough (mean length $\sim 380 \mathrm{~nm}$ ) such that two excitons are not required to diffuse before recombining (mean free path $\sim 1000 \mathrm{~nm}$ )". However, considering the fact that the mean free path for electron-phonon scattering is estimated to be a few $\mathrm{nm}$ at most [41] makes such as this statement hard to justify. For typically quoted diffusion coefficients and radiative recombination that occurs at a time scale of the order of $10 \mathrm{~ns}$ [41] implies a diffusion length of the order of $1000 \mathrm{~nm}$. For the highest reported values of diffusion coefficient, the mean time taken for a population of two excitons to meet on a nanotube of length $380 \mathrm{~nm}$ is $\sim 3 \mathrm{ps}$. Hence the time for diffusion cannot be neglected compared to the reported time constants, in contrast to the claim by Huang et al. [70].

\subsubsection{Diffusion-limited Exciton-exciton Annihilation}

In the limit of low exciton density, the excitons are far apart from each other and take some time to diffuse along the nanotubes in order to meet each other. So in this regime, exciton-exciton annihilation is limited by the diffusive transport of excitons and depends on 
diffusion time instead of reaction time.

Russo et al. [71] observed that the decay of the exciton population in $\operatorname{HiPco}(8,6)$ and $(7,6)$ nanotubes showed a power law $1 / \sqrt{t}$ dependence up to $\sim 1 \mathrm{~ns}$ which they attributed to diffusion-limited exciton annihilation in a 1D system. Their results are shown in Fig. 2-16 in which they used different functions (power law to multiple exponential) to fit their experimental data. From Fig. 2-16, it is clearly seen that $t^{-1 / 2}$ fit is more accurate than mono, bi and tri-exponential functions.
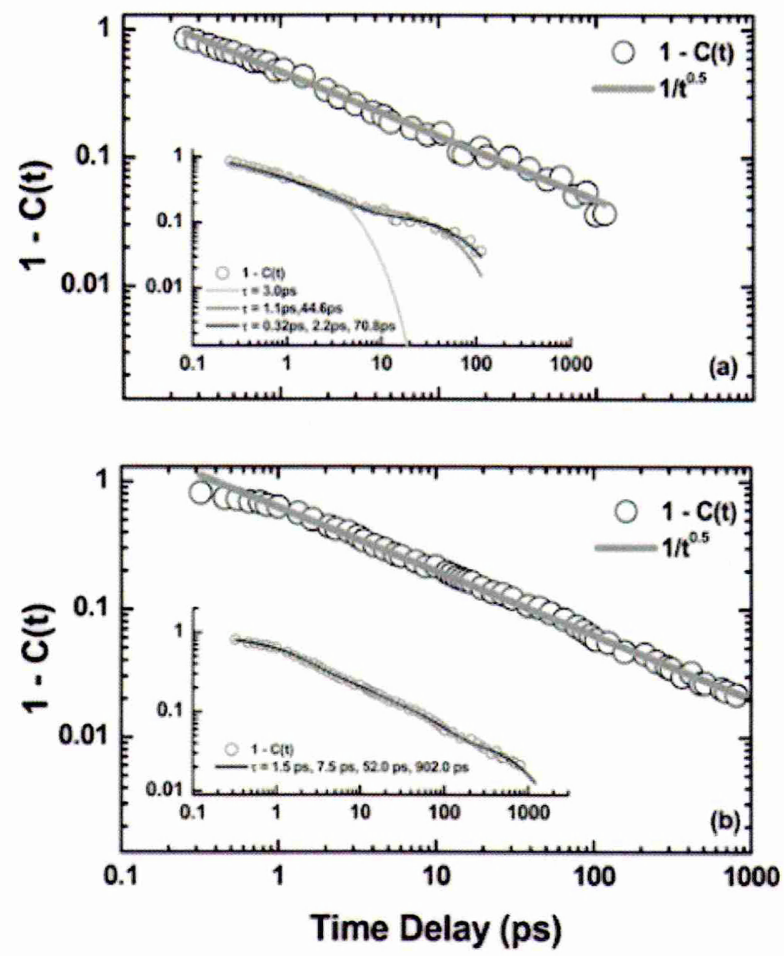

Figure 2-16: Pump probe data taken from Russo [71] on double logarithmic scale. (a) Mono, bi and tri exponential and power law $\left(t^{-1 / 2}\right)$ functions were used for the fitting and claimed that power law fits very well as compared to exponential functions for $(8,6)$. (b) Similar behaviour was observed for $(7,6)$ nanotubes and inset shows that good fit in this case requires four exponential functions.

In Fig. 2-16, the region of $t^{-1 / 2}$ behaviour extended from $<1$ ps to $\sim 1000$ ps, and there is no indication of the $t^{-1}$ behaviour reported by Ma et al. [67, 69]. Measurements were performed at a fluence of $1.0 \mathrm{~mJ} / \mathrm{cm}^{2}$, and the differential optical transmission (assumed proportional to the exciton density) decayed to $1 / 40$ of its initial value. This implies an initial 
population of at least 40 excitons per nanotube. From the absorption cross-section approach of Islam et al. [72], a maximum exciton density of $1 /(3.7 \mathrm{~nm})$ was estimated. Note that Huang et al. [70] reported measurements at similar fluences (up to $0.8 \mathrm{~mJ} / \mathrm{cm}^{2}$ ) but find a dramatically different maximum exciton density of $1 /(127 \mathrm{~nm}$ ) (3 excitons per $380 \mathrm{~nm}$ long nanotube).

Zhu et al. [73] in 2007 reported $t^{-0.45 \pm 0.03}$ power law decay in $(6,5)$ SWNTs nanotubes as shown in Fig. 2-17. They interpreted this power law decay as being due to sub-diffusive trapping of excitons in a disordered system instead of 1D diffusion limited process. We will discuss it in section 2.4.4.2.

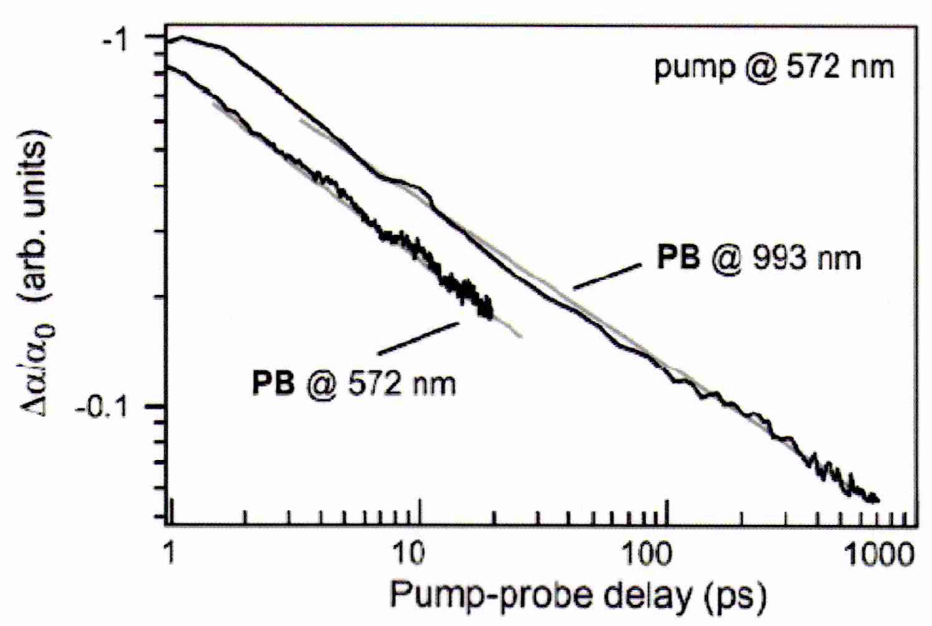

Figure 2-17: Optical transients as function of pump probe delay taken from Zhu [73]. The dynamics of photobleaching and photoinduced absorption is fitted with power law.

Recently a similar $t^{-1 / 2}$ power law decay was reported by Lüer et al. [13] for 1D diffusion of excitons in CoMoCat $(6,5)$ nanotubes. The decay was measured over a time range of a few picoseconds only, and it was assumed in the analysis that excitons annihilate immediately on contact. The main finding of this work was an exciton size of $(2.0 \pm 0.7) \mathrm{nm}$ and an exciton diffusion coefficient of $0.1 \mathrm{~cm}^{2} \mathrm{~s}^{-1}$. These are obtained from the magnitude of the intensity-dependent absorption bleaching. This work is the closest previous report to the results presented in chapter 5 of this thesis, and our measured values for these parameters are close to the results of Lüer et al. [13].

In anticipation of the discussion of our results in chapter 5, the fluences and decay behaviours reported in the above works are compared: (i) Ma et al. [67] used a fluence up to 
$\sim 4 \mathrm{~mJ} / \mathrm{cm}^{2}$ and reported a $t^{-1}$ decay at early times for HiPco SWNTs in surfactant-water system( SDS in $\mathrm{D}_{2} \mathrm{O}$ ); (ii) Russo et al. [71] used a maximum fluence of $\sim 1 \mathrm{~mJ} / \mathrm{cm}^{2}$ and observed $t^{-1 / 2}$ decay at times from $<1$ ps to $\sim 1000$ ps for SDBS-HiPco SWNTs suspension in $\mathrm{D}_{2} \mathrm{O}$; (iii) Zhu et al. [73] used a significantly smaller fluence up to $52 \mu \mathrm{J} / \mathrm{cm}^{2}$ and observed $t^{-0.45 \pm 0.03}$ decay from $<10$ ps to $\sim 800$ ps for DNA suspended CoMoCat nanotubes , and (iii) Lüer et al. [13] used a fluence of $50 \mu \mathrm{J} / \mathrm{cm}^{2}$ similar to Zhu et al. [73] and observed a $t^{-1 / 2}$ decay at early times for $(6,5)$ nanotubes in a xerogel (film).

\subsubsection{Reaction-limited and Diffusion-limited Annihilation in SWNTs}

Sutton [74] was first who reported that SWNTs act as an archetypal 1D reaction diffusion system with distinct reaction-limited and diffusion-limited regimes emerging from the same fundamental exciton-exciton annihilation process. The exciton decay shown in Fig. 2-18 clearly follows $t^{-1 / 2}$ power law decay (red line in the figure) at long times, but there is a faster decay evident at early times $<10$ ps.

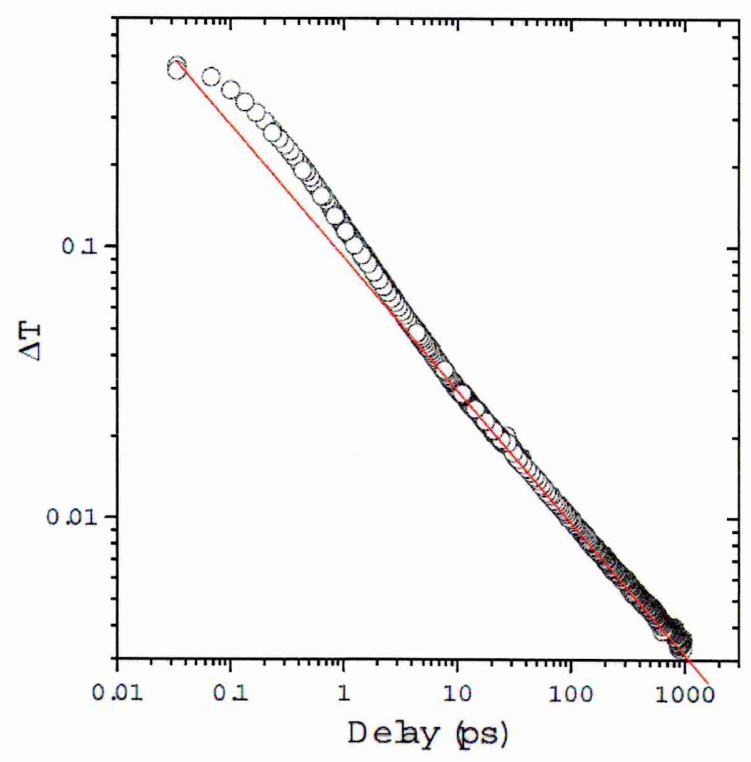

Figure 2-18: Pump probe data up to 1 ns taken from Sutton [74]. The straight red line is $t^{-1 / 2}$ power law decay.

This result is the starting point for the work described in chapter 5 of this thesis. The following aspects were unresolved in the prior work: 
1. Saturation of exciton density at high excitation intensity was observed but the origin was not understood.

2. The decay rate was faster than $t^{-1 / 2}$ at early times and high excitation densities, but never reached the $t^{-1}$ power law expected for the reaction-limited regime. There was a sign of deviation from the $t^{-1 / 2}$ power law decay around 1 ns but this was not reliably reproducible. Decay curves for different excitation densities in the long time limit were vertically displaced, in contradiction to the expectation for "universal" independence on the initial exciton population. The origin of this behaviour was not understood.

3. Parameters such as exciton density, reaction time, and diffusion coefficient were extracted on the basis of many assumptions.

4. In common with all the pre-existing work, interactions between excitons were assumed to be short-range.

\subsubsection{Effect of Trapping on Diffusive Transport in One Dimensional System including Carbon Nanotubes}

The presence of defects in one dimensional system and especially in carbon nanotubes plays important role for diffusion-limited reaction by either removing the particle from the system ('diffusive trapping') or slowing down the diffusion ('sub-diffusive trapping').

\subsubsection{Diffusive Trapping}

Diffusive trapping has been extensively studied in a medium of randomly distributed static traps. In 1D, it is one of the simplest problems which can be solved exactly. The effect of trapping on diffusive motion of a single particle was first addressed by Grassberger et al. [75] in 1982 in which they calculated the survival probabilities of a particle in a 1D system containing a random distribution of static traps. A random walker performs regular diffusion on trap-free sites and gets removed from the system upon hitting a trap i.e.

$$
A+T \rightarrow T^{*}
$$

They reported that survival probabilities of the particle at long time follows a stretched exponential of the form

$$
P(t) \propto \exp \left(-c t^{1 / 3}\right)
$$


This slow decay is explained by the presence of very large regions without any trap in which the particle can diffuse for very long times before they being trapped. A similar stretched exponential with exponent $t^{-1 / 3}$ at long time was also reported for diffusion-limited coalescence in a system consisting of segments of finite length [76]. Ma et al. [77] employed a stretched exponential similar to equation 2.21 to fit the temperature-dependent decay of exciton in carbon nanotubes, although without giving a physical justification.

Diffusive trapping with stretched exponential behaviour was also observed in polymers (plydiacetylene 4BCMU and polythiophene) by Hess et al. [78] and attributed to exciton diffusion towards recombination centres which consists of 3D distortion (defects) in the conjugated chain.

\subsubsection{Sub-diffusive Trapping (Exciton-exciton Annihilation in the Presence of Traps)}

Sub-diffusive trapping is different from diffusive trapping: instead of removing particle from the system; it slows down diffusive transport of particles and prevents the particle from interacting with each other. It has great impact on the reaction diffusion system.

The diffusive motion of particles becomes slow in the presence of traps (defects) in disordered one-dimensional system as discussed in section 2.2. In the case of strong disorder, the system may be best described by a fractal geometry (segmented regions in the case of a 1D nanotube [76]), whereas for weak disorder, the random walkers undergo sub-diffusive transport and react upon contact.

Yuste et al. [33, 35-37] invoked the mechanism of sub-diffusive trapping of particles in a disordered one dimensional system in which they studied the effect of anomalous diffusion on the kinetics of one-species coagulation and annihilation in 1D system and reported that in the long-time limit, population decays as

$$
n(t)=\frac{1}{\sqrt{2 k_{\gamma}} \Gamma\left(1-\frac{r}{2}\right)} t^{-\gamma / 2} .
$$

When $\gamma=1$, this equation reduces to normal diffusion equation for $1 \mathrm{D}$ system i.e. $n(t) \sim t^{-1 / 2}$ and for $0<\gamma<1$, the decay of reactive particles is slower than normal diffusion.

Zhu et al. [73] interpreted their observed $t^{-0.45 \pm 0.03}$ power law decay in $(6,5)$ carbon nanotubes (e.g. see Fig. 2-17) as subdiffusion-limited exciton annihilation based on the 
statement: "Survival probabilities for sub-diffusive trapping $A+\operatorname{Tr} \rightarrow \operatorname{Tr}$ "in disordered 1D systems on the other hand scale with $t^{-\gamma}$ with $0<\gamma<1$, where $t^{-1}$ would be observed for Fickian diffusion." This is erroneous (Fickian diffusion limits the reaction rate to $t^{-1 / 2}$ not $t^{-1}$ ), and their result is instead very similar to Russo et al. [71], showing normal diffusion and anomalous reaction rates (or at most very weak subdiffusion since $\gamma$ is slightly below $0.5)$.

\subsection{Saturation of Optical Response in SWNTs}

There are two different saturation effects reported for saturation of the optical response in SWNTs i.e. saturation of photoluminescence (PL) and saturation of the absorption.

\subsubsection{Saturation of Photoluminescence}

The saturation of PL can be explained by exciton-exciton annihilation which is an efficient process for removing the excitons non-radiatively and occurs on a shorter timescale than radiative recombination. The PL intensity is connected to non-radiative decay rate via

$$
I \propto \frac{k_{r a d}}{k_{r a d}+k_{n r}}
$$

where $k_{\text {rad }}$ is radiative decay rate and $k_{n r}$ is non-radiative decay rate.

Murakami et al. [50] in 2009 presented the nonlinear photoluminescence excitation of SWNTs and reported the saturation of PL at high pump fluences independent of excitation wavelength and nanotube chirality. This saturation is explained by diffusion-limited excitonexciton annihilation in SWNTs.

The saturation of PL intensity has also been observed $[39,51]$ in air suspended SWNTs where the emission saturates even for as few as 2-6 excitons per nanotube. The estimated exciton densities for highly saturated regimes are much lower than the expected Mott density.

\subsubsection{Saturation of Exciton Population}

Excitons are photogenerated by spin-1 photons and exhibit bosonic characteristics at low densities. However the exciton is best described as a composite fermion (details are described 
in ref [79]) where the constituent electrons and holes are weakly coupled by the Coulomb interaction rather than through quantum-mechanically entanglement. As a result, the maximum density is limited by the Pauli Exclusion principle which stated that the transitions into occupied final states are forbidden. Since this maximum density is approximately equal to the inverse of the exciton size, hence it is orders of magnitude higher that the density where PL saturation occurs, which is approximately equal to the inverse nanotube length.

This saturation effect in SWNTs was first invoked in 2003 by Arnold et al.[80] in order to explain the sublinear behaviour of the photobleaching signal at high pump intensities. This saturation was attributed to state filling effect. Recently the saturation of initial population of excitons at a concentration of $\sim 1 /(2 \mathrm{~nm})$ in CoMoCat $(6,5)$ nanotubes was reported by Lüer $e t$ al. [13] and attributed to Pauli repulsion of the excitons.

\subsection{Plasmonic Contribution to Optical Response in Carbon Nanotubes}

Without specific effort to select tubes of a specific diameter or chirality, solutions of nanotubes typically contain a range of sizes and species, and hence the absorption spectrum consists of numerous peaks. These are typically superimposed on a broad background which has been attributed to the tail of a $\pi$-electron surface plasmon which in graphene is resonant at $\sim 5 \mathrm{eV}[81,82]$ The origin of this plasmonic background has been alternatively attributed to a residual graphitic background $\left(\mathrm{C}_{60}\right.$, or amorphous carbon) or may be due to metallic nanotubes [83].

Lauret et al. [84] have probed the optical response between excitonic features in a solid sample of SWNTs on glass substrate and observed photoinduced absorption with an approximately exponential decay ( 1 ps decay time) which they associated with excitation and decay of the plasmon, shown in Fig. 2-19 (a).

However, recent progress has been made towards the purification and isolation of SWNTs using polymers e.g. PFO [85-88] which show strong chirality and diameter selectivity. The resultant SWNT-polymer mixture contains only a few nanotube species and the linear optical absorption shows almost complete removal of the background as shown in Fig. 2-19 (b). This may be due to the removal of graphitic impurities or metallic nanotubes. In either case it indicates that the 'plasmon' background is not an intrinsic feature of semiconducting nanotubes. 

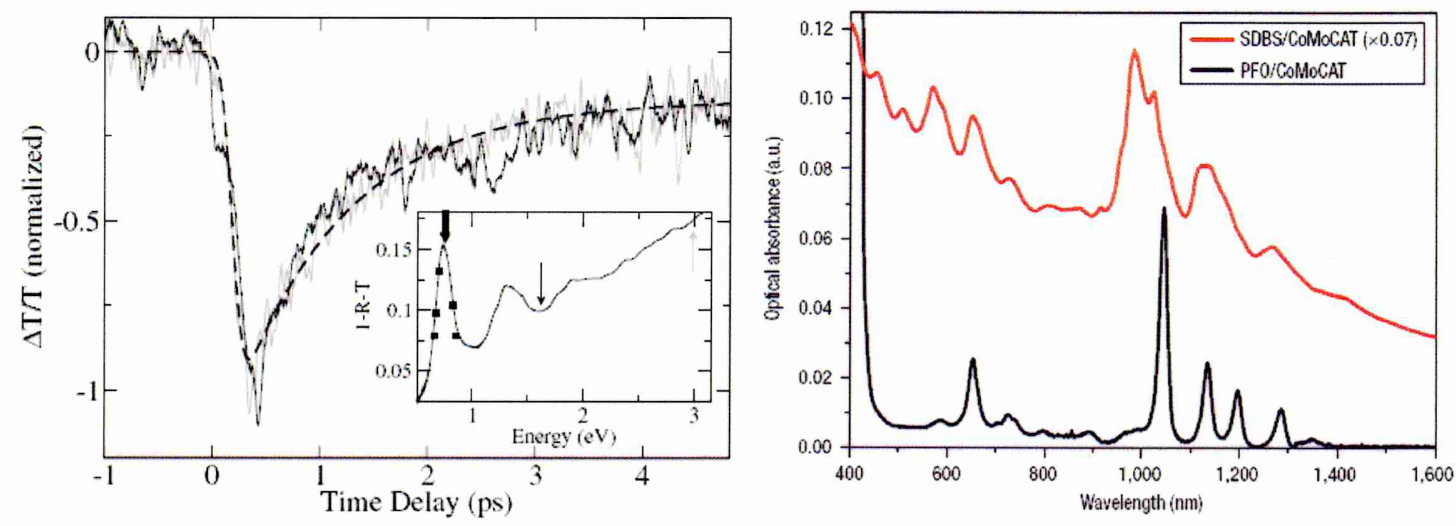

Figure 2-19:- (a) The non resonant pump probe measurement of SWNT taken from Lauret [84] is showing monoexponential decay of 1 ps. (b) optical absorption spectra of CoMoCat SWNTs obtained through different separation method. This picture is adapted from [88]. 


\section{CHAPTER 3}

\section{EXPERIMENTAL TECHNIQUES}

In this chapter, we describe the main tools and techniques which were used in our experiments. Firstly, a brief overview of the ultrafast laser system used to measure the optical properties of SWNTs will be given followed by a general description of the pump probe technique which is the main technique used in obtaining the results presented in this thesis. Finally, we will finish the chapter with a brief overview of spectrally resolved pump probe measurements and the generation of white light (broadband) probe pulses.

\subsection{The Amplified Titanium-doped Sapphire Ultrafast Laser System}

The availability of tuneable high intensity laser pulses with duration of significantly less than a picosecond allows one to create and study short lived transient states in materials. Titanium-doped sapphire (Ti:sapphire) is the most common laser material used for this purpose because of its broad gain bandwidth from 700 to $1100 \mathrm{~nm}$ peaking around $800 \mathrm{~nm}$, extremely good thermal conductivity, high gain cross section, and high Kerr coefficient [89].

In order to study the nonlinear optical properties of SWNTs which have electronic transitions in the infrared at wavelengths beyond those emitted by Ti:sapphire, we use a nonlinear wavelength converter: an optical parametric amplifier (OPA) driven by an amplified Ti:sapphire laser system. The Coherent ${ }^{\left({ }^{\mathrm{T}}\right)}$ RegA laser system [90] provides sufficient peak intensity to drive the OPA at repetition rate up to $250 \mathrm{kHz}$. For our experiments, this proves a suitable intermediate solution between unamplified $\sim 100 \mathrm{MHz}$ systems, which require tight focussing of the light spot to achieve the required optical intensity to observe exciton saturation and which cause substantial optical heating of the sample, and low repetition rate $1 \mathrm{kHz}$ systems where optical damage to the sample can be hard to avoid.

The schematic of the ultrafast laser system is shown in Fig. 3-1. This ultrafast laser system consists of several units; a brief description of each unit is given below. 


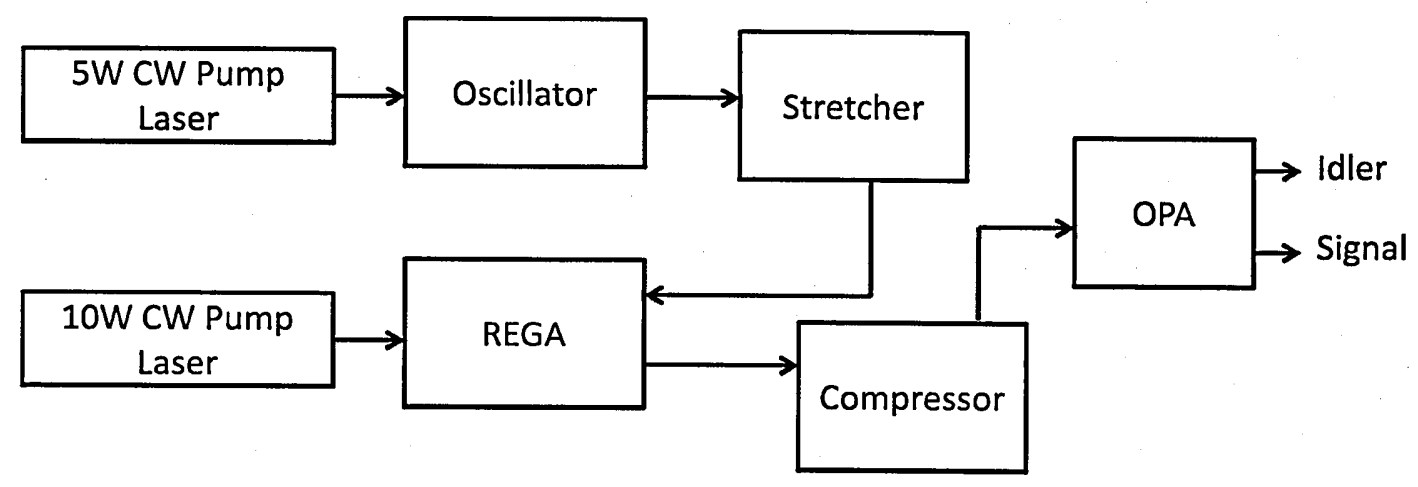

Figure 3-1: Schematic of ultrafast laser system. The brief description of each unit is given in the text. $R E G A=$ Regenerative amplifier, $O P A=$ Optical parametric amplifier.

\section{Laser Oscillator}

The laser pulses are generated in a Coherent 'Mira' mode-locked Ti:sapphire laser pumped by a $5 \mathrm{~W}$ solid-state diode laser. This is configure as a seed oscillator emitting at a fixed wavelength of $800 \mathrm{~nm}$ for subsequent amplification. A passive mode-locking technique (Kerr lens mode-locking) [90] and compensation of group velocity dispersion using a prism sequence are used to generate pulses of duration (full-width at half maximum, FWHM) $\sim 60$ fs.

\section{Chirped Pulse Amplification}

The further amplification of optical pulses with short duration and high peak powers is limited by the damage threshold of most optical materials. This problem is overcome in chirped pulse amplification (CPA) scheme by stretching the pulses in time using group velocity dispersion in a pair of optical gratings. The stretched pulse is then amplified, before being recompressed to its original pulse duration.

Pulses with 100 ps duration are produced in the stretcher and these pulses are sent to a Coherent 9050 REGA (regenerative amplifier) for the amplification. The $6 \mathrm{~nJ}$ stretched seed pulse from the $80 \mathrm{MHz}$ pulse train is amplified through CW-pumped Titanium doped sapphire crystal via 26 multipasses. Ti-sapphire is an ideal amplifier gain material because of its long storage time ( $3 \mu \mathrm{s})$ and large saturation fluence $\left(1 \mathrm{Jcm}^{-2}\right)$. In REGA, the energy needed for this regenerative amplification process is taken from a $10 \mathrm{~W} \mathrm{CW}$ pump beam 
(Coherent Verdi 10). Pulses with energy approximately $6 \mu \mathrm{J}$ at $800 \mathrm{~nm}$ are generated and the repetition rate of the pulses can be changed between $10 \mathrm{kHz}$ and $300 \mathrm{kHz}$

The pulses in the compressor are then recompressed to $\sim 60 \mathrm{fs}$ ultrashort pulses. The design of the compressor is a major consideration in a femtosecond laser system because the stretcher-compressor is most dispersive part of the system. Any mismatch between stretcher and compressor causes third and fourth order contributions to the phase expansion. However, the compressor is separately adjusted to correct the dispersion introduced by the amplification process in the REGA.

\section{OPA (Optical Parametric Amplifier)}

The ultrashort pulses from compressor are sent to an optical parametric amplifier for wavelength conversion. Inside the OPA, white light continuum pulses are generated via self phase modulation by focussing the $\sim 60 \mathrm{fs}$ pulses onto a sapphire crystal. The remnant $800 \mathrm{~nm}$ beam and this white light are then focussed onto BBO ( $\beta$-Barium borate) which has a tuning range from 1.1-2.8 $\mu \mathrm{m}$ and a high damage threshold. The signal pulses (1.1-1.6 $\mu \mathrm{m})$ and perpendicular polarised idler pulses (1.6-2.4 $\mu \mathrm{m})$ are generated via parametric amplification [89]. The wavelength of the signal and idler pulses can be changed by changing the angle of BBO crystal [89], which alters the phase-matching condition for the optical parametric amplification process. Pulses with $150-200 \mathrm{~mW}$ power and approximately $60 \mathrm{fs}$ (FWHM) pulse duration are generated and these pulses are then sent to the experiment.

\subsubsection{Definition of Beam and Pulse Parameters}

Here we discuss some important beam parameters and quantities derived from these parameters which define the spatial and temporal variation of ultrashort pulses. These parameters and quantities will be used later on in this thesis. The pulses produced by Coherent ${ }^{(\mathrm{TM})}$ laser system have approximate Gaussian profile and the radial profile of pulses is best described by the fundamental Gaussian $\mathrm{TEM}_{00}$ mode. The intensity distribution is described by

$$
I(r, z)=I_{0}\left(\frac{w_{0}}{w(z)}\right)^{2} e^{\frac{-2 r^{2}}{w(z)^{2}}}
$$

where $I_{0}=I(0,0)$ is intensity at centre of beam, $w_{0}$ is radius of the beam at focal point 
and is also called spot size, $r$ is radial coordinate and $w(z)$ is beam radius which varies along beam direction and is define as point at which intensity falls to $1 / e^{2}$ of its maximum value i.e.

$$
w(z)=w_{0} \sqrt{1+\left(\frac{\lambda z}{\pi w_{0}^{2}}\right)^{2}}
$$

The confocal length " $z_{0}$ " is the distance over which beam expands by a factor of $\sqrt{2}$ and is given by

$$
z_{0}=\frac{\pi w_{0}^{2}}{\lambda}
$$

The spot size at sample was measured by translating Razor blade and whilst the transmission of power was measured as function of position. It has been reported [91] that the beam radius can be determined by

$$
w_{0}=0.552 \sqrt{2}\left|x_{10}-x_{90}\right| .
$$

where $x_{10}$ and $x_{90}$ are positions of Razor blade corresponding to $10 \%$ and $90 \%$ transmission of the beam respectively.

In order to measure the energy density, we need to measure the pulse energy. The pulse energy at the sample is given by

$$
\text { Pulse energy }(J)=\frac{\text { pump power }(J / s)}{\text { repetition rate }(1 / \mathrm{s})} \text {. }
$$

Then energy density at place of the sample is determined from pulse energy as

$$
\text { Energy density }\left(J / \mathrm{m}^{2}\right)=\frac{\text { pulse energy }(J)}{\text { Area of } \operatorname{spot}\left(\mathrm{m}^{2}\right)}
$$

In ideal case, illuminated area of the sample is $\pi w_{0}^{2}$ (for circular beam) and above equation 3.6 becomes

$$
\text { Energy density }\left(J / \mathrm{m}^{2}\right)=\frac{\text { pulse energy }(J)}{\pi w_{0}^{2}\left(m^{2}\right)}
$$

We calculated the pump fluence from energy density as

$$
\text { Pump fluence }\left(\frac{\text { photon }}{\mathrm{m}^{2}}\right)=\frac{\text { energy density }\left(\frac{\mathrm{J}}{\mathrm{m}^{2}}\right)}{\hbar\left(\mathrm{J} \cdot \mathrm{s}^{-1}\right) * \omega\left(\mathrm{s}^{-1}\right)} \text {. }
$$




\subsection{Pump Probe Technique}

The pump probe technique is a common technique used in ultrafast spectroscopy. We used the standard degenerate pump probe technique to obtain the information about the decay of excitons in SWNTs. Generally, the relatively strong pump beam excites the sample and initiates some processes of interest for example in our case, the creation of non-equilibrium carrier distribution in the SWNT samples. The probe beam which enters the sample after some adjustable time delay (controlled by optical delay line), its transmission (reflection) will be attenuated or amplified because of changes induced by pump pulse.

The information about the process initiated by the pump beam can be obtained by monitoring the probe signal as function of time delay $(\tau)$. A fast photodetector is not required as the probe signal is averaged over many pulses. Therefore, the temporal resolution of our experiment is fundamentally limited only by pulse duration. A pump probe signal measured in this work is shown in Fig. 3-2 which shows the pump induced change in probe transmission $(\Delta \mathrm{T})$ as function of time delay $(\tau)$.

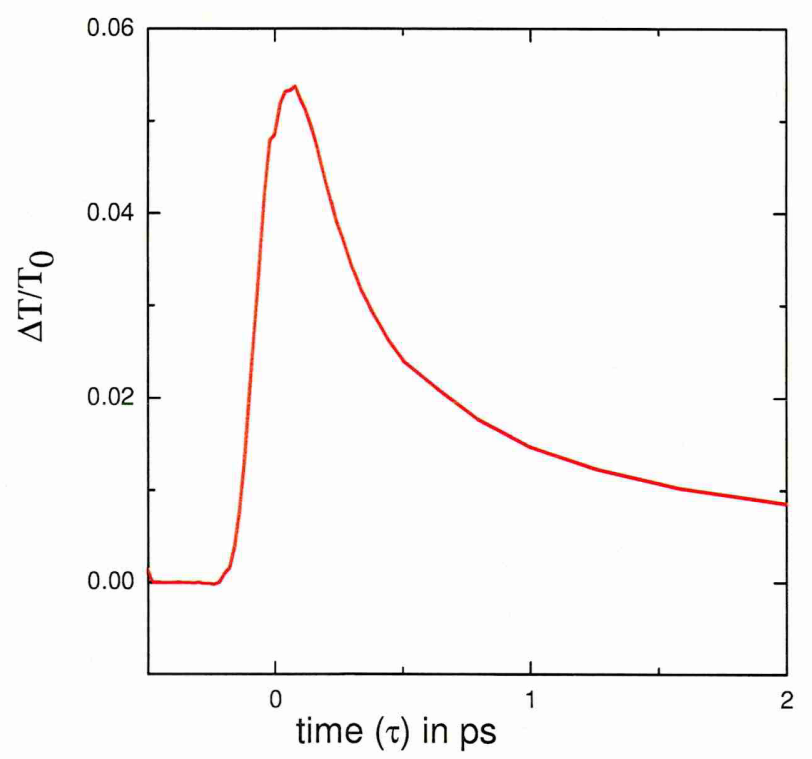

Figure 3-2: Pump probe trace as function of pump probe delay. The detail about the trace is given in text.

For negative time delay $(\tau<0), \Delta \mathrm{T}=0$ which indicates that the pump beam has not induced any change in the SWNT sample yet. Around $\tau=0, \Delta \mathrm{T}$ increases sharply as the 
pump pulse arrives which indicates that the pump pulses have created a non-equilibrium excited carrier distribution in the sample. The magnitude of $\Delta T(0)$ increases with increasing intensity of the pump pulse (see chapter 5 or 6 ). For a positive time delay $(\tau>0)$, pump induced signal $(\Delta \mathrm{T})$ decays with time delay which means that pump induced excited carrier population are decaying back to the equilibrium state through various mechanisms which can be radiative or non-radiative or both depending on nature of material being investigated. According to the work reported in the literature (discussed in chapter 2), the exciton dominates the processes that govern the return to equilibrium of optically excited SWNTs.

\subsubsection{Experimental Setup}

The setup of our degenerate pump probe experiment is shown in Fig. 3-3. The pump pulses are sent through a computer controlled variable delay line consisting of Gold-plated retroreflector mounted on linear delay stage (Melles Griot 17 NST-Motorized long-travel linear stage) which has $150 \mathrm{~mm}$ scan range, maximum speed of $6 \mathrm{~mm} / \mathrm{sec}$, and $50 \mathrm{~nm}$ resolution. This delay in the path of pump beam is matched by introducing an additional path length in probe beam so that both beams can reach simultaneously onto the sample within the scan range of the delay stage.

The change in delay $\Delta \tau$ due to translation stage $\Delta x$ is approximately given by

$$
\Delta \tau=2 \times \frac{\Delta x}{c}
$$

where $c=3 \times 10^{8} \mathrm{~m} / \mathrm{sec}$ is speed of light.

The delay range which can be achieved in our experiment with single pass of delay line is 1000 ps with a maximum time resolution of 0.3 fs. This resolution is far in excess that is required for our experiment.

The data for the CoMoCat sample was taken by focussing the pump beam onto the sample using a $200 \mathrm{~mm}$ focal length lens and the probe beam using a $125 \mathrm{~mm}$ lens. In the case of HiPco sample, the pump and probe beams were focussed using $400 \mathrm{~mm}$ and $300 \mathrm{~mm}$ lenses respectively. The different focal length lenses were used to change the excitation intensity. The larger focal length of the pump beam results in a larger spot size which excites a large area of the sample. The probe beam was focussed at the centre of the pump spot so that it probes an approximately uniformly-excited area of the sample. The change in transmission 
$(\Delta \mathrm{T})$ of the probe beam was measured by focussing the probe beam onto a balanced photodiode detector which was connected to a lock-in-amplifier as shown in Fig. 3-3.

A non-collinear pump probe technique was used in which the pump and probe beams were focussed onto the sample at an angle of approximately $17^{\circ}$ to each other. This angle was chosen to minimise the contribution of pump scattering to the measured signal and will be discussed in detail in section 3.2.1.2. The temporal resolution in our experiment is limited by duration of the pulse as the resolution achieved with the variable delay line is 0.3 fs. Some further changes have been made in the previous setup (described in [74]) to improve the signal to noise ratio. These improvements are described below.

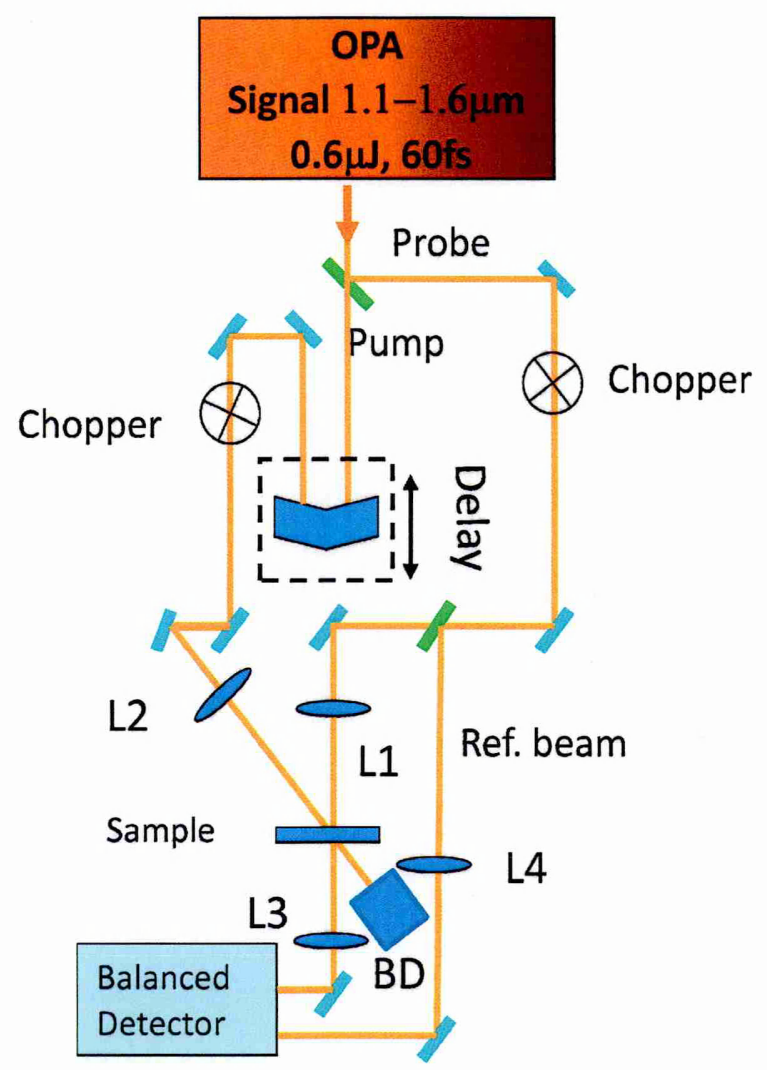

Figure 3-3: Experimental setup used to obtain the pump probe results presented in this thesis. BD is beam dump and L1, L2, L3, L4 are lens 1, lens2, lens3, lens 4 respectively.

\subsubsection{Improvement of SNR}

It is important to improve the signal to noise ratio especially in the case of low excitation and at longer times. This signal to noise ratio was improved by (1) minimising the amount of 
noise amplified by the lock-in-amplifier. The amount of noise amplified by the lock-inamplifier depends on detection bandwidth which is set by the equivalent noise bandwidth (ENBW) of the low pass filter. In our experiments, the slope of $18 \mathrm{~dB} /$ oct and time constant of $T=300 \mathrm{~ms}$ were selected which correspond to

$$
\mathrm{ENBW}=\frac{3}{32 \mathrm{~T}}=0.31 \mathrm{~Hz}
$$

This is the bandwidth over which the broadband noise is amplified in our experiments [92].

(2) Taking multiple scans ( $\sim 50$ scans) for each measurement. This is needed for accurate measurement of the background signal as well as for the determination of standard deviation and standard error in the mean.

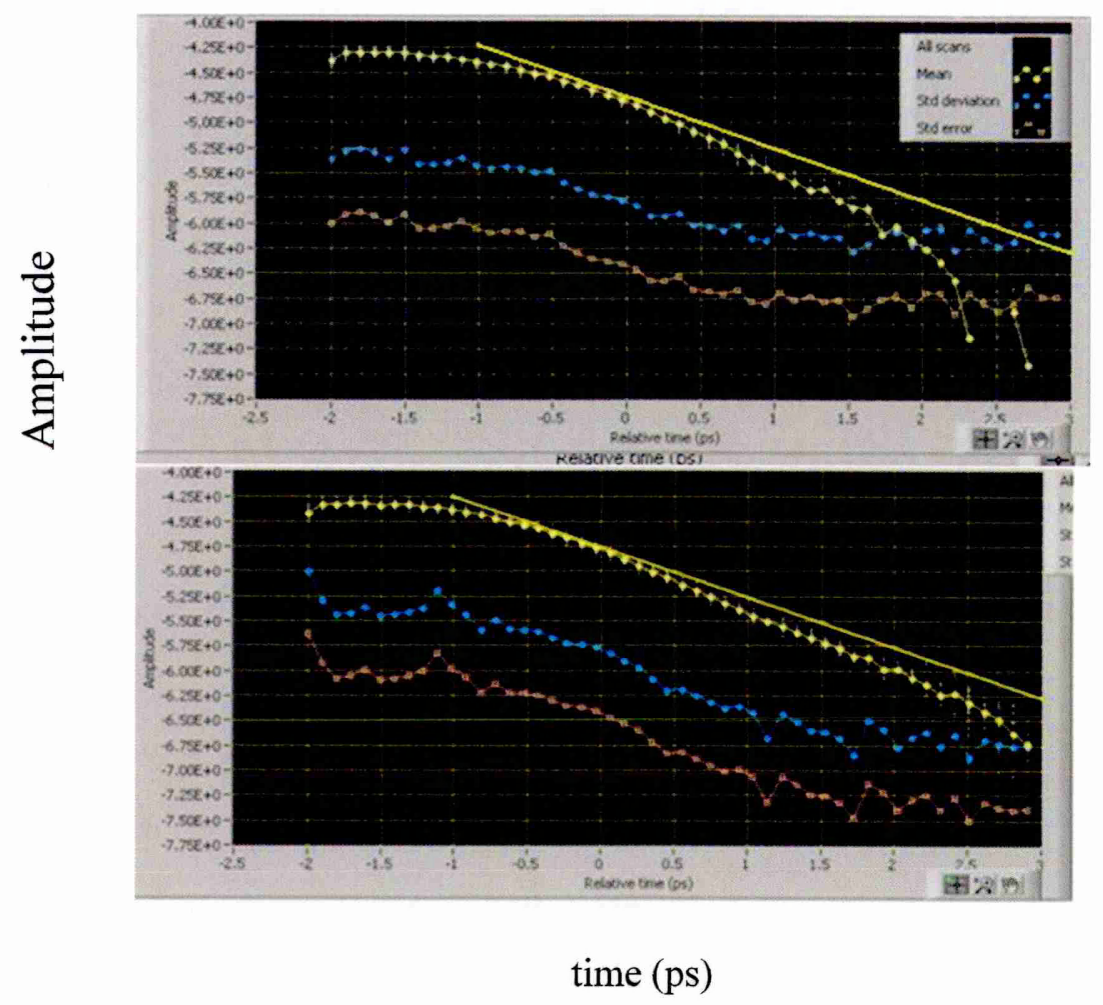

Figure 3-4: Comparison of SNR for the case of unbalanced and balanced detector. Top graph was taken with unbalanced detector and bottom one with balanced detector.

(3) Further improvements were made by rejecting the common mode noise of the laser. This was done by sending the reference probe beam and the probe beam passing through the sample onto a balanced photodiode detector (differential detector) which subtracts the reference beam from the sample probe beam and sends the difference output to the lock-in 
amplifier. The comparison of SNR for the balanced and unbalanced case is shown in Fig. 3-4.

\subsubsection{Rejection of Scattered Signal}

After removing the common mode noise of the laser, the main contribution of noise in measured signal comes from scattering of the pump beam. The scattering of pump light by the aqueous suspension is inevitable. It is minimised if the presence of precipitates is minimised. Scattering occurs into whole solid angle, so minimising the solid angle 'seen' by the detector can help in minimising the scattered signal. In Fig. 3-5, comparison of two different values of angles between pump and probe beam is shown.
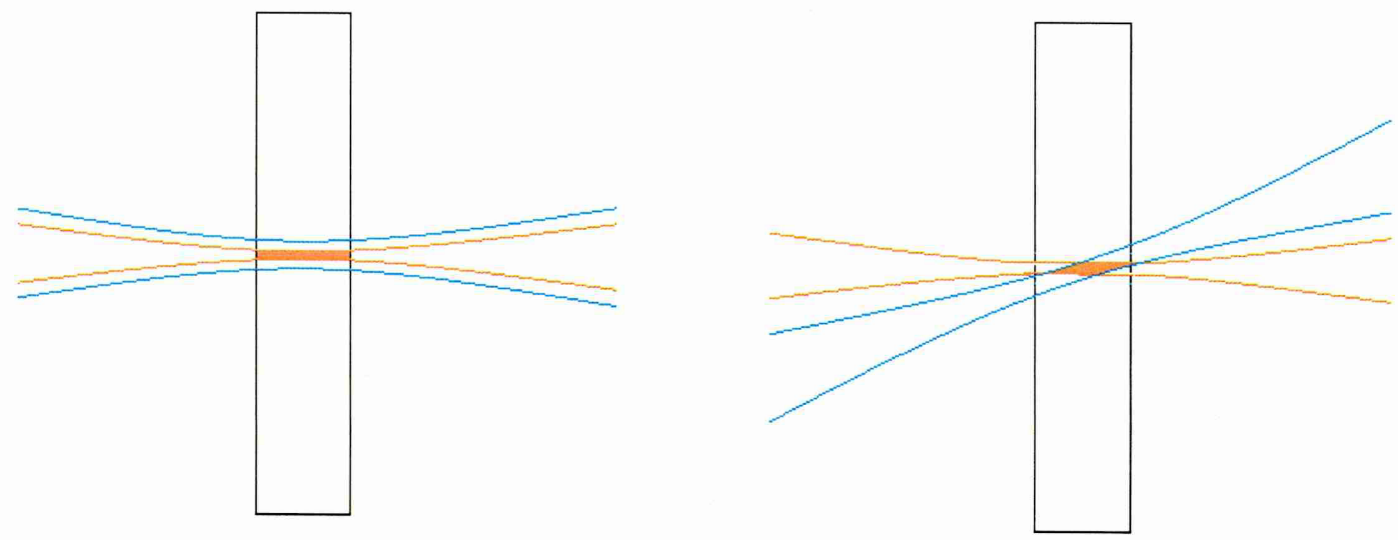

Figure 3-5: Schematic of pump probe overlap (a) when the angle between pump and probe is $0^{\circ}(b)$ when the angle is $\theta \sim 20^{\circ}$. The detail is given in text.

The Fig. 3-5 shows that when the angle is small i.e. $\theta=0$, SNR will be low because of large scattering of pump beam and when the angle is large i.e. $30^{\circ}<\theta<90^{\circ}$, it is hard to find the signal because of small overlapping area. Another disadvantage of large angle is that it causes the degradation of times resolution. As a result, an angle of $\theta=17^{\circ}$ between pump and probe was selected, which is large enough to separate the beams given their divergence. The transversal time (propagation time of pulse) of $120 \mathrm{fs}$ was calculated using measured beams waist and $\theta=17^{0}$ via

$$
t=\frac{s}{c} \times \varepsilon_{w} \times \sin \theta
$$

where $\varepsilon_{w}$ is refractive index of water, $s$ is correlation of pump and probe waist, and $c$ is 
speed of light.

Further reduction was made by placing an iris after the sample which allows probe pulses to pass only because of non-colinearity of the two beams. Furthermore, the pump beam was sent to a beam dump after passing through the sample in order to avoid its further contribution from reflection or scattering onto detector.

\subsubsection{Equally Spaced Data Points (Log Scan)}

Another improvement was made by choosing the electronic steps of the translation stage in such a way that equally spaced data points can be collected. The equally spaced data points are needed as the data follows a power law (a power law on log-log scale is linear which gives equal importance to data at early time as well as at long time). Also least square fitting can be applied to data which is evenly spaced on logarithmic axes. Prior to this, data was collected on linear axes which collect more points at early times and fewer points at long times which affects the analysis.

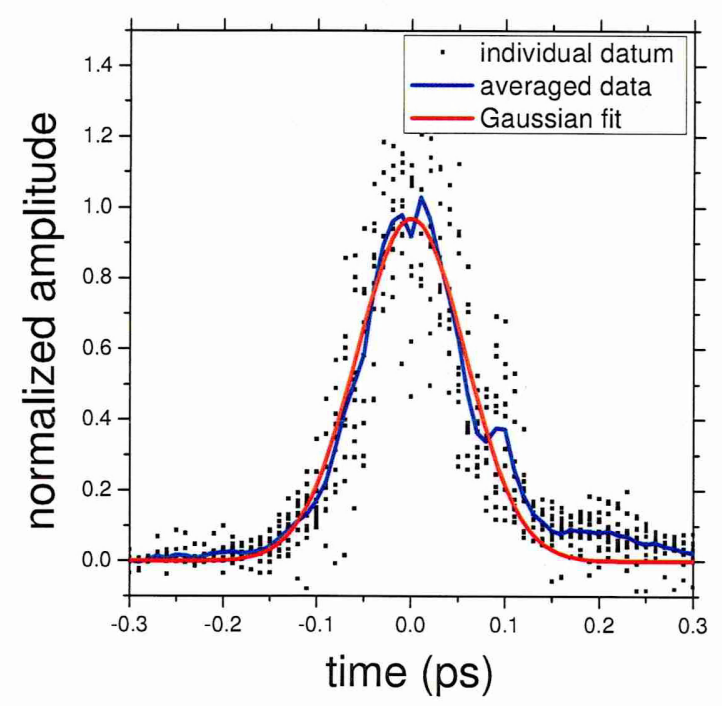

Figure 3-6: Standard error in mean, appeared due to interference fringes of beams, was used to measure position of time zero and pulse width. Black dots are standard error in mean of individual pump intensities used, blue line is mean value of all standard error in mean, and red line is the fit using a Gaussian function. 


\subsubsection{Correct Measurement of Time Zero Position}

The accurate measurement of the time zero is a key to extract the correct information about early dynamics in our measurement. The position of the time zero was measured from peak which appeared in standard error of the mean due to interference fringes of the beams as shown in Fig. 3-6. This correct measurement of time zero not only improves our understanding of dynamical processes but also shows that exact measurement of the time zero is very important and key for these kinds of experiments as it will be discussed in chapter 5 . The pulse width was also measured from standard error of the mean by fitting it with a Gaussian function as shown in Fig. 3-6. A pulse width of $\sim 135 \mathrm{fs}$ was measured which is slightly higher than the value of $\sim 90$ fs (when the angle is zero) calculated from correlation of pump and probe beams. This is due to the large angle between pump and probe beams which causes the degradation of times as discussed in 3.2.1.2. Thus actual temporal resolution of our experiment is limited by this pulse width ( $\sim 135 \mathrm{fs})$.

\subsubsection{Alignment of Delay Stage}

In order to obtain accurate results, the pump beam must remain focussed on same spot when the delay stage is moving i.e. the overlap of the pump and probe beams remain constant throughout the experiments. This requires that pump beam must be parallel to the axis of delay stage. This parallelism was checked by sending the pump beam after the delay stage through convex lens of $1 \mathrm{~m}$ focal length, to far field around $3 \mathrm{~m}$ away. When the beam is parallel to the travel of the stage, no translation of beam is observed because it always hits the same point on the lens for all delay stage positions and when it is not parallel, a large translation is observed.

\subsubsection{Dual Chopping Configuration}

For the pump probe measurements discussed in this thesis, a Stanford research model SR 830 DSP lock-in-amplifier was used. In order to improve SNR, we modulated both the pump and probe beams at $f_{1}(300 \mathrm{~Hz})$ and $f_{2}(500 \mathrm{~Hz})$ respectively by using a mechanical chopper. The reference input of the lock in amplifier was connected to the sum output $\left(f_{1}+f_{2}\right)$ of the chopper which detects the signal modulated at $f_{1}+f_{2}$ only and rejects all other.

For negative delay i.e. $\tau<0$, the probe beam passes through the sample and detector 
unmodulated by pump pulse, therefore it is not detected by the lock-in -amplifier. For positive delay i.e. $\tau>0$, the transmission of the probe pulse is changed due to presence of pump beam

$$
T_{p r}=T_{0}+\Delta T(\tau)
$$

where $\Delta T(\tau)$ is change in transmission caused by the pump beam. The lock in amplifier detects this nonlinear change in transmission. Furthermore, the linear transmission " $T_{0}$ " of the sample was also measured by blocking the pump beam and chopping the probe beam at $\mathrm{f}_{2}$ $(500 \mathrm{~Hz})$ with the reference input of the lock-in-amplifier connected to $f_{2}$ (reference output of the chopper). This is necessary as the absolute value of $\Delta T / T_{0}$ is plotted as a function of pump probe delay in this thesis.

\subsubsection{Quantitative Interpretation of Pump Probe Spectroscopy}

The change in transmission $\Delta T / T_{0}$ of probe beam can be found from the fractional change in absorption. For an unsaturated signal (unsaturated population), the fractional change in intensity " $\frac{d I}{I}$ " of the probe beam as it passes through the sample neglecting the reflection and scattering is given by

$$
\frac{d I}{I}(z)=\left[-\partial-\sigma N_{0} e^{-\partial z}\right] d z
$$

In equation 3.13, the first and second terms are included for linear absorption and absorption from pump-induced excited species respectively. The fractional change in transmission $\Delta T / T_{0}$ of probe beam due to the pump beam can be obtained through the integration of equation 3.13 and is given by

$$
\frac{\Delta T}{T_{0}}=\exp \left[-\frac{\sigma N_{0}}{\partial}\left(1-e^{-\partial l}\right)-\partial l\right]
$$

It is important to consider the thickness of sample. For thin sample i.e. when $\partial l \ll 1$, equation 3.14 becomes

$$
\frac{\Delta T}{T_{0}} \approx-\sigma N_{0} l
$$

For thick sample i.e. when $\partial l \gg 1$, it becomes 


$$
\frac{\Delta T}{T_{0}} \approx-\frac{\sigma N_{0}}{\partial}
$$

For saturation of population, the equation 3.13 should be modified.

\subsection{White Light Generation and Spectrally Resolved Pump Probe Measurements}

The spectrally resolved pump probe measurements on SWNTs were performed by probing multiple wavelengths simultaneously using white light continuum (broadband) probe pulses. The results of spectrally resolved measurements are given in chapter 7 .

The white light probe pulses were generated through a physical mechanism called self phase modulation [89] which occurs when intense laser beam as produced by our laser system propagates through a non-linear media. The white light probe pulses were generated by focussing regenerative amplifier (REGA) $800 \mathrm{~nm}$ pulses of $\sim 60 \mathrm{fs}$ duration onto a $3 \mathrm{~mm}$ thick sapphire crystal using a lens of $10 \mathrm{~cm}$ focal length $(650-850 \mathrm{~nm})$. A schematic of the generation of white light continuum pulses is shown in Fig. 3-7. Long pass filter was used after the sapphire crystal in order to remove the remnant $800 \mathrm{~nm}$ radiation from the white light continuum. It was found that pulse energy of $1.2 \mu \mathrm{J}$ is sufficient to generate an efficient white light continuum pulse that is spectrally broad enough to cover the $E_{11}$ transitions of nanotube species present in our sample. Furthermore, it is also in the range of previously reported values of $0.5-2 \mu \mathrm{J}$ used for generation of white light continuum in sapphire [93].

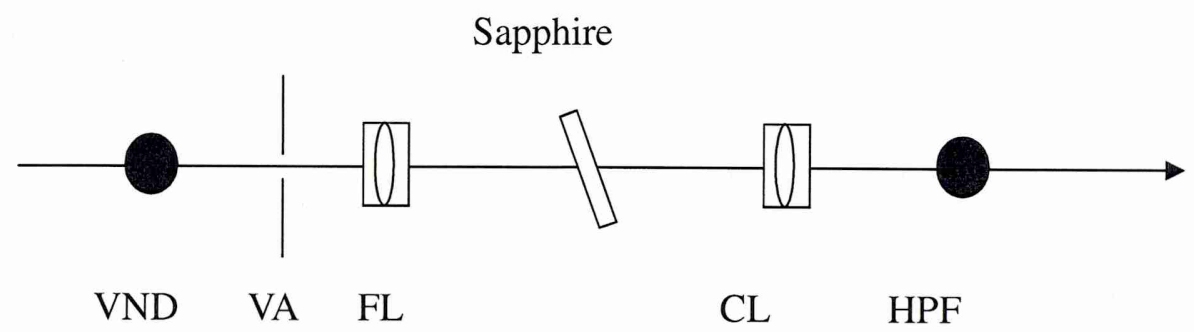

Figure 3-7: Schematic of experimental setup of generation of white light continuum probe pulses. $V N D=$ variable neutral density filter, $V A=$ variable aperture to adjust the beam profile, $F L=10 \mathrm{~cm}$ focussing lens, $C L=5 \mathrm{~cm}$ collimating lens, HPF $=$ high pass filter to remove the remnant $800 \mathrm{~nm}$ laser beam from the white light. Sapphire was mounted on translation stage with micrometer adjust in all three direction. 
Another important thing which was used to improve the efficiency of the white light continuum is an aperture in front of the sapphire crystal which controls the beam profile, whereas phase matching condition was achieved through the crystal angle. This white light was then reflected through a mirror towards a Jobin Yvon iHR550 spectrometer, where it was detected by liquid nitrogen-cooled InGaAS photodiode array and further optimisation (e.g. slit widths etc) was done using the Symphony software.

Once the white light was generated, some tests were performed to improve its efficiency using different beam splitters, focusing lenses, and repetition rates. It was found that a $10 \mathrm{~cm}$ focussing lens and $100 \mathrm{kHz}$ repetition rate can generate more efficient white light continuum pulses needed for probing the energy range of $E_{11}$ transitions of SWNTs. The spectrum of optimised white light continuum is shown in Fig. 3-8.

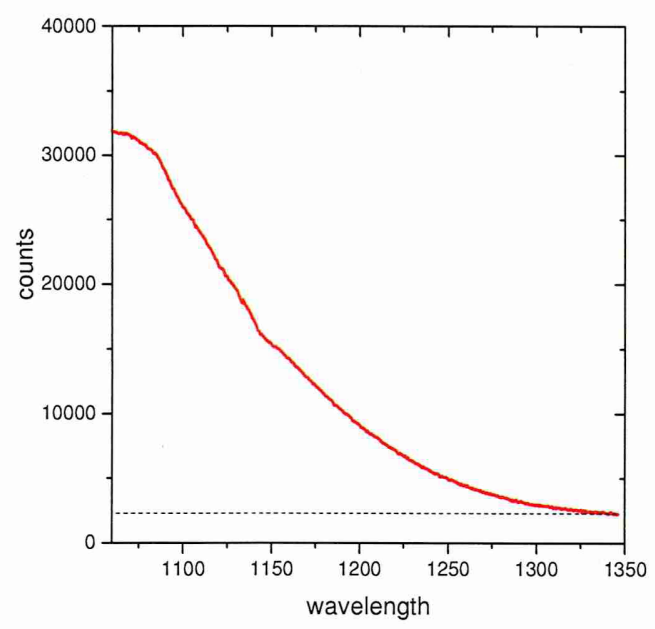

Figure 3-8: The optimised white light spectrum generated at the repetition rate of $100 \mathrm{kHz}$ using a $10 \mathrm{~cm}$ focussing lens. The spectrum is broad enough to probe the $E_{11}$ transitions of SWNTs.

This white light was used as probe pulse for the spectrally resolved pump probe experiments as shown in the Fig. 3-9. Finally a beam splitter with $60 \%$ reflection and $40 \%$ transmission was used to split the $800 \mathrm{~nm}$ regenerative amplifier (REGA) output, $60 \%$ of amplifier output was used to generate white light continuum probe beam and $40 \%$ was used to pump the optical parametric amplifier (OPA).

After the generation of white light probe pulses, time zero was found using Jobin Yvon iHR550 spectrometer as monochromator and focussing the beam onto a Thorlab InGaAs detector. The same alignment procedure was followed as for the standard pump probe 
measurements.

For spectrally resolved measurements, the $E_{11}$ of SWNTs was pumped using the output of OPA and probed with the white light continuum over the wavelengths $1050-1350 \mathrm{~nm}$ which covers almost whole range of $E_{11}$ transitions of nanotube species. Photoinduced transmission $\Delta T(\lambda, \tau)$ for different delays and different pump intensities were measured i.e.

$$
\begin{gathered}
\Delta T(\lambda, \tau)=\Delta T_{\text {Positive time }}-\Delta T_{\text {negative time }} \\
\Delta T(\lambda, \tau)=T(\lambda, \tau)-T_{0}(\lambda) .
\end{gathered}
$$

where $T(\lambda, \tau)$ is pump-induced probe transmission change detected by InGaAs array attached to spectrometer and $T_{0}(\lambda)$ is probe transmission spectrum, measured by taking the spectrum at negative time delay. The spectrally resolved measurements presented in chapter 7 were taken by subtracting the transmission spectra measured at positive time delay and negative time delay. Then these spectrally resolved data was combined to obtain the $2 \mathrm{D}$ contour plot of $\Delta \mathrm{T}(\lambda, \mathrm{t})$.

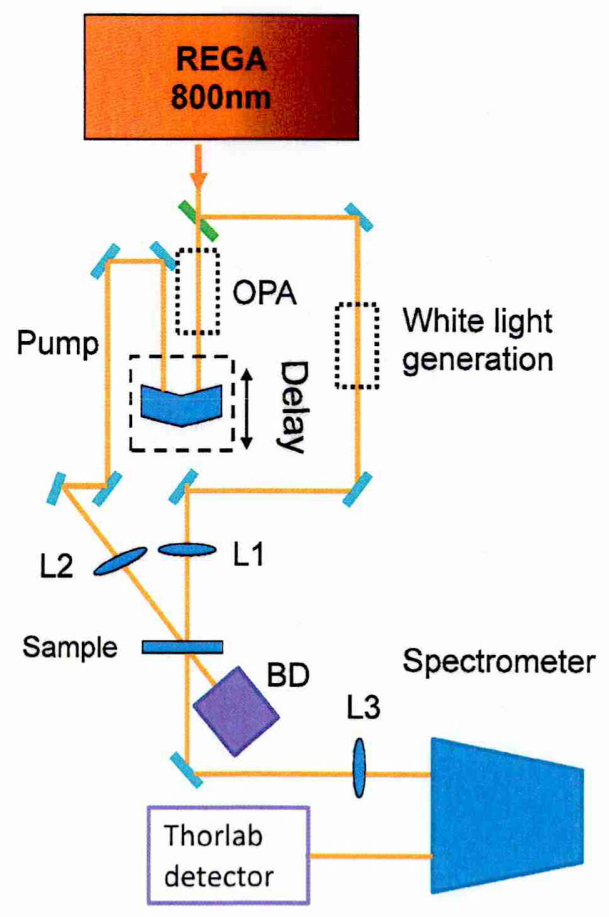

Figure 3-9: The experimental arrangements of spectrally resolved pump probe measurements. OPA = Optical Parametric amplifier, L1, L2, L3 are lens 1, lens2, lens3 respectively, $B D$ is beam dump. 


\section{CHAPTER 4}

\section{SAMPLE DETAILS AND CHARACTERISATION}

\subsection{Introduction}

This chapter presents details of the samples and their characterisation by different techniques. The chapter is divided into four main sections. In the first section, we described the method by which isolation of SWNTs was achieved. The second section presents the linear optical absorption characteristics of the nanotubes where each peak observed will be assigned to a specific nanotubes species. The length and diameter measurements using Atomic force Microscopy (AFM) will be discussed in the third section of this chapter. The fourth section will discuss a Raman study in order to compare the defect concentration of different samples of isolated SWNTs. This defect study is very important in order to explain the pump probe results presented in chapter 6 . The final and last section of this chapter will describe X-ray photoelectron spectroscopy (XPS) of the SWNTs. 


\subsection{Production of SWNTs Sample}

\subsubsection{Introduction}

Isolated SWNT samples are required to enable a clear picture of the photophysics as intertube interactions in bundled nanotubes homogenously broaden the spectra. Such broadening makes it difficult to distinguish the constituent nanotubes species and also introduces additional relaxation pathways for the excitons which makes the pump probe analysis more difficult. In this section, we will discuss the procedure through which these isolated samples were achieved.

\subsection{2 ss-DNA Wrapped HiPco SWNTS in Aqueous Suspension}

In 1999 R. E. Smalley et al. [21] developed a high pressure carbon monoxide method called 'HiPco' for the production of SWNTs. In this process, carbon monoxide acts as a feedstock and iron carbon monoxide $\mathrm{Fe}(\mathrm{CO})_{5}$ as a catalyst. Thermal decomposition of $\mathrm{Fe}(\mathrm{CO})_{5}$ produces iron atoms which coalesce to form metal clusters in a gas phase. These clusters continue to grow until they reach a diameter $(0.7-1.4 \mathrm{~nm})$ comparable to the diameter of SWNTs. After this, clusters of iron atoms act as catalyst to produce the solid carbon from carbon monoxide i.e.

$$
\mathrm{CO}+\mathrm{CO} \rightarrow \mathrm{C}(\mathrm{s})+\mathrm{CO}_{2}
$$

The SWNTs are produced from this solid carbon. A hemi-fullerene cap is formed on the surface of cluster which lifts up the cluster, and additional carbon atoms are attached at the edge of the cap. Thus forms a constant diameter hollow tube (SWNTs). With this method, SWNTs of high quality and with few structural defects can be produced. Through the optimisation of the growth temperature and pressure, HiPco nanotubes with diameter (0.7-1.2 nm) can be produced whose $E_{11}$ transitions are in the range of wavelengths which can be produced from our laser system.

Single-strand DNA wrapped HiPco SWNT samples used in our experiments were provided by our collaborator at the University of Southampton, UK. The detailed description of the sample preparation and characterisation can be found in ref [28]. SWNT-DNA hybrids 
were prepared by adding $3 \mathrm{mg}$ of HiPco SWNTs in aqueous solution of ss-DNA $(1 \mathrm{mg} / 1.5 \mathrm{ml})$ and dispersing them using high-shear sonication in an ice water bath at 45 Watt. After sonication, the samples were centrifuged at $12000 \mathrm{~g} \mathrm{~min}^{-1}$ for $90 \mathrm{~min}$. Then samples, containing the SWNT-DNA hybrids, were collected from the top.

\subsubsection{CoMoCat SWNTs in Aqueous Suspension}

In 2000, another method was proposed for the production of high quality SWNTs called 'CoMoCat' named after the unique catalyst mixture of cobalt and molybdenum [94]. In this method, the SWNTs are grown by the decomposition of carbon monoxide into simple carbon and carbon dioxide at the temperature of $700^{\circ} \mathrm{C}-950^{\circ} \mathrm{C}$ and pressure of $1-10 \mathrm{~atm}$. The CoMoCat SWNT samples used in our experiments were prepared by D. Mersch from the Physics department, University of Surrey. The detail description and initial characterisation of these samples can be found in ref [26].

\subsubsection{CoMoCat1 Sample}

The CoMoCat nanotubes with $50 \%$ of $(7,6)$ chirality, $>80 \%$ purity and $0.7-1.1 \mathrm{~nm}$ diameter were selected for the dispersion and sodium cholate (SC) was chosen as a surfactant. The $15 \mathrm{~g}$ of SWNT powder was mixed with $15 \mathrm{ml}$ of deionised water (DI water) and $2 \%$ weight/volume (w/v) of SC. The resultant dispersion was bath sonicated for $2 \mathrm{hr}$ in order to break the strong Van der Waals bonding of the nanotubes and after that it was left for $24 \mathrm{hr}$, so that nanotubes which are not dispersed yet could aggregate with SC [95]. Again the dispersion was bath sonicated for $1 \mathrm{hr}$ and left it for another $24 \mathrm{hr}$. After waiting for $24 \mathrm{hr}$, the dispersion was bath sonicated for another $1 \mathrm{hr}$ to break the remaining bundles of carbon nanotubes in the dispersion. The bath sonication produced a uniform dispersion of the SWNTs. This uniform dispersion was centrifuged at $64000 \mathrm{rpm}$ or $17400 \mathrm{~g}$ for 14 minutes to filter out impurities. After the centrifugation, the top $80 \%$ was taken for our experiments.

\subsubsection{CoMoCat2 Sample}

The separation of CoMoCat2 SWNT sample was done using the density gradient ultracentrifugation (DGU), a powerful tool widely used in separation of SWNTs by property like diameter or electrical type. In our case, separation was achieved by electric properties 
using DGU. The detailed procedure and initial characterisation using Raman spectroscopy and UV-Vis spectroscopy can be found in ref [26]. In this method, two surfactants (SC and SDS) were used with a ratio SDS:SC $=1: 4$ but the total percentage of all the surfactants in the solution was $2 \% \mathrm{w} / \mathrm{v}$. The density gradient was built linearly from $15 \%$ to $30 \%$ and $60 \%$ layer using $60 \%$ solution of iodixanol and ultracentrifugation was done at the speed of $45000 \mathrm{rpm}(86000 \mathrm{~g})$ for 14 hours. After the separation top $20 \%$ was taken for the measurements.

\subsubsection{Structured CNT-Polymer Composite}

Exploratory measurements were performed on stretch-aligned SWNT-polymer samples. These samples were prepared by I. Jurewicz and Dr. Alan Dalton in the Physics department, University of Surrey. The detailed fabrication procedure is described in detail in [96]. The non-ionic Triton X-100 was used as a surfactant to disperse the bundle of SWNTs in aqueous solution.

A suspension of nanotubes was prepared by adding $50 \mathrm{mg}$ of nanotubes to 50 gram of water surfactant solution and then sonicating it in an ice water bath using a probe-tip sonicator at 20 watts for 20 minutes in order to break the large cluster of nanotubes. After this, 5 grams of latex was combined with 12.375 grams of the aqueous dispersion of nanotubes before sonicating them in both continuous and discontinuous mode for 20 minutes. After the sonication, the mixture was pipetted into a PTFE trough and left for 24 hours to dry. Once it was completely dried, it was cut into strips of various dimensions. The samples series were obtained by stretching each sample stripe to various percentage (\%) increase of its original length and each sample was secured onto a glass substrate by using doubled-sided tape.

\subsection{Linear Optical Characterisation}

There exist singularities in the electronic density of states (DOS) of SWNTs due to its one dimensional nature (as discussed in chapter 2). This pseudo one dimensional nature of SWNTs can be probed by optical absorption spectroscopy, a proven technique used to probe one dimensional character previously, and an inverse relationship between diameter and interband transition energy for individual nanotubes has been demonstrated using this 
technique [97]. This section will describe the linear absorption spectra taken on ss-DNA wrapped HiPco, CoMoCat1, CoMoCat2 SWNTs, and stretch-aligned SWNT-polymer samples. HiPco and CoMoCat samples were optically dense and homogenous, and we found them remarkably stable especially DNA wrapped SWNTs after long period of time. In the case of DNA wrapped sample, no changes were found in the linear absorption spectra over period of $>$ three years.

A Cary 5000-UV-Vis-IR spectrophotometer was used to obtain the linear absorption spectra of SWNT samples in the wavelength range of 300-3300 nm using a range of detectors and sources [98]. A Spectrosil ${ }^{\mathrm{TM}}$ fused quartz cuvette (1 mm path length) was selected to hold the SWNT suspension for these measurements because of its purity and low absorption in the wavelength range of interest.

\subsubsection{Absorption Measurement of ss-DNA HiPco SWNTs}

The absorption spectrum of ss-DNA wrapped HiPco SWNTs is shown in Fig. 4-1. The spectrum is characterised by many narrow peaks due to interband transitions in both semiconducting and metallic nanotubes. The presence of these individual sharp peaks in the absorption spectrum indicates that SWNTs in our samples are well isolated. However a large background is observed in the absorption spectrum which has been attributed to a $\pi$-plasmon resonance centred at $5 \mathrm{eV}[81,82]$. This probably originates due to $\mathrm{C}_{60}$ fullerene, graphitic carbon, metallic nanotubes, and some other residual carboneous impurities [83]. This background recently has been completely removed from the linear absorption spectrum of carbon nanotubes purified in a process using PFO [88].

In order to assign each peak to specific nanotubes species present in our sample, the exact position of the peak must be accurately known. The position can be determined through the fitting of absorption spectrum with multiple set of Lorentzian functions. The presence of a large background is the main source of unreliability in such a fitting. If the linear absorption spectrum is plotted as function of energy, an approximately linear slop is recognised which can be easily subtracted. So we fitted the background-free absorption spectrum with multiple Lorentzians. We introduced a global shift parameter ' $\Delta E_{c}$ ' to account the environmental effect on transition energies. 


$$
f(E)=\alpha_{0}+\sum_{i} \frac{2 A_{i}}{\pi} \frac{w_{i}}{4\left(E-E_{c, i}-\Delta E_{c}\right)^{2}+w_{i}^{2}} .
$$

where $\alpha_{0}$ is constant background (offset), whose value was set to zero initially, $A_{i}$ is the amplitude due to species $\mathrm{i}, E_{c, i}$ is the transition energy of species i, $w_{i}$ is FWHM of Lorentzians, and $\Delta E_{c}$ is global shift in the peak position. The background free absorption spectrum fitted with multiple Lorentzians is shown in Fig. 4-2. The energies of Lorentzians were fixed to the experimentally observed values, but the widths and amplitudes were allowed to vary.

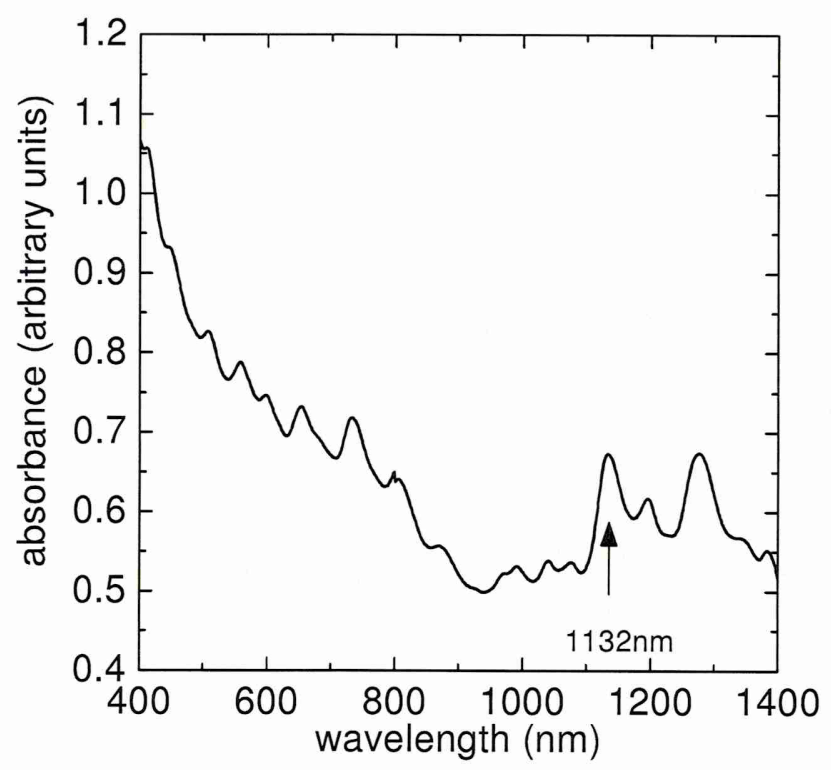

Figure 4-1: Absorption spectrum of ss-DNA wrapped HiPco SWNTs. The arrow corresponds to wavelength selected for the pump probe measurements presented in chapter 5 of this thesis.

The absorption spectrum in Fig. 4-2 is plotted from $\sim 0.85 \mathrm{eV}$ to $2.5 \mathrm{eV}$ which covers the $E_{11}$ and $E_{22}$ transitions of semiconducting nanotubes as $E_{11}$ transition lies between $0.85-\sim 1.4 \mathrm{eV}, E_{22}$ transitions lies between $1.4-\sim 2.6 \mathrm{eV}$ and higher energy peaks are attributed to metallic nanotubes for the HiPco nanotubes with diameter mostly centred between $0.7-1.2 \mathrm{~nm}[21]$ and are not included in our discussion.

The species in our sample are identified through a comparison of peaks observed in the 
background free absorption spectra shown in Fig. 4-2 with the tabulated energies given in [99]. The number of nanotubes species assigned to each peak is more than one due to the close spacing of $(n, m)$ species [99]. Therefore we restrict ourselves to potential nanotubes species by comparing the absorption spectrum in the region of $E_{11}$ and $E_{22}$ transitions of absorption spectrum (Fig. 4-2). If the species is present in each sub band, then it is included in the table 4-1. Another important point is global energy shift of $\Delta E_{c}=-(4 \pm 1) \mathrm{meV}$ as compared to the tabulated value given in ref [99]. This may be related to different environment of SWNTs in our sample as compared to those used in ref [99]. Similar effect (energy shift) was found when using ammonium cholate suspension as compared to the SDSassisted suspension [100].

We note that photoluminescence excitation is a preferred method to classify nanotube species since it simultaneously identifies the $E_{11}$ and $E_{22}$ transitions; however we did not have access to this technique.

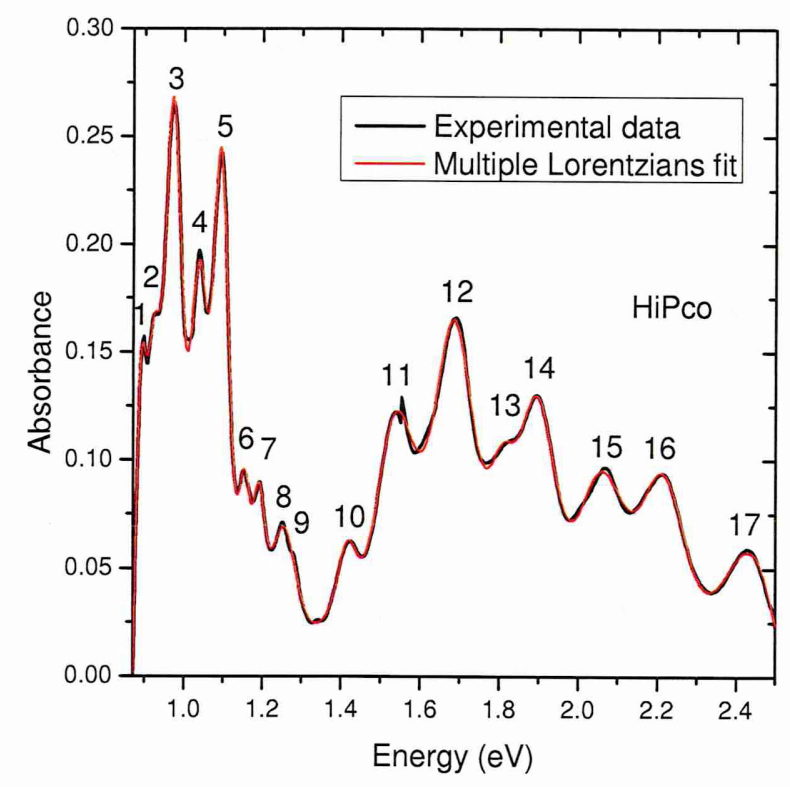

Figure 4-2: Background-subtracted absorption spectra of ss-DNA wrapped HiPco SWNTs along with a fit using multiple Lorentzians. Each peak is labelled corresponding to the specific nanotube species indicated in Table 4.1.

The wavelength of $1132 \mathrm{~nm}$ which corresponds to an energy of $\sim 1.1 \mathrm{eV}$ is selected for the degenerate pump probe measurements in case of ss-DNA wrapped HiPco SWNTs which contains possible contributions from $(7,6),(9,2)$, and $(8,4)$ with diameters $0.88 \mathrm{~nm}, 0.79 \mathrm{~nm}$, 
and $0.83 \mathrm{~nm}$ respectively. The main advantages of selecting this wavelength are: (1) it is a prominent peak in the HiPco spectrum, (2) it is also a prominent peak in the CoMoCat spectrum described in 4.3.2, facilitating comparison of the samples, and (3) it can easily be generated from our optical parametric amplifier (OPA) and sufficient pump power can be generated to probe the saturation of exciton density on the nanotubes.

However the spectral width of peak selected for pump probe measurement is $\sim 60 \mathrm{meV}$ which is much higher than previously reported value of $8-20 \mathrm{meV}$ [47, 101-103] using photoluminescence (PL) spectroscopy and four wave mixing technique (FWM). This large value of spectral width shows that transitions in our samples are inhomogenously broadened.

\begin{tabular}{|l|l|l|l|l|l|l|}
\hline $\begin{array}{l}\text { Chirality } \\
(\mathrm{n}, \mathrm{m})\end{array}$ & $\mathrm{E}_{11}$ peak & $\begin{array}{l}\mathrm{E}_{11} \\
(\mathrm{eV})\end{array}$ & $\begin{array}{c}\mathrm{E}_{11} \text { width } \\
(\mathrm{eV})\end{array}$ & $\begin{array}{c}\mathrm{E}_{22} \\
(\mathrm{eV})\end{array}$ & $\mathrm{E}_{22}$ peak & $\begin{array}{c}\mathrm{E}_{22} \text { width } \\
(\mathrm{eV})\end{array}$ \\
\hline$(9,8)$ & 1 & 0.879 & 0.043 & 1.533 & 11 & 0.182 \\
$(10,8)$ & 1 & 0.844 & & 1.426 & 10 & 0.096 \\
\hline$(12,2)$ & 2 & 0.900 & 0.055 & 1.807 & 13 & 0.118 \\
\hline$(8,7)$ & 3 & 0.981 & 0.067 & 1.702 & 12 & 0.167 \\
$(11,1)$ & 3 & 0.980 & & 2.032 & 15 & 0.220 \\
\hline$(11,3)$ & 4 & 1.036 & 0.066 & 1.564 & 11 & 0.182 \\
\hline$(7,6)$ & 5 & 1.107 & 0.061 & 1.914 & 14 & 0.156 \\
$(9,2)$ & 5 & 1.092 & & 2.251 & 16 & 0.203 \\
$(8,4)$ & 5 & 1.116 & & 2.105 & 15 & 0.220 \\
\hline$(10,2)$ & 6 & 1.177 & 0.050 & 1.683 & 12 & 0.169 \\
$(9,4)$ & 6 & 1.126 & & 1.716 & 12 & \\
\hline$(7,5)$ & 7 & 1.211 & 0.049 & 1.921 & 14 & 0.156 \\
$(11,0)$ & 7 & 1.196 & & 1.665 & 12 & 0.169 \\
\hline$(7,3)$ & 8 & 1.250 & 0.132 & 2.457 & 17 & 0.336 \\
$(6,5)$ & 8 & 1.27 & & 2.19 & 16 & 0.203 \\
\hline$(9,1)$ & 9 & 1.359 & 0.160 & 1.794 & 13 & 0.118 \\
\hline
\end{tabular}

Table 4-1: The $(n, m)$ species present in aqueous solution of ss-DNA wrapped HiPco SWNTs along with $1^{\text {st }}$ and $2^{\text {nd }}$ transitions, width of Lorentzian peaks used in the fitting, and corresponding peak position in the absorption spectrum. 


\subsubsection{Absorption Measurement of CoMoCat1 SWNT Sample}

The linear absorption spectrum of CoMoCat1 SWNTs is shown in Fig. 4-3. The spectrum is qualitatively similar to the absorption spectrum of ss-DNA wrapped HiPco nanotubes i.e. there are clearly distinguished narrow peaks due to interband transitions superimposed on the $\pi$-plasmon background but with fewer distinct species of nanotubes in the $E_{11}$ region. The presence of narrow isolated peaks in the absorption spectrum again indicates that the nanotubes are well isolated.

Five main features dominate in the $\mathrm{E}_{11}$ region of the absorption spectrum of CoMoCat1 SWNTs as shown in Fig. 4-3 of which peak 2 is the most prominent in the $E_{11}$ region whereas peak 8 is dominant in the $E_{22}$ region. These are $E_{11}$ and $E_{22}$ transitions of $(7,6)$ nanotubes and are dominant because the untreated SWNT powder contains $50 \%$ of $(7,6)$ nanotube. The wavelength of $1142 \mathrm{~nm}$ is selected for pump probe measurements (discussed in chapter 6) which means that $(7,6)$ SWNTs are selected.

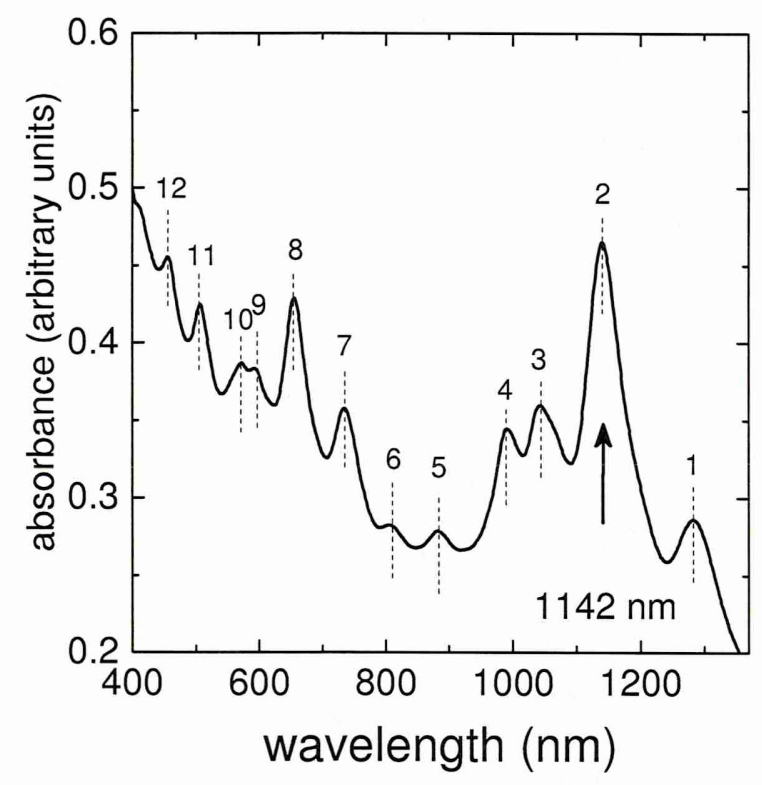

Figure 4-3: Absorption spectrum of CoMoCat1 SWNTs. The numbers label the transitions which arise from interband transitions in both metallic and semiconducting nanotubes.

Figure 4-3 shows that there is less background contribution due to $\pi$-plasmon resonance as compared to ss-DNA wrapped HiPco nanotubes which may be due to fewer metallic species present in the CoMoCat1 SWNT sample. The background-free absorption spectrum fitted with set of Lorentzians using equation 4.2 is shown in Fig. 4-4. 


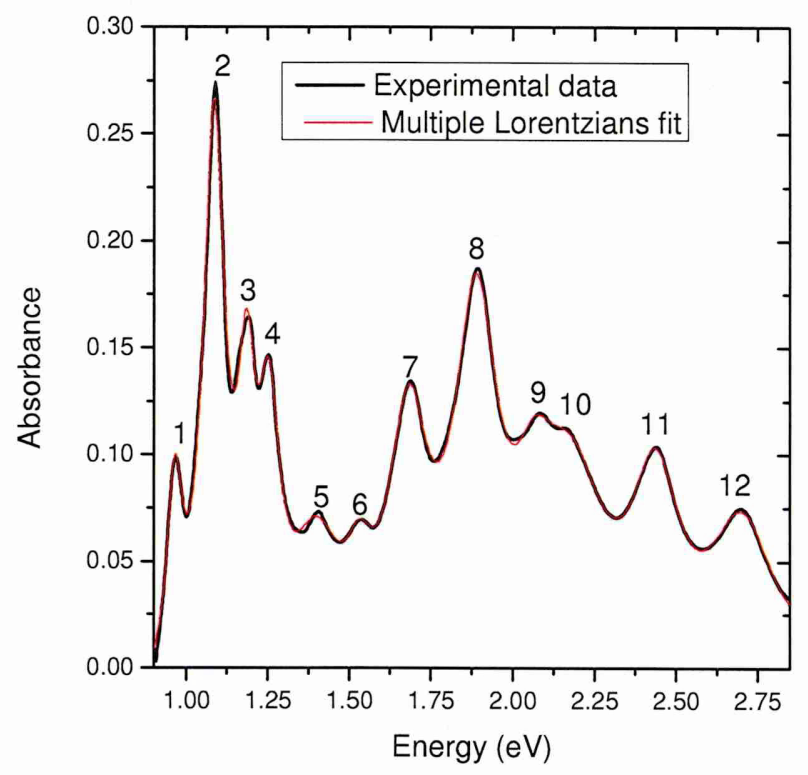

Figure 4-4: Background free absorption spectrum of CoMoCat1 SWNTs as function of energy (eV) fitted with Lorentzians.

\begin{tabular}{|c|c|c|c|c|c|c|}
\hline $\begin{array}{c}\text { Chirality } \\
(\mathrm{n}, \mathrm{m})\end{array}$ & $\mathrm{E}_{11}$ peak & $\begin{array}{c}\mathrm{E}_{11} \\
(\mathrm{eV})\end{array}$ & $\begin{array}{c}\mathrm{E}_{11} \text { width } \\
(\mathrm{eV})\end{array}$ & $\begin{array}{c}\mathrm{E}_{22} \\
(\mathrm{eV})\end{array}$ & $\mathrm{E}_{22}$ peak & $\begin{array}{c}\mathrm{E}_{22} \text { width } \\
(\mathrm{eV})\end{array}$ \\
\hline$(9,7)$ & 1 & 0.938 & 0.058 & 1.563 & 6 & 0.103 \\
$(11,1)$ & 1 & 0.980 & & 2.032 & 9 & 0.147 \\
\hline$(7,6)$ & 2 & 1.107 & 0.082 & 1.914 & 8 & 0.163 \\
\hline$(10,2)$ & 3 & 1.177 & 0.066 & 1.683 & 7 & 0.149 \\
\hline$(7,3)$ & 4 & 1.250 & 0.081 & 2.457 & 11 & 0.187 \\
$(6,5)$ & 4 & 1.270 & & 2.190 & 10 & 0.268 \\
\hline$(6,4)$ & 5 & 1.42 & 0.191 & 2.146 & 10 & 0.268 \\
\hline
\end{tabular}

Table 4-2: The $(n, m)$ species present in aqueous solution of CoMoCat1 SWNTs along with $1^{\text {st }}$ and $2^{\text {nd }}$ transitions, width of Lorentzian peaks used in the fitting, and corresponding peak position in the absorption spectrum shown in Fig. 4-4.

The energy shift of $\Delta E_{c}=-(2 \pm 1) \mathrm{meV}$ was found as compared to the tabulated value given in [99]. The spectrum is plotted from $0.9 \mathrm{eV}$ to $3.0 \mathrm{eV}$ which covers the whole range of 
$E_{11}$ and $E_{22}$ transitions of semiconducting nanotubes. The possible nanotube species contributing to each peak is given in table 4-2. The spectral width of peak selected for pump probe measurement is $\sim 82 \mathrm{meV}$, a higher value than HiPco SWNTs.

\subsubsection{CoMoCat2 Sample}

The absorption spectrum of CoMoCat2 sample is shown in Fig. 4-5. There are fewer peaks in the $\mathrm{E}_{11}$ region as compared to HiPco and CoMoCat1 sample because this sample is purified for a single species $(6,5)$. After the separation, the sample contains $57 \%$ of $(6,5)$ which has $E_{11}$ transition at $992 \mathrm{~nm}$ and $E_{22}$ transition at $576 \mathrm{~nm}$ [99]. These two peaks are the dominant features observed in the absorption spectrum of sample as shown in Fig. 4-5. However the features in the $E_{11}$ region of absorption spectrum are broader, suggesting possible bundling of the nanotubes in the sample. The pump probe measurements of this sample will be presented in chapter 6 .
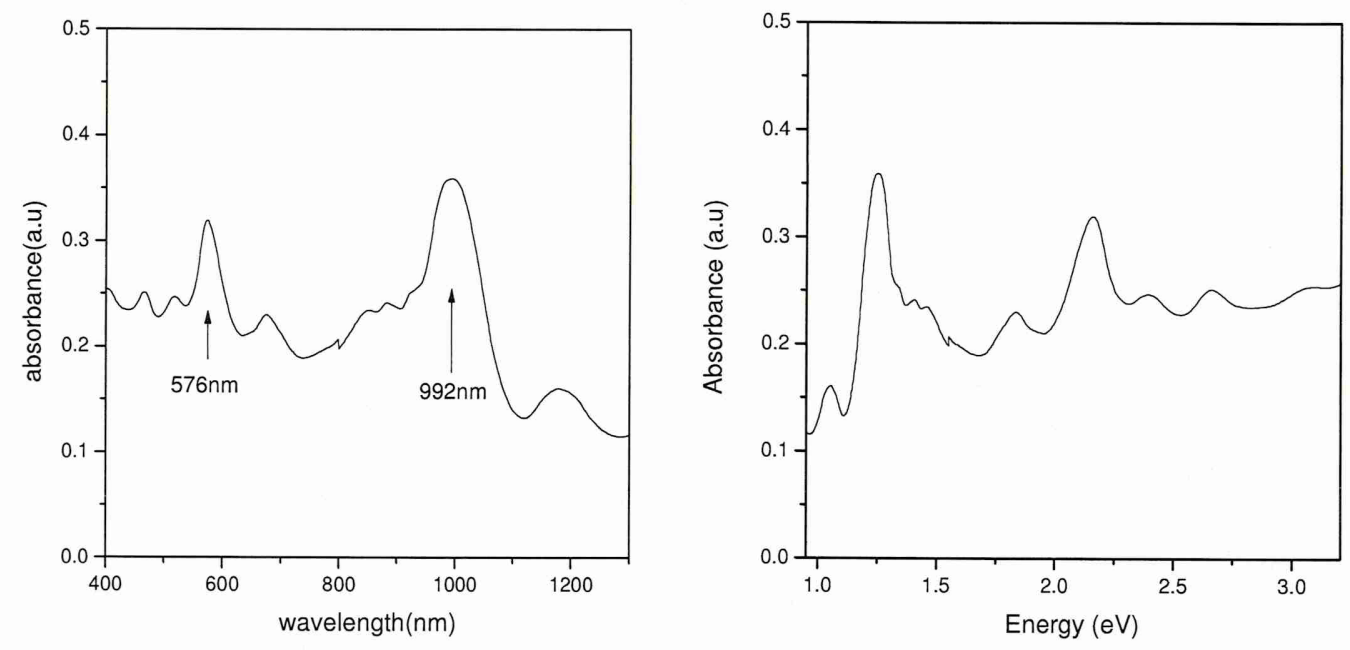

Figure 4-5: Absorption spectrum of CoMoCat2 Sample (a) plotted as function of wavelength (b) as function of energy. The features in the $E_{11}$ region are broader as compared to other samples discussed above, suggesting a greater degree of bundling.

\subsubsection{Structured CNT-Polymer Composite}

The absorption spectrum of the SWNT-polymer composite described in section 4.2.4 is shown in Fig. 4-6. The peaks in the absorption spectrum are quite broad, indicating 
inhomogeneous broadening possibly due to bundling of the nanotubes. We selected a wavelength of $1170 \mathrm{~nm}$ for our pump probe measurement because the sample has strong absorption at this wavelength (see Fig. 4-6). At this wavelength, we are expecting a contribution from the following species: $(8,6),(10,0)$, and $(11,3)[99]$.
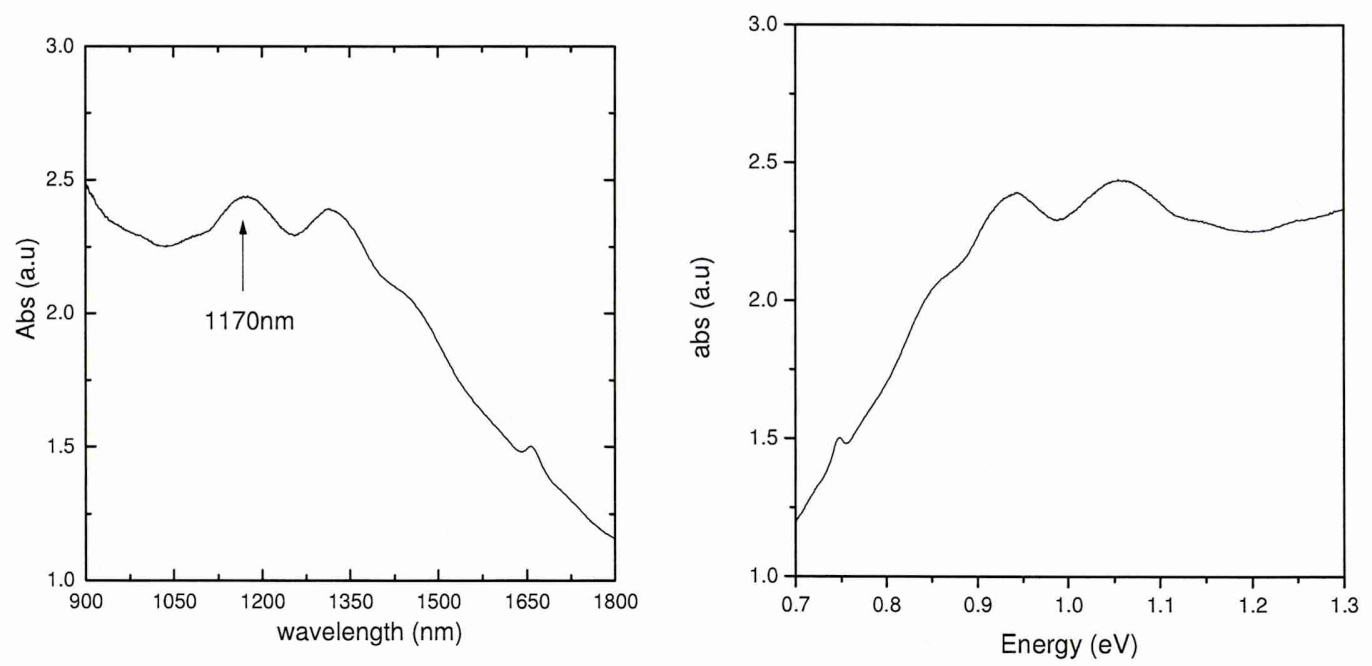

Figure 4-6: Absorption spectrum of SWNT-polymer composite in latex film (a) plotted as a function of wavelength (b) as function of energy.

\subsection{Length and Diameter Distribution Study of SWNTs by Atomic Force Microscopy (AFM) Measurements}

A comprehensive AFM study was performed to measure the tube diameter and tube length of DNA wrapped HiPco and CoMoCat SWNTs. I am thankful to Dr. Cristina Giusca and Dr. Izabella Jurewicz for these measurements.

\subsubsection{DNA wrapped HiPco SWNTs}

This section describes the AFM measurements on ss-DNA wrapped HiPco SWNTs. The sample was prepared by placing a drop of solution onto a mica substrate and drying it at room temperature. The AFM images were taken by employing the Atomic Force Microscope in semi contact mode. The AFM images of DNA wrapped HiPco SWNTs are shown in Fig. 4-7. The Fig. 4-7 (a) shows the height profile image which was used to measure the length of 
nanotubes. The image in Fig. 4-7 (b) was obtained employing the phase mode which is used to measure the chemical nature of the material. The phase will change in the presence of impurities or surfactant etc in the sample. As shown in Fig. 4-7 (b), there are short sections of nanotube of typical length $\sim 200 \mathrm{~nm}$. Also there are striations visible on the tubes which are evidence of DNA wrapping. The large agglomerates are most likely due to impurities in the raw nanotube sample or free DNA molecules.

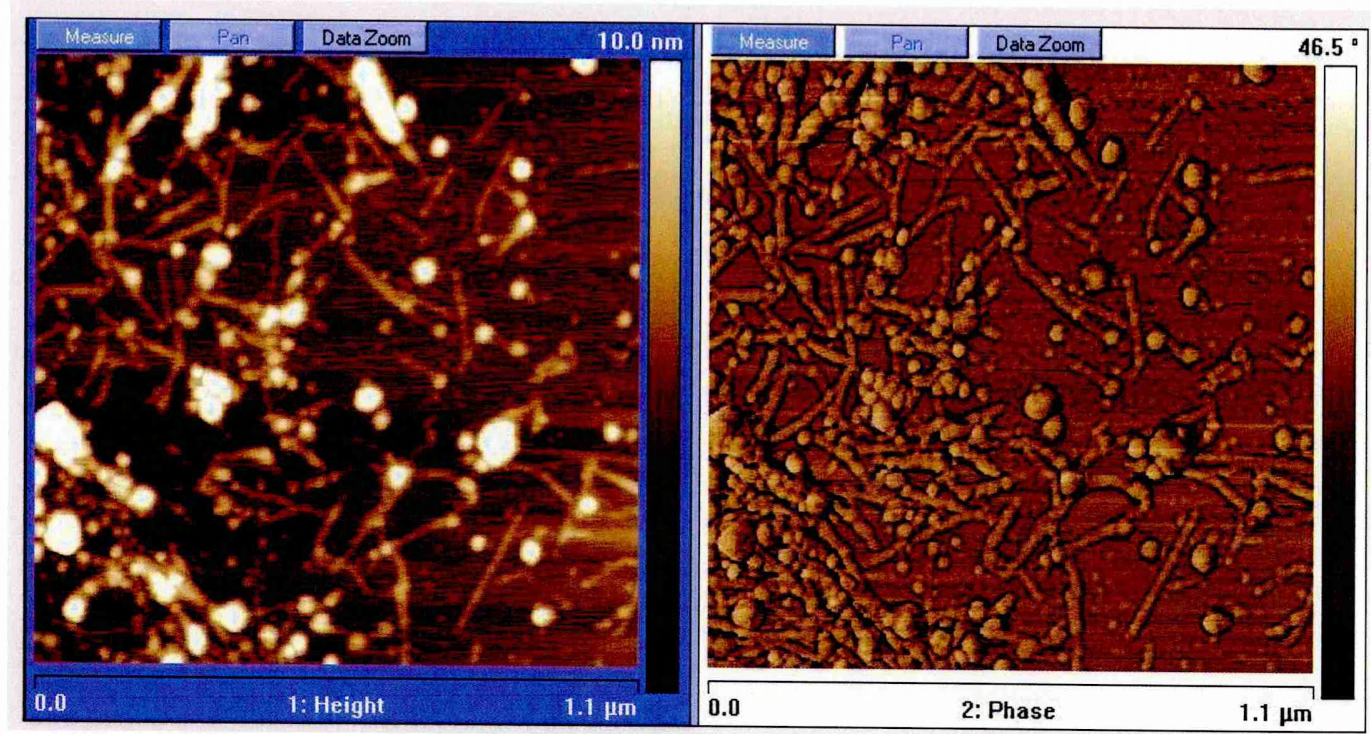

Figure 4-7: AFM image of ss-DNA wrapped HiPco SWNTs. (a) AFM height profile image used to measure the length of nanotubes. (b) Phase mode used to identify the wrapping of DNA around the nanotube.
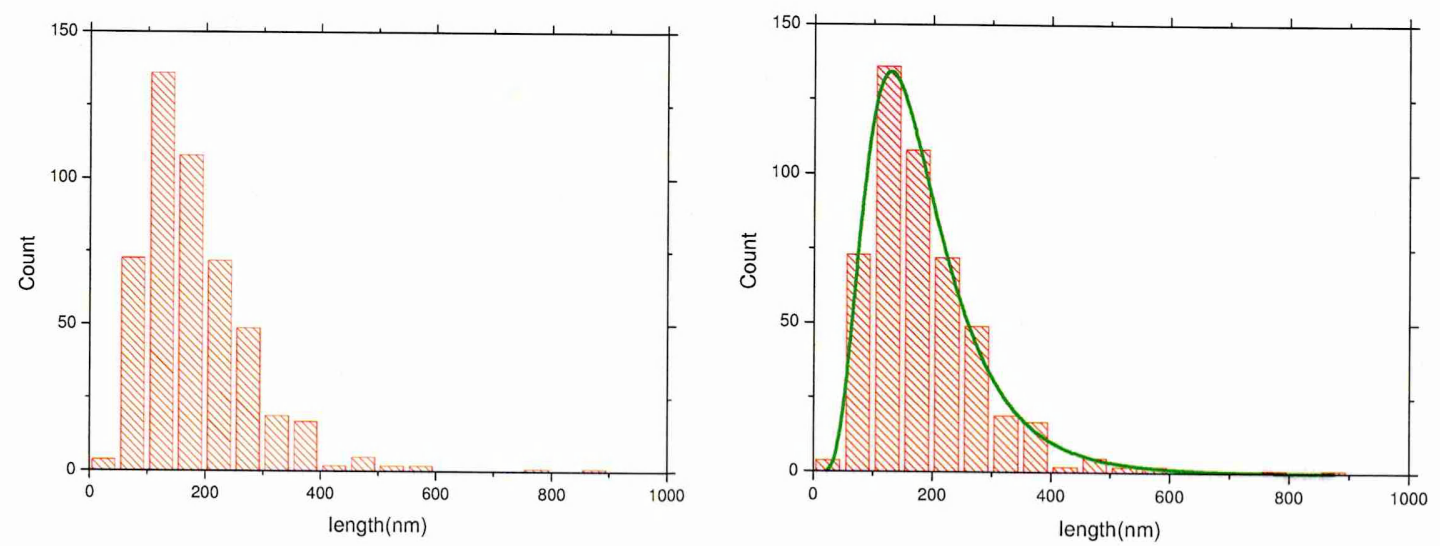

Figure 4-8: (a) Nanotube length histograms of ss-DNA wrapped HiPco SWNTs obtained from the AFM images shown in Fig. 4-7. (b) Nanotube length histograms fitted with LogNormal distribution. 
Length distribution of 491 randomly selected nanotubes is shown in Fig. 4-8. The measured average length $(184 \pm 5) \mathrm{nm}$ is short as compared to the as-grown HiPco nanotubes, where a length of $\sim 1 \mu \mathrm{m}$ is expected. This is due to the helical wrapping of the DNA around the nanotubes which cut the nanotubes significantly [104]. The shorter nanotubes are very important to observe the single exciton regime in the dynamical measurements of SWNTs which will be discussed in detail in chapter 5 .

\subsubsection{CoMoCat1 Sample}

The CoMoCat1 sample for AFM study was prepared by taking the CoMoCat1 SWNTs suspension from our stock solution and spin coating it onto a mica substrate at a speed of $4000 \mathrm{rpm}$ for $20 \mathrm{sec}$ and drying it in air for $15 \mathrm{~min}$ at room temperature. The diameter and length of 52 separated SWNTs was determined using the AFM height profile. The resultant diameter and length distribution of CoMoCat1 SWNTs are plotted in Fig. 4-9.
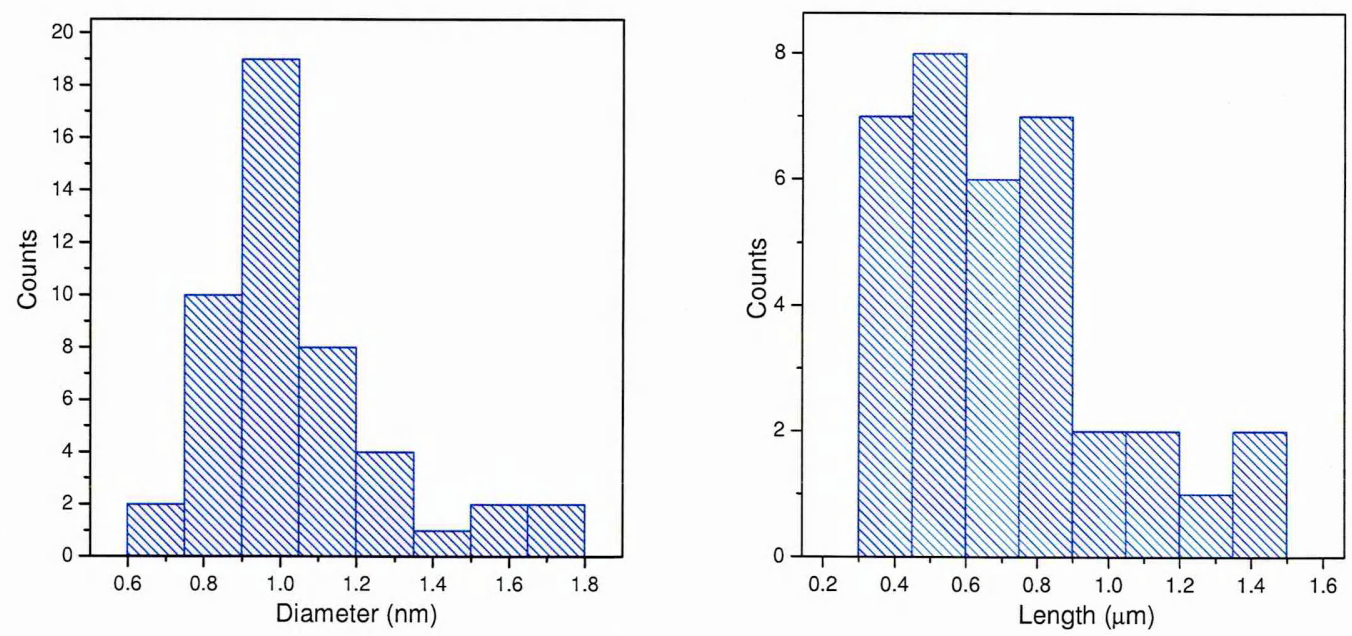

Figure 4-9: AFM measurement of CoMoCat1 sample (a) diameter distribution (b) length distribution measured using the height profile of AFM images. The total number of SWNTs measured was 52.

Average nanotubes diameter measured is $(1.05 \pm 0.04) \mathrm{nm}$ which is in good agreement with reported value of $(0.75-1.22 \mathrm{~nm})$ in the literature for CoMoCat SWNTs. However, the measured average diameter is larger as compare to the diameter $(\sim 0.9 \mathrm{~nm})$ of $(7,6)$ nanotube and it may be due to presence of surfactant. The average length measured is 
$(766 \pm 48) \mathrm{nm}$ which is longer than ss-DNA wrapped HiPco nanotubes.

\subsubsection{CoMoCat2 Sample}

CoMoCat2 SWNTs dispersions taken from stock solution of sample were spin-coated (at $4000 \mathrm{rpm}$ for $20 \mathrm{sec}$ ) onto a mica substrate followed by drying it in the air for $15 \mathrm{~min}$. The AFM measurements were done using the semi-contact mode. AFM height images of separated SWNTs are shown in Fig. 4-10.

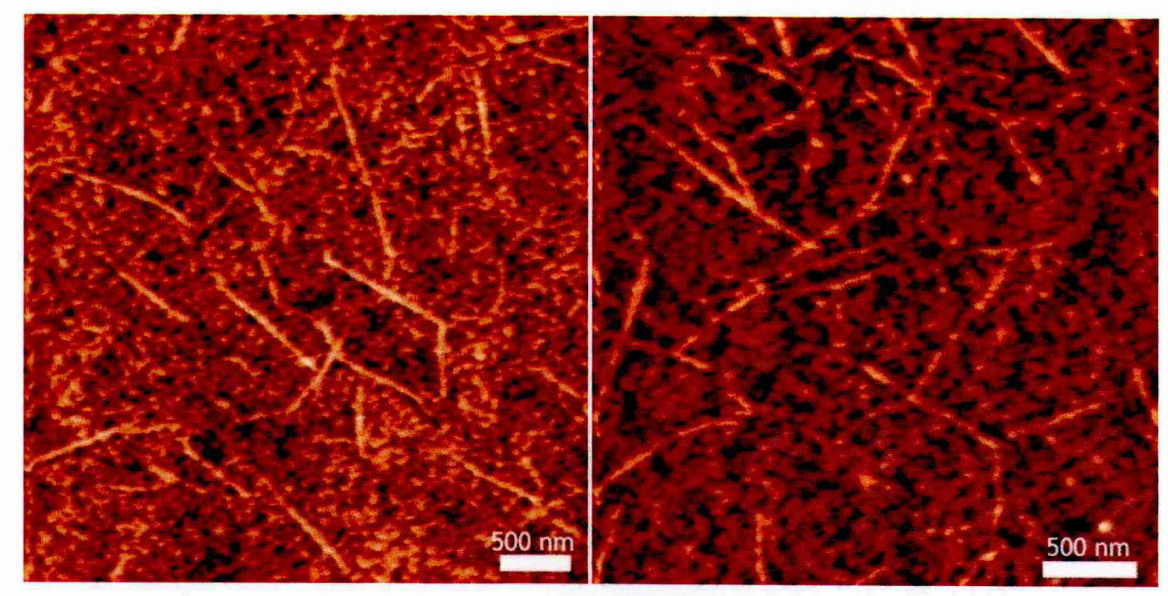

Figure 4-10: AFM images of CoMoCat2 SWNTs sample. The SWNTs in this sample are longer than previous samples studied. Also AFM images show that nanotubes are not straight which gives rise to defect centres for the excitons in the pump probe measurements discussed in chapter 6.

From the height profile images shown in Fig. 4-10, individual isolated SWNTs are observed with some kinks which may give rise to potential fluctuations which act as barriers and block the movement of excitons along the nanotubes during the pump probe measurements. Details on the effect of defects on exciton transport will be discussed in chapter 6.

The diameter of separated SWNTs was determined using the AFM height profile along the line drawn across the image as shown in Fig. 4-11. The diameter and length distribution of 97 SWNTs is given in Fig. 4-12. The averaged measured diameter is $(2.08 \pm 0.04) \mathrm{nm}$ which is greater than expected value and may be due to presence of surfactant, and average length measured is $(877 \pm 35) \mathrm{nm}$ which is larger than both ss-DNA wrapped HiPco and 
CoMoCat1 SWNT sample.

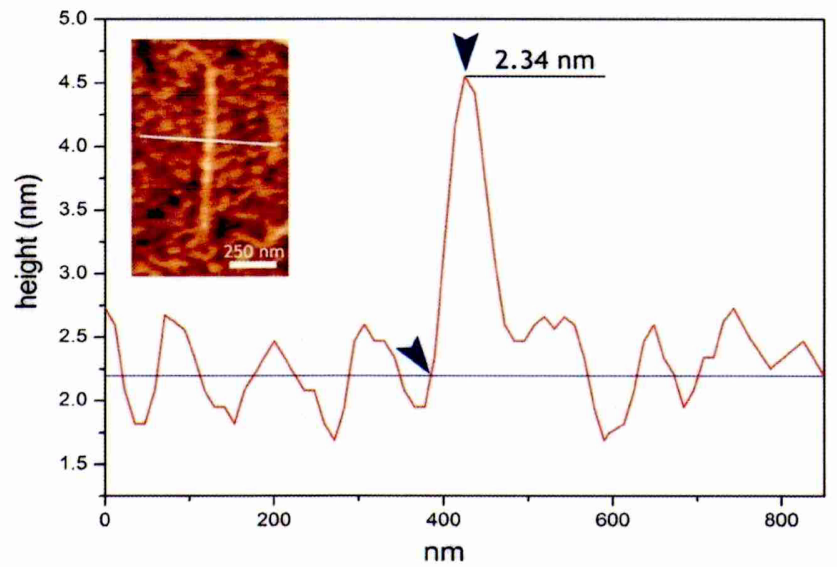

Figure 4-11: AFM height profile of CoMoCat2 SWNTs used to measure the diameter of nanotubes.
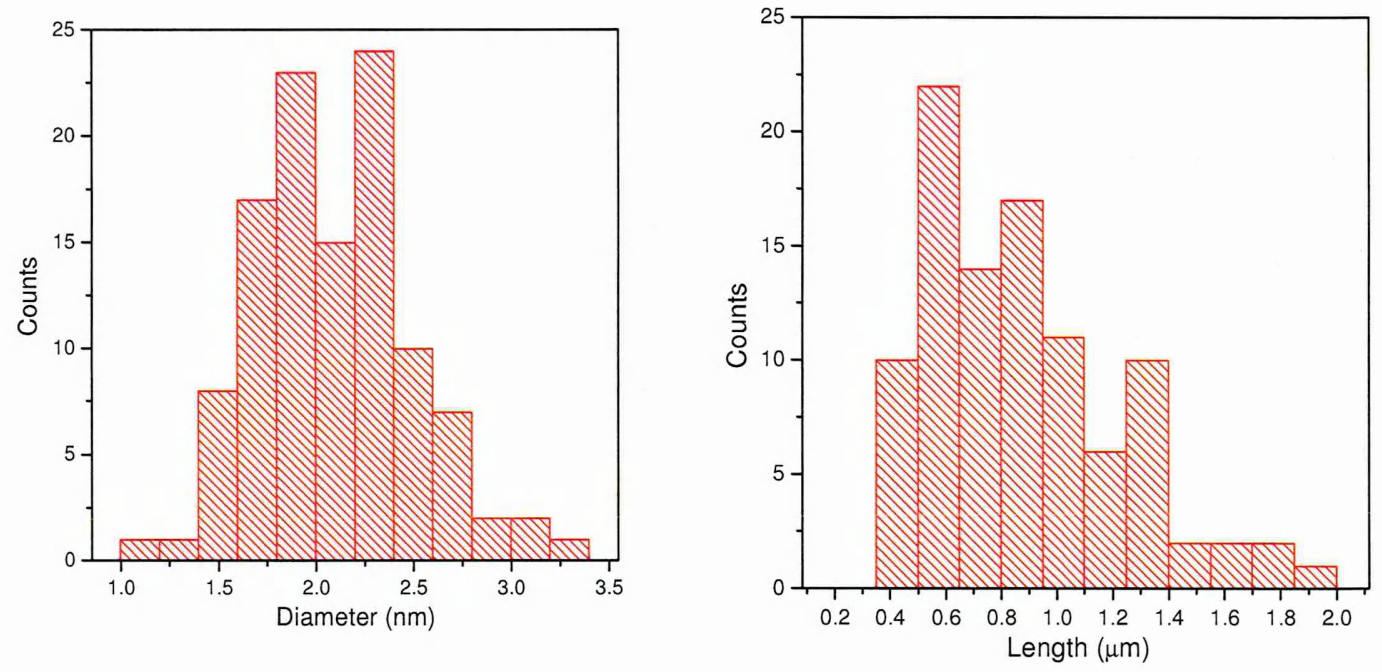

Figure 4-12: Diameter and length distribution of CoMoCat2 SWNTs sample, measured using the height profile of AFM images.

In conclusion, a comprehensive AFM study of SWNTs was carried out to measure the diameter and the length of nanotubes present in our samples. These measurements are important to discuss our pump probe results and also to measure the exciton size of the nanotubes which scales with diameter of the nanotubes. AFM results of nanotubes length, standard deviation, and standard error in mean are summarised in the table 4-3 


\begin{tabular}{|l|c|l|c|c|c|c|c|}
\hline Sample & $\begin{array}{c}\text { No. of SWNTs } \\
\text { measured }\end{array}$ & Mean & $\begin{array}{c}\text { Standard } \\
\text { Deviation }\end{array}$ & $\begin{array}{c}\text { Standard Error } \\
\text { in mean }\end{array}$ & Min length & Median & Max length \\
\hline $\begin{array}{l}\text { ss-DNA } \\
\text { HiPco }\end{array}$ & 491 & 184 & 100 & 5 & 43 & 163 & 890 \\
\hline CoMoCat1 & 52 & 766 & 346 & 48 & 327 & 696 & 1650 \\
\hline CoMoCat2 & 97 & 877 & 348 & 35 & 415 & 817 & 1912 \\
\hline
\end{tabular}

Table 4-3: Average nanotubes length, standard deviation, and standard error in mean calculated from AFM data.

\subsection{Raman Spectroscopy of Nanotubes}

The Raman spectroscopy of carbon nanotubes reveals information about 1D properties of material and their unusual electronic and phonon properties as well as about the imperfections (defects). Raman scattering is an inelastic process; during Raman scattering, the electron is excited from valence band to virtual higher states. This excited electron is subsequently scattered through emission (Stokes process) or absorption (anti-Stokes process) of a phonon and relaxes to valence band by emitting a photon. The Raman spectrum is observed for the scattered light (photon) with energy higher or lower than of the incident photon $[105,106]$.

In this section of the chapter, we present a Raman study of SWNTs and compare the results in terms of the defect concentration in two different samples (HiPco and CoMoCat). Raman spectroscopy was carried out with an excitation wavelength of $514 \mathrm{~nm}$ using microRaman Renishaw microscope. This section is divided into three parts. In the first part, we will discuss the Raman spectra of as-grown SWNT powder. The second section will compare the Raman spectra of purified dry SWNTs and Raman spectra of SWNTs in aqueous solution as well as comparison of defect density will be given in the third section.

\subsubsection{Raman Spectra of SWNT Powder}

Raman spectra of unprocessed HiPco and CoMoCat SWNT powder are plotted in Fig. 413. There are many dominant features which can be identified from Raman spectra of 
SWNTs includes the radial breathing mode (RBM), tangential mode ( $\mathrm{G}$ mode) and also second order Raman scattering processes including the defective mode (D mode) and its second harmonic ( $G^{\prime}$ mode). The details of these modes will be discussed later. In addition to these main features, there are many weak features such as iTOLA band (a combination of optical and acoustic modes), M band (overtone mode), and intermediate frequency modes (IFM) which can be assigned to one- or two-phonon double resonance Raman process. Details can be seen in $[105,106]$ and will not be discussed here.
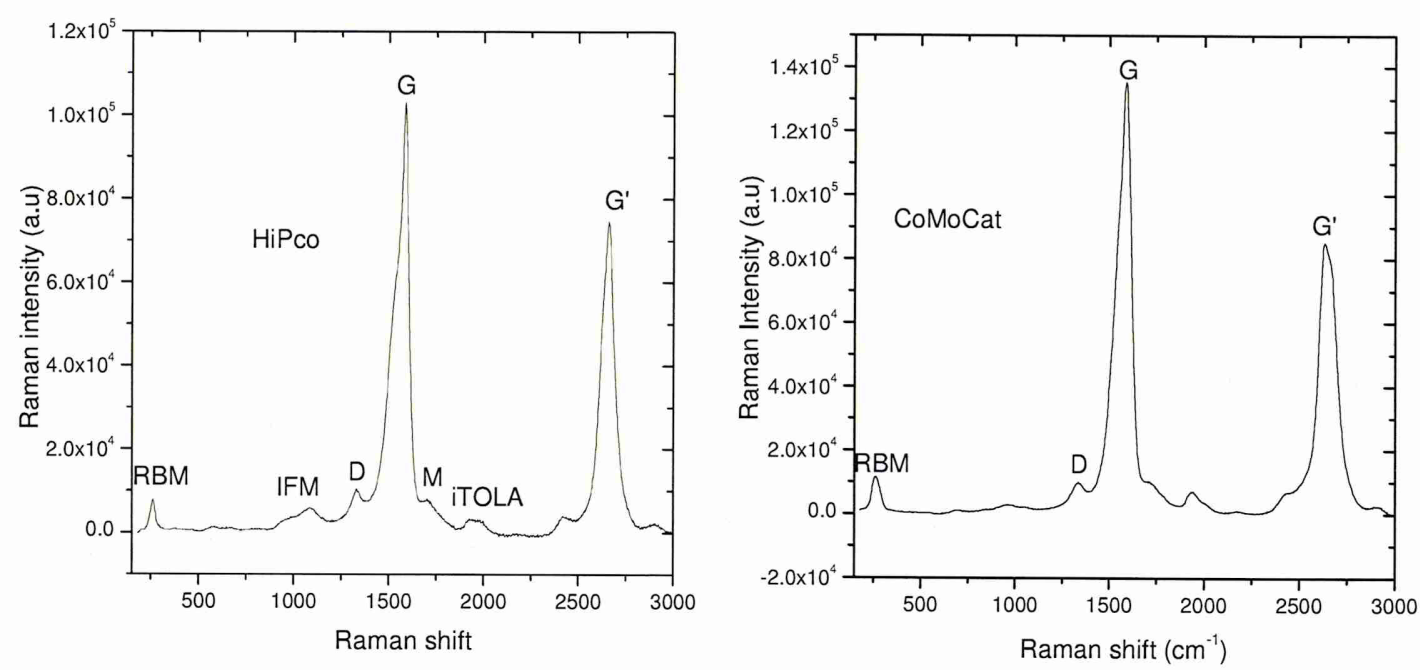

Figure 4-13: Raman spectra of SWNTs powder (a) HiPco powder (b) CoMoCat Powder. The Raman spectra show similar features for both SWNT samples.

\subsubsection{Raman Spectra of Dry SWNTs}

In Fig. 4-14, we plotted the Raman spectra of the dry SWNT (ss-DNA HiPco and CoMoCat1 sample) samples described in section 4.2. The dry SWNT samples were prepared by putting a drop of stock SWNTs solution onto a mica substrate and drying it at room temperature. Raman spectra of the dried samples is plotted from $200 \mathrm{~cm}^{-1}$ to $1800 \mathrm{~cm}^{-1}$. The background was subtracted from the raw Raman spectra before the fitting. The background subtracted Raman spectra fitted with multiple Lorentzians is shown in Fig. 4-14.

\section{G-band}

An important feature observed in the Raman spectra of dry SWNT samples is the tangential mode (G-band) which is a first order Raman process and a non-dispersive mode whose frequency does not depend on the laser excitation. The Raman spectra in the case of 
CoMoCat is characterised by a very sharp high frequency $G^{+}$band at $\sim 1590 \mathrm{~cm}^{-1}$ due to vibration of carbon atoms along the direction of nanotubes axis together with less intense $G^{-}$ band at $\sim 1560 \mathrm{~cm}^{-1}$ due to vibration along the circumferential axis [105], whereas in the case of ss-DNA wrapped HiPco SWNTs, apart from these two sub bands, there is another sub band around $\sim 1515 \mathrm{~cm}^{-1}$ named as $G_{m}^{-}$band. This band originates due to metallic nanotubes which are in resonance with the excitation wavelength $[28,107]$.
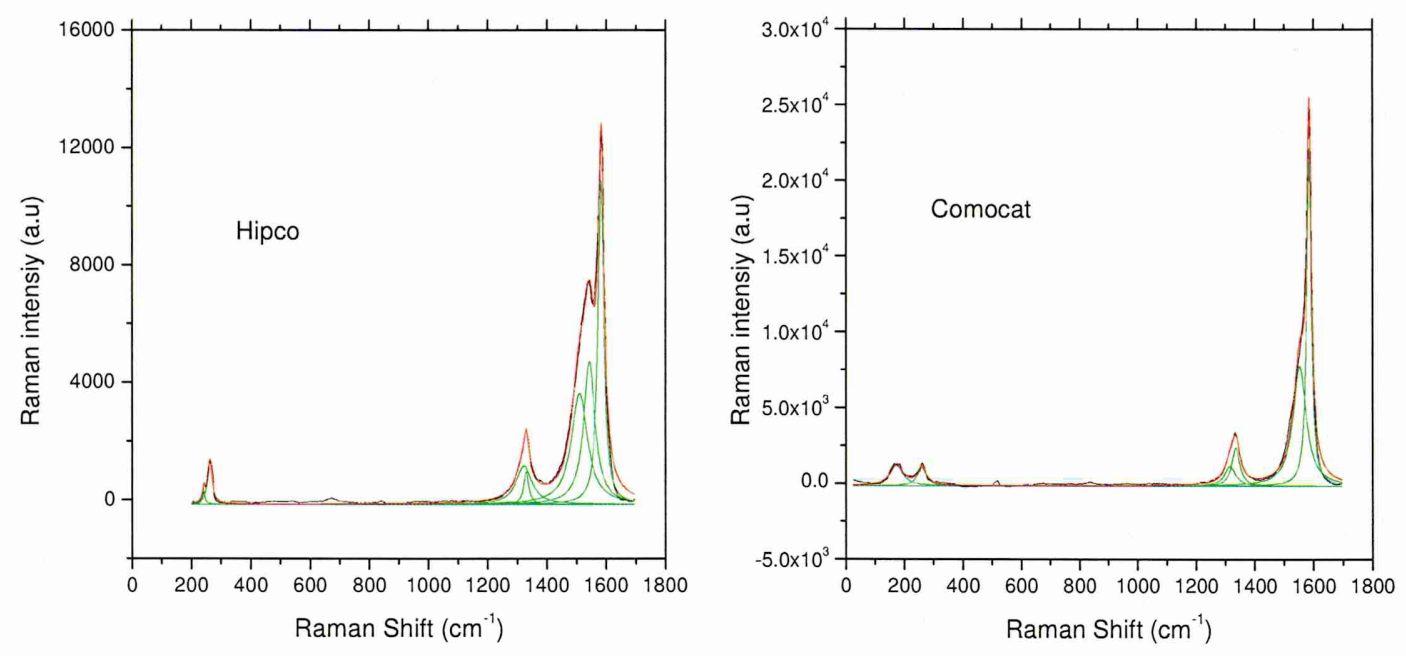

Figure 4-14: Raman Spectra of dry SWNTs. (a) ss-DNA wrapped HiPco SWNTs (b) CoMoCat1 SWNT sample. The features observed are discussed in the text.

\section{D-band}

Another important feature in Fig. 4-14 is the defective D band at $1330-1340 \mathrm{~cm}^{-1}$, a second order Raman scattering processes observed in both the semiconducting and metallic nanotubes. The $\mathrm{D}$ band has dispersive behaviour where the phonon frequency changes with laser excitation energy [108]. The D band in the spectra of $\mathrm{sp}^{2}$ carbon-based materials is the signature of defects in the graphene lattice and originates from a double resonance Raman scattering process $[109,110]$. In this process, an electron excited by the incident photon in an electron-hole pair creation process is inelastically scattered by emitting or absorbing a phonon. By means of a second, elastic, scattering with a defect, the electron may be scattered back to a point in k-space where its momentum is sufficiently near to that initial hole in order to radiatively recombine [111]. Hence the intensity of this band is dependent on the defect density. 


\section{Radial Breathing Mode (RBM)}

The presence of a radial breathing mode (RBM) in Fig. 4-14 is an indication that the Raman spectra originates from nanotubes, as RBM occurs only in carbon nanotubes in which all the carbon atoms vibrate coherently in the radial direction. The RBM is a first order Raman scattering process involved the emission of one phonon. The frequency of RBM $\left(\omega_{R B M}\right)$ lies between $120-350 \mathrm{~cm}^{-1}$ for the SWNTs with diameter $0.7 \mathrm{~nm}<d_{t}<2 \mathrm{~nm}$ and it scales with the diameter of the nanotubes according to the equation $[105,106,111]$

$$
\omega_{R B M}=A+\frac{B}{d_{n}}
$$

where the constant $A$ is included to account for the environment interaction i.e. tube-tube interaction in bundled nanotubes samples, and tube and substrate interaction. Its value is $\sim 15 \mathrm{~cm}^{-1}$ whereas in the case of isolated nanotubes in a suspension, its value is zero. The constant $B$ is found to be in the range of $217-227 \mathrm{~cm}^{-1}$ [112].
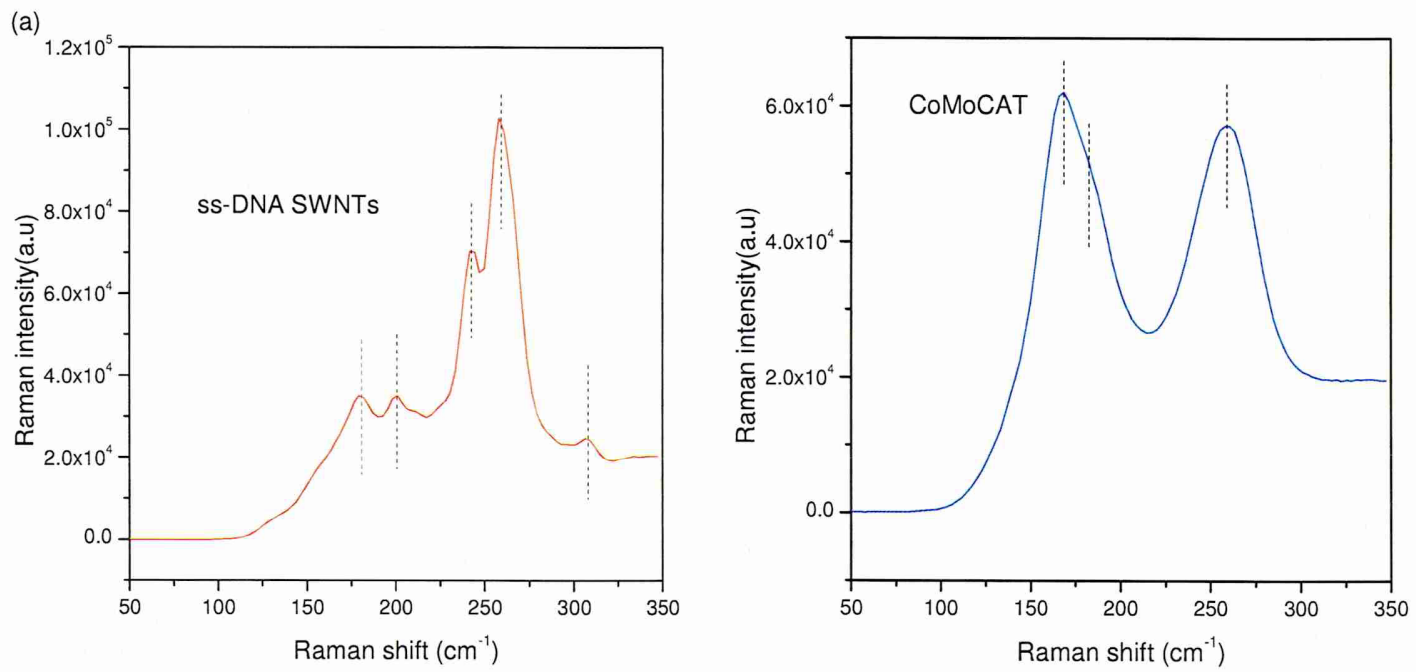

Figure 4-15: Radial breathing mode (RBM) bands of SWNTs (a) ss-DNA HiPco SWNTs (b) CoMoCat1 SWNTs.

In our case, we used RBM to identify the nanotube species which are excited during Raman spectroscopy. Intense multipeak RBM bands are observed for both DNA HiPco and CoMoCat1 SWNTs as shown in Fig. 4-15. The Fig. 4-15 shows that DNA HiPco SWNTs have five major peaks and CoMoCat1 SWNT sample has three major peaks. The assignment of these peaks to specific nanotube species $[28,107,113,114]$ is given in table $4-4$ which 
confirms the presence of metallic nanotubes in both SWNT samples.

\begin{tabular}{|c|c|c|c|c|c|c|c|}
\hline \multicolumn{5}{|c|}{ HiPco SWNTs } & \multicolumn{5}{c|}{ CoMoCat SWNTs } \\
\hline peak & Raman & SWNT & Diameter & peak & $\begin{array}{c}\text { Raman } \\
\text { shift }\left(\mathrm{cm}^{-1}\right)\end{array}$ & $\begin{array}{c}\text { SWNT } \\
(\mathrm{n}, \mathrm{m})\end{array}$ & $\begin{array}{c}\text { Diameter } \\
(\mathrm{nm})\end{array}$ \\
\hline 1 & 308.20 & $(6,5),(7,4)$ & $0.80,0.75$ & 1 & 258.40 & $(11,1)$ & 0.9 \\
\hline 2 & 259.00 & $(11,1)$ & 0.90 & 2 & 183.15 & $(11,8)$ & 1.29 \\
\hline 3 & 243.26 & $(11,3)$ & 1.01 & 3 & 167.41 & $(15,6)$, & 1.49, \\
& & & & & & $(16,4)$ & 1.46 \\
\hline 4 & 200.76 & $(15,0),(14,2)$, & $1.22,1.18$, & - & - & - & - \\
& & $(13,3)$ & 1.23 & & & & \\
\hline 5 & 180.78 & $(13,6),(15,3)$ & $1.36,1.31$ & - & - & - & - \\
\hline
\end{tabular}

Table 4-4: Assignment of RBM peaks to SWNT species which were excited during Raman spectroscopy.

The presence of metallic nanotubes in the RBM band of HiPco sample is consistent with our earlier explanation of an extra peak in the $\mathrm{G}$ band due to metallic nanotubes. Compared with RBM bands of starting ss-DNA HiPco SWNTs [28], sample still exhibit most of the peaks but with down shift of $1.2-3.0 \mathrm{~cm}^{-1}$ which may be due to lose wrapping of nanotubes.

The broadening of the peaks in the CoMoCat SWNT samples indicates that more than one nanotubes species are contributing to single peak. The exact number of contributing species was found through the fitting of Raman spectra with a set of Lorentzian functions. Fitting of the background-free spectra with Lorentzians is shown in Fig. 4-16.

Figure 4-16 shows that two extra peaks appeared in addition to five peaks discussed previously in the RBM bands of DNA HiPco SWNTs. These peaks are: peak 4 $\left(221.36 \mathrm{~cm}^{-1}\right)$ and peak $7\left(159.35 \mathrm{~cm}^{-1}\right)$ due to presence of nanotube species $(10,5)$ and $(19,1)$ with diameter $1.11 \mathrm{~nm}$ and $1.55 \mathrm{~nm}$ respectively. Also two extra peaks were observed in the case of CoMoCat nanotubes at $270.53 \mathrm{~cm}^{-1}$ and $244.60 \mathrm{~cm}^{-1}$ which are due to $(9,3)$ 
and $(7,7)$ tubes with diameter $0.93 \mathrm{~nm}$ and $1.01 \mathrm{~nm}$ respectively.
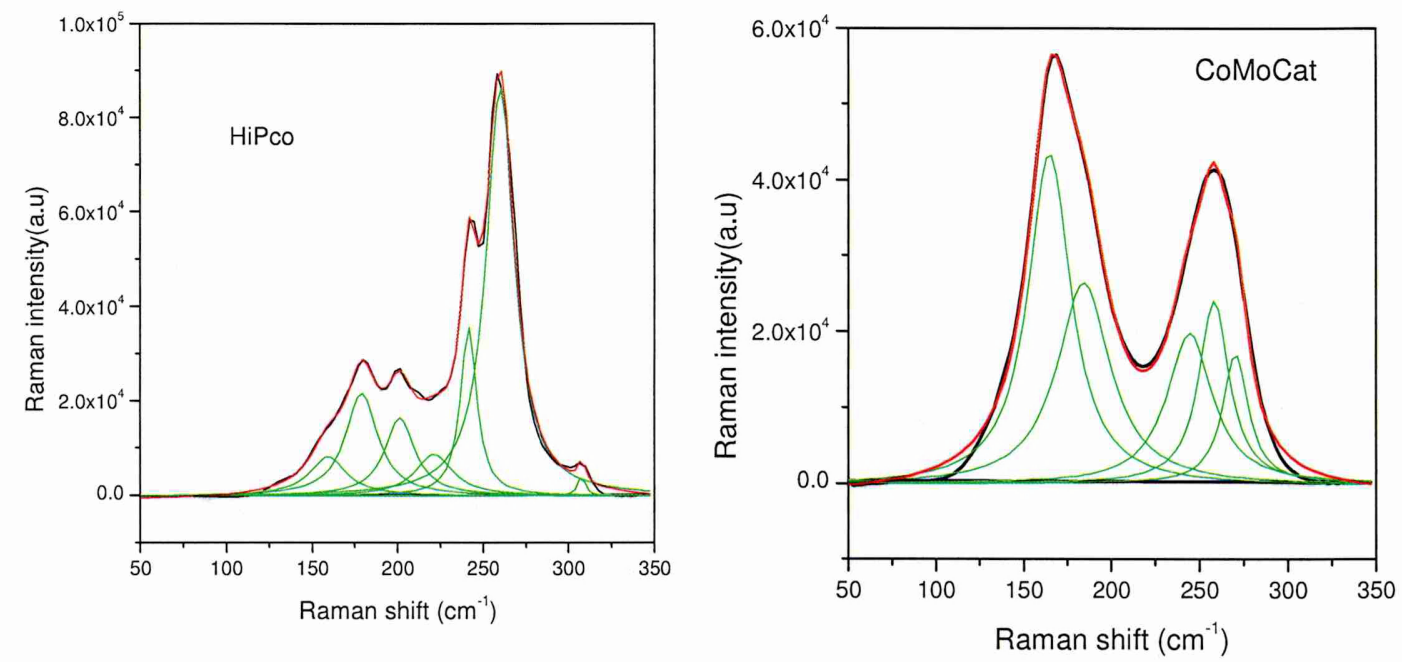

Figure 4-16: Radial breathing mode (RBM) bands of SWNTs fitted with of Lorentzians (a) ss-DNA wrapped HiPco SWNTs (b) CoMoCat1 SWNTs.

\subsubsection{Raman Spectra of SWNTs Solution}

To further examine these samples, Raman scattering was also carried out on solutions of SWNTs. The Spectrosil ${ }^{\mathrm{TM}}$ fused quartz cuvette $(1 \mathrm{~mm}$ path length) was selected to hold the SWNT suspension for these measurements. The Raman study was performed under the same condition for both samples in order to maintain the uniformity of intensity which is very important to compare the defect density in these samples. The background-free Raman spectra of SWNT suspension is shown in the Fig. 4-17.

The Raman spectra for both the samples are similar in terms of the number of peaks observed and also show similar features to those observed in the case of SWNT powder (e.g. see Fig. 4-13). The Raman spectra are plotted from $1200-3000 \mathrm{~cm}^{-1}$ which covers the important features (D band, G band, and $G^{\prime}$ band) of SWNTs which are used to study the defect concentration in these SWNT samples.

In Fig. 4-17, no evidence of broadening of $\mathrm{G}^{\prime}$ band (at $\sim 2700 \mathrm{~cm}^{-1}$ ) can be seen in the case of both ss-DNA HiPco and CoMoCat SWNTs which contradicts with Kalbac et al. [111] whom reported broadening of the $\mathrm{G}^{\prime}$ band in nanotubes which have more defects (due to higher intensity of $\mathrm{D}$ band). The possible reason is that the $\mathrm{G}^{\prime}$ band is a two phonon process which involves two inelastic scattering processes in contrast to the $\mathrm{D}$ band, where one of two 
scattering process involves scattering from a defect. Hence the D band is observed in all the $\mathrm{sp}^{2}$ carbon materials containing impurities, vacancies, and other symmetry-related defects. On other hand, $\mathrm{G}^{\prime}$ band is free from the elastic defect-related scattering process and is observed in defect free $\mathrm{sp}^{2}$ carbon materials despite being a second harmonic of the D band $\left(\omega_{G}^{\prime} \approx 2 \omega_{D}\right)[106,109,115]$.
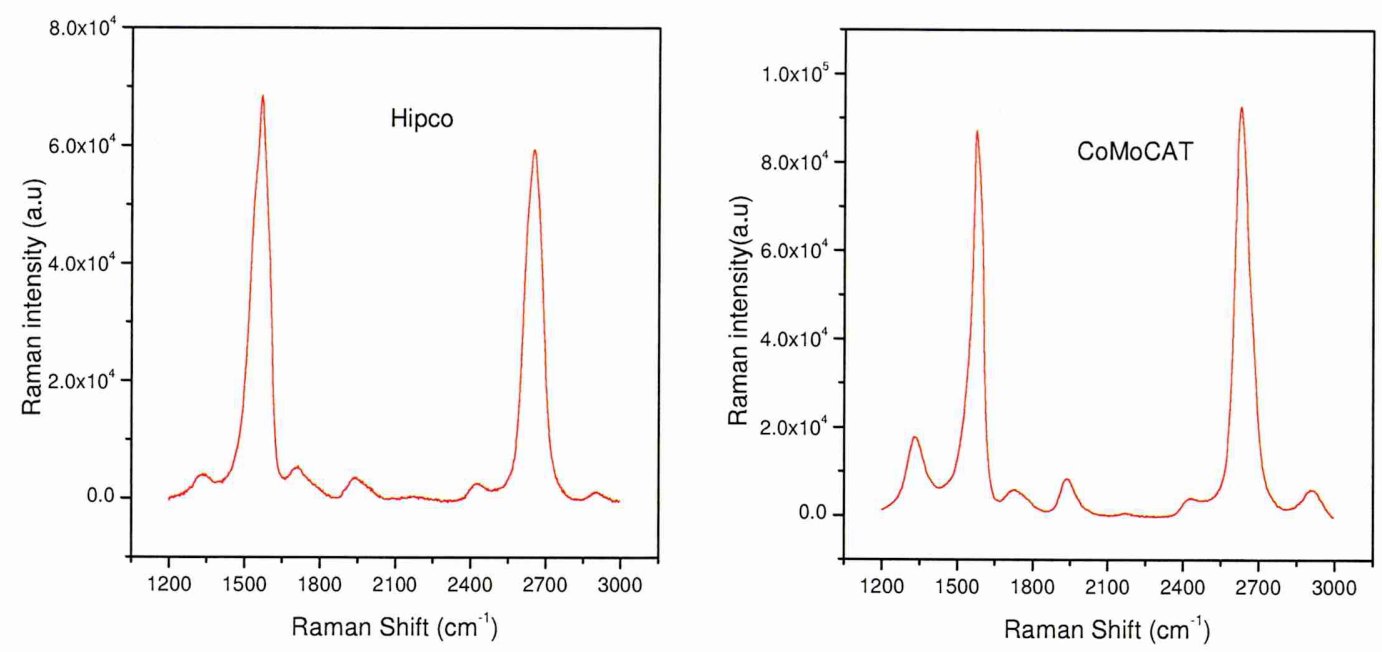

Figure 4-17: Background-subtracted Raman spectra of SWNT solution showing the same features as observed in the SWNT powder.

\section{Comparison of Raman Spectra of CoMoCat1 SWNTs and ss-DNA HiPco SWNTs}

\section{Defective D and Tangential G mode}

In this section, we present a comparison of the defective $\mathrm{D}$ band and tangential $\mathrm{G}$ band for the two different samples of SWNTs discussed above. This comparison is very important to quantify the defect concentration in these samples in order to understand the pump probe results discussed in chapter 6 . The Raman spectra of the SWNTs in solution are plotted again in Fig. 4-18 in order to compare the defect density in each sample. This can be done by comparing the ratio of $\mathrm{D}$ band and $\mathrm{G}$ band.

As we discussed above, the $\mathrm{G}$ band has sub bands $\mathrm{G}^{+}, \mathrm{G}^{-}$due to semiconducting nanotubes and $G_{m}^{-}$due to metallic nanotubes. The $\mathrm{G}^{+}$band originates from the longitudinal optical phonons of semiconducting nanotubes and is insensitive to laser excitation, diameter of nanotubes, and the presence of defects [108], while the lower frequency $\mathrm{G}^{-}$band is dependent on the diameter. A combination of Gaussians and Lorentzians was used to fit the Raman data 
shown in Fig. 4-18.
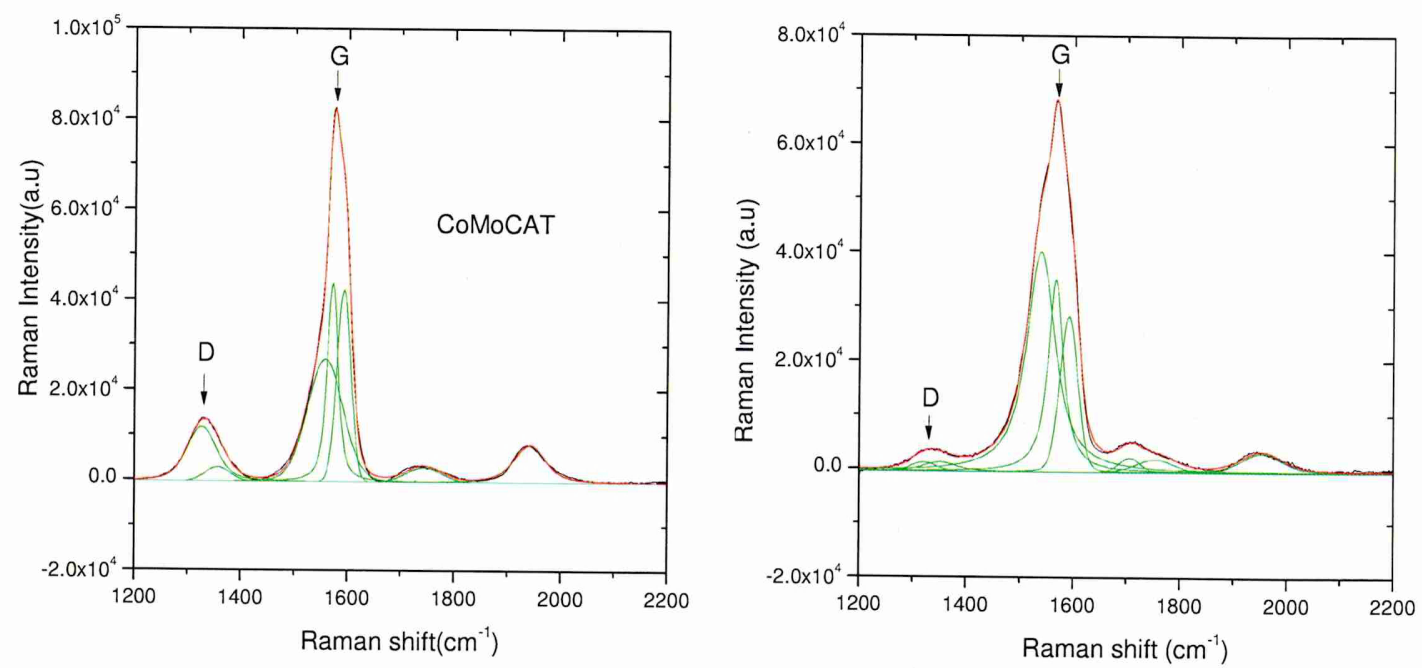

Figure 4-18: Raman study of CoMoCat1 sample and HiPco SWNTs. $I_{D} / I_{G}$ was compared to study the defect in these samples. The black line is the experimental data and red line is fit to the data using the combination of Gaussian and Lorentzian functions. The green lines represent the position of each fitted peak.

The $\mathrm{G}^{-}$band frequency can be used to find the diameter of the nanotubes in which the radial breathing mode does not occur through following relation [106]

$$
\omega_{G}^{-}=\omega_{G}^{+}-\frac{\beta}{d_{n}^{2}}
$$

where $\omega_{G}^{-}, \omega_{G}^{+}$, and $\beta$ are the frequency of $\mathrm{G}^{-}$band, $\mathrm{G}^{+}$band, and coefficients of the diameter respectively. The value of $\beta$ is $45.7 \mathrm{~cm}^{-1} \mathrm{~nm}^{2}$ for semiconducting nanotubes and $79.5 \mathrm{~cm}^{-1} \mathrm{~nm}^{2}$ for metallic nanotubes. The diameter of semiconducting and metallic nanotubes determined from $\mathrm{G}^{-}$band frequency using the equation 4.4 is given in the table $4-5$

\begin{tabular}{|c|c|c|c|c|c|}
\hline $\begin{array}{c}\text { Dry } \\
\text { Samples }\end{array}$ & Nanotubes & $\begin{array}{l}\text { Diameter } \\
(\mathrm{nm})\end{array}$ & $\begin{array}{l}\text { Solution } \\
\text { Samples }\end{array}$ & Nanotubes & $\begin{array}{c}\text { Diameter } \\
(\mathrm{nm})\end{array}$ \\
\hline \multirow[b]{2}{*}{ HiPco } & Semiconducting & 1.08 & \multirow[b]{2}{*}{ HiPco } & Semiconducting & 1.36 \\
\hline & Metallic & 1.05 & & Metallic & 1.23 \\
\hline \multirow[b]{2}{*}{ CoMoCat } & Semiconducting & 1.19 & \multirow[b]{2}{*}{ CoMoCat } & Semiconducting & 1.47 \\
\hline & Metallic & - & & Metallic & 1.51 \\
\hline
\end{tabular}

Table 4-5: Diameter of nanotubes determined from $G^{-}$band frequency using equation 4.4. 
The ratio of intensity of $\mathrm{D}$ band and $\mathrm{G}^{+}$band is used to evaluate the defect density in our samples [111]. The $I_{D} / I_{G}$ ratio of CoMoCat $S W N T$ is found to be higher by factor of two than ss-DNA wrapped HiPco nanotubes (see table 4-6) which indicates that these CoMoCat nanotubes have more defects as compared to the HiPco nanotubes.

\begin{tabular}{|l|c|c|c|c|}
\hline \multirow{2}{*}{ Sample } & \multicolumn{2}{|l|}{ HiPco SWNTs } & \multicolumn{2}{l|}{ CoMoCat SWNTs } \\
\cline { 2 - 5 } & $\mathrm{A}_{\mathrm{D}} / \mathrm{A}_{\mathrm{G}}$ & $\mathrm{I}_{\mathrm{D}} / \mathrm{I}_{\mathrm{G}}$ & $\mathrm{A}_{\mathrm{D}} / \mathrm{A}_{\mathrm{G}}$ & $\mathrm{I}_{\mathrm{D}} / \mathrm{I}_{\mathrm{G}}$ \\
\hline Sample 1 & 0.26 & 0.09 & 0.48 & 0.21 \\
\hline Sample 2 & 0.16 & 0.08 & 0.33 & 0.15 \\
\hline
\end{tabular}

Table 4-6: Comparison of ratio of D band to $G$ band for ss-DNA HiPco and CoMoCat SWNTs. Here $A$ is area and I is intensity of fitted peak.

The average distance between the defects in SWNTs can be estimated using similar approach to Kalbac et al. [111] who reported $\sim 25 \mathrm{~nm}$ defect separation for similar value of $I_{D} / I_{G}$. However, main source of quantifying the defects was reported for graphene by Lucchese et al. [116] using areal density of defect which might be true for two dimensional graphene but is not probably appropriate for 1D SWNTs i.e.

$$
\frac{I_{D}}{I_{G}}=\frac{(102 \pm 2) n m^{2}}{s^{2}}
$$

where $S$ is separation between defects. Using the equation 4.5 , the average distance between defects for CoMoCat SWNTs is estimated to be $s \sim 22 \mathrm{~nm}$ and for HiPco SWNT, it is approximately $s \sim 40 \mathrm{~nm}$.

\subsection{X-ray Photoelectron Spectroscopy (XPS) of SWNTs}

X-ray photoelectron spectroscopy of SWNTs was carried out to measure the defect concentration in SWNTs. XPS study has been performed with an $\mathrm{Al} \mathrm{K}$ alpha X-ray source with photon energy of $1486.6 \mathrm{eV}$. Survey scans were acquired at a pass energy of $300 \mathrm{eV}$. The 
survey spectrum of DNA HiPco SWNTs is shown in Fig. 4-19.

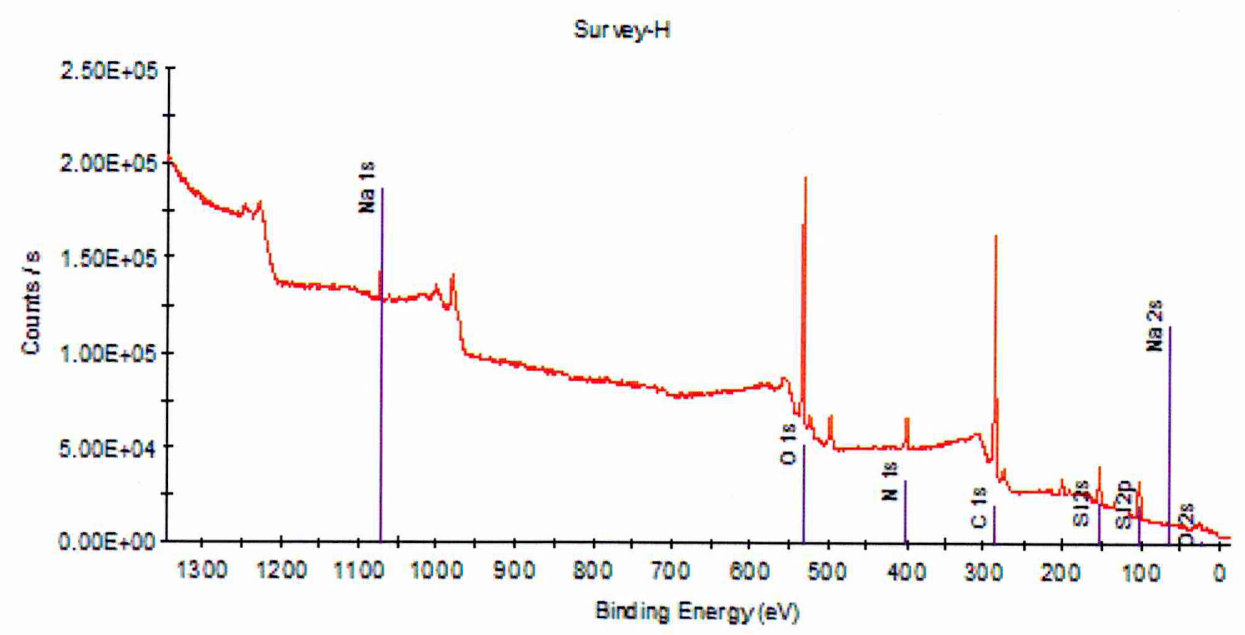

Figure 4-19: Survey XPS spectrum of HiPco SWNTs.

The XPS spectrum in Fig. 4-19 shows that there is no definite peak around the $707 \mathrm{eV}$ and $720 \mathrm{eV}$ due to residual metallic $F e 2 p_{1 / 2}$ and $F e 2 p_{3 / 2}$ respectively which was reported before for HiPco SWNTs $[117,118]$ and also no sign of $F e$ 2s peak at $845 \mathrm{eV}$ [119]. The absence of catalytic iron particle in the XPS spectrum demonstrates that our DNA wrapped HiPco SWNTs are purified nanotubes.

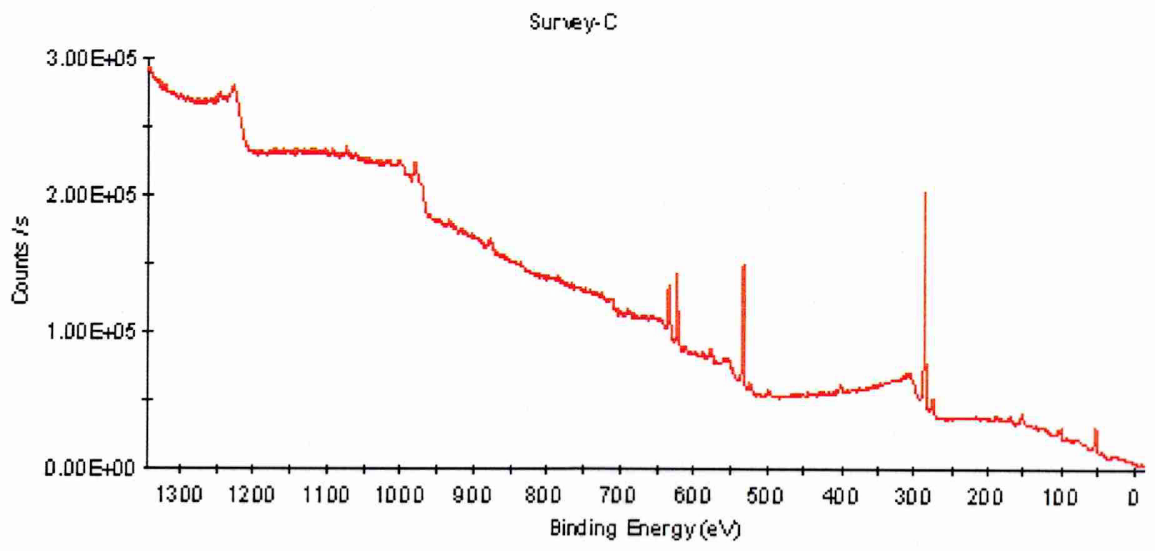

Figure 4-20: XPS survey of CoMoCat SWNTs, showing the different peaks.

In Fig. 4-20, the XPS spectrum of CoMoCat SWNTs in 2\% sodium Cholate (SC) is plotted. Again there is no sign of the peaks around $925 \mathrm{eV}, 793.2 \mathrm{eV}$, and $778.1 \mathrm{eV}$ due to 
cobalt $\operatorname{Co} 2 s, \operatorname{Co} 2 p_{1 / 2}$, and $\operatorname{Co} 2 p_{3 / 2}$ respectively. Furthermore, Fig. 4-20 shows that CoMoCat has no XPS peaks at $411.6 \mathrm{eV}$ and $394.0 \mathrm{eV}$ due to molybdenum $M o 3 p_{1 / 2}$ and Mo $3 p_{3 / 2}$ respectively [119]. This indicates that our CoMoCat SWNTs are also pure and no catalytic residual particles were left during the purification process.

\subsubsection{Comparison of Defects in SWNTs}

The presence of defect concentration in each sample can be found by comparing the $\mathrm{C} 1 \mathrm{~s}$ peak of each sample. Narrow scan C 1s spectra were recorded at a pass energy of $50 \mathrm{eV}$ and are plotted in Fig. 4-21 for both ss-DNA wrapped HiPco and CoMoCat1 SWNTs. Both samples show a peak associated with graphitic carbon $(\sim 284.6 \mathrm{eV})$ and additional peaks in the range $286-290 \mathrm{eV}$.
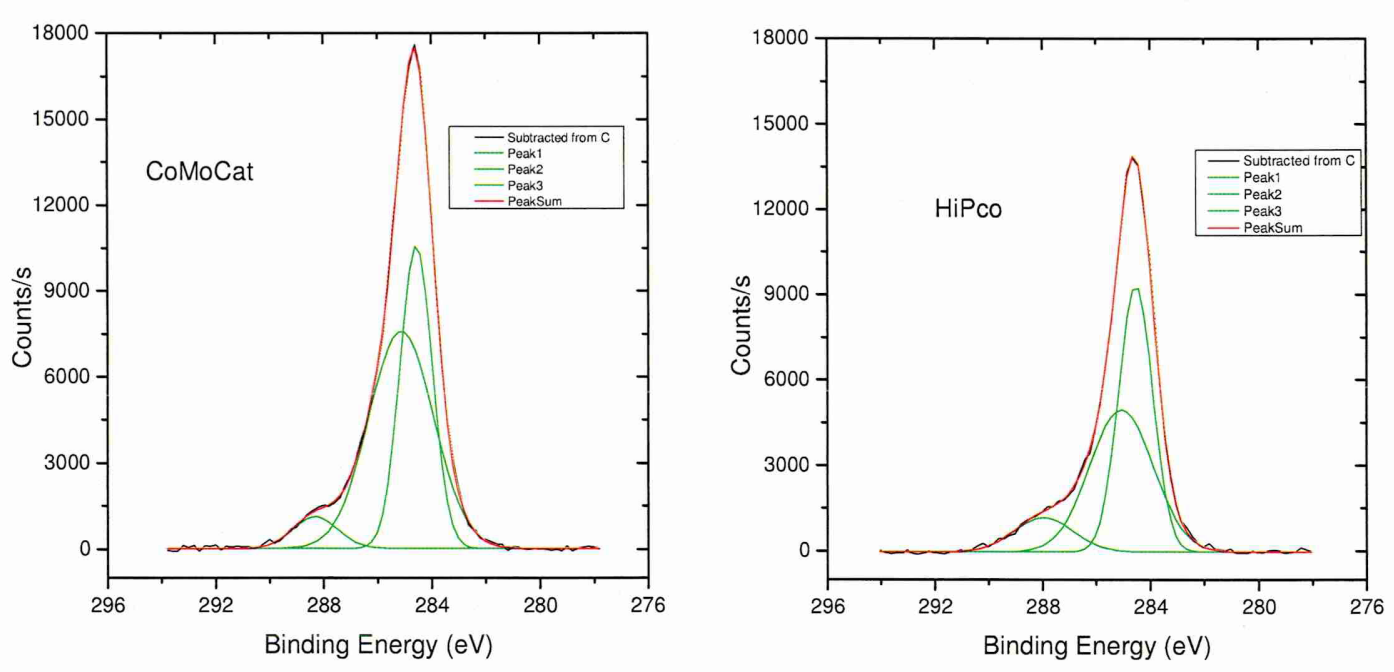

Figure 4-21: XPS C1s spectra of SWNTs fitted with three Gaussians (a) CoMoCat1 SWNTs in sodium cholate (b) ss-DNA wrapped HiPco SWNTs.

Fitting of XPS spectra of ss-DNA wrapped HiPco and CoMoCat1 SWNTS with Gaussian functions (Fig. 4-21) shows that the $\mathrm{C} 1 \mathrm{~s}$ peak can be resolved into three peaks, peak 1 at $284.5 \mathrm{eV}$ represents bonding of $\mathrm{C}$ atoms to a graphitic network ( $\mathrm{sp}^{2}$ bonding), peak 2 at $\sim 285.1 \mathrm{eV}$ appeared due to bonding of $\mathrm{C}$ atoms at defects ( $\mathrm{sp}^{3}$ bonding) as the dangling bonds appear at higher binding energy compared to that of graphitic C atoms [120, 121], and peak 3 at $\sim 288.2 \mathrm{eV}$ is due to carboxyl $(\mathrm{COOH})$ or ester groups [122]. The relative size of the defectrelated peak 2 is seen to be larger in the CoMoCat sample. 
FWHM and area of the defect-related $\mathrm{sp}^{3}$ peak 2 and the graphitic $\mathrm{sp}^{2}$ peak 1 are listed in table 4-7. Both the FWHM and the area are higher in the case of CoMoCat SWNTs as compared to ss-DNA HiPco SWNTs. Yoon et al. [120] established a relationship between the FWHM and area with the defect density in carbon nanotubes. Hence our XPS results confirmed that these CoMoCat nanotubes have more defects than the DNA-wrapped HiPco SWNTs.

\begin{tabular}{|l|c|c|c|c|c|}
\hline \multirow{2}{*}{ Sample } & \multicolumn{2}{|c|}{ Peak 1 (graphite) } & \multicolumn{2}{c|}{$\begin{array}{c}\text { Peak 2 } \\
\text { (defect) }\end{array}$} & \multirow{2}{*}{$A_{s p^{3}} / A_{s p^{2}}$} \\
\cline { 2 - 5 } & FWHM (eV) & Area & FWHM (eV) & Area & \\
\hline HiPco & 1.4 & 14740 & 2.76 & 14677 & 1.00 \\
\hline CoMoCat & 1.4 & 15754 & 2.82 & 22694 & 1.44 \\
\hline
\end{tabular}

Table 4-7: FWHM and area obtained from C1s peaks of HiPco and CoMoCat1 SWNTs, showing that area as well FWHM of $\mathrm{sp}^{3}$ peak of CoMoCat is higher than HiPco which indicates that CoMoCat nanotubes have more defects.

\subsection{Summary}

In this chapter, we have shown that the isolation of SWNTs can be achieved through DNA wrapping as well through use of a surfactant e.g. sodium cholate. We have shown through linear optical absorption measurement and AFM measurements that nanotubes are well isolated from each other. The isolation of SWNTs allows us to identify the constituent species in the sample through comparison of peak position observed in the linear absorption spectrum with that of tabulated energies given in [99]. AFM study of HiPco SWNTs reveals that nanotubes are isolated through helical wrapping of DNA.

Comprehensive Raman spectroscopy was performed to compare the defects in SWNTs. Our Raman results clearly show that defective nanotubes contribute more to the intensity of defective band ( $\mathrm{D}$ band) as compared to less defective nanotubes. In short, Raman spectroscopy confirmed that these CoMoCat nanotubes have more defects than HiPco SWNTs. We have also shown from an XPS study that our SWNT samples are pure and no residual catalytic impurities were left after purification. The defects in CoMoCat nanotubes were found to be higher than HiPco SWNTs through the comparison of FWHM and area of $\mathrm{sp}^{3}$ and $\mathrm{sp}^{2}$ peaks using the XPS $\mathrm{C} 1 \mathrm{~s}$ spectra. 


\section{CHAPTER 5}

\section{PUMP PROBE MEASUREMENTS OF HIPCO SWNTS}

In this chapter, we investigate the decay dynamics of photogenerated excitons in nanotubes with relatively low concentration of defects. This chapter is organised in such a way that in section 5.1, we present the experimental results, including the influence of the pump pulse intensity. In section 5.2, a comprehensive quantitative analysis of the results is given where we present a methodology for determining the exciton population from transmission change. In the same section, we also show the universality of the universal behaviour indicated by independence on initial concentration at long time. The interpretation of results is given in section 5.3, where we describe exciton reactions on SWNTs as an archetypal reaction-diffusion system. We review the evidence for distinct regions of reactionlimited and diffusion-limited behaviours and extract reaction rate constant and diffusion coefficient from fitting of experimental data with asymptotic rate equations. The dynamical crossover between reaction-limited and diffusion-limited regimes and long range interaction of excitons on carbon nanotubes is also discussed in this section. A summary of results is given in last section (section 5.4). 


\subsection{Experimental Results and their Qualitative Interpretation}

In this section of the chapter, we investigate the decay of photogenerated excitons in a solution of separated ss-DNA wrapped HiPco SWNTs. This information is gathered through standard degenerate pump probe measurements performed at $1132 \mathrm{~nm}$ which is in resonance with $E_{11}$ transition of $(7,6),(9,2)$, and $(8,4)$ species (diameter $\sim 0.9 \mathrm{~nm}$ ) as identified in chapter 4 from optical absorption characteristics. We found this sample to be very stable against agglomeration for a long period of time more than several months and to contain uniformly distributed well isolated SWNTs as shown by optical absorption and AFM measurements as discussed in chapter 4 . This stability of the sample permitted us to repeat the full set of pump probe measurements with the same results but increased precision and reduced noise, in order to increase our confidence in the experimental results and in our interpretation. These later results are the ones presented here.

A quantitative analysis of the experimental results requires that the measured transmission change should be converted into an exciton population. This can be done approximately if the optical fluence, density of nanotubes, and absorption coefficient per nanotube can be estimated (although the absorption is in fact highly nonlinear). In order to avoid these approximations, in this section we consider ways to represent the data in such a way that qualitative conclusions can be obtained from the transmission changes alone. In the following section, we will present a methodology for determining the exciton population from transmission change without any such approximations; this will allow a quantitative interpretation of the data and yields precise values for reaction rate constants, diffusion coefficients, and other parameters.

In Fig. 5-1, the differential transmission $(\Delta T)$ is plotted as function of time for the highest pump power of $26 \mathrm{~mW}$ at a repetition rate of $250 \mathrm{kHz}$. The black dots in this figure are values of individual scans and the red curve is mean value of all individual scans. In part (a), the transmission is plotted as a function of time, on linear scales. The signal is characterised by ground state photobleaching at $t=0$, where reduced absorption is observed (increased transmission) due to transfer of exciton population from ground state to first excited state. A negative dip is seen at the negative time just before zero time delay which may be due to absorption of hot carriers.

In part (b), logarithmic transmission is plotted; the blue curve in this part is the "standard error in the mean". The exact position of temporal overlap (zero time delay i.e. $t=0$ ) of 
pump and probe beams was measured from the position of the peak in the standard error which appeared due to interference fringes of pulses. As described in chapter 3, special care was taken to both minimise and accurately measure the background signal due to pump scattering. For this case of maximum pump intensity where the pump scattering is highest, the background signal measured at negative time (arrow in part (b)) is $<1 \%$ of the maximum signal. After 10 repeated scans the standard error in the background is $\sim 6.5 \times 10^{-4}$ of the maximum signal. The background signal can therefore be subtracted to a high degree of precision.
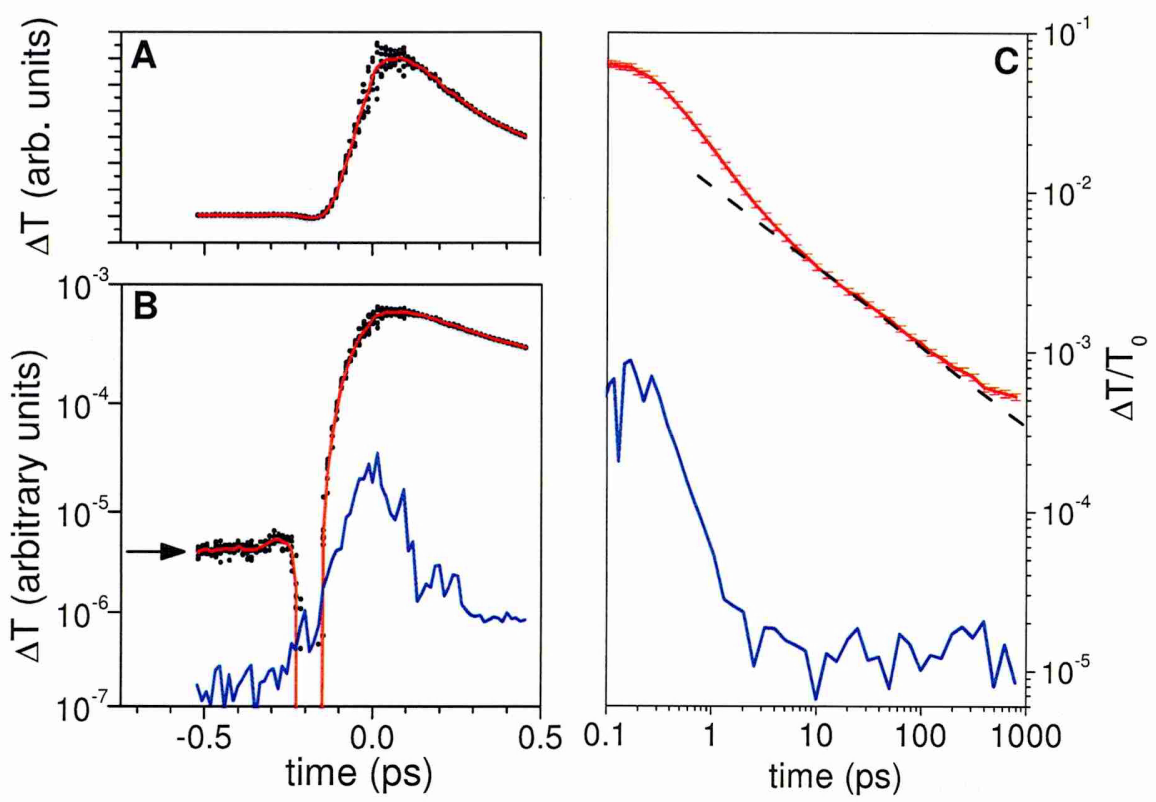

Figure 5-1: Differential transmission at high pump power of $26 \mathrm{~mW}$. The black dots are values of individual scans and red curves are the mean value of individual scans. The blue line is standard error in the mean whose peak value determined the time zero delay of pump and probe beam (a) linear transmission, (b) logarithmic of transmission, (c) log-log plot of normalised differential transmission. Error bars are combination of the standard error in the measured transmission and in the background. Dashed line indicates $t^{-1 / 2}$ power law and is included here as guide to the eye.

In part (c) of this figure, log-log plot of normalised differential transmission $\left(\frac{\Delta T}{T_{0}}\right)$ (which we called signal " $\mathrm{S}$ " in this thesis) is plotted for a wide range of time up to $\sim 1 \mathrm{~ns}$. The differential transmission $(\Delta T)$ is obtained from the probe pulse transmission when the pump 
pulse precedes the probe pulse (probe transmission at positive time i.e. $t \geq 0$ ), less the probe pulse transmission when the probe pulse precedes the pump pulse (at negative time i.e. $t<0$ ) i.e. $\Delta T=\left(\Delta T_{\text {pos.time }}-\Delta T_{\text {neg.time }}\right)$ and $T_{0}$ is obtained from the probe pulse transmission when the pump pulse is off. In this figure, the red curve is the mean value of all individual scans and blue curve is standard error in the mean. The error bars are a combination of the standard errors in the measured transmission and in the subtracted background signal. The dotted line indicates the $t^{-1 / 2}$ power law decay shown as a guide.

This part reveals three different regimes clearly. (1) At time between 100 fs and 3 ps, a power law decay with faster decay than $t^{-1 / 2}$ is observed. (2) At time between $10 \mathrm{ps}$ and $100 \mathrm{ps}$, a clear $t^{-1 / 2}$ power law decay is observed which extends over approximately two orders of magnitude in time. (3) As the time approaches $1 \mathrm{~ns}$, a deviation from $t^{-1 / 2}$ power law decay is observed and the decay becomes much slower which we attribute to the low density situation when there is only one exciton remaining on each SWNT.

In Fig. 5-2, fluence dependent differential transmission $\left(\Delta T / T_{0}\right)$ is plotted as function of time to see the effect of saturation at early times. In Fig. 5-2 (a), differential transmission is plotted on linear scales and in Fig. 5-2 (b), logarithmic of differential transmission is plotted. This figure clearly shows the saturation of signal at high pump fluences. In addition to this saturation, the peak of the signal shifts in time at different pump fluences.
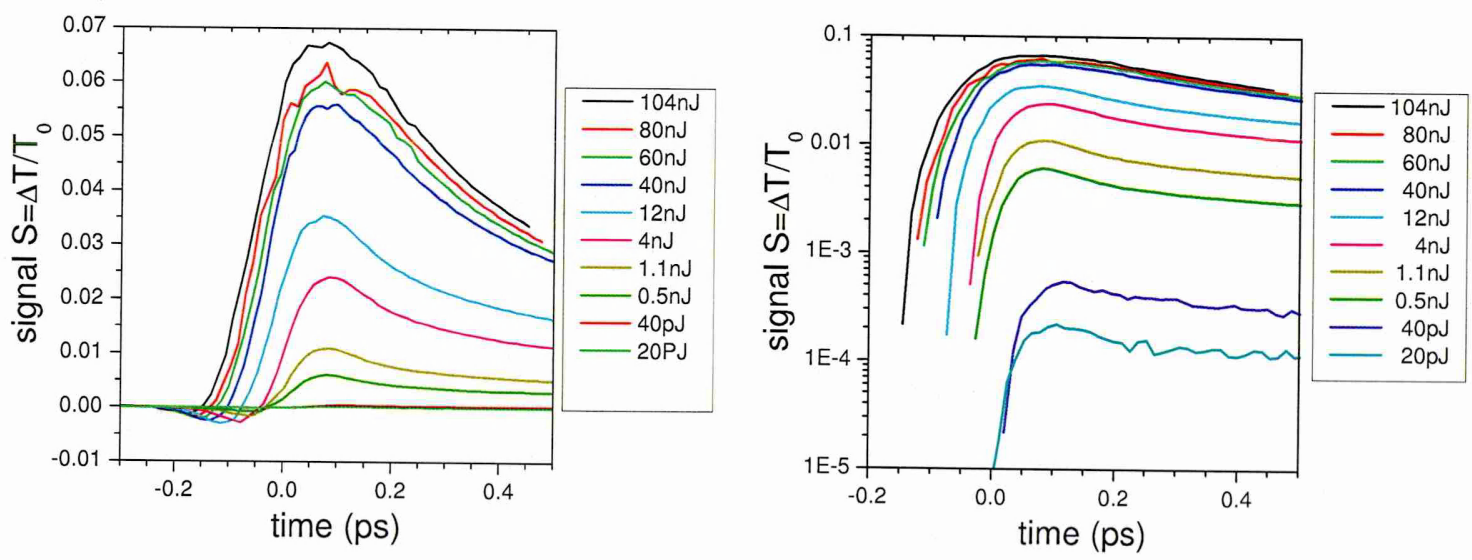

Figure 5-2: Pump fluence dependent differential transmission as function of pump probe delay, showing saturation of signal at high pump fluences, and shift in peak position of signal from zero time delay with pump fluences, (a) differential transmission on linear scales $(b)$ logarithmic of differential transmission. 
At low pump intensity, this shift is $\sim 150$ fs from time zero which possibly indicates the duration (length) of exciting pulse as peak position is determined by the integration of exciting pulse, whereas at high intensity, the peak occurs approximately at time zero and is presumably related to saturation of signal which occurs at approximately half way through the pulse. We ruled out effect of decay rate from our discussion and will show later that reaction does not occur during duration of the pulse even at high pump fluences.

In Fig. 5-3 we plot differential transmission $\left(\Delta \mathrm{T} / \mathrm{T}_{0}\right)$ taken at $1132 \mathrm{~nm}$ for different pump fluences that varies over three orders of magnitude from $3.97 \times 10^{-6} \mathrm{~J} / \mathrm{cm}^{2}$ to $3.68 \times$ $10^{-3} \mathrm{~J} / \mathrm{cm}^{2}$. The Fig. 5-3 shows that at times greater than $\sim 4 \mathrm{ps}$; the decay of the photoexcited population follows approximately a $t^{-1 / 2}$ power law for all the optical densities used. At times approaching $1 \mathrm{~ns}$ a positive deviation from $t^{-1 / 2}$ power law is seen, whose magnitude varies with fluence. At early time and high intensity, a decay with positive deviation from the $t^{-1 / 2}$ power law is seen, while at lower fluence this initial decay becomes slower than $t^{-1 / 2}$.

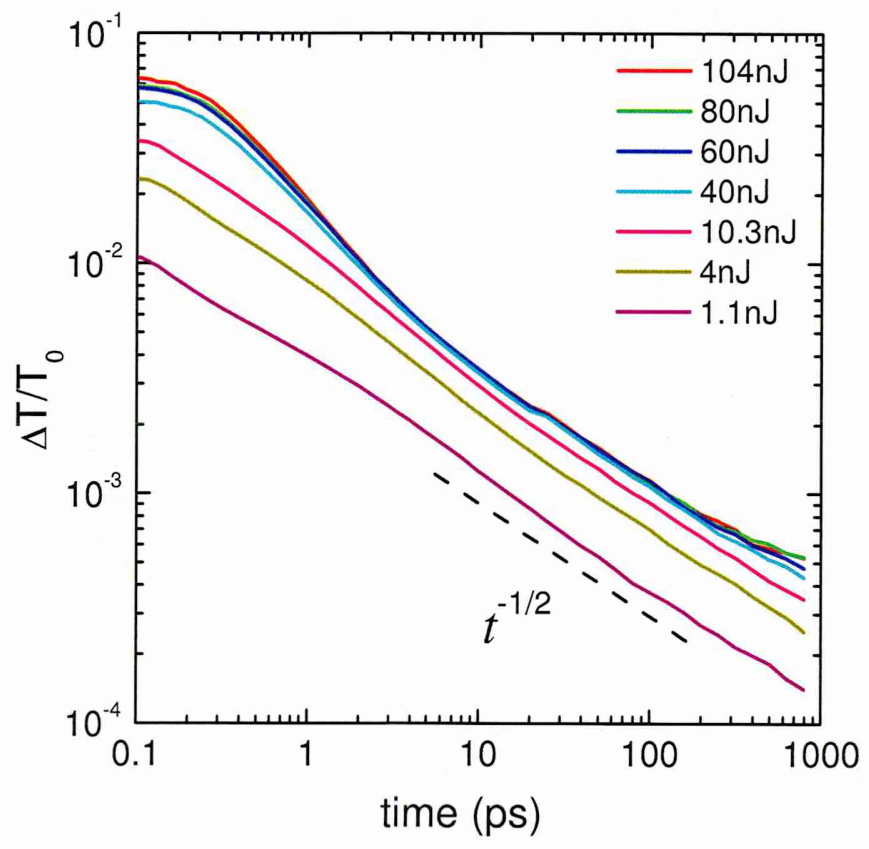

Figure 5-3: Fluence dependent pump probe data as function of pump probe delay up to $1 \mathrm{~ns}$, dashed line indicates the $t^{-1 / 2}$ decay at long time. All the traces follow the $t^{-1 / 2}$ power law at long time regardless of optical excitation densities. Deviation from this $t^{-1 / 2}$ power law is seen around $\sim 1 \mathrm{~ns}$, where the decay becomes slow. 
The differential transmission data plotted in Fig. 5-3 suggests that the exciton decay follows a power law $n(t) \propto t^{-|\alpha|}$ at early time $(100 \mathrm{fs} \leq t \leq 3 \mathrm{ps})$ and at long time $3 \mathrm{ps} \leq t \leq 100 \mathrm{ps}$, hence we determine the dimensionless decay exponent $|\alpha|=-(t / n)(d n / d t)$ by numerical differentiation of the experimental data and plot it as function of delay time in Fig. 5-4. The main objective of plotting experimental data in this way is to get information about decay exponents irrespective of scaling factors which may vary with pump fluence.

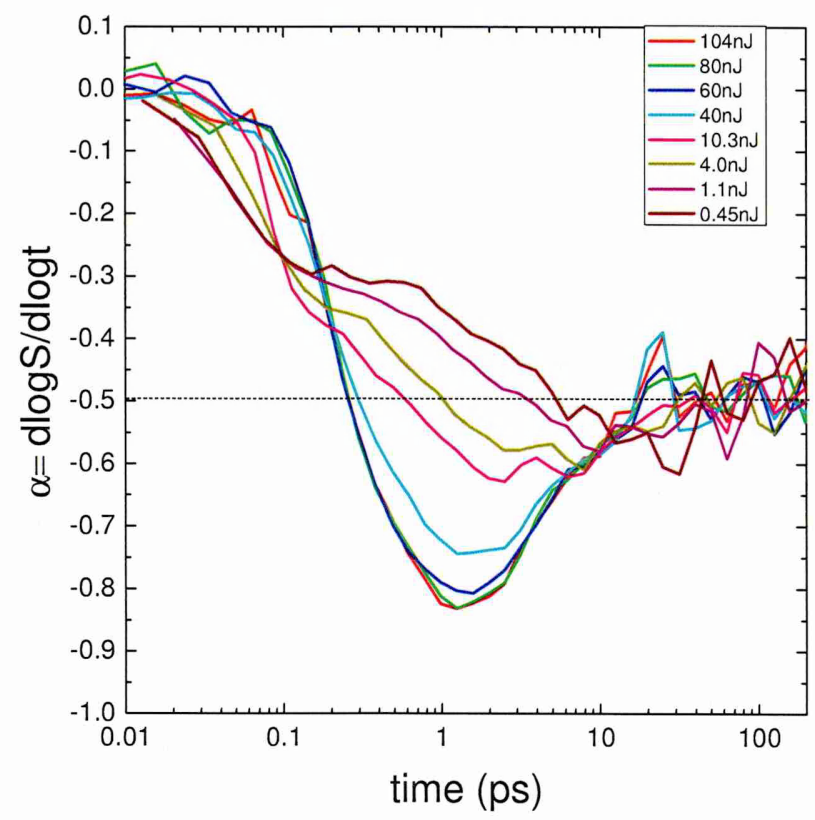

Figure 5-4: Logarithmic derivative of $S\left(\Delta T / T_{0}\right)$, equal to exponent ' $\alpha$ ' of a power law $t^{-|\alpha|}$. The dashed line indicated the $t^{-1 / 2}$ power law decay with decay exponent $\alpha=0.5$.

Figure 5-4 shows that the decay exponent saturates to -0.5 after 20 ps regardless of the initial excitation. This indicates the asymptotic region of anomalous kinetics with the expected universal decay exponent. At intermediate times (several ps), there is an increase in the magnitude of the exponent, reaching a maximum of $\alpha \sim-0.83$. At early times $(<0.1 \mathrm{ps})$, the exponent approaches zero because there is no decay on timescales short compared to the reaction $\left(t_{1}\right)$ and diffusion $\left(t_{0}\right)$ times (detail is given at end of this chapter). The expected value of $\alpha=-1$ for the reaction-limited regime is not observed, and it is not clear from Fig. 5-4 alone that whether this is due to saturation of initial exciton population at high excitation density, or the transition to the early $\alpha=0$ region (i.e. the initial condition), or the effect of 
some other additional process at early times. Also as the excitation decreases from the maximum value, the behaviour does not follow that predicted in [54], where the $\alpha=0$ region extends to longer time as $t_{0}+t_{1}$ increases with decreasing intensity. We discuss the later in terms of non-uniform excitation of the ensemble of nanotubes.

To further substantiate the nature of responses observed at early and long time, we plotted the time derivative of the experimental signal $\mathrm{S}=\Delta \mathrm{T} / \mathrm{T}_{0}$ as a function of $\mathrm{S}$ in Fig. 5-5. This has two benefits. Firstly, it removes the integration constant (i.e. the dependence on initial conditions), which is one of the possible reasons for not observing a $t^{-1}$ power law decay in Figures 5-3 and 5-4. Secondly, it allows a direct study of the dependence of rate equations on exciton density. From Fig. 5-5, we find that (1) at all excitation densities, there is an intermediate region where $\mathrm{dS} / \mathrm{dt}$ varies with $\mathrm{S}^{3}$, (2) at low signals, corresponding to small exciton populations, there is a decrease in $\mathrm{dS} / \mathrm{dt}$ compared to the above $\mathrm{S}^{3}$ dependence, (3) at the maximum excitation density, the decay rate $\mathrm{dS} / \mathrm{dt}$ approaches $\mathrm{S}^{2}$ at large $\mathrm{S}$ corresponding to maximum exciton populations, (4) at low excitation density and maximum $\mathrm{S}$, the decay is faster and even exceeds $S^{3}$.

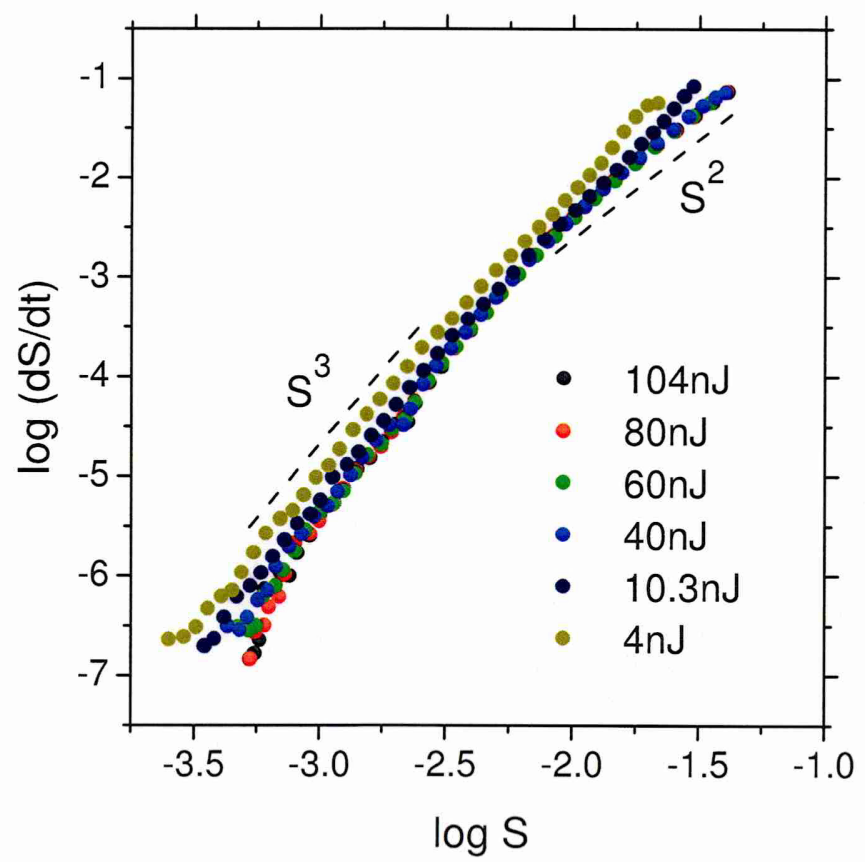

Figure 5-5: The derivative of $S=\Delta T / T_{0}$ as function of $S$ for ss-DNA wrapped carbon nanotubes sample, dashed lines indicate the region of $S^{2}$ and $S^{3}$.

The data from Fig. 5-5 suggests (at least at the highest excitation levels) a transition 
(crossover) between two power law decays observed at early and long time. This can be seen clearly by plotting the reaction order $\beta$ where $d n / d t=-k n^{\beta} . \beta$ is the logarithmic derivative of Fig. 5-5 and can be calculated from the experimental signal $S$ as follows

$$
\beta=\frac{d \ln (d S / d t)}{d \ln S}=S \frac{d^{2} S / d t^{2}}{(d S / d t)^{2}}=n \frac{d^{2} n / d t^{2}}{(d n / d t)^{2}}
$$

assuming that the measured differential transmission is proportional to the exciton density $n$. Hence $\beta$ (like $\alpha$ ) is independent of the proportionality constant.

The reaction order $\beta$ for each high pump intensity is plotted in Fig. 5-6. This figure shows a distinct region of $\beta \sim 2$ for times between $\sim 0.5$ and $\sim 1 \mathrm{ps}$, corresponding to a rate equation $d n / d t \propto n^{2}$. Similarly at times between $\sim 4$ and $\sim 10$ ps there is another distinct region of $\beta \sim 3$ corresponding to $d n / d t \propto n^{3}$. At times $<0.5 \mathrm{ps}$, the value of $\beta$ drops off rapidly and become negative; these early dynamics are discussed further in chapter 7 . At long time $(t>10 \mathrm{ps}$ ), the value of $\beta$ exceeds 3 and it will be discussed in detail in subsection 5.3.2. The crossover between $\beta=2$ and $\beta=3$ will also be further discussed in section 5.3.2.

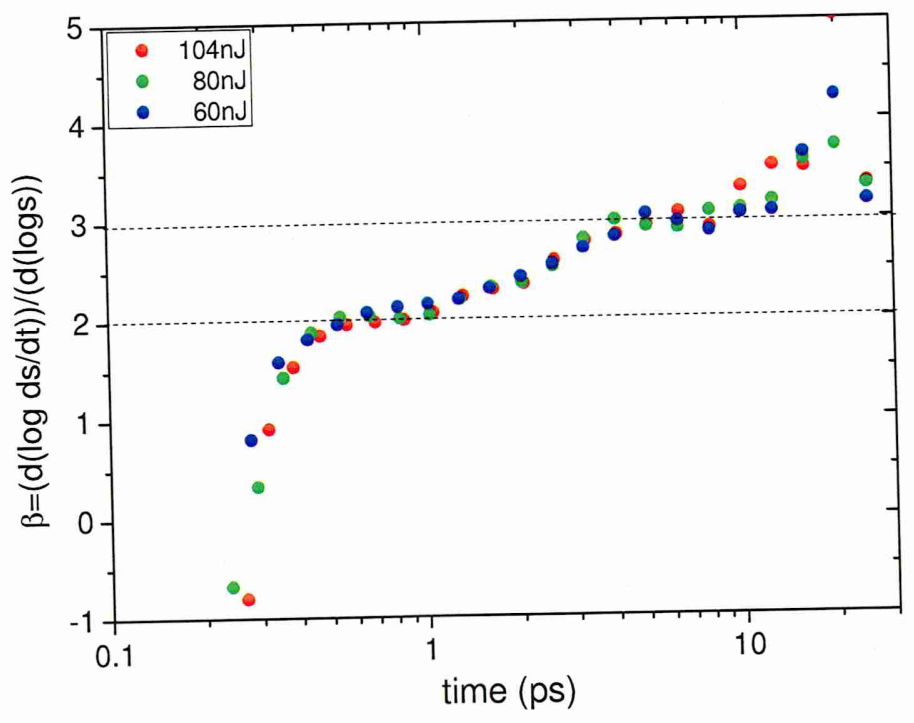

Figure 5-6: Second logarithmic derivative ' $\beta$ ' of pump probe data plotted only for highest pump fluences up to $30 \mathrm{ps}$, clearly show the transition between two power law behaviours.

In summary, in this section we presented experimental measurements of exciton decay in solution of semiconducting HiPco SWNTs. By examining the underlying rate equation 
directly from experimental data, we observed (for the highest excitation density used) distinct regions of reaction-limited behaviour where $d n / d t \propto n^{2}$ and diffusion-limited behaviour where $d n / d t \propto n^{3}$. However a strict $t^{-1}$ decay of the exciton population in the reactionlimited regime was not observed due to the effect of the constant of integration. At long and short times the measurements deviate from these power laws. The behaviour also deviates as the excitation density decreases, and these will be further discussed in next section.

\subsection{Direct Determination of the Exciton Population}

According to theoretical predictions summarised in chapter 2, both the exponent and amplitude of the asymptotic $t^{-1 / 2}$ power law decay should be universal, i.e. independent of initial exciton density. However it can be seen in Fig. 5-3 that the curves at low excitation are displaced. We assume that this is due to the combined effects of non-uniform excitation and absorption saturation and will justify later. It can also be seen from Fig. 5-3 that a deviation from $t^{-1 / 2}$ power law decay was seen at delay times approaching $\sim 1 \mathrm{~ns}$, and that this is dependent on excitation density. We assume that this reduction in reaction rate at long time occurs when bimolecular reaction cease i.e. when the population approaches one exciton per nanotube.

To fit this regime we use Simon's equation for the bimolecular coalescence reaction in a 1D system of finite length given in equation 2.18. The two fitted parameters for each excitation density are the diffusion coefficient and a scaling parameter related to the number of nanotubes excited, the absorption coefficient, the concentration of nanotubes, and the pump focussing geometry. This removes the need to estimate these parameters. The regions fitted are shown in Fig. 5-7. It will be shown below (e.g in Fig. 5-10) that even at low excitation where the deviation from a power law is small, it is still sufficient to determine the scaling and diffusion parameters accurately. Hence, if the point $\left(N=1, t=L^{2} /(2 \pi D)\right)$ can be identified experimentally, it allows calibration of both the exciton number $N$ as well as intrinsic timescale $D t$, without any approximation or dependence on any other parameter other than the measured length of the nanotubes. As our sample showed a significant variation of nanotube length, the fitting was performed assuming an ensemble of nanotubes with the length distribution as shown in Fig. 4-8.

Note that a small correction has been applied to the data in Fig. 5-7. A small secondary signal appeared at approximately 20 ps in the data (e.g. see Fig. 5-3) due to a weak reflection 
in the optical path. This is hardly visible in the raw data but becomes significant when the data is differentiated twice. In order to subtract it, a $t^{-1 / 2}$ baseline was determined by fitting the data at $10 \mathrm{ps}$ and $100 \mathrm{ps}$, and then the deviation from this line in the vicinity of $20 \mathrm{ps}$ was fitted by an appropriate power law. The omission or inclusion of this correction does not change the qualitative conclusions made from the data.

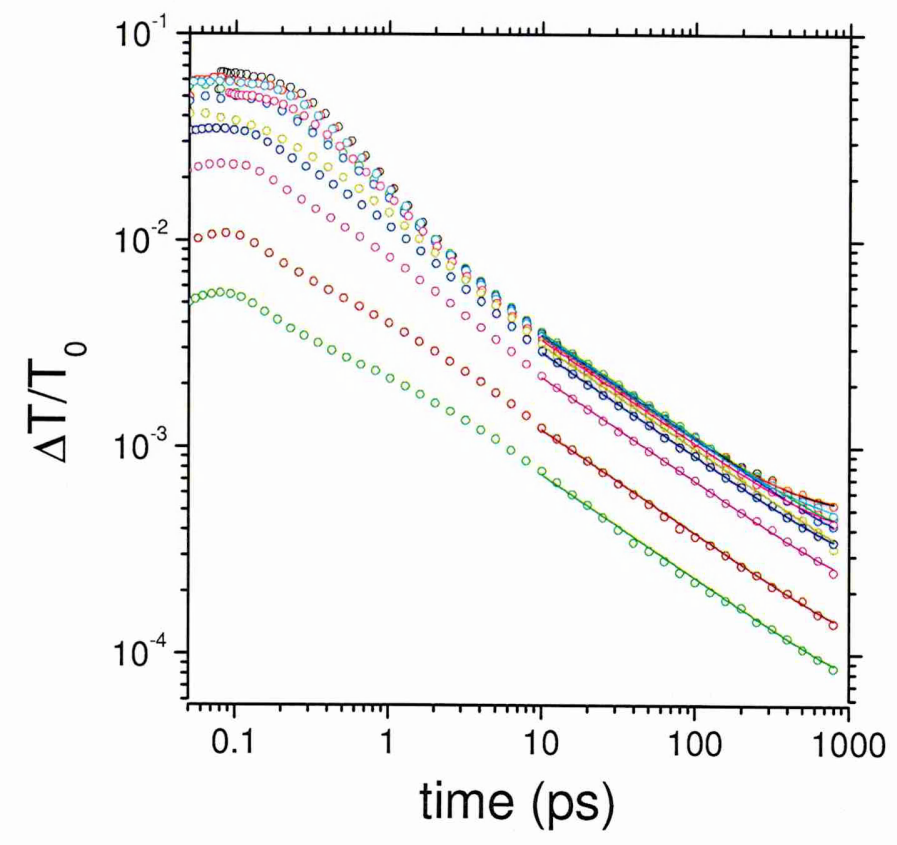

Figure 5-7: Differential transmission as function of pump probe delay for the pump powers between $10 \mathrm{uW}$ to $26 \mathrm{~mW}$. The solid lines are fits with Simon's formula.

Having obtained the scaling factor for different excitation densities, we plot the maximum number of excitons created per nanotube as a function of pump power in Fig. 5-8 (a). This figure shows that the exciton population initially increases with optical power and saturates after $P_{0}=3 \mathrm{~mW}$. This saturation of exciton population suggests that there is limit on the maximum number of excitons (145 \pm 8 excitons per nanotube) which can accommodate on a single nanotube. The average length of nanotubes measured with AFM is $(184 \pm 5) \mathrm{nm}$ which corresponds to an exciton density of $n_{0}=\frac{N_{0}}{L}=(0.79 \pm 0.05)$ excitons per nm and exciton separation of $(1.26 \pm 0.08) \mathrm{nm}$. This minimum exciton separation is a measure of the exciton size which can be compared to the FWHM of wave function of exciton.

This absorption saturation when the exciton spacing equals the exciton size has been previously attributed to Pauli blocking by Lüer et al. [13]. Their reported value for exciton 
size was $(2.0 \pm 0.7) \mathrm{nm}$ which is in agreement with our value, although less precise. Our finding of exciton size is also in good agreement with Capaz et al. [14] who reported exciton size in the range of $1.3-1.6 \mathrm{~nm}$ for nanotubes with diameter $0.9 \mathrm{~nm}$ using a variational approach utilising a tight binding model. They define the exciton size as width of a Gaussian exciton wave function. Hence the saturation observed at high intensities in our experimental data is direct measurement of absorption saturation due to Pauli exclusion between the fermionic constituents of the excitons.
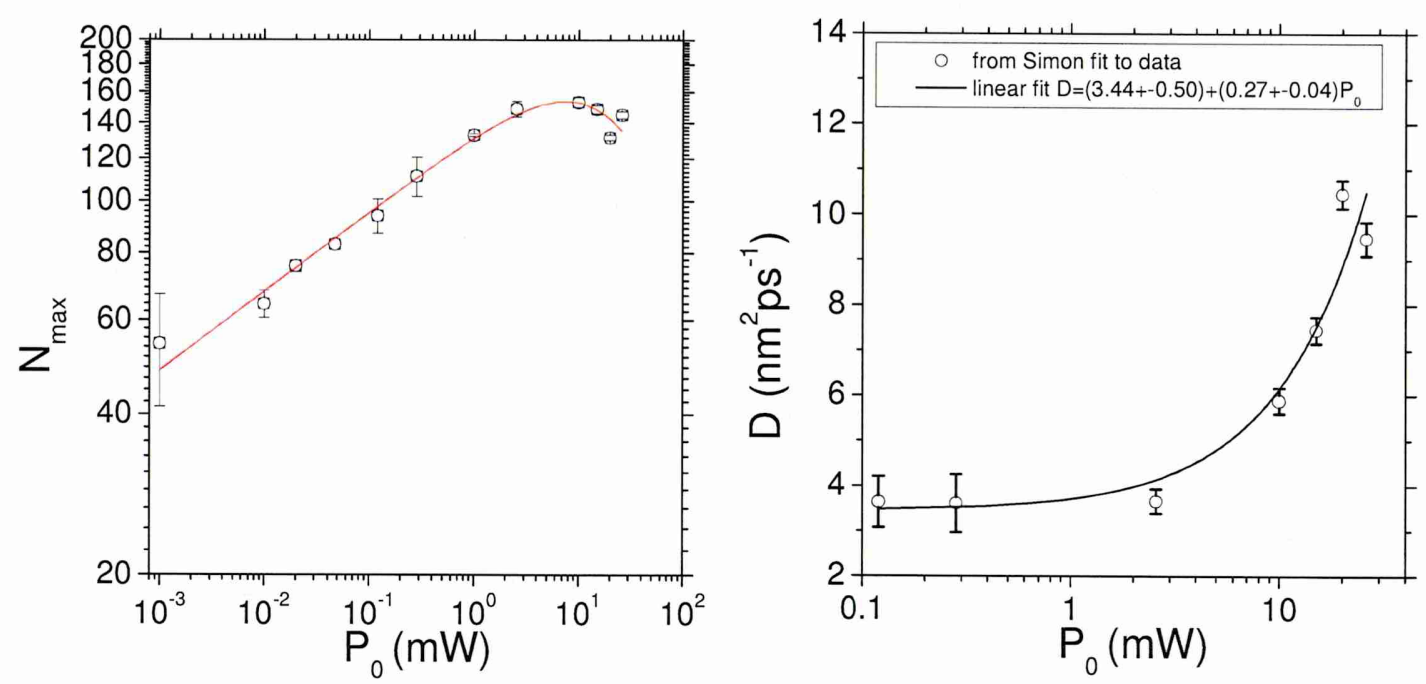

Figure 5-8: (a) Maximum number of excitons created per nanotube, as a function of laser power. The exciton density saturates at 145 excitons per nanotube due to Pauli Exclusion principle. (b) The fitted value of diffusion coefficient D obtained from figure 5-7 plotted as function of laser power. Circles are values of diffusion coefficient D obtained from Simon's formula and solid line is a linear fit.

According to exclusion principle, the transitions into occupied states are forbidden. It corresponds to the well known band filling effects in the case of generation of free carriers. However, for the case of generation of excitons, the effect due to exclusion principle is less known [123]. As exciton is best described as composite fermions (details are described in [79]) i.e. built up from a linear combination of single-particle fermions states distributed according to $\psi_{e x}(k)$, the Fourier transform of the exciton relative motion orbital wavefunction $\psi_{e x}(x)$. Hence same theory of exclusion principle can be used for excitons as well. The resonant generation of excitons corresponds to a distribution of electron $\left(f_{e}(k)\right)$ and hole $\left(f_{h}(k)\right)$ oscillator strengths which is related to $\psi_{e x}(k)$ by [123] 


$$
f_{e}(k)=f_{h}(k)=\frac{n}{2}\left|\psi_{e x}(k)\right|^{2}
$$

where $n$ is excitons density per unit length. Hence upon the creation of one exciton, there is an overall reduction of escillator strength due to exclusion principle through "phase space filling" effect [123]

$$
\frac{\delta f}{f}=-\sum_{k}\left[f_{e}(k)+f_{h}(k)\right] \frac{\psi_{e x}(k)}{\psi_{e x}(x=0)}
$$

In the case of one-dimensional exciton, the relative motion exciton wavefunction can be approximated by a Gaussian wavefunction using tight-binding model [14]

$$
\psi_{e x}(x)=\frac{1}{\sqrt{a_{e x} \sqrt{\pi}}} e^{-x^{2} /\left(2 a_{e x}^{2}\right)}
$$

where $a_{e x}$ is the electron-hole correlation length (exciton size). Then equation 5.3 becomes

$$
\frac{\delta f}{f} \sim-\frac{n}{n_{s}}
$$

where $n_{s} \propto \frac{1}{a_{e x}}$. Experimentally, the reduction in oscillator strength is equal to relative transient ground-state bleaching i.e. $\frac{\delta f}{f}=-\frac{\Delta A}{A}$, this was the expression which Lüer [13] used to relate the absorption saturation to oscillator strength and determine the exciton size. Using a Gaussian wavefunction in equation 5.4 , the equation 5.2 becomes

$$
f_{e}(k)=f_{h}(k)=N a_{e x} \sqrt{\pi} * e^{-k^{2} a_{e x}^{2}}
$$

This is the density of wavevector states. The density of energy states can be calculated using $E=\frac{\hbar^{2} k^{2}}{2 m^{*}}$ and it becomes

$$
f_{e}(E)=f_{h}(E)=N a_{e x} \times \sqrt{\frac{\pi m^{*}}{2 \hbar^{2} E}} \times e^{\left(-\frac{2 m^{*} a_{e x}^{2} E}{\hbar^{2}}\right)}
$$

where $m^{*}$ is reduced electon-hole mass and is given by $m^{*}=0.05 m_{0}$ [2]. The expected value of energy can be calculated via

$$
\bar{E}=\int_{0}^{\infty} N a_{e x} \sqrt{\frac{\pi m^{*} E}{2 \hbar^{2}}} \times e^{\left(\frac{-2 m^{*} a_{e x}^{2} E}{\hbar^{2}}\right)} d E
$$




$$
\bar{E}=\frac{\pi}{8} \times \frac{N \hbar^{2}}{m^{*} a_{e x}^{2}}
$$

The expected value of $0.35 \mathrm{eV}$ is calculated using above expression. This value of Fermi energy is very close to reported value of exciton binding energy (discussed in section 2.1.4). This shows that at high densities, length scale becomes comparable to exciton binding energy. Mott dissociation is expected to occur as it occurs in 2D and 3D semiconductors. However, the spectrally resolved data given in chapter 7 shows that excitons remain intact at high densities and there is no evidence of Mott dissociation.

Also from fitting of the experimental data with Simon's equation, we extracted different values of the diffusion coefficient $D$ that varied from $D=(3.6 \pm 0.6) n m^{2} \cdot p s^{-1}$ to $D=(10.4 \pm 0.3) \mathrm{nm}^{2} \cdot \mathrm{ps}^{-1}$ depending on the excitation power. The variation of diffusion coefficient $D$ with excitation power may be due to photoinduced heating of the nanotubes. This was tested by experiments in which the laser repetition rate was varied while the pulse energy was fixed: these are briefly discussed in Chapter 7. The diffusion coefficient $D$ is plotted in Fig. 5-8 (b) which shows a linear variation with pump power. We discuss further the values of diffusion coefficient in a following section.

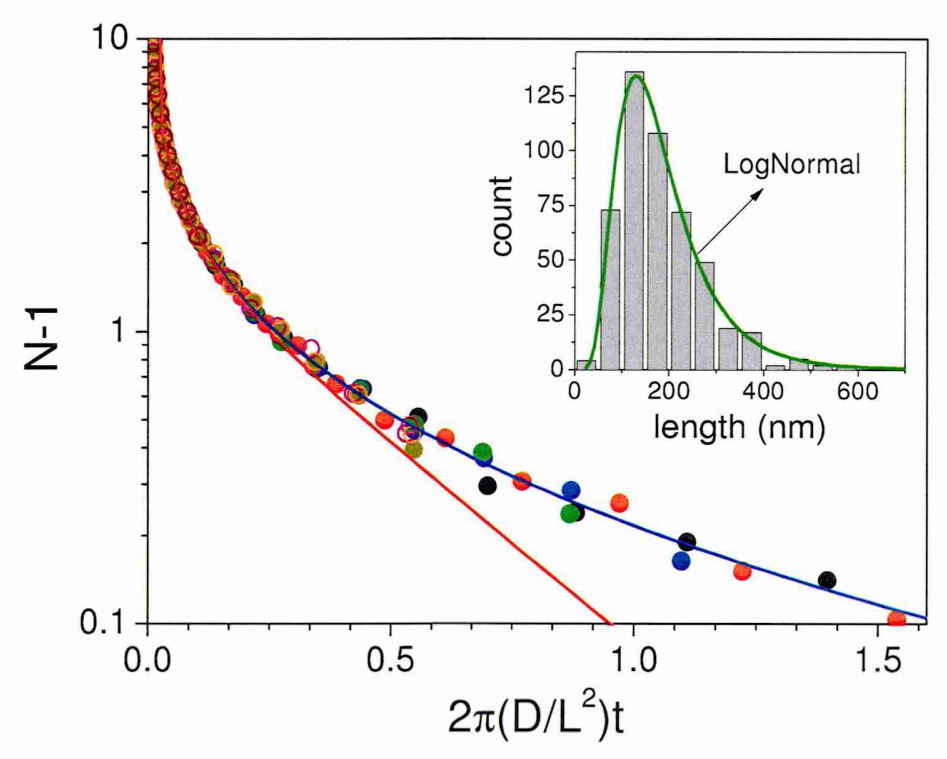

Figure 5-9: N-1 as function of scaled time, where the parameters are determined from the fitting of experimental data (from figure 5-7) with Simon's equation 2.18. The red line is fit with Simon's equation using average nanotube length of $184 \mathrm{~nm}$. The blue line shows Simons equation convolved with the length distribution. The inset shows the distribution of nanotubes lengths measured with AFM fitted with LogNormal distribution. 
The transition from many excitons to few excitons per nanotube is investigated by plotting the logarithm of $N-1$ against linear time (rescaled to account for variations of diffusion coefficient) in Fig. 5-9. Symbols are experimental data for the different excitation densities, and the behaviour is found to be independent of excitation density in this regime. The red line is a fit using Simon's equation (given in eq. 2.18) with the average nanotube length of $184 \mathrm{~nm}$ and the blue line is a fit obtained using Simon's equation convolved with the distribution of nanotube length which fitted very well with the experimental data. Inset shows the distribution of nanotubes length measured with AFM. The approximate straight line (linear behaviour) at long times indicates that the single exciton limit is approached exponentially. In the language of critical systems, this is a "finite size effect", and occurs because the scale invariant power law decay gives way to an exponential decay which is characterised by the time required for a exciton to diffuse across the finite length of the nanotube.

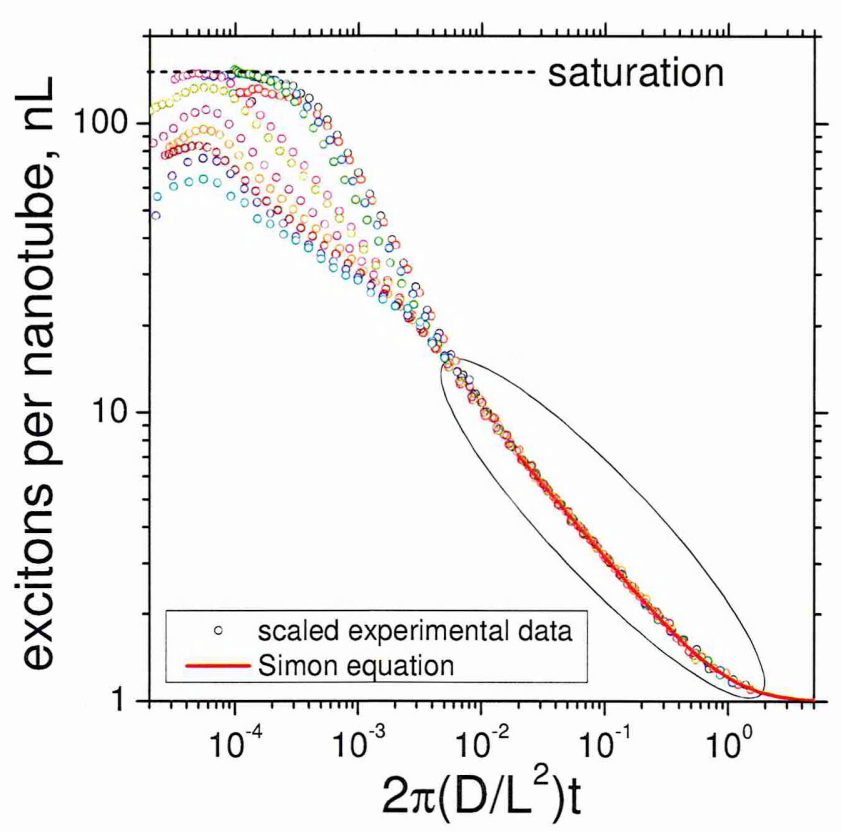

Figure 5-10: Exciton number as a function of scaled time. The scaling is performed based on the fitted parameters of the Simon equation 2.18. The circled region shows universal behaviour, independent of excitation density.

In Fig. 5-10, we plot the average number of excitons created per nanotube $(N=n L)$ for each pump power as a function of scaled time with parameters (diffusion coefficient $D$ ) obtained from fitting of Fig. 5-7. This figure shows that the exciton population is independent 
of initial population at long times, and all the data points collapse onto a common curve. This is the characteristic signature of universal behaviour and is well described by Simon's formula (e.g. red line in the circle). This is one of the significant results of this work since although universality of the exponent and amplitude is one of the key characteristics of this reaction-diffusion system; it does not appear to have been previously demonstrated by experiment.

Four different regions can be easily distinguished from this figure: (1) saturation of exciton density at early time, (2) a region in the intermediate time where exciton population follows a power law with positive deviation from $t^{-1 / 2}$ at high densities and negative deviation at low densities, (3) a region where exciton population follows universal $t^{-1 / 2}$ power law and (4) the deviation from the $t^{-1 / 2}$ power law around $\sim 1 \mathrm{~ns}$, where there is only one exciton per nanotube.

The observed universal asymptotic $t^{-1 / 2}$ power law decay can be explained by the diffusion-limited single species two body coalescence (or fusion) process in a 1D system [71] and is governed by rate equation 2.15. Our results for ss-DNA wrapped HiPco SWNTs are consistent with a previous report by Russo et al. [71] for surfactant-aided suspensions of HiPco SWNTs where the exciton dynamics follow a $t^{-1 / 2}$ power law. The similarity between our results and those reported in [71] provides evidence that the decay mechanism is qualitatively independent of the environment in which nanotubes are being held.

Note that Toussaint and Wilczek [57] reported a $t^{-1 / 4}$ power law for diffusion-limited reactions between two non-identical particles (e.g. an uncorrelated electron and hole). Hence our results are consistent with the formation of stable excitons in SWNTs due to strong Coulombic interactions.

Fast decay observed at early time (in the case of high optical densities) which shows positive deviation from $t^{-1 / 2}$ power law is signature of reaction-limited exciton-exciton annihilation in which intrinsic annihilation rate is limiting factor in the decay of exciton population. This means that at high intensity and shorter time delays, the population of excitons is so high that negligible transport is required before the annihilation process to occur. Therefore this regime is limited by reaction time rather than diffusion time and a mean field treatment of two-body reaction kinetics (given in equation 2.12) predicts a population relaxation of the exciton density in this regime. The slow decay at early time (in the case of low excitation densities) is due to non-uniform excitation of nanotubes. 


\subsection{Crossovers and Long range Interaction}

In this section of the chapter, we discuss the dynamical crossover from reaction-limited to diffusion-limited behaviours and long range interaction of excitons on carbon nanotubes. The analysis in this section only considers data at the highest excitation intensity. This section is arranged in such a way that brief description of asymptotic behaviours observed at high and low populations is given in subsection 5.3.1, where the reaction coefficient and diffusion coefficient are deduced from the fitting of experimental data with asymptotic rate equations. In section 5.3.2, we described the crossover between reaction-limited and diffusion-limited regimes in detail and discuss reaction-diffusion process in terms of short range and long range interaction of excitons. In the same section, we developed a simple new model to incorporate long range interaction and compare it with that of ZBA [54] lattice based analytical expression for short range interaction.

\subsubsection{Asymptotic Behaviours and Rate Equations}

In Fig. 5-11, we plot the experimental data for $d N / d t$ as function of $N$ for the case of the highest excitation power $(26 \mathrm{~mW})$.

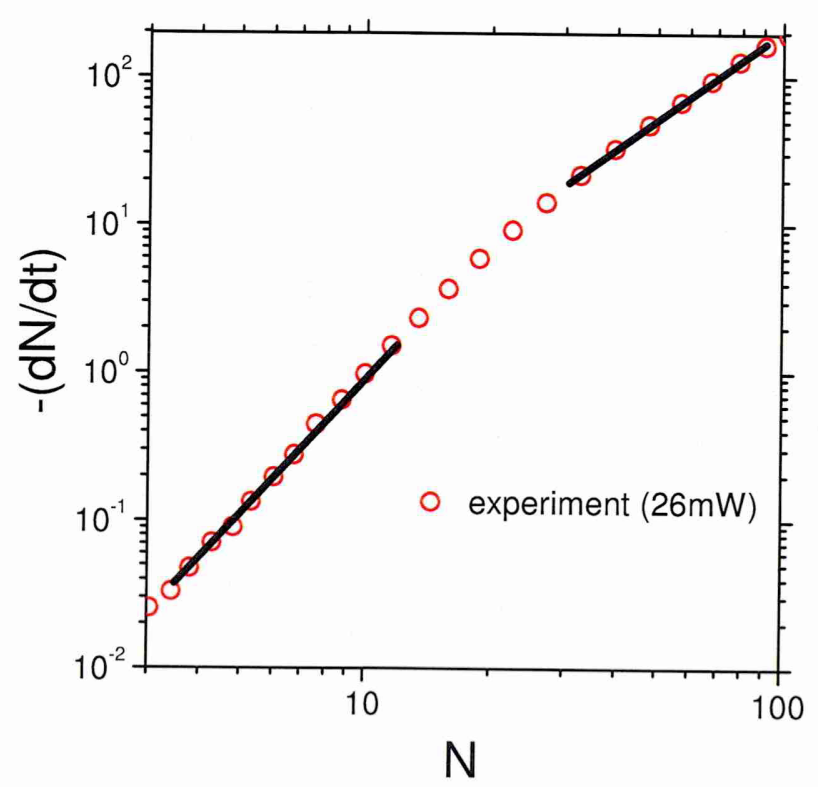

Figure 5-11: Reaction rate is plotted against the exciton number N. The black lines are asymptotes of reaction-limited and diffusion-limited rate equations, included here as guide to eye. 
In this figure, we neglect the data for $\mathrm{N}<2.5$ where the exponential to the single exciton state sets in, and also neglect data for $\mathrm{N}>100$ where there are deviations from power law behaviour at very short times. The figure shows that there are clearly two distinct regimes at high and low populations. The solid black lines in the figure represent $d N / d t \propto N^{2}$ and $d N / d t \propto N^{3}$, and fit the asymptotic behaviours very well. The $N^{2}$ dependence of experimental data at high concentrations is proof of the existence of a reaction-limited (mean-field) regime (described by equation 2.12). The reaction rate constant $k_{r}=(3.76 \pm 0.04) n m \cdot p s^{-1}$ and diffusion coefficient $D=(8.1 \pm 0.4) n m^{2} \cdot p s^{-1}$ are deduced from these asymptotic fits.

\subsubsection{Reaction-Diffusion Crossover and the Interaction Range}

In chapter 2, we described the existence of reaction-limited and diffusion-limited regimes and their characteristic exponents in 1D bimolecular coalescence (fusion) reactions. We also discussed the theoretical treatment of the crossover between these regimes, based on a Monte Carlo simulation [56], an analytical scaling theory [64], and an approximate 'empty interval' approach [54], and experimental observation of a crossover between reaction- and diffusionlimited regimes in the decay of excitons on SWNTs by Sutton [74].

In the previous section, we showed the existence of the asymptotic regions and determined asymptotic reaction coefficients. In this section, we consider the crossover between these regions in more detail. First a new equation to describe this crossover was derived, which is mathematically equivalent to the H\&M $[64,65]$ equation. (The original derivation of this equation and its later modification for long-range interactions and sub-diffusive transport was performed by J. Allam. This theory was used to model the data throughout this thesis and so is described in detail here). We use this equation to fit experimental data for the decay of exciton number (presented above in section 5.2). The crossover is not described well by this equation. We therefore modify the equation to include a long-range interaction. This is found to fit the data remarkably well for plausible interaction ranges.

The reaction rate for a bimolecular reaction process $\mathrm{A}+\mathrm{A}$ can be written as

$$
\frac{d n}{d t}=-k n^{2} .
$$


Hynes and Northrup [124, 125] showed that the overall reaction coefficient for a sequential two-step reaction is given by

$$
\frac{1}{k}=\frac{1}{k_{1}}+\frac{1}{k_{2}}
$$

where $k_{1}$ and $k_{2}$ are the reaction coefficients associated with steps 1 and 2 respectively. This is equivalent to summing the incremental time associated with each step as follows:

$$
\frac{d t}{d n}=-\frac{1}{k n^{2}}=-\frac{1}{k_{1} n^{2}}-\frac{1}{k_{2} n^{2}}=\left(\frac{d t}{d n}\right)_{1}+\left(\frac{d t}{d n}\right)_{2}
$$

For the reaction-diffusion system, the coefficients are $k_{1}=k_{d} n=\pi D n$ for the diffusion step (where the dependence on $n$ gives rise to the anomalous reaction rate) and $k_{2}=k_{r}$ for the reaction step. Hence the (inverted) rate equation is

$$
\frac{d t}{d n}=-\frac{1}{k_{d} n^{3}}-\frac{1}{k_{r} n^{2}}=\left(\frac{d t}{d n}\right)_{d}+\left(\frac{d t}{d n}\right)_{r}
$$

This equation can be easily integrated to give

$$
t(n)=\frac{1}{2 k_{d}}\left(\frac{1}{n^{2}}-\frac{1}{n_{o}^{2}}\right)+\frac{1}{k_{r}}\left(\frac{1}{n}-\frac{1}{n_{o}}\right)=t_{D}(n)+t_{R}(n)
$$

i.e. the total time taken to reach a concentration $n$ from initial density $n_{0}$ is simply the sum of diffusion time $t_{D}(n)$ - the time taken by the particles to find each other - and the reaction time $t_{R}(n)$ - the time taken by them to react once they are in contact.

This expression can be further rearranged to get

$$
t(n)=t_{0}\left(\frac{n_{0}^{2}}{n^{2}}-1\right)+t_{1}\left(\frac{n_{0}}{n}-1\right)
$$

where $t_{0}=1 /\left(2 k_{d} n_{0}^{2}\right)$ is the average time for the excitons to encounter each other and $t_{1}=1 /\left(k_{r} n_{0}\right)$ is the average time for a reaction to occur. We also define $t_{2}$ as the time when the diffusion time and the reaction time are equal. In the asymptotic regime where $n \ll n_{0}$ or 
$t \gg\left(t_{0}+t_{1}\right)$, this is found out to be $t_{2}=2 k_{d} / k_{r}^{2}$ which occurs at $n_{2}=k_{r} / 2 k_{d}$.

Equation 5.9 can be inverted to give the decay dynamics

$$
n(t)=\frac{n_{o}}{2} \frac{1+\sqrt{\left(1+\frac{k_{r}}{k_{d} n_{o}}\right)^{2}+2 \frac{k_{r}^{2}}{k_{d}} t}}{1+\frac{k_{r}}{2 k_{d} n_{o}}+n_{o} k_{r} t}
$$

This equation 5.11, expressed purely in terms of macroscopic variables, is mathematically equivalent to the H\&M $[64,65]$ equation 2.19 where the diffusion process is described on a lattice of spacing $\Delta z$ with hopping time $t_{h o p}=(\Delta z)^{2} / 2 D$. The concentration per lattice site is $\rho=n \Delta z$ and the normalised timescale is $\tau=t / t_{\text {hop }}$. The crossover in the reaction rate occurs at $n=n_{c}=k_{r} / k_{d}$. Reactions are assumed to occur with probability $p$ when two particles co-exist on the same lattice site.

The equivalence of equation 5.11 and H\&M equation 2.19 indicates that the form chosen by $H \& M$ is consistent with separable reaction and diffusion processes, and also that these equations are supported by simple Monte Carlo simulations.

On other hand, ZBA [54] derived a very similar relation for the same 1D reactioncoalescence problem (given in equation 2.20), using a completely different theoretical approach, based on the inter particle distribution function (IPDF) [55]. The corresponding ZBA rate equation (in macroscopic variables $n, t$ ) is

$$
\frac{d n}{d t}=-k_{d} \frac{n^{3}}{1+(1-p)\left(k_{d} / k_{r}\right) n}
$$

The asymptotic behaviour of this equation at high concentration is

$$
\frac{d n}{d t}=-\frac{k_{r}}{1-p} n^{2}=-k_{r}^{\prime} n^{2}
$$

The value of $k_{r}$ in ZBA equation is modified as compared to the H\&M equation. We used equations $5.8(\mathrm{H} \& \mathrm{M})$ and $5.12(\mathrm{ZBA})$ to fit the experimental data shown in Fig. 5-12 (blue and orange lines respectively), using the previously determined values of $k_{r}^{\prime}=3.76 \mathrm{~nm} \mathrm{ps}^{-1}$ and $k_{d}=\pi \times 8.1 \mathrm{~nm}^{2} \mathrm{ps}^{-1}$ in order to make sure that the asymptotic 
behaviour is reproduced. The Fig. 5-12 shows that that crossover between reaction-limited and diffusion-limited regimes occurs more abruptly in the experiment than is predicted by the ZBA (or H\&M) theories.

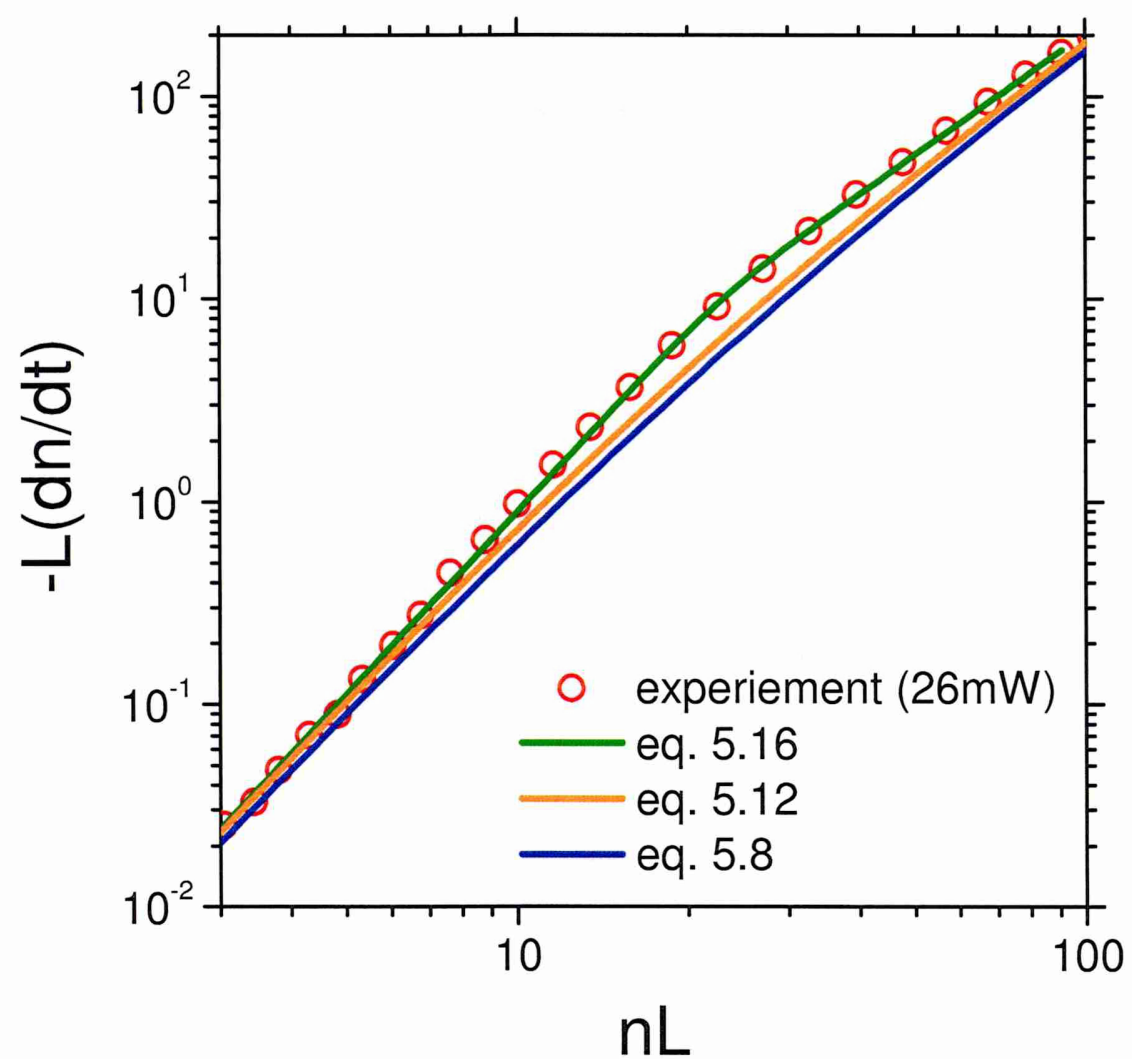

Figure 5-12: Dynamic crossover between reaction-limited and diffusion-limited regimes. Reaction rate is plotted against exciton number, on a log-log scale. Blue line is conventional theory with short-range interactions. Green line is new theory with interaction range of $7 \mathrm{~nm}$.

The crossover between reaction-limited and diffusion-limited regimes can be examined in greater detail by calculating the logarithmic derivative of Fig. 5-12. This is equal to the reaction order $\beta$ which can be calculated from the mathematical relation of equation 5.1. An expressions for $\beta$ obtained using equation 5.1 and equation 5.8 is given by

$$
\beta=2+\frac{1}{1+\frac{k_{d}}{k_{r}} n}
$$

where $k_{r}^{\prime}=k_{r}$ in the H\&M version and $k_{r}^{\prime}=\frac{k_{r}}{1-p}$ in the ZBA version. This equation is 
plotted in Fig. 5-14 (green line) which shows that $\beta$ varies gradually and monotonically between the asymptotic values of $\beta=2$ and $\beta=3$ and requires approximately a two orders of magnitude change in population for a $10 \%-90 \%$ transition. The experimental values for the reaction order which were obtained by twice differentiating the experimental data according to equation 5.1 are superimposed on Fig. 5-14 (red symbols). Experimentally the transition is clearly more abrupt than predicted by conventional theory and is also nonmonotonic i.e. exceeding the upper asymptotic value of $\beta=3$.

This non-monotonic crossovers between regions of mean-field and critical behaviour have been observed previously in several classes of equilibrium phase transitions [126-128] and in each case, non-monotonic behaviour was related to a long range interaction between particles.

A simple way of including a long-range interaction into the description of a reactiondiffusion process is to reduce the distance which a particle must diffuse before it can react with a neighbouring particle. For the case of zero-range interactions, this is just the interparticle separation $x$ and the time taken for these particles to meet is $x^{2} / 4 D$, whereas for a finite reaction range $r$ and for particle separations $x \gg r$, the time taken for a particle pair to diffuse until they come close to each other within the reaction range is modified to $t=(x-r)^{2} / 4 D$ (shown in Figure 5-13). If we use the same approximation as used in equation 5.9 i.e. the incremental time for an ensemble of particles of density $n$ to react is the sum of this diffusion time and a reaction time, then we obtain

$$
-\frac{d t}{d n}=\frac{1}{n} \frac{\overline{(x-r)^{2}}}{4 D}+\frac{1}{k_{r} n^{2}}=\frac{1}{n} \frac{\overline{x^{2}}}{4 D}-\frac{1}{n^{2}} \frac{r}{2 D}+\frac{1}{n} \frac{r^{2}}{4 D}+\frac{1}{k_{r} n^{2}}
$$

where $\bar{x}=\frac{1}{n}$. In the asymptotic limit, $r$ is negligible compared to the particle separation and so for consistency with exact solutions in this limit we take $\overline{x^{2}}=\frac{4}{\pi} \bar{x}^{2}=\frac{4}{\pi n^{2}}$. However we note that the quantity $\overline{x^{2}} / \bar{x}^{2}$ which characterises the interparticle distribution function will be different in the reaction-limited regime and will be further modified by the presence of long-range interactions. This is proposed as a topic of future research in chapter 7.

At much higher concentrations i.e. when particle separations is $x \ll r$, the diffusion time is negligible compared to the reaction time (reaction-limited regime). We therefore construct a piecewise solution which is expressed in terms of the rate coefficients $k_{r}$ and $k_{d}=\pi D$ in 
order to approximate the main effect of the long-range interaction:

$$
\begin{array}{cc}
-\frac{d t}{d n}=\frac{1}{\pi D n^{3}}+\frac{1}{k_{r} n^{2}}\left(1-\frac{\pi}{2} \frac{k_{r}}{k_{d}} r\right)+\frac{1}{n} \frac{r^{2}}{4 D} & (n r \ll 1) \\
-\frac{d t}{d n}=\frac{1}{k_{r} n^{2}} & (n r \gg 1)
\end{array}
$$

Although this model is very simple but it can reproduce the main features of the experimental data (green line shown in Fig. 5-12), and is also consistent with Monte Carlo simulations performed by J. Allam and T. Amoah (reported elsewhere).
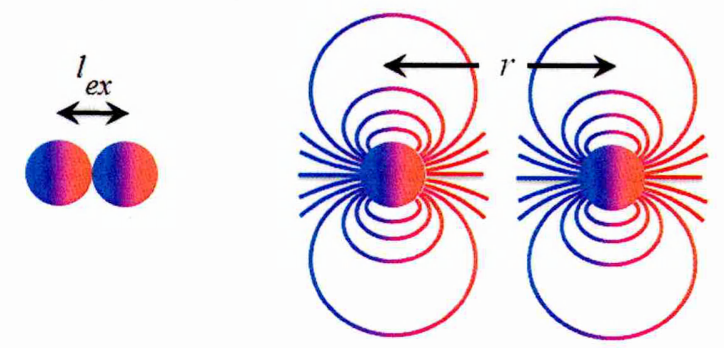

Figure 5-13: Schematic of short range interaction and long range (dipole-dipole) interaction. $L_{e x}$ is exciton length and $r$ is interaction range.

The above approach sheds light on the ZBA expression, equation 5.12. ZBA studied the reaction-diffusion system on a lattice with spacing $\Delta z$ where more than one particle was forbidden to occupy same lattice site, so that the effective reaction range was $r=\Delta z$. With $k_{r} / k_{d}=2 p /(\pi \Delta z)$ the equation 5.16 a becomes

$$
-\frac{d t}{d n}=\frac{1}{\pi D n^{3}}+\frac{1}{k_{r} n^{2}}(1-p)+\frac{1}{n} \frac{(\Delta z)^{2}}{4 D} \quad(n<1 / \Delta z)
$$

If we neglect the final term which is only important when the lattice is close to full $(n \Delta z$ approaches 1), then above expression (eq. 5.17) is identical to that of ZBA (eq. 5.12) but it differs from that of $H \& M$ by the factor $(1-p)$ when expressed in the same parameters. Hence it is shown that the assumptions present in the H\&M and ZBA approaches are both equivalent to the assumption that the diffusion and reaction stages are sequential (equation 5.16), and further that the difference between the two approaches arises from the finite 
(small) interaction range which appears in the ZBA approach. Our equations 5.16a and 5.16b therefore represent a generalisation of the ZBA equations where the reaction range is decoupled from the exclusion length and the hopping range.

For long range interaction, the reaction order $\beta$ can be obtained by differentiating equation 5.16

$$
\begin{array}{ll}
\beta=2+\frac{1-\frac{\pi}{4} r^{2} n^{2}}{1+n \frac{k_{d}}{k_{r}}\left(1-\frac{\pi}{2} \frac{k_{r}}{k_{d}} r\right)+\frac{\pi}{4} r^{2} n^{2}} & (r n<\sqrt{4 / \pi}) \\
\beta=2 & (r n>\sqrt{4 / \pi})
\end{array}
$$

The boundary between the two regions is chosen so that beta is continuous at the boundary. Equation 5.18a shows that there is a maximum value of beta which occurs at $n r \sim 2 / \pi$ and is approximately $\beta \sim 4$. Thus in the case of long range interactions, the reaction order $\beta$ can take values above 3 as observed in the experimental data. This expression (given in equation 5.18) of reaction order $\beta$ is used to fit the experimental data as shown in the Fig. 5 -14. In this figure, the experimental data is the average of the highest three pump powers (26 $\mathrm{mW}, 20 \mathrm{~mW}, 15 \mathrm{~mW}$ ), which behave quite similarly in the crossover region. This was done to improve the signal-to-noise ratio in the data, and the errors bars represent the standard error of the averaging process. For comparison, the reaction order obtained from ZBA model of short-range interaction (green line, calculated using the same value of $k_{r} / k_{d}$ as the longrange model) is also shown.

It is clear from Fig. 5-14 that the transition is more abrupt and value of $\beta$ exceeds 3 experimentally and cannot be explained by the ZBA model of short range interactions which shows a gradual transition (green line), and also shows that value of $\beta$ cannot be more than 3 . Our model incorporating a long range interaction (blue line) fits the data remarkably well considering the simplicity of the model. The parameters obtained from the fit are the crossover concentration $n_{c}=\frac{k_{r}}{k_{d}}=(0.19 \pm 0.02) \mathrm{nm}^{-1}$ and the reaction range $r=(6.8 \pm 0.3) n m$. From $n_{c}$, the reaction probability is $p=k_{r} \Delta z /(2 D)=0.38 \pm 0.02$ which is greater than the overlap integral between two Gaussian wave packets separated by their FWHM. This provides further evidence that the exciton-exciton interaction cannot be explained by short-range exchange interactions. The reaction probability distributed across the lattice sites within the reaction range $r$ is $\sim 0.07$ per lattice site. The reaction range of 
$\sim 7 \mathrm{~nm}$ is within the range of values for which Forster energy transfer between nanotubes has been reported [129-132], where the dipole-dipole interaction can extend over a range up to $\sim 10 \mathrm{~nm}$.

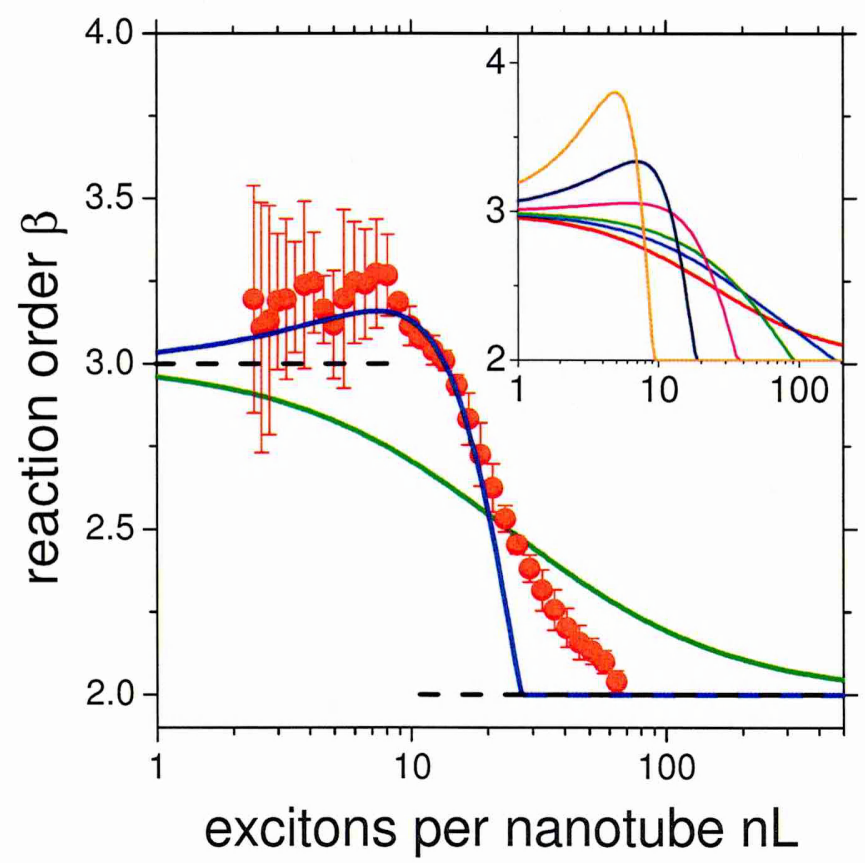

Figure 5-14: Reaction order $\beta$ plotted as function of exciton number. Experimentally, transition (from $\beta=2$ to $\beta \sim 3$ ) is much more abrupt than predicted by conventional theory (green curve). The blue line is the new theory of eq 5.18 with a reaction range $r=7 \mathrm{~nm}$. The dashed lines indicate the asymptotic values of $\beta=2$ and $\beta=3$. Inset: predicted variation of effective reaction order for $r=0,1,2,5,10,20$ in units of lattice spacing.

The peak value of $\beta$ in Fig. 5-14 would correspond to a decay exponent of the population $\alpha \sim 0.46$. We note that this is very similar to the value reported by Russo et al. [71], Lüer et al. [13], and Zhu et al. [73]. The inset of Fig. 5-14 shows the predicted variation of $\beta$ for different value of reaction range $0,1,2,5,10,20$ in units of the lattice spacing $\Delta z$. Examination of equation 5.18 shows that a peak in $\beta$ is obtained when

$$
\left(1-\frac{\pi}{2} \frac{k_{r}}{k_{d}} r\right)<1
$$

i.e. when $r>\frac{2}{\pi} \frac{k_{d}}{k_{r}}$ or in terms of microscopic parameters $r>\frac{\Delta z}{p}$. The reaction range must 
exceed the particle spacing corresponding to crossover to the diffusion-limited regime, extending the reaction-limited range beyond that for short-range interactions.

\section{Determination of Diffusion time " $t_{0}$ ", Reaction time " $t_{1}$ ", and Cross over time " $t_{2}$ "}

We used the diffusion coefficient ' $D$ ' and the reaction rate constant ' $k_{r}{ }^{\prime}$ determined form Fig. 5-11 to calculate the diffusion time $t_{0}$, reaction time $t_{1}$, and cross over time $t_{2}$. The diffusion time is

$$
\begin{gathered}
t_{0}=\frac{L^{2}}{2 \pi D N_{0}^{2}} \\
t_{0}=\frac{(184)^{2}}{2 \pi(8.1) N_{0}^{2}}
\end{gathered}
$$

For $\mathrm{N}_{0}=145$ this time will $t_{0}=32 \mathrm{fs}$. This means that excitons encounter each other during the duration of pulse. This will still be true at the exciton number

$$
N_{0}>\sqrt{\frac{L^{2}}{2 \pi D t}}=\sqrt{\frac{665.2 p s}{t}}=\sqrt{\frac{665.2 p s}{0.1 p s}}=82
$$

At lower excitation power (for $\mathrm{N}_{0}<82$ ), excitons have not encountered each other during the duration of the pulse.

The value of diffusion time ( $t_{0}=32 \mathrm{fs}$ ) is much smaller that the reported dephasing time of $78 \mathrm{fs}$ measured with four wave mixing experiment (FWM) [101] and 120 fs for $(9,8)$ nanotubes measured using PL spectroscopy [103]. The dephasing time was reported to depend on the diameter of nanotubes [102] and was measured to be $98 \mathrm{fs}$ for nanotube with $0.9 \mathrm{~nm}$ diameter. These measurements were performed at lower excitation than in our experiments.

Exciton scattering time $\tau_{\text {scat }}$ depends on both exciton-phonon scattering and excitonexciton scattering. In our case, at high optical intensities, exciton-exciton scattering is dominant process contributing to scattering time because of large number of photogenerated excitons which are close to each other.

The reaction time is 


$$
\begin{aligned}
t_{1}=\frac{L}{k_{r} N_{0}} & =\frac{184}{(3.76) * 145} \\
t_{1} & =337 f s
\end{aligned}
$$

This means that even at the highest exciton density, which corresponds to the saturation condition, the excitons have not begun to interact during the duration of pulse. Therefore it seems unlikely that the cause of saturation is the high reaction rate.

As we defined $t_{2}$ as cross over time between reaction-limited and diffusion-limited regime, so in the case of high pump power, the value of $t_{2}$ will be

$$
t_{2}=\frac{2 \pi D}{k_{r}^{2}}=\frac{2 \pi(8.1)}{(3.76)^{2}}=3.6 p s
$$

And if we include the uncertainty in $k_{r}$ and $D$, then $t_{2}=(3.6 \pm 0.2) p s$ and the number of excitons was found from fit, where two power laws intersect

$$
N=\frac{k_{r} L}{2 k_{d}}=\frac{3.76 n m \cdot p s^{-1} \times 184 n m}{2 \pi \times 8.1 n m^{2} \cdot p s}=13.6
$$

The above calculation shows that diffusion-limited regime occurs for $1<N<14$ which corresponds to average exciton spacing greater than $13 \mathrm{~nm}$ and reaction-limited regime occurs for $N>14$ and for exciton spacing less than $13 \mathrm{~nm}$. The crossover between two regimes occurs at $t \approx 4 p s$.

\subsection{Conclusion}

In this chapter, we study the decay of photogenerated excitons in a solution of semiconducting carbon nanotubes in the regimes of many, few, and single exciton and found that multiple excitons decay through exciton-exciton annihilation. At short time and sufficiently high excitation, a fast intensity dependent decay is observed with positive deviation from $t^{-1 / 2}$ power law which can be explained by reaction-limited exciton-exciton annihilation. At long time, exciton decay follows approximately a $t^{-1 / 2}$ power law which is characteristic of diffusion-limited two body annihilation processes. We also determine the rate equation governing the exciton density $n$ directly from the experimental data and found 
that reaction-limited regime follows the classical rate equation for the bimolecular reaction i.e. $\frac{d n}{d t}=-k_{r} n^{2}$, whereas the diffusion-limited regime follows the anomalous rate equation for $1 \mathrm{D}$ diffusion i.e. $\frac{d n}{d t}=-k_{d} n^{3}$. The crossover between these different regimes occurs around $t=(3.6 \pm 0.2) p s$ which allows us to extract the ratio of reaction and diffusion coefficients directly.

The single exciton regime is observed at time $\sim 1 \mathrm{~ns}$, when excitons generated at the end of the nanotubes have sufficient time to diffuse to the centre and annihilate. This means that exciton-exciton annihilation ceases at time $>1$ ns and this single exciton has to decay through some other slow processes. From this information, we directly measured the maximum average number of excitons $N_{s}$ created per nanotube which is equal to $\sim 145$ at highest optical excitation. We observed the saturation of exciton population when separation become comparable to exciton spacing and found exciton size of $1.3 \mathrm{~nm}$ which is close to value reported previously [13]. Hence, we provided experimental evidence of the absorption saturation at high intensities which occurs due to Pauli exclusion between the fermionic constituents of the excitons.

We found that the analytical expression with short range interaction derived by Zhong and Ben-Avraham fitted very well with experimental data asymptotically but fails to fit in middle region, where the transition from reaction-limited to diffusion-limited is abrupt. Our simple model with long range (dipole-dipole) interaction fitted remarkably well with experimental data. From this fitting, we extract the diffusion coefficient of $(8.1 \pm 0.4) n m^{2} \cdot p s^{-1}$ and reaction rate constant of $k_{r}=(3.76 \pm 0.04) n m \cdot p s^{-1}$. We also found that diffusion-limited regime occurs for $1<N_{s}<14$ corresponding to average exciton spacing $>13 \mathrm{~nm}$ and reaction-limited regime for exciton spacing $<13 \mathrm{~nm}$. 


\section{CHAPTER 6}

\section{PUMP PROBE MEASUREMENTS OF COMOCAT SWNTS}

\subsection{Introduction}

This chapter presents an investigation of CoMoCat SWNTs in which the presence of disorder is found to affect the dynamics of exciton reactions. This chapter is organised in such a way that section 6.2 describes the experimental results obtained on two different CoMoCat SWNT samples. The comparison of CoMoCat results with those of the previously investigated HiPco SWNTs is presented in section 6.3. An interpretation of these results is given in section 6.4, where we provide evidence of diffusive and sub-diffusive transport of excitons on quasi 1D SWNTs and correlate the sub-diffusive transport to the presence of defects using previously measured Raman spectroscopy and X-ray photoelectron spectroscopy (XPS). Comparison of experimental results with Monte Carlo simulation of a 1D random walk of particles on a lattice with random distribution of barriers is also presented in the same section. A summary is given in the last section (section 6.5) of this chapter. 


\subsection{Pump Probe Measurements of CoMoCat SWNTs}

\subsubsection{Pump Probe Measurements of CoMoCat1 Sample $(7,6)$}

This section presents intensity dependant degenerate pump probe measurements of CoMoCat SWNTs (sample "CoMoCat1", discussed in chapter 4). The measurement wavelength of $1142 \mathrm{~nm}$ is in resonance with the first optical excited state $\left(E_{11}\right)$ of $(7,6),(8,4)$, $(9,2)$, and $(10,0)$ SWNT species as identified from their linear absorption as described in chapter 4. A variation of diffusion coefficient $(D)$ with laser power was observed in experiments on HiPco SWNTs (discussed in chapter 5). Hence, the measurements presented in this chapter were taken at a relatively low repetition rate of $10 \mathrm{kHz}$ in order to minimise heating while maintaining sufficient pump pulse amplitude to saturate the exciton density.

The pump-induced differential transmission $\left(\Delta \mathrm{T} / \mathrm{T}_{0}\right)$ is plotted in Fig. 6-1 for a pump pulse energy of $80 \mathrm{~nJ}$. The pump beam was focussed to a spot size of $\sim 102 \mu \mathrm{m}$ at the sample. The data is plotted on a double logarithmic scale up to $\sim 1 \mathrm{~ns}$.

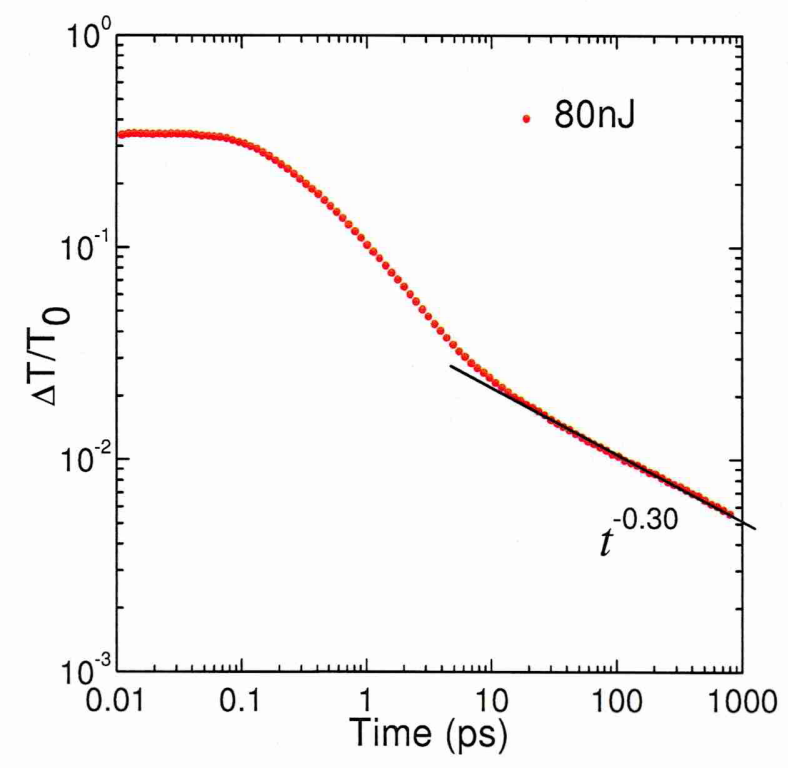

Figure 6-1: Pump probe data plotted on double logarithmic scale up to $\sim 1 \mathrm{~ns}$. The straight line indicates $t^{-0.30}$ power law included as guide to eye.

The optical transient shown in Fig. 6-1 is characterised by three distinct regimes:

1. At early times $(t<100 \mathrm{fs})$ there is little decay of the absorption saturation; 
2. At intermediate times $(100 f s \leq t \leq 4 p s)$, a power law decay with exponent $\alpha>1 / 2$ is observed; and

3. At long times ( $t \geq 10 \mathrm{ps}$ ), the differential transmission follows an asymptotic power law decay $t^{-|\alpha|}$ with exponent $\alpha \sim 0.30$.

The photobleaching transients for different pump fluences taken on CoMoCat 1 SWNTs at resonant excitation of first excited state $\left(E_{11}\right)$ are shown in Fig. 6-2. The pump fluence was varied from $35 \mu \mathrm{J} / \mathrm{cm}^{2}$ to $2.83 \mathrm{~mJ} / \mathrm{cm}^{2}$ for these measurements, yielding the pulse energies shown in Fig. 6-2. Pump and probe beams were focussed to spot size of $102 \mu \mathrm{m}$ and $64 \mu \mathrm{m}$ respectively at the sample.

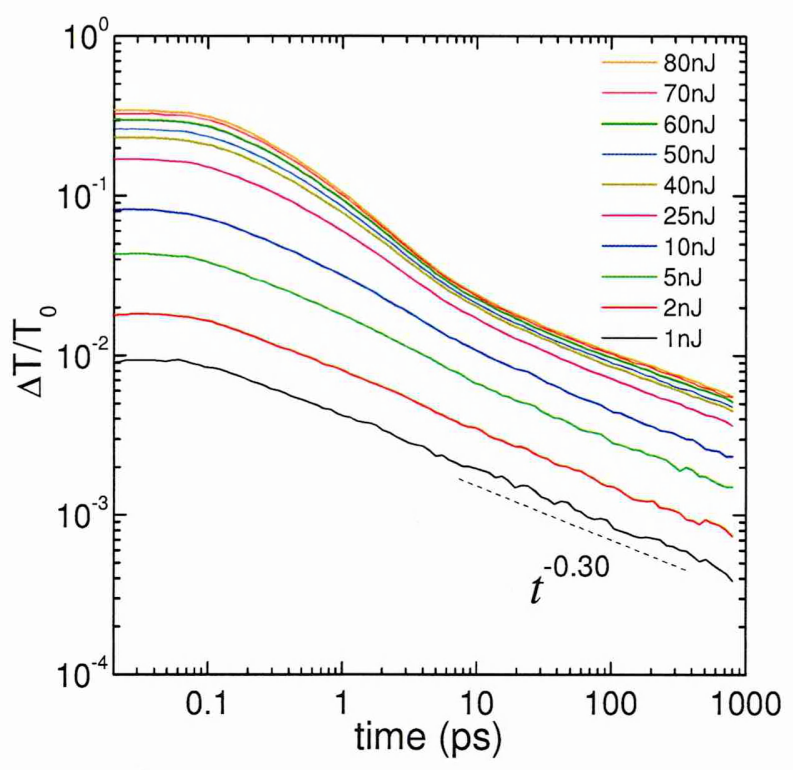

Figure 6-2: Pump probe data at different pulse energies up to $\sim 1$ ns, plotted on double logarithmic axis. The dotted line indicates $t^{-0.30}$ decay, included as a guide to the eye.

The data plotted in Fig. 6-2 shows that at intermediate times (100 fs $\leq t \leq 4 \mathrm{ps}$ ), an intensity-dependant fast decay with exponent $\alpha>1 / 2$ is observed for high pump pulse energy $\geq 20 \mathrm{~nJ}$ ( $200 \mu \mathrm{W}$ pump power); the magnitude of $\alpha$ increases with pump fluence. At long time ( $t \geq 10 \mathrm{ps}$ ), a decay exponent $\alpha<1 / 2$ was observed for all range of pump intensities used. This is different from the asymptotic $t^{-1 / 2}$ power law decay previously observed in the case of HiPco nanotubes (discussed in chapter 5).

Furthermore, we did not see a single-exciton regime, as observed in HiPco SWNTs where 
deviation from a $t^{-1 / 2}$ decay was found at times approaching $\sim 1 \mathrm{~ns}$. This may be due to the much longer nanotubes (average nanotube length $\sim 766 \mathrm{~nm}$ ) present in our CoMoCat sample as compared to HiPco (average nanotube length $~ 184 \mathrm{~nm}$ ). Using the value of diffusion coefficient measured previously in HiPco nanotubes, the time needed by single exciton to diffuse across the $766 \mathrm{~nm}$ tube is $\sim 11 \mathrm{~ns}$ which is beyond the range of measurement with our current setup.

As discussed previously in chapter 5, an effective time-dependent exponent $\alpha$ of power law can be determined through numerical differentiation of the experimental data. Hence we determined the logarithmic derivative of the signal $\left(S=\Delta T / T_{0}\right)$ and plotted it as function of time delay in Fig. 6-3. The data is apparently independent of pump energy for $t>10 \mathrm{ps,} \mathrm{and}$ the red line is an average of all the data in this region to show more clearly the asymptotic value of $\alpha$.

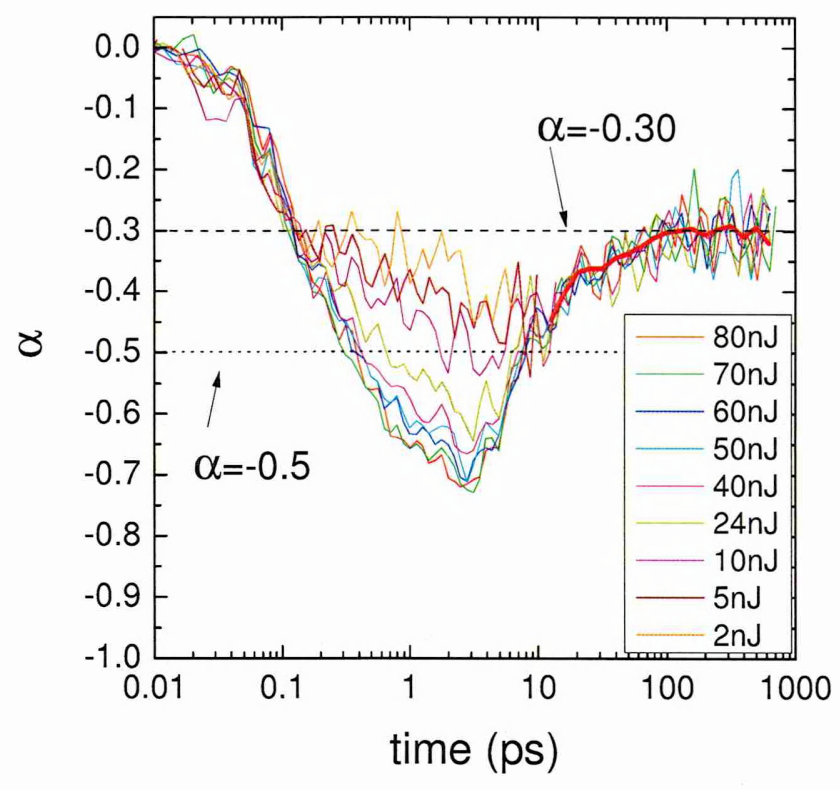

Figure 6-3: The logarithmic derivative of $S$ as function of pump probe delay. The dashed line corresponds to $\alpha=-0.30$ and dotted line corresponds to $=-0.5$. The red line is an average of all the data at different pump energies for $t>10 \mathrm{ps}$.

This figure reveals following

- At early times $(t<0.1 \mathrm{ps})$, the exponent is almost zero for all fluences because $d n / d t=0$ for time $t<t_{0}+t_{1}$ i.e. the time taken for the particles to meet and the first reaction to occur. 
- In the intermediate time (100 fs $<t<10 \mathrm{ps}$ ), the value of exponent increases with pump intensity and reaches a maximum value of $\alpha \sim 0.7$ at the highest intensity used in our measurements.

- At long times, all the traces saturate to $\alpha=(0.30 \pm 0.01)$ irrespective of excitation densities.

At intermediate times, the decay exponent varies approximately in the range -0.5 to -0.7 depending on the pump energy. According to equation 2.13 , it should approach exactly to $\alpha=-1$ at sufficiently high initial particle density; however this region was not reached in our experiment. The decay exponent $\alpha$ tends to zero at early times in order to satisfy the initial condition, given by the constant of integration of the classical (mean-field) rate equation 2.12. Hence we differentiated the data and plotted it against $\mathrm{S}=\Delta \mathrm{T} / \mathrm{T}_{0}$ on a double logarithmic axis in Fig. 6-4. Note that we have no scheme to calibrate the measurement signal $\mathrm{S}$ analogous to that used in chapter 5 , and so we plot dS/dt against $\mathrm{S}$ to find the functional dependence of decay rate on population.

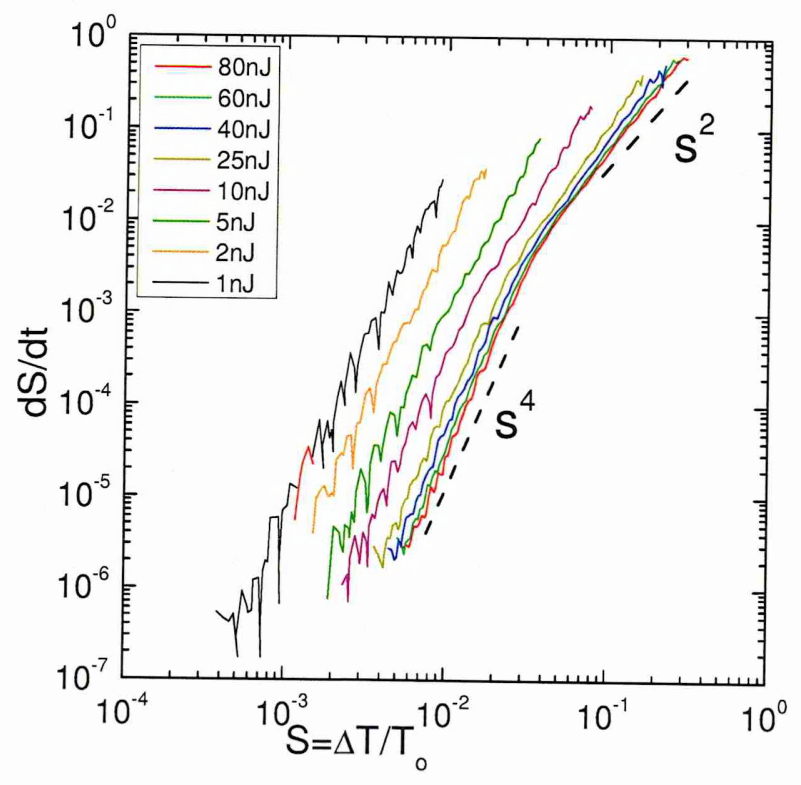

Figure 6-4: Time derivative of $S$ as function of $S$ plotted on double logarithmic scale. The dashed lines indicate regions of $S^{2}$ and $S^{4}$ and are included here for a guide to eye

It can be seen from Fig. 6-4 that the early dynamics under high excitation has approximately $S^{2}$ dependence similar to the HiPco nanotubes measured previously and can 
be similarly explained by reaction-limited exciton-exciton annihilation, occurring when the time for reaction to occur between two nearby particles is longer than the time it typical takes them to meet through diffusion.

Based on assumption that sample heating is negligible at this lower repetition rate, we rescaled the decay curves assuming that $D$ is constant and plotted them in Fig. 6-5 (a). The figure shows that all the traces are identical after $10 \mathrm{ps}$ and follow $t^{-|\alpha|}$ power law decay with decay exponent $\alpha=(0.30 \pm 0.01)$. We also differentiated the rescaled data and plotted it in Fig. 6-5 (b) for the highest six pump pulse energies which shows that the normalised curves for dS/dt are identical, so that the changes seen in Fig. 6-5 (a) at early times are purely due to changes in the initial condition (non-uniform illumination) and not in the underlying reaction rates.
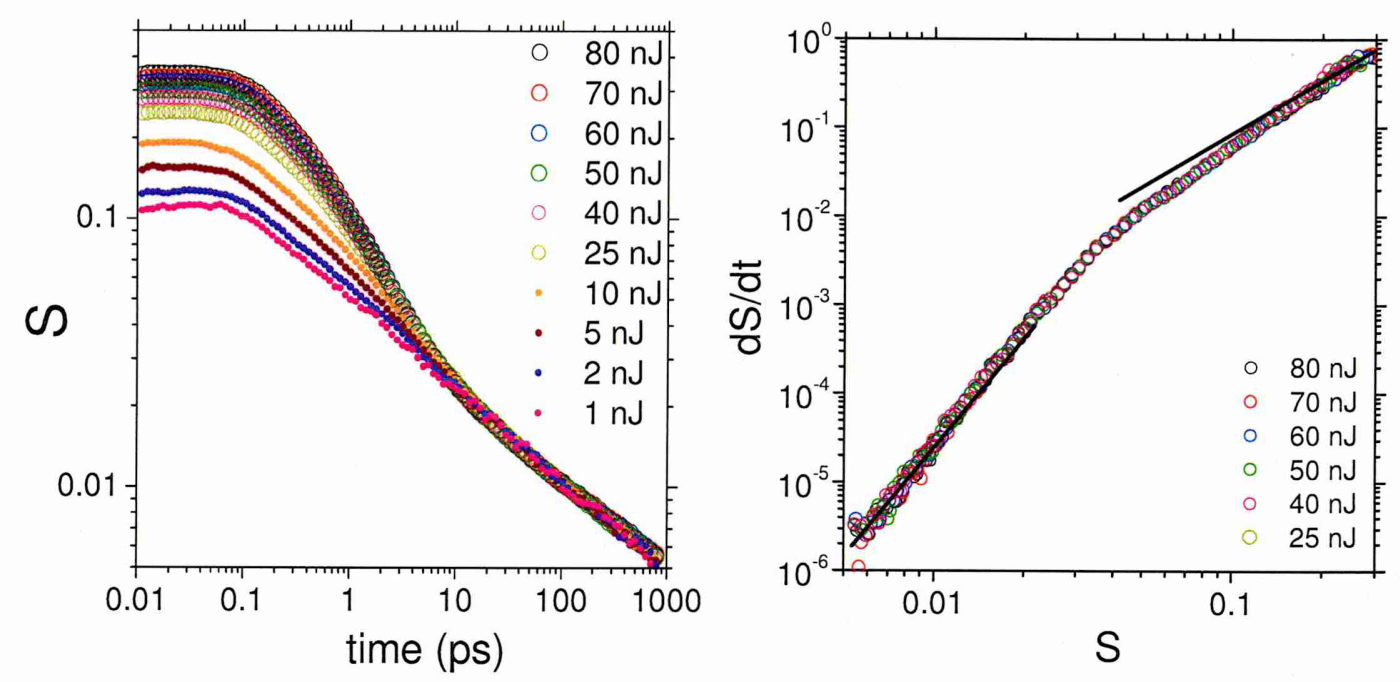

Figure 6-5: (a) Fluence dependent pump probe data of CoMoCat1 SWNTs taken at $1142 \mathrm{~nm}$, normalised at long time to show the universal behaviour. (b) Time derivative of $S$ as function of $S$ plotted on double logarithmic scale for highest 6 pulse energies, showing identical behaviour independent of initial excitation densities.

The data plotted in Fig. 6-2 suggests the saturation of exciton population at high pump energies. To see it more clearly, we plotted the maximum value of differential transmission $\left(\Delta \mathrm{T} / \mathrm{T}_{0}\right)$ against incident pump fluence in Fig. 6-6. It can be seen that the differential transmission begin to deviate from linear dependence on fluence at a fluence of $\sim 0.3 \times 10^{16}$ Photon $/ \mathrm{cm}^{2}$. This sublinear behaviour may be due to 'phase space filling effect' as recently reported by Lüer et al. [13] in SWNTs. A phenomenological way to 
characterize this saturation behaviour [133] is described by

$$
\frac{\Delta T}{T_{0}}=A *\left(1-e^{-\frac{f}{f_{s}}}\right) .
$$

where $f$ is fluence of pump pulse and $f_{s}$ is the saturation fluence. The solid line in the Figure 6-6 is a fit using this model with $A=0.45 \pm 0.01$ and a saturation pump fluence $f_{s}=(1.10 \pm 0.05) \times 10^{16}$ photons $/ \mathrm{cm}^{2}$. This saturation shows that there is limit on the maximum number of excitons which can be created on the nanotubes.

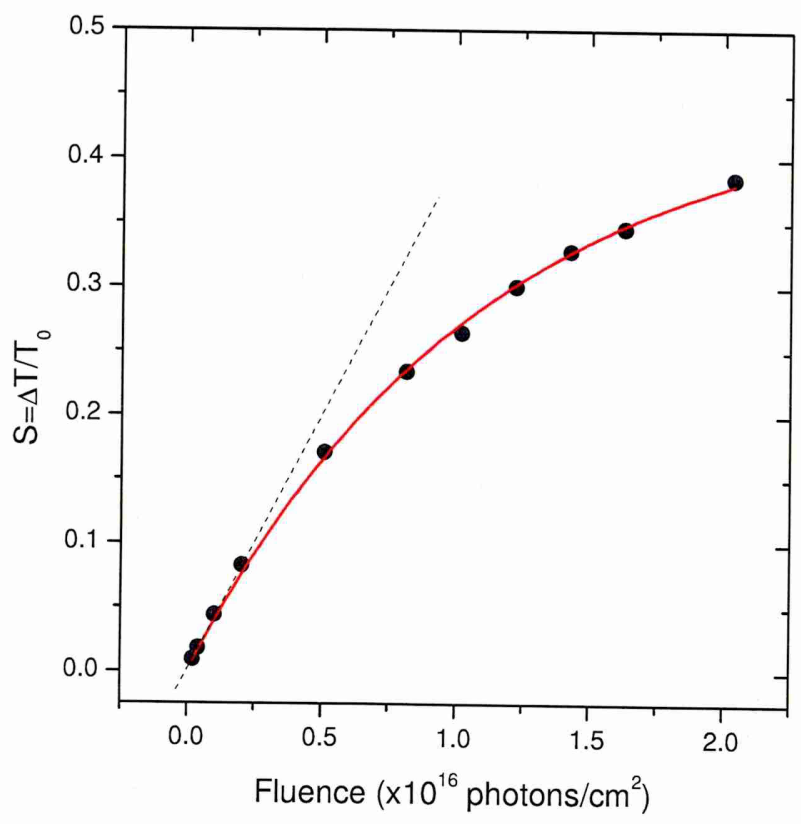

Figure 6-6: Pump fluence dependence of differential transmission at $t=0$ s fitted with the model described in [133]. The black dots are experimental data, red line is fit obtained with saturation model, and the dotted line is a linear dependence included here as a guide to the eye.

Most of the previous work reported saturation of the photoluminescence (PL) intensity at much lower pump fluence (2-5 orders of magnitude) e.g. Murakami et al. [50] observed PL saturation at fluence of $1 \times 10^{14}$ Photon $/ \mathrm{cm}^{2}$ in encapsulated SWNTs and Xiao et al. [39] recently reported PL saturation at even lower pump fluence of $\sim 6 \times 10^{11}$ Photon $/ \mathrm{cm}^{2}$ for air suspended SWNTs. These lower values of PL saturation are due to different underlying mechanism as compared to our case (e.g. see the section 2.5). 
The observed saturation in our CoMoCat SWNTs is also an order of magnitude higher than Lüer et al. [13] who reported saturation at pump fluence of $\sim 0.2 \times 10^{15}$ Photon $/ \mathrm{cm}^{2}$ for $(6,5)$ nanotubes. The higher value of fluence in our case than Lüer et al. [13] may be due to inhomogeneous excitation in our sample.

To investigate this further we compare the saturation at long and short times (each normalised to 1 for the highest pulse energy) as shown in Fig. 6-7. The signal at time zero $\mathrm{S}(t=0 \mathrm{ps})$ corresponds to the total absorption by the sample and increases linearly (dashed line) with pulse energy at low values, but saturates at high pump energy as described above. Assuming that the behaviour at long times is universal (independent of initial conditions) as was found for the HiPco SWNTs in chapter 5, then the signal at long times $S(t=100 \mathrm{ps})$ increases with pump energy because an increasing number of nanotubes are excited. This signal saturates when the exciton population on each of the nanotubes in the probed volume is saturated. The ratio of $S(0) / S(100)$ is proportional to the average exciton population per nanotube. This quantity increases very slowly with pump energy due to the combination of the inhomogeneous excitation and the saturation of exciton population.

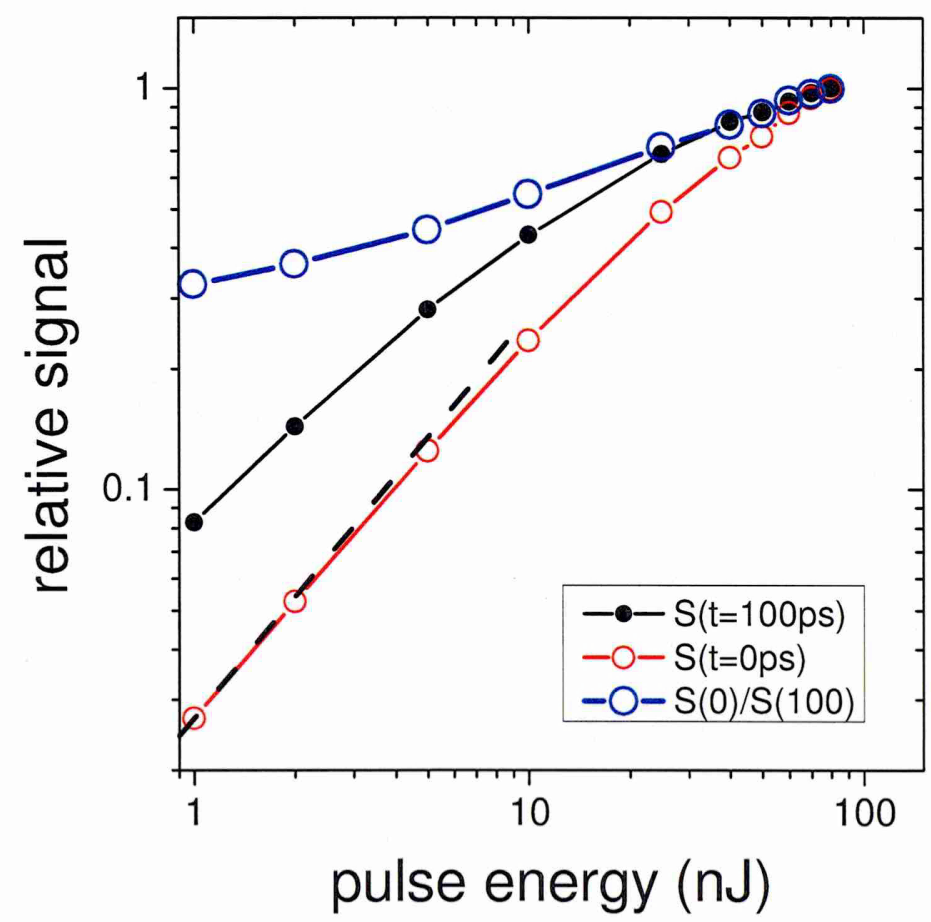

Figure 6-7: Normalise value of signal at $t=0 \mathrm{ps}$ (red line), $t=100 \mathrm{ps}$ (black line) and ratio of $S(0) / S(100)$ is plotted as function of pump pulse energy. The detail is given in the text. 


\subsubsection{Pump Probe Measurements of CoMoCat2 SWNTs}

In this section, we present pump probe measurements taken on a second CoMoCat SWNT sample ("CoMoCat 2") under similar conditions of single-colour resonant excitation of $E_{11}$ by pumping the sample at $1190 \mathrm{~nm}$ and at repetition rate of $100 \mathrm{kHz}$. The sample contains $57 \%$ of $(6,5)$ SWNT species and is prepared using density gradient ultracentrifugation. The detail is described in chapter 4 . The pump induced differential transmission $\left(\Delta \mathrm{T} / \mathrm{T}_{0}\right)$ for different pump fluences that span from $0.35 \mathrm{~mJ} / \mathrm{cm}^{2}$ to $3.19 \mathrm{~mJ} / \mathrm{cm}^{2}$ as function of delay $(t)$ is plotted in Fig. 6-8 (a). Because the repetition rate is high (a factor of 10 higher than for the results reported in the previous section), the diffusion constant is again increased at the high excitation powers.

The logarithmic derivative of data as function of time is plotted in Fig. 6-8 (b). As in the previous section, an intensity dependent fast decay $(|\alpha|>1 / 2)$ at intermediate times is observed. At long time ( $t \geq 10 \mathrm{ps}$ ), the exciton dynamics appears to follow the power law $t^{-|\alpha|}$ power law but with a slightly slower decay exponent $(\alpha=0.27 \pm 0.01)$ as compared to CoMoCat 1 SWNTs where $\alpha=0.30 \pm 0.01$. The values of decay exponent for the three curves in Fig. 6-8 are $\alpha=0.26 \pm 0.02, \alpha=0.27 \pm 0.02$, and $\alpha=0.28 \pm 0.02$; i.e. the laser-induced heating does not significantly affect the exponent of the decay in the diffusion-limited region.
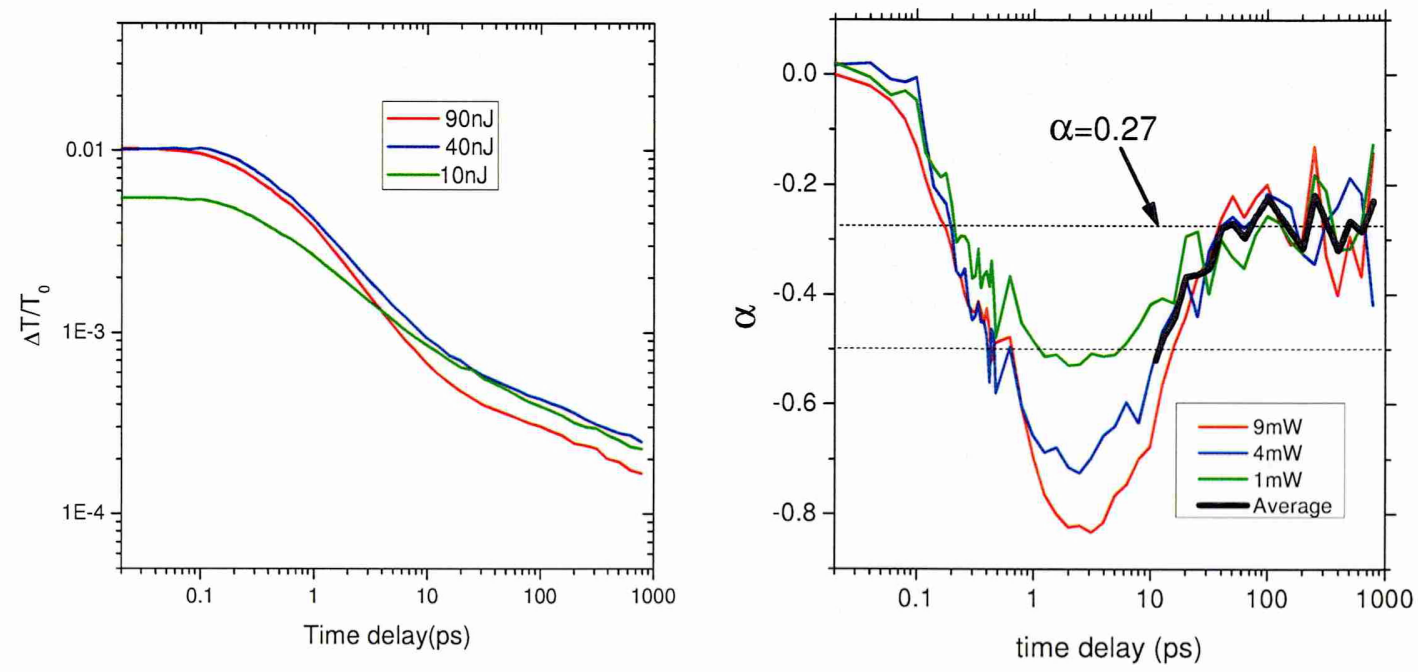

Figure 6-8: (a) Pump probe traces of CoMoCat2 taken at different pump fluences (b) decay exponent of power law obtained from the derivative of logarithmic data. The heavy line is an average of the three curves for $t>10$ s. The dotted lines are guides to the eye. 


\subsection{Comparison of CoMoCat SWNTs with ss-DNA HiPco SWNTS}

In this section, we compared the degenerate pump probe optical transients taken on three different samples (CoMoCat1, CoMoCat2, and previous studied ss-DNA wrapped HiPco SWNTs). This comparison is important to distinguish different kind of exciton transport in two different quasi 1D carbon nanotubes systems (CoMoCat and HiPco SWNTs). The data taken on CoMoCat1, CoMoCat2, and HiPco SWNTs at the highest pump fluence of $2.83 \mathrm{~mJ} / \mathrm{cm}^{2}, 3.19 \mathrm{~mJ} / \mathrm{cm}^{2}$, and $3.68 \mathrm{~mJ} / \mathrm{cm}^{2}$ respectively is shown in Fig. 6-9, normalised to the signal at zero time. The curves are shifted vertically with respect to each other for comparison. The behaviour at early and intermediate times is very similar, with the main difference between the three curves being the exponent of the long-time asymptote.

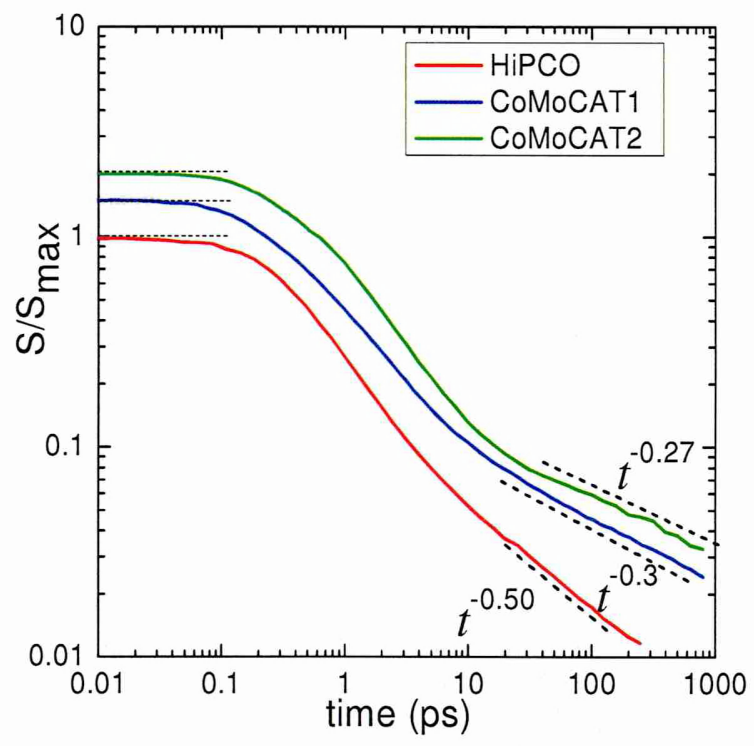

Figure 6-9: Normalised pump probe data taken on ss-DNA wrapped HiPco and CoMoCat SWNTs at highest pump fluence. $S_{\text {max }}$ is maximum value of signal $S=\Delta T / T_{0}$ measured at $t=0$. The dotted lines are included as a guide to the eye. The curves are shifted vertically with respect to each other for the comparison.

The decay exponents are compared in Fig. 6-10 (a). At times up to $10 \mathrm{ps,} \mathrm{the} \mathrm{three} \mathrm{samples}$ show similar behaviour, but their long-time asymptotic decay is clearly different. In the case of CoMoCat1 nanotubes, the decay exponent is $\alpha=(0.30 \pm 0.01)$; for CoMoCat2 nanotubes, it is $\alpha=(0.27 \pm 0.01)$ and for ss-DNA wrapped HiPco nanotubes, it is 
$\alpha=(0.46 \pm 0.01)$

The derivative dS/dt (proportional to the reaction rate) is shown in Fig. 6-10 (b). At large $\mathrm{S}, \mathrm{dS} / \mathrm{dt}$ varies as $\mathrm{S}^{2}$ in all samples. This shows the existence of a reaction-limited regime at high exciton population, which is independent of the factors which cause the different behaviours at long times.
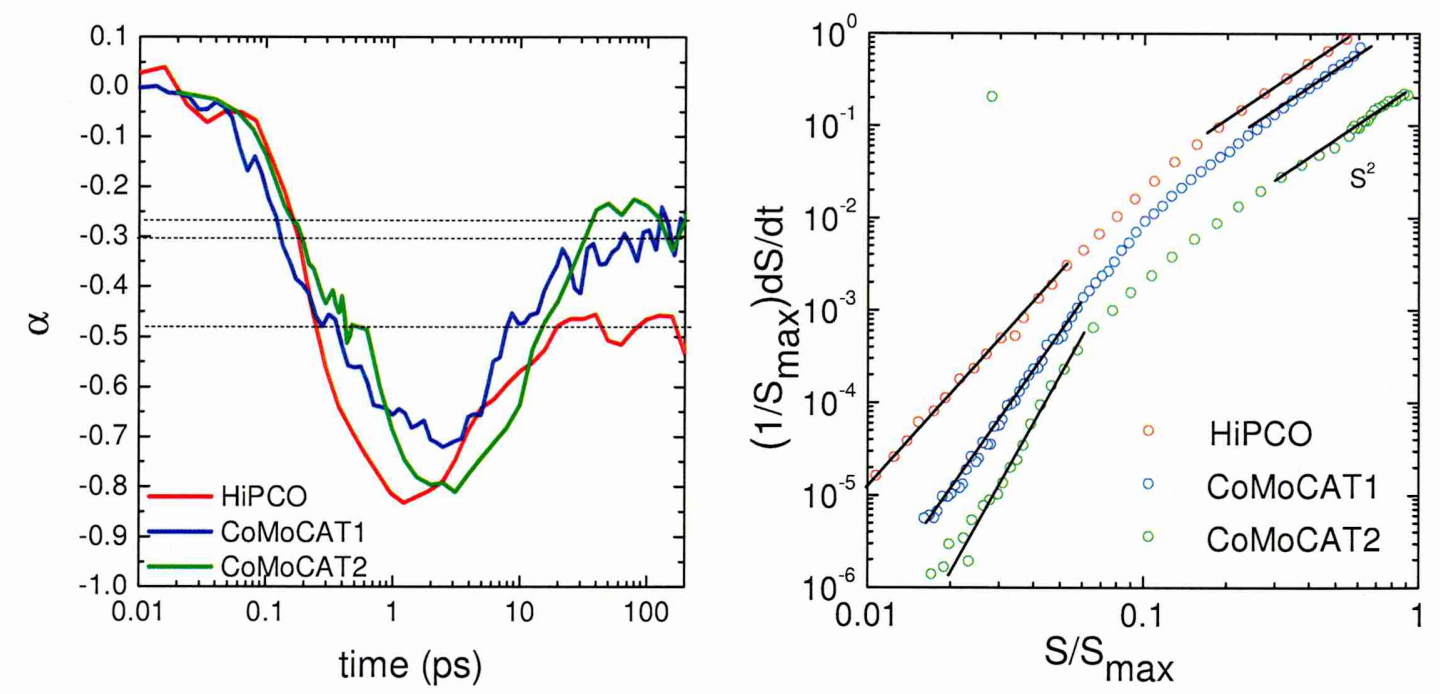

Figure 6-10: (a) Logarithmic derivative which is equal to exponent $\alpha$ as function of time for three different nanotubes samples. The dashed lines are drawn as a guide to the eye. (b) Linear derivative of $S$ as a function of $S$. A line with gradient $n$ corresponds to rate equation $d S / d t=S^{n}$, showing three distinct dependence at low exciton density. Curves are normalised for comparison.

\subsection{Interpretation of Pump Probe Results}

In this part of the chapter, a possible interpretation of the pump probe results presented above will be discussed. In first section 6.4.1, the power law with slow decay at long time will be discussed in terms of diffusive and sub-diffusive transport of excitons. In the same section, we will correlate this long time behaviour to the defect density in nanotubes using previously measured Raman spectroscopy and X-ray Photoelectron spectroscopy (XPS) results. In second section 6.4.2, exciton population will be estimated using absorption cross section approach. The asymptotic rate equation governing three different regimes will be discussed in third section 6.4.3, where we will deduce reaction rate coefficients from 
experimental data directly. In section 6.4.4, interpretation of results in terms of anomalous reaction and diffusion will be given, where we will show that our modified long range subdiffusive model best describes the experimental data. In section 6.4.5, a comparison of experimental results with Monte Carlo simulations will be discussed and in the last section of the chapter, a summary will be given.

\subsubsection{Interpretation of Asymptotic Long Time Slow Decay and Subdiffusive Transport}

The traces in Fig. 6-2 and Fig. 6-3 show that in limit of long times, the exciton population in the CoMoCat SWNTs follows a power law $t^{-|\alpha|}$ with decay exponent $\alpha=0.30 \pm 0.01$. This slow asymptotic decay in CoMoCat SWNTs is strongly suggestive of sub-diffusive transport of particles (excitons) in a disordered one-dimensional system as discussed in section 2.4.4.2. The disorder can be strong which results in behaviour best described by a fractal dimension or weak due to the presence of barriers, wells and traps, and described by anomalous diffusion. The disorder in carbon nanotubes is one example of weak disorder in a one-dimensional system which arises due to the presence of the defects.

Hence the observed sub-diffusive transport of excitons in CoMoCat SWNTs with slow exciton decay $(|\alpha|<1 / 2)$ may be due to the presence of higher defect concentration than in HiPco nanotubes. In order to verify this hypothesis, the defects in both nanotube samples were measured using Raman spectroscopy as discussed in section 4.5.3 in which the $I_{D} / I_{G}$ ratio of CoMoCat and HiPco SWNTs was compared. It was found that this ratio is two times higher in the CoMoCat nanotubes as compared to HiPco nanotubes, which indicates a higher defect density. From the value of $I_{D} / I_{G}$ measured in the CoMoCat sample $\left(I_{D} / I_{G} \sim 0.21\right)$, the average distance between defects can be estimated as $s \sim 22 \mathrm{~nm}$ using a recently reported quantifying approach of defects in graphene [116].

We also verified our approach using XPS as discussed in section 4.6 in which the width of the $\mathrm{sp}^{3}$ peak (associated with defects) as well as the ratio of area of $\mathrm{sp}^{3}$ to $\mathrm{sp}^{2}$ graphitic carbon peaks was compared. It was found that the width of $\operatorname{sp}^{3}(\mathrm{FWHM} \sim 2.82 \mathrm{eV})$ and ratio of area of $\mathrm{sp}^{3}$ to $\mathrm{sp}^{2}\left(\frac{A_{s p^{3}}}{A_{s p^{2}}} \approx 1.44\right)$ in CoMoCat is higher than HiPco in which the width of $\mathrm{sp}^{3}$ is $2.76 \mathrm{eV}$ and the ratio $\frac{A_{s p^{3}}}{A_{s p^{2}}}$ is $\sim 1.00$. These findings supported our conclusion that CoMoCat 
SWNTs have more defects as compared to DNA-wrapped HiPco SWNTs.

\subsubsection{Estimation of Exciton Population}

Before we can determine reaction rate coefficients for the different regimes identified above, we must determine the exciton density $n$ corresponding to a measured change in differential optical transmission. In the case of the HiPco SWNTs discussed in chapter 5, the proportionality constant between transmission change and exciton density was obtained by measuring the transmission change at a density corresponding to a single exciton per nanotube. However, the CoMoCat SWNTs were longer and hence the single exciton regime could not be observed. We therefore determined the exciton density from the measured transmission change by estimating the absorption cross-section following the approach of Islam et al. [72]. The formula for determining the saturated exciton density in 1D is [71, 133]

$$
n_{s}=\frac{\sigma_{S w N T}}{L} *\left\{\frac{F_{e}}{\hbar \omega}\right\}
$$

where $L$ is average length of nanotubes (measured with an AFM to be $(\sim 766 \pm 48) \mathrm{nm}$ ), $\omega$ is the frequency of excitation pulse, $F_{e}$ is the excitation fluence and the quantity in the bracket is the number of photons per pulse impinging on an area of $1 \mathrm{~cm}^{2} . \sigma_{S W N T}$ is the optical absorption cross section of carbon nanotubes and is given by [71]

$$
\sigma_{S w N T}=\frac{\sigma_{m o l}}{N_{A}} * \frac{8 \pi R_{n} L}{3 \sqrt{3} a_{0}^{2}}
$$

where $R_{n}$ is the radius of nanotube, $a_{0}$ is the graphene lattice constant, $N_{A}$ is Avogadro's number, and $\sigma_{m o l}$ is the absorption cross section of carbon and has been quantified as approximately $2 \times 10^{5} \mathrm{~cm}^{2} / \mathrm{mol}$ [72].

Using equations 6.2 and 6.3 , the saturated exciton density calculated is $\sim(7.2 \pm 0.6) \times 10^{6} \mathrm{~cm}^{-1}$ which corresponds to a minimum exciton spacing of $(1.4 \pm 0.1) \mathrm{nm}$. This exciton spacing is consistent with the value obtained for HiPco SWNTs in chapter 5, where the similarity to theoretical calculations of the exciton size was noted and interpreted as evidence for Pauli exclusion of the constituent Fermions making up the exciton. 
Using this value of exciton size, we determined the maximum average number of excitons per nanotube which is $\sim 560$ excitons. This allows us to extract the proportionality constant between the maximum transmission change and maximum exciton density.

\subsubsection{Determination of Reaction Rate Coefficients}

The reaction rate $\mathrm{dN} / \mathrm{dt}$, where the exciton number $\mathrm{N}$ is determined from the calculation given above, is plotted as a function of $\mathrm{N}$ in Fig. 6-11. The data shown is for sample CoMoCat 1 at a higher pump pulse energy of $100 \mathrm{~nJ}$ (this was chosen because it most clearly exhibits the three distinct regions described here). At high and low populations, the reaction rate, $-d n / d t$ where $n=N / L$, is described by a reaction-limited term $k_{r} n^{2}$ and an anomalous diffusion-limited term $k n^{(1+2 / \gamma)}$ respectively, where $k=\left(\pi D_{\gamma}\right)^{1 / \gamma}$ and $D_{\gamma}$ is the anomalous diffusion coefficient defined in chapter 2 (e.g. see equation 2.11). It is notable that the transition between these two regions is more gradual than the transition between the asymptotic regions for HiPco SWNTs.

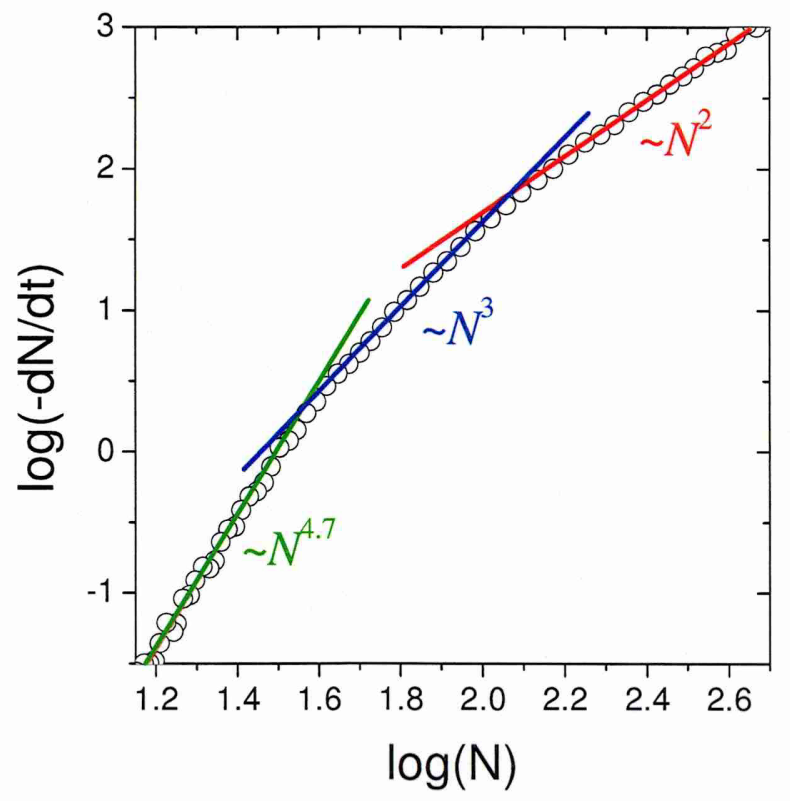

Figure 6-11: Time derivative of data for sample CoMocat1 with $100 \mathrm{~nJ}$ pump energy. The three different regimes are clearly seen in the figure. Straight lines corresponding to these different regimes are included as a guide to the eye.

An inspection of Fig. 6-11 suggests the existence of an intermediate region with a reaction 
rate of $\pi D n^{3}$ limited by normal diffusion. A fit to the data in each of these regions gives values of $\quad k_{r}=(3.7 \pm 0.3) \mathrm{nmps}^{-1}, \quad D=(8 \pm 1) \mathrm{nm}^{2} \mathrm{ps}^{-1}, \quad$ an $D_{\gamma}=(33 \pm 11) \mathrm{nm}^{2} \mathrm{ps}^{-0.54}$. The values of $k_{r}$ and $D$ are remarkably close to the values obtained for the same species of HiPco SWNTs in chapter 5, indicating that these are intrinsic properties independent of the method of synthesis, and not strongly dependent on the presence of surfactants or DNA. The significant difference between the samples lies in the anomalous diffusion term which is related to the density of defects.

\subsubsection{Simple Theoretical Model for Reaction Kinetics with Anomalous Reaction and Diffusion}

In order to further justify our identification of the three different regimes in Fig. 6-11, we modify our previous model of diffusion-limited reactions (described in section 5.3.2) to include this sub-diffusive behaviour. The schematic of model for defective CoMoCat nanotube is shown in Fig. 6-12, in which $x$ is the separation between excitons and $r$ is the interaction range defined previously. In addition to these parameters, we define another parameter $s$ as the separation between defects.

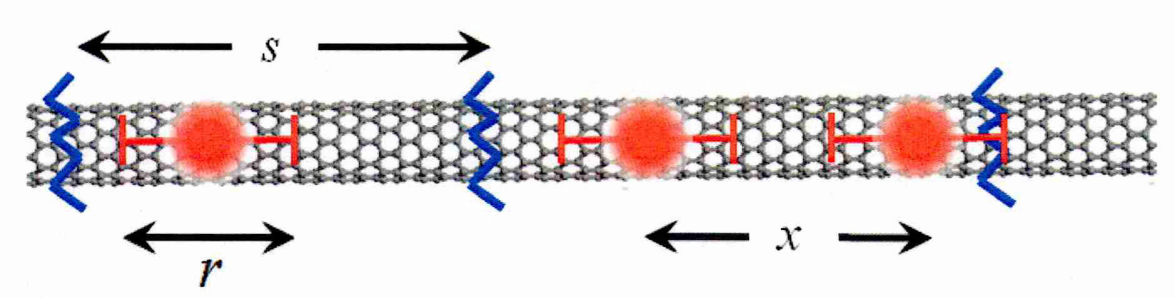

Figure 6-12: Schematic of defective nanotube; $r$ is the interaction range, $x$ is the average distance between excitons, and $s$ is the separation between defects.

In our sub-diffusive long range model, we define the three different regimes depicted in Fig. 6-13:

1. A reaction-limited regime, when separation between excitons is less that the interaction range i.e. $x<r$. In this regime, the decay of exciton population is limited by the intrinsic annihilation rate and can be explained using the mean-field classical rate equation given in equation 2.12 .

2. A normal diffusion-limited regime, when separation between the excitons is greater 
that the interaction range but significantly less than the separation between defects i.e. $r<x \ll s$. The transport of excitons in this regime can be explained by normal diffusion according to equation 2.15 .

3. A subdiffusion-limited region when separation between excitons is more that the separation between defects i.e. $x \gg s$. In this region, the transport of excitons is slowed down due to presence of defects and becomes sub-diffusive.

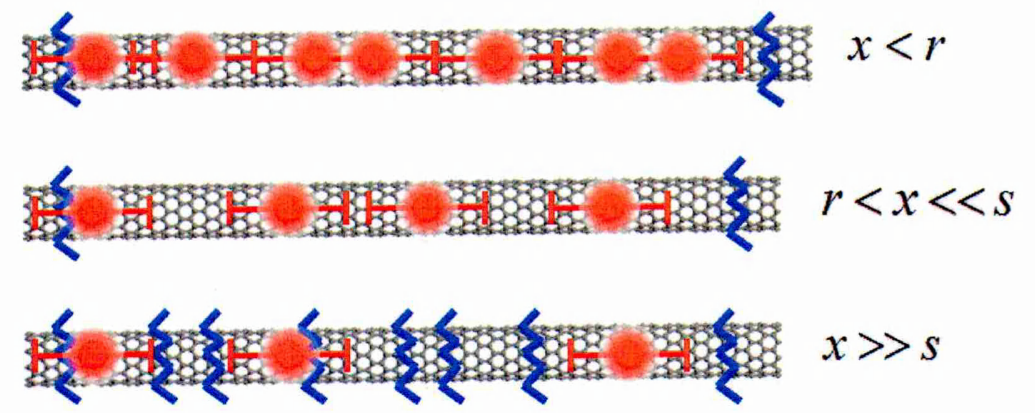

mean-field

normal diffusion

anomalous reaction

anomalous diffusion

anomalous reaction

Figure 6-13: Schematic of nanotubes with defects showing three different cases: (1) reaction-limited process when separation between excitons is less than their interaction range, (2) normal diffusion when the their separation is greater than interaction range but less that the defect separation, and (3) subdiffusion when the separation between excitons is greater than the defect separation.

The first two regions (reaction-limited and diffusion-limited) were already seen in HiPco SWNTs (discussed in detail in section 5.3.2). The sub-diffusive transport of excitons in SWNTs due to defects is new and has not been observed previously. The rate equation for sub-diffusive transport of exciton on nanotubes can be written easily using equation 2.11 .

$$
-\frac{d t}{d n}=\frac{1}{n}\left(\frac{\overline{x^{2}}}{4 D_{\gamma}}\right)^{1 / \gamma}
$$

With the introduction of a long-range interaction, equation 6.4 becomes

$$
-\frac{d t}{d n}=\frac{1}{n}\left(\overline{\frac{(x-r)^{2}}{4 D_{\gamma}}}\right)^{1 / \gamma}
$$


This rate equation is combined with equation 5.16 to describe the experimental data of CoMoCat SWNTs completely. Thus

$$
\begin{array}{cc}
-\frac{d t}{d n}=\frac{1}{k_{r} n^{2}} & (x<r) \\
-\frac{d t}{d n}=\frac{1}{k_{r} n^{2}}+\frac{1}{n} \overline{\frac{(x-r)^{2}}{4 D}} & (x>r, x<s) \\
-\frac{d t}{d n}=\frac{1}{k_{r} n^{2}}+\frac{1}{n}\left(\overline{\frac{(x-r)^{2}}{4 D_{\gamma}}}\right)^{1 / \gamma} & (x>s>r)
\end{array}
$$

Equation 6.6a is valid for reaction-limited regime where separation between neighbouring excitons is less than reaction range. This is the regime where the exciton population is so high that negligible transport is required before annihilation. The diffusion-limited regime occurs when the separation between excitons is large compared to their interaction range and follows equation $6.6 \mathrm{~b}$ and equation $6.6 \mathrm{c}$ depending on the exciton separation as compared to defect separation. If exciton separation is less than separation between defects, then the dynamics follow normal diffusion equation $6.6 \mathrm{~b}$ but if exciton separation is large compared to defects separation, then decay follows sub-diffusive equation 6.6c.

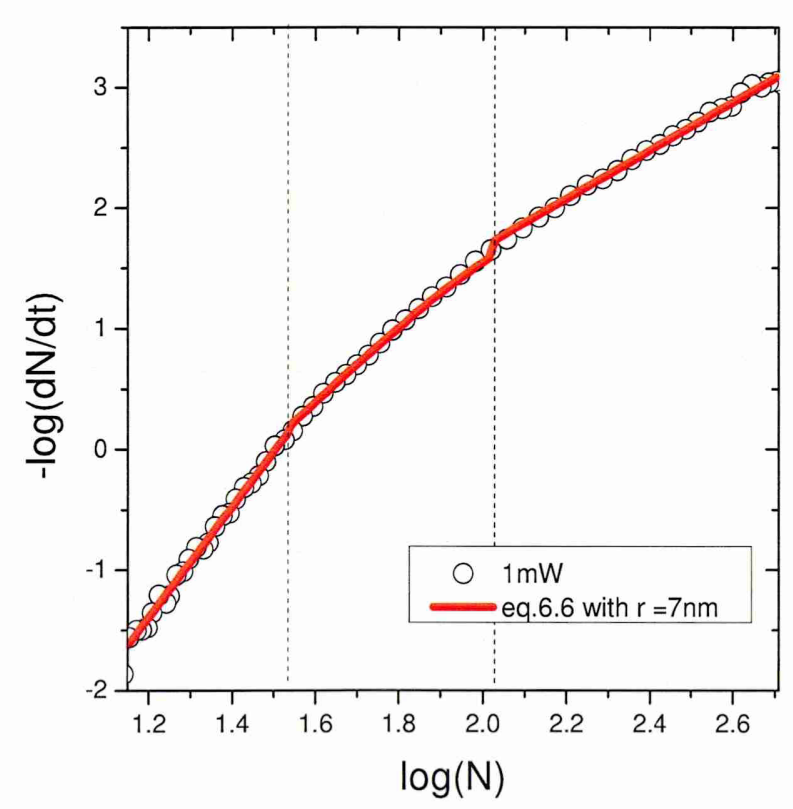

Figure 6-14: Experimental data fitted piecewise with equation 6.6. Symbols are experimental data; red line is fit to data using eq.6.6. The vertical dotted lines show the three different regions used for piecewise fitting. 
We perform a piecewise fit (using common parameters) to the three different regions described by equation 6.6 (shown in Fig. 6-14) using length of nanotube $L=(766 \pm 48) \mathrm{nm}$ measured with AFM, $s=(22 \pm 2) n m$, determined quantitatively from Raman spectroscopy, and $\gamma / 2=0.27 \pm 0.1$, determined from pump probe data. In order to make sure that asymptotic behaviour is reproduced, a reaction rate of $k_{r}=(3.7 \pm 0.3) n m . p s^{-1}$, subdiffusion coefficient of $\left(D_{\gamma}=(33 \pm 11) n m^{2} \cdot p s^{-0.54}\right.$, and diffusion coefficient of $D=(8 \pm 1) n m^{2} \cdot \mathrm{ps}^{-1}$ were used for the fitting. From the fitting, we obtained a interaction length of $r=(7.30 \pm 0.01) \mathrm{nm}$. The value of $r$ obtained is very similar to the value obtained for the HiPco SWNTs.

As a further check, the experimental data was also fitted using equation 6.6 with the same parameters but (i) with short range interaction $r=1.4 \mathrm{~nm}$, equal to exciton size (blue curve), (ii) without normal diffusion (red curve), and (iii) without subdiffusion (green curve) in Fig. 6-15. This figure shows that for a complete description of the experimental data, all three decay behaviours along with long range interaction are important. This analysis shows the importance of describing the spatio-temporal aspects of the reaction kinetics. A rate-equation approach [53] cannot describe the different regimes which depend on the relative sizes of the average particle separation, interaction range, and defect separation.

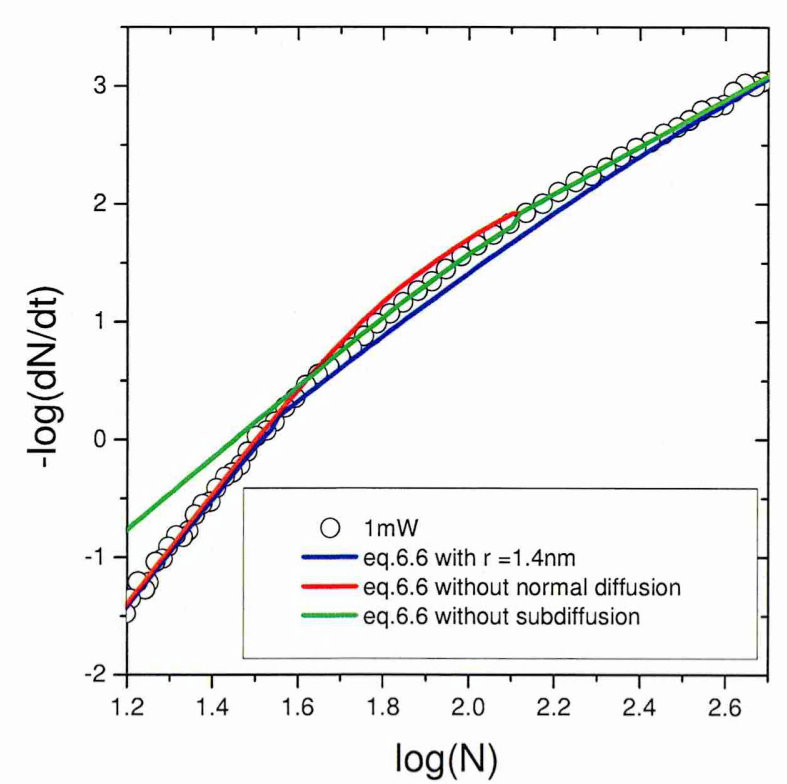

Figure 6-15: Experimental data fitted with equation 6.6 with the same parameters used in Fig. 6-14. Symbols are experimental data, blue line is a fit with short range interactions, green line is fit without subdiffusion, and red line is fit achieved without normal diffusion. 


\subsubsection{Comparison with Monte Carlo Simulation}

In order to understand the effect of defects on the diffusive transport of excitons in 1D system, a Monte Carlo simulation of random walk of particles on 1D lattice in the presence of potential barriers was performed. These simulations were carried out by A. Moretti within an undergraduate project supervised by Prof J. Allam, and are described here to justify our earlier interpretation of the dynamical results based on analytical approximations. Modification was made to previous Monte Carlo simulations of diffusion-limited reactions [134] to include sub-diffusive behaviour caused by the presence of potential barriers. Note that long-range interactions were not included in the simulations described in this section.

The potential barriers were randomly distributed over a simple 1D lattice and barrier heights are also chosen to be random. Transport of particles across these barriers is thermally activated, and particles which encounter each other are assumed to annihilate. Figure 6-16 shows how the barriers affect the annihilation probability. The dwell time (time spent on a particular lattice site averaged over the entire simulation) is enhanced at the low energy side of a potential barrier due to reflections, and depleted on the high energy side due to particles which diffuse across the boundary. The decay length of such enhancements and depletions is about $3 \mathrm{~nm}$. The reaction probability is found to be similarly affected but with stronger enhancements due to the dependence on the square of the local concentration.
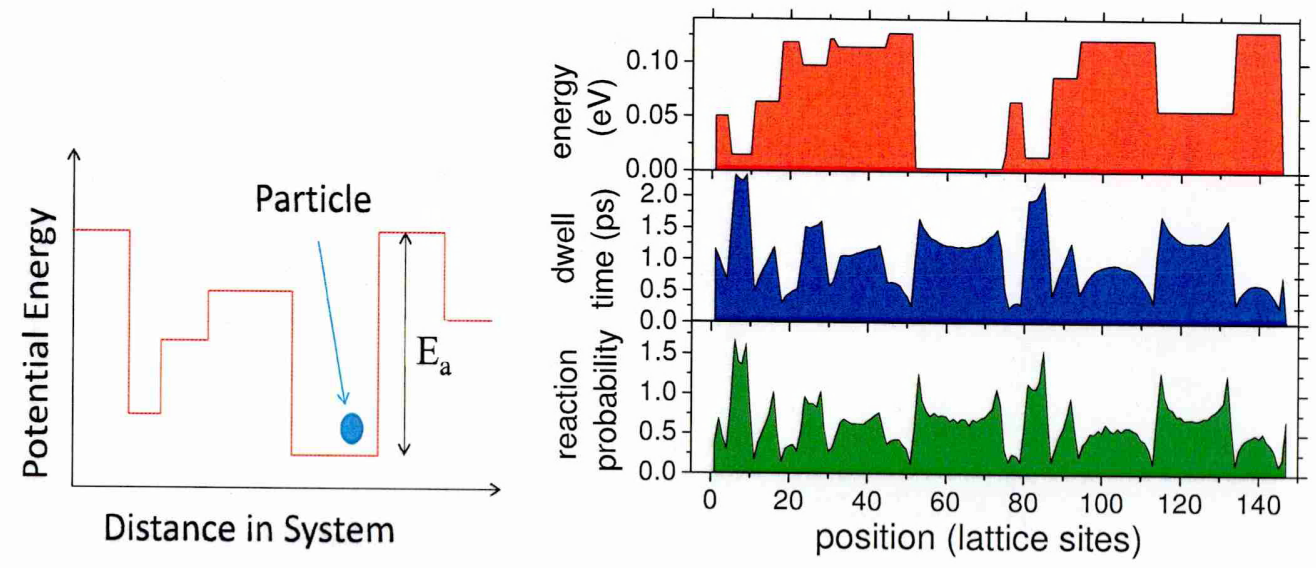

Figure 6-16: Monte Carlo simulation of random walk on $1 D$ lattice (figure provided by A. Moretti and J. Allam)

The results of simulations for a barrier height of $50 \mathrm{meV}$ are plotted in Fig. 6-17. In Figure 
6-17 (a), the population of excitons is plotted as function of time. In Fig. 6-17 (b), decay exponent $\alpha$ is plotted as function of time. Both Figures show that the presence of defects has a negligible effect on decay of excitons at early time. This is due to the fact that reactive particles are very close to each other and negligible transport is required to meet each other and annihilate, i.e. the reaction-limited regime. A difference in exciton dynamics is clearly seen at long times, where the decay of exciton population follows normal diffusion in the absence of potential barrier and anomalous diffusion (subdiffusion) in the presence of sufficient defect density (e.g. see Fig. 6-17 (a)). These simulation results are similar to the pump probe experimental results obtained on different SWNTs samples (e.g. see Fig. 6-9 and Fig. 6-10 (a)) which confirmed that as defect density increases the exciton transport changes from diffusive to sub-diffusive in a $1 \mathrm{D}$ system and leads to a reduction in the decay rate.
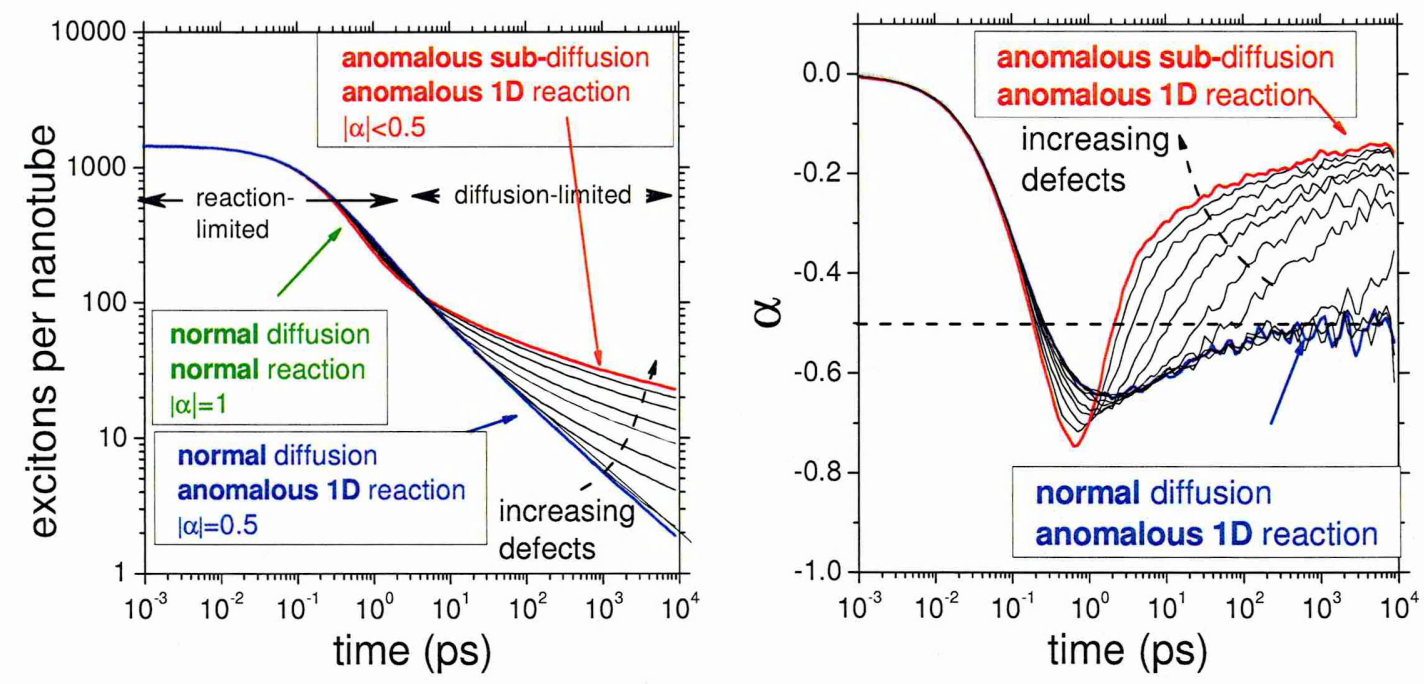

Figure 6-17: Results of Monte Carlo simulation performed on 1D lattice. (a) Exciton population is plotted as function of time. (b) Decay exponent " $\alpha$ " of power law which shows that reduction of the decay exponent is observed at sufficient defect density. (Figure provided by A. Moretti and J. Allam).

To investigate the role of the diffusive transport on the reaction kinetics, the mean square displacement of diffusing particles is plotted as a function of time on double logarithmic axis in Fig. 6-18 (a). As the defect concentration increases, the slope of curve decreases at long time and nonlinearity between the mean square displacement and time increases. This leads to a decrease in the diffusion coefficient. At early time, all curves show the normal diffusion due the fact that particles are very close to each other and did not encounter the defects yet. 
In Fig. 6-18 (b), the asymptotic value of the decay exponent $\alpha$ at long time is plotted against defect spacing which shows that the decay exponent $\alpha$ decreases with the logarithm of the defect concentration. For the parameters of this simulation (barrier height $=50 \mathrm{meV}$, diffusion coefficient $=15.5 \mathrm{~nm}^{2} \mathrm{ps}^{-1}$, short-range reactions with probability $=0.24$, temperature $294 \mathrm{~K}$ ), a defect separation of about $25 \mathrm{~nm}$ corresponds to the experimentally observed value of $\alpha \sim 0.27$.
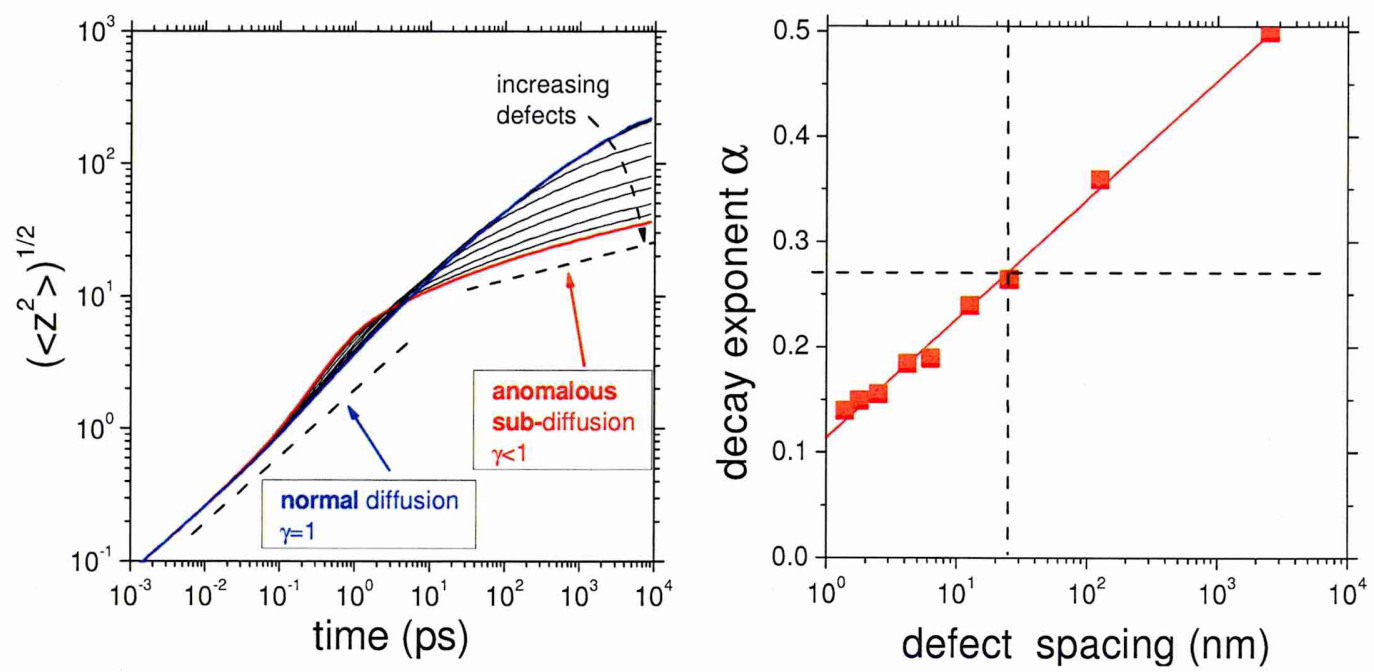

Figure 6-18: (a) The mean square displacement of the diffusing particle as a function of time which exhibits sublinear behaviour at sufficient defect density. (b) The decay exponent is plotted as a function of defect spacing (figure provided by A. Moretti and J. Allam).

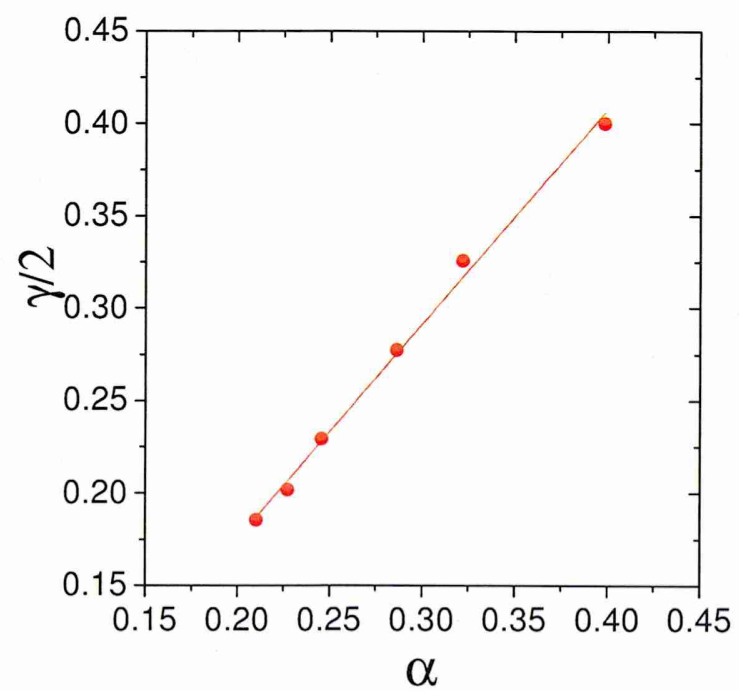

Figure 6-19: Diffusion exponent $(\gamma / 2)$ plotted against reaction exponent $(\alpha)$, red line is linear fit with slope $\sim 1$. 
In order to investigate the fundamental relations of anomalous diffusion-limited reaction rates, I calculated the reaction exponent $\alpha$ at 100 ps from Fig. 6-17 (a) and the diffusion exponent $\gamma / 2$ from Fig. 6-18 (a) and plotted them in Fig. 6-19, showing a linear relationship. A slope of $1.16 \pm 0.04$ is obtained from a linear fit.

\subsection{Conclusion}

In conclusion we have provided experimental evidence for diffusive and sub-diffusive transport of excitons in quasi 1D SWNTs. This evidence is achieved through pump probe measurements at resonant excitation of $E_{11}$ transition of SWNTs. We found that exciton decay follows a $t^{-|\alpha|}$ power law over four decades in time. This power law dependence on time in SWNTs is strongly suggestive of diffusion-limited reaction. At early time and high intensity, the pump probe traces shows positive deviation from $t^{-1 / 2}$ power law due to reaction-limited exciton-exciton annihilation.

At long time the decay of exciton population follows a power law $t^{-|\alpha|}$ with different asymptotic values of exponent. The values of decay exponent depend on amount of defect concentration in particular samples. It has been shown through Raman spectroscopy and XPS that CoMoCat SWNTs have more defects than previously studied HiPco SWNTs, and hence the decay exponent $(\alpha=0.30 \pm 0.01)$ is found to be lower than HiPco SWNTs $(\alpha=0.46 \pm 0.01)$ and exciton transport in CoMoCat is slower (sub-diffusive) than normal diffusive transport of excitons in a $1 \mathrm{D}$ system.

We also report a Monte Carlo simulation for random walk of particles on 1D lattice in the presence of potential barriers and found that the decay dynamics are consistent with hindered diffusion in a fluctuating potential landscape. Comparison of experimental results with numerical simulation shows a good agreement. From simulation, we found that as the number of defects increases, the asymptotic long time decay varies smoothly from normal diffusion to subdiffusion similar to the experimental results.

We also measured the exciton size of $(1.4 \pm 0.1) \mathrm{nm}$ in the case of CoMoCat1 SWNTs using the absorption cross section approach of $[71,72,133]$. This value of excitons size is comparable to previously reported value of exciton size in SWNTs [13, 14]. Reaction rate $k_{r} \approx(3.7 \pm 0.3) n m \cdot p s^{-1}$, subdiffusion rate $D_{\gamma} \approx(33 \pm 11) n m^{2} \cdot p s^{-0.54}$, diffusion coefficient $D=(8 \pm 1) \mathrm{nm}^{2} \cdot p s^{-1}$, and interaction length $r=(7.30 \pm 0.01) \mathrm{nm}$ are 
extracted from the fitting of experimental data with modified sub-diffusive long range model.

In short, the comparison of different SWNT samples is given in table 6-1. The exciton dynamics in CoMoCat2 sample appears to follow the power law $t^{-|\alpha|}$ with decay exponent approximately similar to CoMoCat1, and further Raman study of both samples shows almost similar number of defects despite the fact that CoMoCat2 was subject to more sonication. This shows that differences seem to be intrinsic to the synthesis method and does not depend on details of the processing. Even with vigorous processing, HiPco tubes have defect density less than CoMoCat nanotubes.

\begin{tabular}{|c|c|c|c|c|c|c|}
\hline \multirow[t]{2}{*}{ Sample } & \multirow[t]{2}{*}{ Preparation method } & \multirow{2}{*}{$\begin{array}{l}\text { Decay exponent } \\
\qquad \alpha\end{array}$} & \multicolumn{2}{|c|}{ Raman } & \multicolumn{2}{|l|}{ XPS } \\
\hline & & & $A_{D} / A_{G}$ & $\mathrm{I}_{\mathrm{D}} / \mathrm{I}_{\mathrm{G}}$ & $\begin{array}{c}c p^{3} \\
\text { FWHM (eV) }\end{array}$ & $\frac{A_{s p^{3}}}{A_{s p^{2}}}$ \\
\hline HiPco & DNA wrapping & $0.46 \pm 0.01$ & 0.26 & 0.09 & 2.76 & 1.00 \\
\hline CoMoCat1 & Dispersed in SC & $0.30 \pm 0.01$ & 0.48 & 0.21 & 2.82 & 1.44 \\
\hline
\end{tabular}

Table 6-1: Comparison of SWNT samples prepared through different synthesis methods.

Hence, optical probing of exciton dynamics is a contact-free diagnostic tool for studying the effects of defects on exciton transport in SWNTs. Comparison of SWNTs with different defect concentrations allows us to differentiate between intrinsic and extrinsic characteristics of the exciton transport. This will influence selection of nanotubes for electronic and photonic applications. 


\section{CHAPTER 7}

\section{CONCLUSION AND FUTURE WORKS}

This chapter is divided into three sections. In section 7.1, we present the major conclusions of the work presented in this thesis. The results of a number of exploratory studies where results were incomplete, inconclusive or negative are presented in section 7.2 for completeness and to indicate areas for further work. In section 7.3, we indicate some additional areas for future research.

\subsection{Conclusion}

We presented a range of optical measurements on different SWNT samples which illuminate the fundamental physics of 1D systems and are important in understanding their potential application in electronic and photonic devices especially in light emitting devices and non-linear optics.

Linear absorption measurements confirmed that the isolation of SWNTs can be achieved through DNA wrapping as well as through use of surfactants such as sodium cholate (SC). The isolation of SWNTs allowed us to identify the constituent species in a sample through comparison of peak positions observed in the linear absorption spectrum with that of previously tabulated energies [99]. An AFM study of HiPco SWNTs indicated that nanotubes are isolated due to helical wrapping by DNA. The average length measured with AFM indicates that the DNA-wrapped HiPco nanotubes were much shorter compared to CoMoCat samples. The presence of short nanotubes in the HiPco SWNT sample allowed us to see the single exciton regime in pump probe measurements.

Comprehensive Raman spectroscopy was performed to compare the defects in SWNTs synthesized through different processes (HiPco and CoMoCat). The results showed that the intensity of the defective band (D band) was significantly higher for the CoMoCat SWNTs, from which we inferred that the defect concentration was higher. The purity of our SWNT samples was verified via XPS which showed that no residual catalytic impurities were left after purification. The comparison of the XPS C1s spectra confirmed that the defect concentration in CoMoCat SWNTs was higher than HiPco SWNTs. 
We presented pump probe measurements of the decay of photogenerated excitons in a solution of semiconducting DNA-wrapped HiPco SWNTs in the regimes of many, few, and single exciton population and found that the exciton population decays through excitonexciton annihilation when more than one exciton is present on each nanotube. Under specific experimental conditions, exciton-exciton decay was found to correspond to an ideal 1D reaction-diffusion system. At sufficiently high exciton density, i.e. under high illumination intensity and at early times (100 $\mathrm{fs} \leq t \leq 3 \mathrm{ps)}$, a fast $t^{-1}$ power law decay was observed which can be explained by reaction-limited exciton-exciton annihilation. In this regime, the excitons are very close to each other and negligible transport is needed before the annihilation occurs. The rate of reaction is limited by the mean exciton concentration and reaction constant $k_{r}$. At longer times (10 ps $\leq t \leq 100 \mathrm{ps}$ ), exciton decay follows a approximately $t^{-1 / 2}$ power law regardless of optical excitation which is the characteristic of a diffusionlimited two body annihilation processes. In this regime, few excitons are left and they have to diffuse to find each other in order to annihilate. This regime is limited by the diffusive transport of excitons and depends on the diffusion coefficient $D$.

Exciton-exciton annihilation ceases at time $\sim 1 \mathrm{~ns}$, when excitons generated at the end of the nanotubes have sufficient time to diffuse to the centre and annihilate. From this information, the experimental signature corresponding to a single exciton per nanotube was determined which enabled us to calibrate the average number of excitons $N$ created per nanotube.

A fit to the experimental data in the different regimes yielded values for the reaction and diffusion coefficients. From the reaction-limited regime $(\mathrm{N}>14$ corresponding to an average exciton separation $<13 \mathrm{~nm}$ ), the reaction rate constant was found to be $k_{r}=(3.76 \pm 0.04) \mathrm{nm} / \mathrm{ps}$. In the diffusion-limited regime $(1<\mathrm{N}<14)$, a value of the diffusion coefficient was obtained which varied with the photoexcition density, probably as a result of laser-induced heating. This varied from $D=(3.6 \pm 0.6) \mathrm{nm}^{2} / \mathrm{ps}$ to $D=(8.1 \pm 0.4) \mathrm{nm}^{2} / \mathrm{ps}$ at the highest intensities. This latter value is close to the value of $\sim 10 \mathrm{~nm}^{2} / p s$ reported by Lüer et al. [13] in similar experiments. A saturation of exciton population was observed when the separation became comparable to the exciton spacing; we found an exciton size of $(1.26 \pm 0.08) \mathrm{nm}$ which is consistent with the value reported previously $[13,14]$.

The crossover between reaction-limited and diffusion-limited regimes with an abrupt and 
nonmonotonic dependence of effective reaction order on the exciton density was found to be poorly described by existing theories. A modified theory incorporating a finite reaction length provided an excellent fit to the experimental data for a reaction length of $\sim 7 \mathrm{~nm}$. This was interpreted as a result of medium-range dipole-dipole interactions.

We also provided the first experimental evidence of diffusive and sub-diffusive transport of excitons in quasi 1D SWNTs through studies of exciton annihilation dynamics in HiPco and CoMoCat SWNTs. We observed that excitons in HiPco SWNTs exhibit normal diffusive transport (where the long times dynamics of excitons follow approximately a $t^{-1 / 2}$ power law decay), whereas excitons in CoMoCat SWNTs decay more slowly as a result of subdiffusive transport. We associated this behaviour with a higher defect concentration in the CoMoCat sample through Raman and XPS study of these samples. The experimental results were compared to results from a Monte Carlo simulation of hindered 1D diffusion in a fluctuating potential landscape. The simulation showed that as the number of defects increased, the asymptotic long time decay varies smoothly from normal diffusion to subdiffusion similar to experimental results. Hence we have differentiated the intrinsic and extrinsic properties of exciton transport and decay on SWNTs.

We also measured the exciton size of $(1.4 \pm 0.1) \mathrm{nm}$ in CoMoCat SWNTs using absorption cross section approach of $[71,72,133]$. This value is comparable to previously reported value of exciton size in SWNTs [13, 14]. Reaction rate of $k_{r}=(3.7 \pm 0.3) n m \cdot p s^{-1}$, diffusion coefficient of $D=(8 \pm 1) n m^{2} \cdot p s^{-1}$, and subdiffusion coefficient of $D_{\gamma}=(33 \pm 11) n m^{2} \cdot p s^{-0.54}$ were extracted from the fitting of experimental data with simple modified sub-diffusive model.

\subsection{Exploratory Studies}

\subsubsection{Existence of Biexcitons}

A biexciton may be formed at high exciton density when two excitons are electrostatically bound [135]. The stability and possible signature of the biexcitons in SWNTs was reported by Pedersen et al. [136] in which they calculated their binding energy using an effective mass model. The binding energy of a biexciton depends on the radius of the nanotubes and is given by 


$$
E_{x x}(\mathrm{eV}) \approx \frac{0.0195 \mathrm{eV} \mathrm{nm}}{R_{n}[\text { in } \mathrm{nm}]}
$$

Here $R_{n}$ is the radius of carbon nanotubes.

The predicted signature of the biexciton in pump probe measurements is photoinduced absorption (PA) peak that is red shifted from the exciton resonance by the biexciton binding energy as shown in Fig. 7-1.

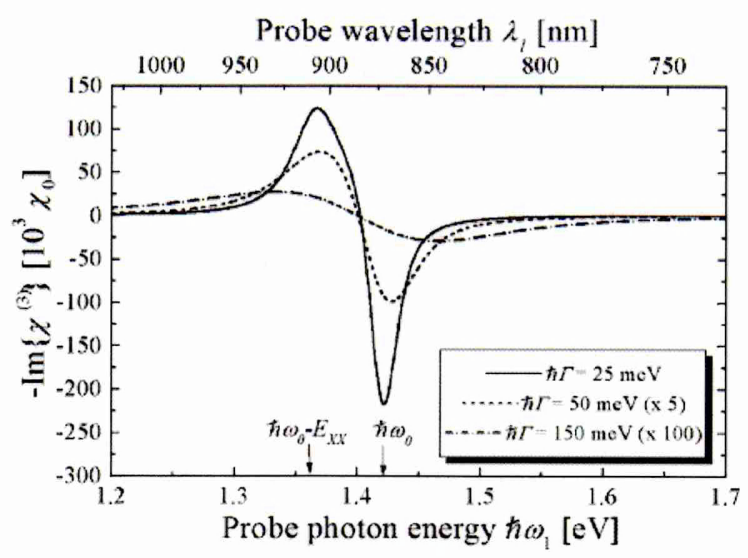

Figure 7-1: The signature of a biexciton assuming resonant pumping conditions and different values of inhomogeneous broadening, taken from Pedersen [136].

We have estimated the biexciton binding energy for our samples. The average diameter of the possible species excited at $1132 \mathrm{~nm}$ in our sample is $(0.84 \pm 0.05) \mathrm{nm}$ which corresponds to a biexciton binding energy of $\sim 46 \mathrm{meV}$, which is slightly greater than the thermal energy. In order to determine whether biexcitons are present in our sample, we performed spectrally resolved pump probe measurement by pumping the sample at $1132 \mathrm{~nm}$ and probing it with a white light probe pulse over the wavelength range of 900-1300 nm. The results are shown in Fig. 7-2 for zero time delay between the pump and probe pulses, where the transient absorption spectrum is plotted as a function of wavelength from $1050 \mathrm{~nm}$ to $1350 \mathrm{~nm}$.

According to our calculation, the expected wavelength to see the biexciton signature would be at $\sim 1178 \mathrm{~nm}$ but there is another peak at $1190 \mathrm{~nm}$ due to the presence of the $(11,3)$ nanotube species which makes it difficult to identify a biexciton signal in our present samples. There are three possible situations: (1) biexcitons are present but cannot be identified due to the presence of mixed chiralities of nanotubes, (2) biexcitons are present but 
cannot be identified due to the broad width of the spectral features in our samples which exceeds the biexciton binding energy, or (3) biexcitons are not present due to the fast excitonexciton annihilation process. Measurements on samples containing a single species of nanotube [30] with reduced inhomogeneous broadening will be required to determine whether biexcitons exist in SWNTs.

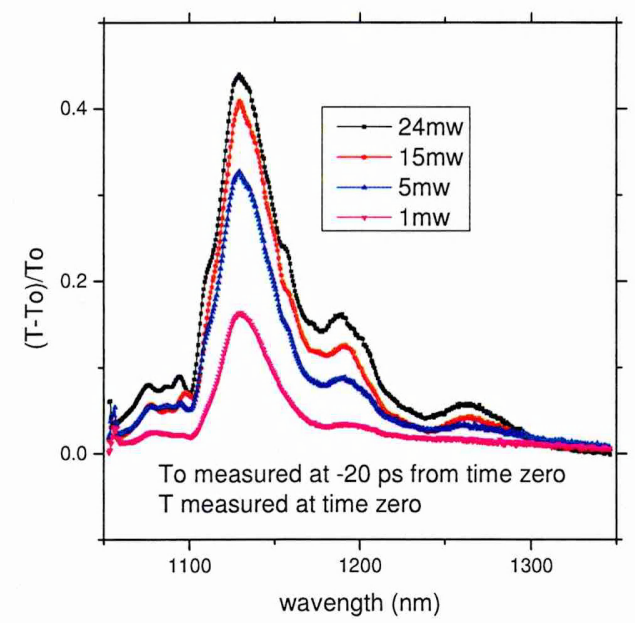

Figure 7-2: Spectrally resolved measurements of ss-DNA wrapped HiPco SWNTs plotted as function of wavelength. The sample was pumped at $1132 \mathrm{~nm}$ and probed with white light over the range of 1050-1350 $\mathrm{nm}$.

\subsubsection{Spectrally Resolved Pump Probe Measurements: Energy Transfer between Carbon Nanotubes}

The pump probe measurements reported previously in chapter 5 and 6 used a singlewavelength degenerate probe beam, allowing us to study the dynamical response of a single SWNT species at a time. Furthermore, in a previous study, Sutton [74] performed spectrally resolved measurements and reported that decay of the exciton population was independent of the tube diameter and chirality, and extracted a universal $t^{-1 / 2}$ asymptotic power law decay. On the other hand, it has been recently reported [137] that the exciton decay time decreases with decreasing the tube diameter due to strong exciton-phonon interactions in smaller diameter tube. Here, we report spectrally resolved measurements of the dynamical response of several species of DNA-wrapped HiPco SWNTs simultaneously, using a white light continuum probe. The method is similar to that of Sutton [74] except that an $1132 \mathrm{~nm}$ pump pulse (67 nm FWHM, resonant with the dominant exciton peak) was used, whereas Sutton 
used an $800 \mathrm{~nm}$ pump.

The spectrally resolved data is plotted in Fig. 7-3 (a), showing the pump induced transient spectrum as function of wavelength for the range of delays $\tau$. In Fig. 7-3 (b), the 2D contour plot of $\Delta \mathrm{T}(\lambda, \tau)$ obtained from spectra of Fig. 7-3 (a) is plotted. The measured quantity $\mathrm{S}(\tau, \lambda)$ is the normalised differential transmission $\Delta \mathrm{T} / \mathrm{T}_{0}$. In order to remove any background signal (arising especially from scattered pump light), the signal measured at a negative time of -5 ps was subtracted.
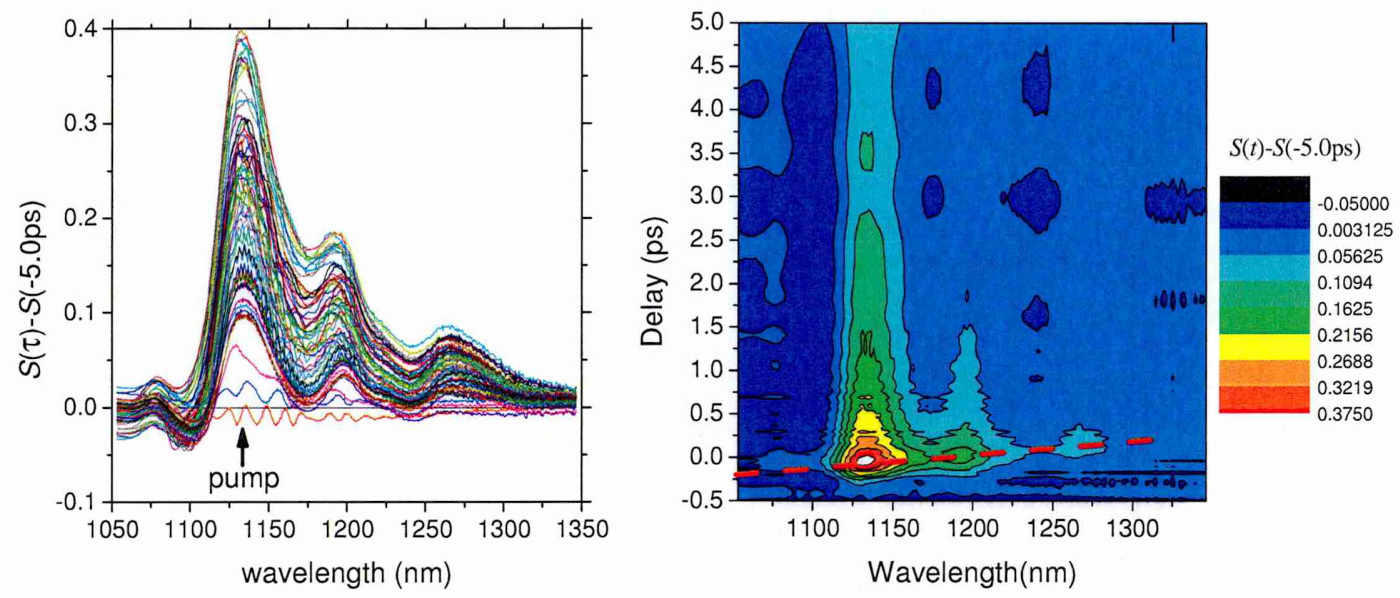

Figure 7-3: (a) Transient absorption spectrum of ss-DNA wrapped SWNTs in aqueous suspension as a function of wavelength for range of probe delays, $(b) 2 D$ contour plot of the spectrally resolved measurements.

The position of spectral features observed in Fig. 7-3 (a) exactly matches the peaks in the linear absorption spectrum (e.g. see Fig. 4-1). The first peak is excited resonantly at $1132 \mathrm{~nm}$ which creates the excitons on that particular nanotube species but excitons are also created on another two SWNT species as well (see Figure 7-3). We speculate on the mechanism for excitation of the SWNTs with excitonic resonances at $\sim 1192 \mathrm{~nm}$ and $1267 \mathrm{~nm}$ as follows.

1. The FWHM of the pump pulse is approximately $70 \mathrm{~nm}$. However if the pump intensity is high enough to strongly saturate the resonant exciton population, then there may be sufficient intensity which can excite other transitions far from the centre wavelength. This would excite nanotubes with exciton resonances both on the high and low wavelength sides of the $1132 \mathrm{~nm}$ centre wavelength. 
2. Energy transfer from the resonantly excited nanotubes to species with lower energy gaps may occur. Recently, resonant transfer of energy between DNA-wrapped SWNTs was observed and explained by Forster type electromagnetic near field coupling [129]. However, a resonant Forster transfer usually requires alignment of the ground state of the donor species with an absorbing state of the acceptor species, as well as close proximity of the donor and acceptor species.

3. As discussed above, there is significant broadband background absorption possibly due to absorption by metallic nanotubes or by plasmon resonances in graphitic impurities. This might provide a non-resonant coupling mechanism where carriers photogenerated by $1132 \mathrm{~nm}$ illumination rapidly lose energy and allow a coupling to lower-energy excitons. However a strong coupling to (gapless) metallic nanotubes or graphitic particles would be expected to provide a fast additional exciton quenching pathway, which is not observed experimentally.

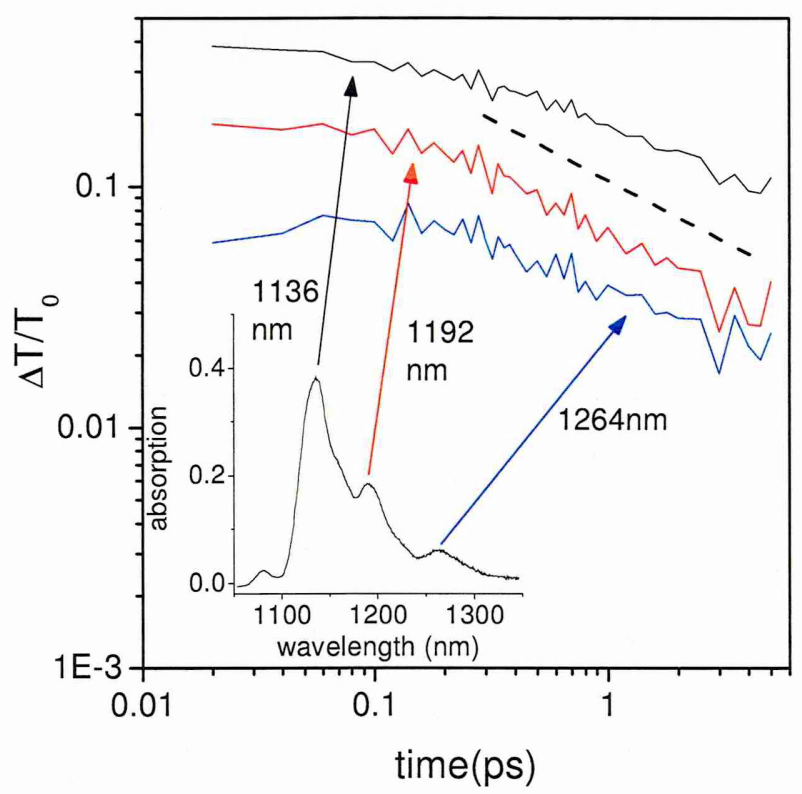

Figure 7-4: Dynamical response of HiPco SWNTs obtained through the vertical cut of Figure 7-3 (a). The dashed line shows a $t^{-1 / 2}$ decay.

Figure 7-3 (b) provides evidence of chirp in the pulse which can be seen at early delays (red line in the figure) and shows that the white light continuum is positive chirped, such that longer wavelengths lead the shorter. This should be accounted for in the extraction of dynamical behaviour. Decay curves obtained through a vertical cut of the spectra shown in 
Fig. 7-3 (a) are plotted in Fig. 7-4. It is difficult to extract the exact decay behaviour due to the low signal-to-noise ratio, especially at longer times; however on timescales of a few ps, a $t^{-1 / 2}$ power law decay seems to hold approximately.

Future work should identify the conditions required to resonantly excite a single nanotube species; this will then allow investigation of the mechanism for creation of exciton population on non-resonantly excited SWNT species. Also the measurement of different SWNT species is required for an investigation of how parameters such as exciton-exciton reaction coefficients, exciton diffusion coefficients, and exciton size depend on SWNT diameter and chirality.

\subsubsection{Annihilation Induced Heating}

As discussed in chapter 5 , the exciton population in diffusion-limited regime is determined by the diffusion coefficient $D$ and it was found that Different values of $D$ were required (e.g. see Fig. 5-9) for fitting of the results obtained at different pump intensities. We interpreted this as a result of photo-induced sample heating, which can be significant at these excitation levels. For example, local heating by photoexcited nanotubes has been investigated as a technique for destroying cancer cells [138].

During the exciton-exciton annihilation process, the recombination energy $\left(E_{11}\right)$ of one of the excitons is transferred to kinetic energy of the second exciton. This excess energy is very rapidly transferred to high energy phonons, which then decay into low energy phonon modes and ultimately increase the temperature of the system. In the case of the HiPco nanotubes studied in chapter 5, maximum of $N_{\max } \sim 150$ excitons were created by a single laser pulse. Hence a maximum total energy of $\left(N_{\max }-1\right) \times E_{11}$ is delivered to the phonon system of a single nanotube during the decay of these photogenerated excitons i.e. about $\sim 160 \mathrm{eV} / \mathrm{ns}$ $(26 \mathrm{nW})$ is delivered to the phonon system of a single nanotube from the annihilating excitons.

The transfer of energy (from exciton to excited exciton to non-equilibrium phonons to lowenergy phonons on a 'hot' nanotube to vibrations of the heated solvent) occurs on various timescales which have not yet been fully determined. We measured the degree of sample heating through its effect on the diffusion coefficient. By varying the pulse beam intensity and the laser repetition rate, it can be determined whether the changes are due to the peak 
laser intensity (i.e. independent of repetition rate) or the mean laser intensity.

The experiments were performed on the solutions of CoMoCat SWNTs described in chapter 6. Figure 7-5 shows the transient transmission taken at 4 different repetition rates. The beam intensity was varied to maintain the peak pulse amplitude approximately constant i.e. to keep constant the number of excitons created per nanotube per pulse. The decay curves shows the distinct regions of normal and anomalous reaction and diffusion as described in section 6.2. The upper traces are normalised to 1 ; the similarity of the traces at early times is an indication of the constant exciton generation. However the long term asymptotic behaviour shifts in amplitude with the laser repetition rate (i.e. with the mean laser power). Similar results were obtained when the average laser intensity was lowered by a factor of 2 (part (b) of the figure).
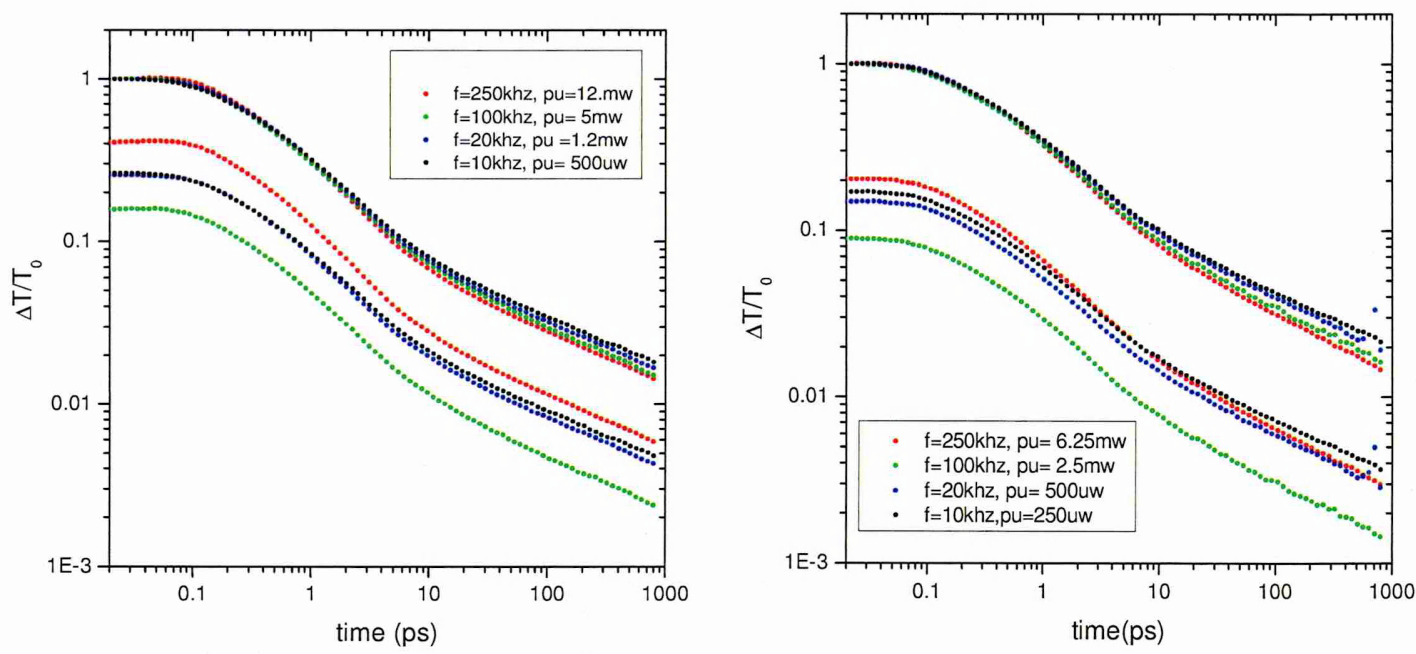

Figure 7-5: (a) Differential transmission for 4 different laser repetition rates. The laser intensity was adjusted to give approximately constant peak pulse amplitude. Pump intensity at the highest repetition rate was $12 \mathrm{~mW}$. (b) Same data but for a reduced average laser intensity.

From the results shown in Fig. 7-5, we conclude that the diffusion coefficient depends on the mean illumination intensity (i.e. simple heating of the sample), and not on the photogenerated exciton density per pulse. This is consistent with previous reported timescales for energy transfer, for example Song et al. [139] reported that decay of high energy phonons to acoustic phonons in equilibrium with the lattice occurs on a timescale of a few picoseconds and Huxtable et al. [140] reported that transfer of energy to the solvent occurs within 10-20 ps. Hence the thermal equilibration seems to occur on short timescales where the 
reaction rate is limited by the reaction probability, not by the transport of reactants. Future measurements at lower excitation density might allow determination of the diffusion coefficient at times $<10$ ps after photoexcitation; when a strongly non-equilibrium phonon system is present, the deviations from the equilibrium value is expected at sufficiently short times.

\subsubsection{Measurement of Stretch-aligned Carbon Nanotubes}

The optical response of SWNTs is highly anisotropic due to their quasi-1D topology. However this anisotropy is not obvious in typical as-synthesized samples due to the random orientation of nanotubes. Considerable effort has been made to produce structures with aligned nanotubes, by means of mechanical stretching of CNT-polymer composites [141146], spun composite fibres [147], application of a magnetic field [72, 148], CVD growth of vertically-aligned SWNTs [149, 150], electrical-field assisted thermal annealing [151], and formation of liquid crystals in certain solvents [152]. These growth methods are reviewed by Iakoubovskii (2009) [153]. These aligned nanotubes structures are needed because of their wide range of application including optical polarisers at frequencies from the visible [146] to THz [154-156], saturable absorbers for mode-locking in lasers [157], and as loudspeaker diaphragms [158]. The physics and applications were also recently reviewed by Lan et al. (2011) [159].

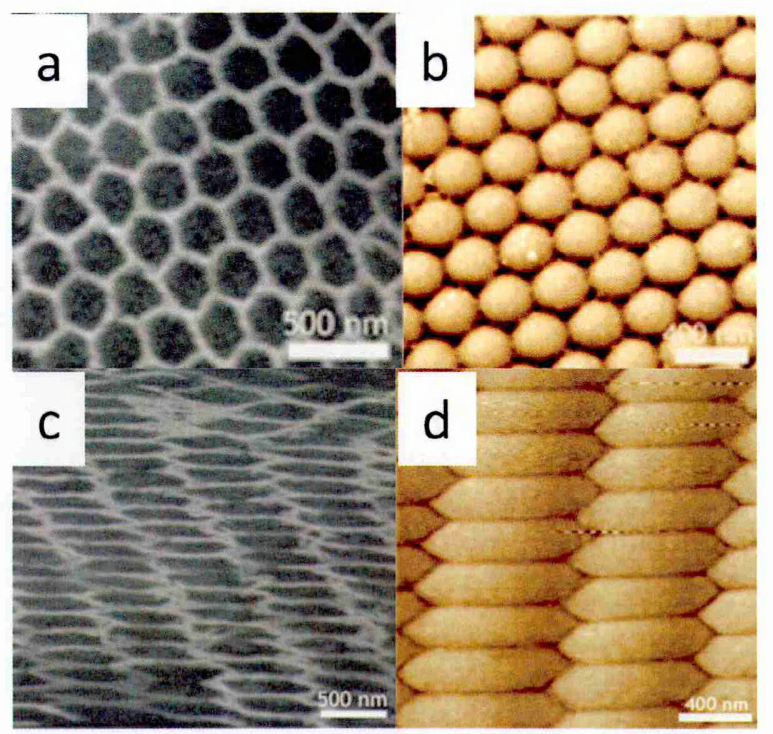

Figure 7-6: Transmission electron micrographs $(a, c)$ and phase contrast atomic force micrographs $(b, d)$ of unstretched $(a, b)$ and $100 \%$ strained $(c, d)$ samples. Figure taken from Jurewicz et al. [96]. 
We studied exciton annihilation dynamics on stretch-aligned nanotubes (which is expected to allow control of the intertube coupling). A significant intertube coupling was expected in the unstretched samples, which would decrease on stretching leading to a slower decay of photoexcited states. The samples, in the form of thin films with low optical transmission, were provided by Dr. Izabela Jurewicz and Dr. Alan Dalton of the Department of Physics at the University of Surrey, and are described in section 4.2.4. Micrographs of the unstretched and $100 \%$ strained samples are shown in Fig. 7-6 [96].

Degenerate pump probe measurements were performed at a wavelength of $1170 \mathrm{~nm}$ with a laser repetition rate of $250 \mathrm{kHz}$. A stretched (150\%) sample was rotated so that the nanotubes were aligned parallel or perpendicular to the electric field vector of the pump pulse. The pump probe results are plotted in Fig. 7-7 (a) for a $10 \mathrm{~mW}$ pump beam, showing a maximum signal for the case of parallel orientation of nanotubes with the laser polarisation and a minimum when the nanotubes are perpendicular to the laser polarisation. These results indicate a strong anisotropy and also confirm that the nanotubes are aligned in one direction upon stretching [96]. Bommeli et al. [160] also showed the anisotropic properties of metallic nanotubes.
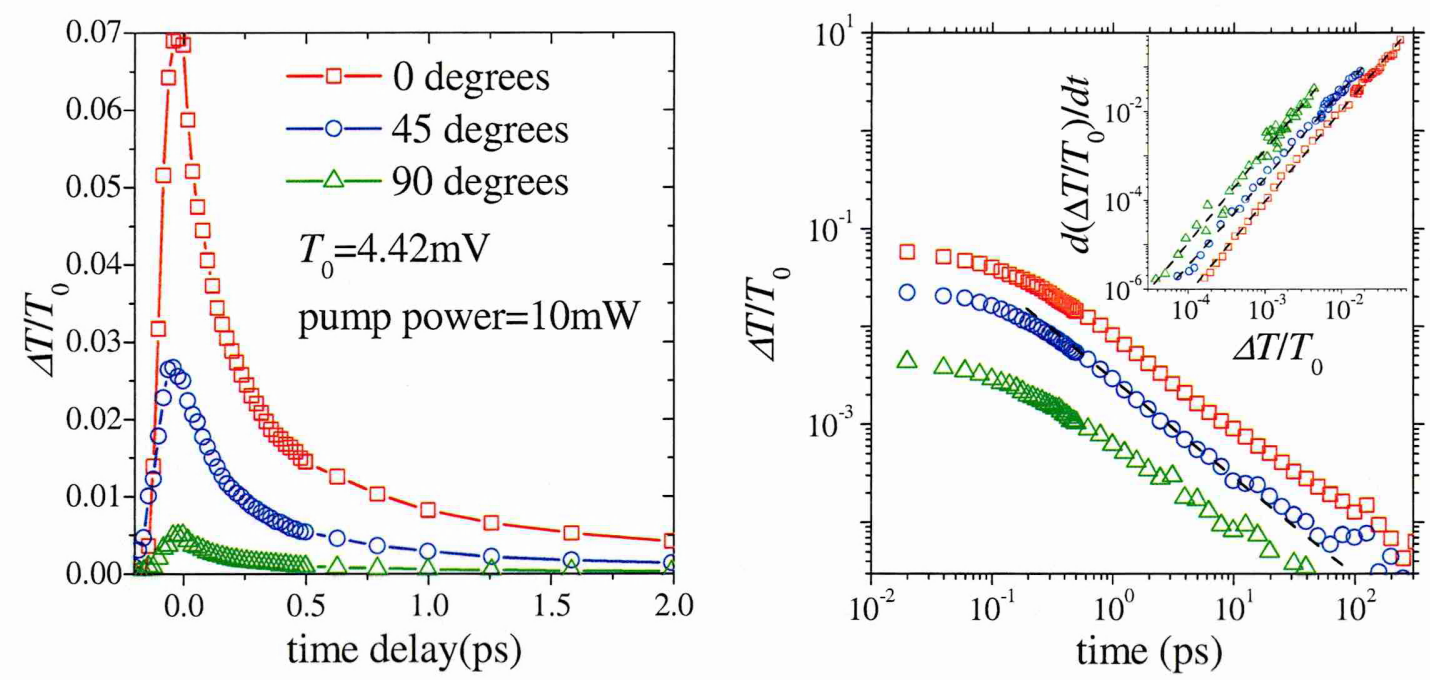

Figure 7-7: (a) Pump probe measurement of anisotropy of saturable absorption and its decay in a stretched sample [150\%]. The differential transmission is maximum in the case of parallel orientation of nanotubes with laser beams (red curve) and minimum when they are orthogonal (green curve). (b) Log-Log plot of the photoexcitation, which decays according to a $t^{-1}$ power law (dashed line). Inset shows the reaction rate which is proportional to the square of the exciton population (dashed line). 
In Fig. 7-7 (b), the log-log plot of the differential transmission is shown. This figure shows that for times $>0.5 \mathrm{ps}$, the photoexcited excitons decay according to a $t^{-1}$ power law for all cases. This is also confirmed by plotting the derivative $d\left(\Delta T / T_{0}\right) / d t$ against $\Delta T$ (see inset of Fig. 7-7 (b)). The logarithmic gradient of 2 (dashed line) in the figure is an indication of reaction-limited exciton-exciton annihilation. This reaction-limited region persists over a wider range of times and densities compared to the results on solutions presented in chapter 5. We attributed this to heating effects that leads to an enhancement of the diffusion coefficient and hence suppresses the crossover to a diffusion-limited regime. The heating effect in film is stronger compared to solution phase because of the presence of a higher nanotube concentration as well as possible absence of convective heat dissipation. As a result, the maximum optical densities employed for the SWNT solutions can cause irreversible damage to the SWNT composite films.
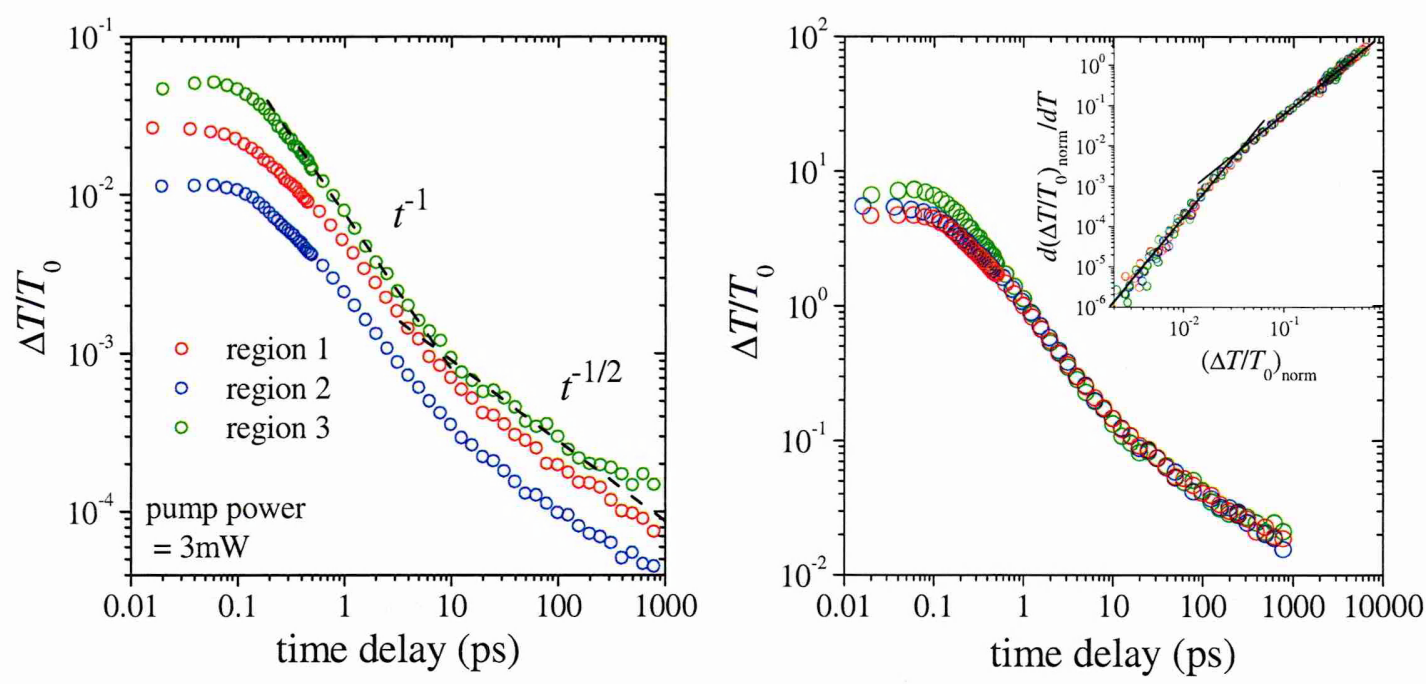

Figure 7-8: (a) Pump probe measurement of a stretched sample at 3 different locations. (b) Data normalised at delay time of 2 ps. Inset: derivative of differential transmission plotted against differential transmission on a log-log scale. The gradient is the reaction order which is equal to 2 and 3 (dashed lines) at high and low density regimes respectively.

The pump power was reduced to $3 \mathrm{~mW}$ and pump-probe measurements were performed on a stretched $(150 \%)$ SWNT sample. The data, which was collected from three different regions of the sample, is characterised by two distinct regions, with a $t^{-1 / 2}$ power law at times $>10$ ps and a faster decay (approaching $t^{-1}$ ) at shorter times (Figure 7-8 (a)). The curves were normalised at a delay time of 2 ps and are shown in Fig. 7-8 (b). These curves 
are identical after times $>2$ ps for all cases. However, small variations in the initial excitation density were observed at early times which may be due to inhomogeneities in the local SWNT concentration. A log-log plot of the derivative is shown in the inset of Fig. 7-8 (b) which indicates a clear transition between reaction-limited and diffusion-limited regimes. The behaviour observed here is remarkably similar to that reported in chapter 5 for ss-DNA wrapped HiPco SWNTs.

Figure 7-9 shows the decay exponent $\alpha$ obtained from the logarithmic derivative of Figures 7-7 (b) and 7-8 (a). For the case of $3 \mathrm{~mW}$ pump beam, the minimum value of $\alpha$ is $\alpha \sim-0.9$ and the asymptotic behaviour of $\alpha=-0.5$ is reached at times $>100$ ps. For a $10 \mathrm{~mW}$ pump power, the cross-over from reaction- limited to diffusion-limited behaviour occurs at around $100 \mathrm{ps}$, and the asymptotic regime is not reached. However a value of $\alpha=-1$ is observed at a delay time of $1 \mathrm{ps}$, corresponding to the first observation of a true $t^{-1}$ decay in the raw transient absorption data of Fig. 7-7 (b).

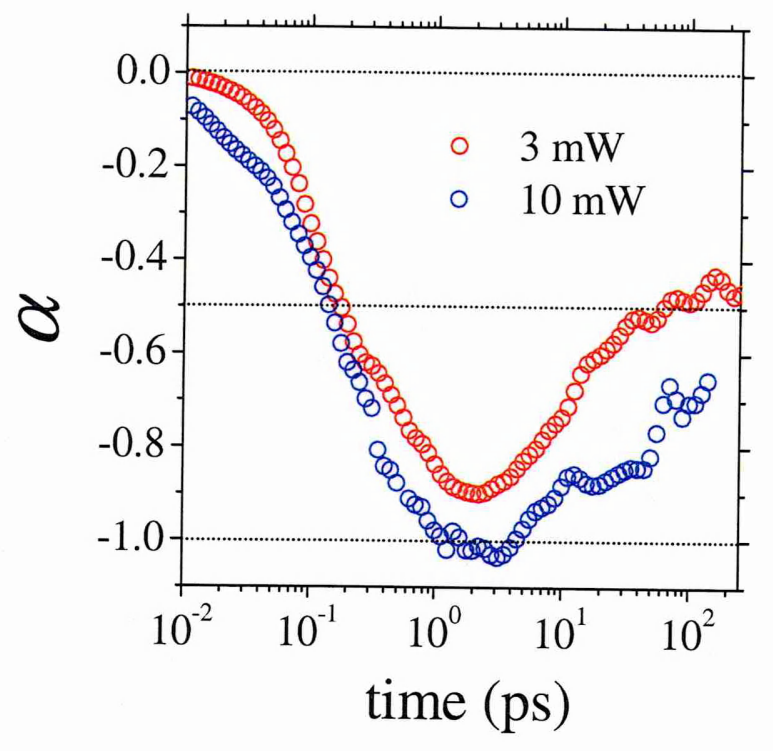

Figure 7-9: The decay exponent $\alpha$ for pump beam intensities of $3 \mathrm{~mW}$ and $10 \mathrm{~mW}$. The $3 \mathrm{~mW}$ data is the average of the 3 curves shown in Fig. 7-8 (a).

The average exciton density on a nanotube could not be determined in this sample because (1) an estimation of the exciton density cannot be made since the density of nanotubes was not sufficiently known, (2) the regime of a single exciton per nanotube was not observed within our experimental time as the nanotubes were sufficiently long, and (c) the saturation of the exciton density was not seen because sample damage limited the maximum 
photoexcitation level.

Prior to carrying out the dynamical measurements, all of the available samples were stretched and relaxed. According to Jurewicz et al. [96], the electrical conductivity recovers to $\sim 40 \%$ of its original value following an applied strain of $200 \%$. Some changes to the morphology are visible in the electron micrographs, as the latex relaxes to its initial position while carbon nanotubes do not. Hence there is a change in the connectivity of the nanotube network during the stretch / relax cycle, although it is reported as smaller that for disordered nanotube composites presumably due to the reduced entanglement [161]. Individual nanotubes suspended in a polymer have been shown to buckle as a result of stretch and release cycles [162]. Changes in the connectivity of the nanotubes are expected to have significant effects on the exciton dynamics.

To investigate the effect of stretch and release cycles on the exciton dynamics, pump probe measurements were performed on stretched and unstretched samples. For this purpose, SWNT sample was stretched (150\%) and unstretched many times and each time a pump probe transient was measured. For the stretched samples, the transients were similar to those shown in Fig. 7-8. For the unstretched samples, the differential transmission $\left(\Delta T / T_{0}\right)$ taken on two different days is plotted in Fig. 7-10 (a) in which the blue curve is obtained after a single stretch-release cycle, whereas the red trace is recorded after three cycles. These traces together with the data from stretched samples shown in Fig. 7-8 (a) are replotted in Fig. 7-10 (b), where the curves have been shifted in time to account for the initial conditions, and have been normalised at a time delay of 1 ps. The exciton dynamics in all cases are identical. This independence on stretching, and the slow decay of the exciton population, shows that the SWNTs are isolated from each other.

In order to investigate the sample which had not been previously stretched, we prepared a new sample by drop-casting a similarly-prepared SWNT-polymer solution onto a mica sheet. The pump probe measurement was performed at same wavelength of $1170 \mathrm{~nm}$. The transient differential transmission is shown in Fig. 7-11 (a). The figure shows that the measured behaviour is dramatically different from the stretched samples and is consisting of a fast quasiexponential decay superimposed on a long-lived negative (induced absorption) signal. In Fig. 7-11 (b), the absolute value of differential transmission is fitted to a simple sum of exponential decays with time constants of 0.25 ps and $1.6 \mathrm{ps}$, with an offset representing the slowly-decaying negative signal. 

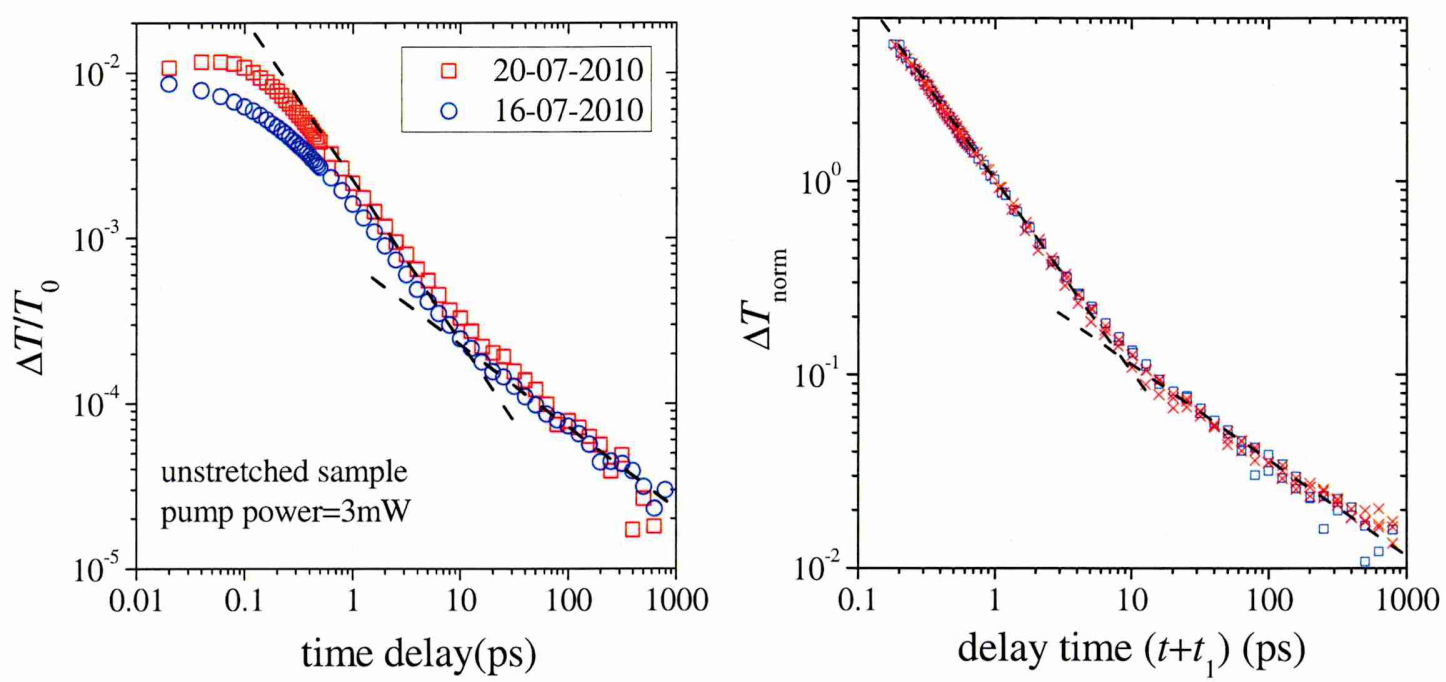

Figure 7-10: (a) Pump-probe transients taken on unstretched sample before (16/7/2010) and after (20/7/2010) multiple stretches by 150\%. (b) Transient response of stretched (red crosses, data from Fig. 7.8 (a)) and unstretched (blue squares; data from Fig. 7-10(a)) samples, normalised at a delay of 1 ps. The time axis is shifted to account for the initial condition.

These decays times are typical of values reported in metallic nanotubes or graphitic materials. Hence this observed behaviour suggests that excitons transfer rapidly from the semiconducting SWNTs to metallic nanotubes or graphitic impurities, where they decay rapidly. This kind of behaviour has been previously reported in photoexcited bundles of SWNTs by Hashimoto et al. [149], where a two-component decay with time constants of 140 fs and 560 fs was observed for both polarisations. In addition to these, an additional component with $\sim 1$ ps decay was observed with excitation polarised perpendicular to the bundles, and was attributed to coupling with surface plasmons.

There are two possible interpretations of the very different dynamical behaviours shown in Figures 7-8 and 7-11.

(1) The dropcast sample has similar characteristics to the films, before the films are stretched for the first time. In this case nanotubes are in intimate contact. The photoexcitation of semiconducting SWNTs generates excitons which transfer rapidly between nanotubes. These excitons decay rapidly when they transfer to a metallic nanotube. However, this intimate contact is broken to a sufficient degree on stretching 
the sample for the first time, and the decay of excitons on semiconducting SWNTs is limited by exciton-exciton annihilations rather than inter-tube transfers. On relaxing the sample, the intimate contact between nanotubes is not re-established. Hence the nanotubes remain essentially isolated from each other. However in this case one might expect a larger change in the sample conductivity than was reported by Jurewicz et al. [96].

(2) The dropcast sample had different properties to the films prior to stretching. The SWNTs in dropcast are not well isolated due to either insufficient sonication or other factors. The SWNTs in the as-made composite films are isolated from each other, and this property is robust to stretching-relaxation cycles.

To differentiate between these two interpretations, further measurements are required on fresh samples which have not been pre-stretched, with the dynamical properties recorded as the samples are stretched in situ.
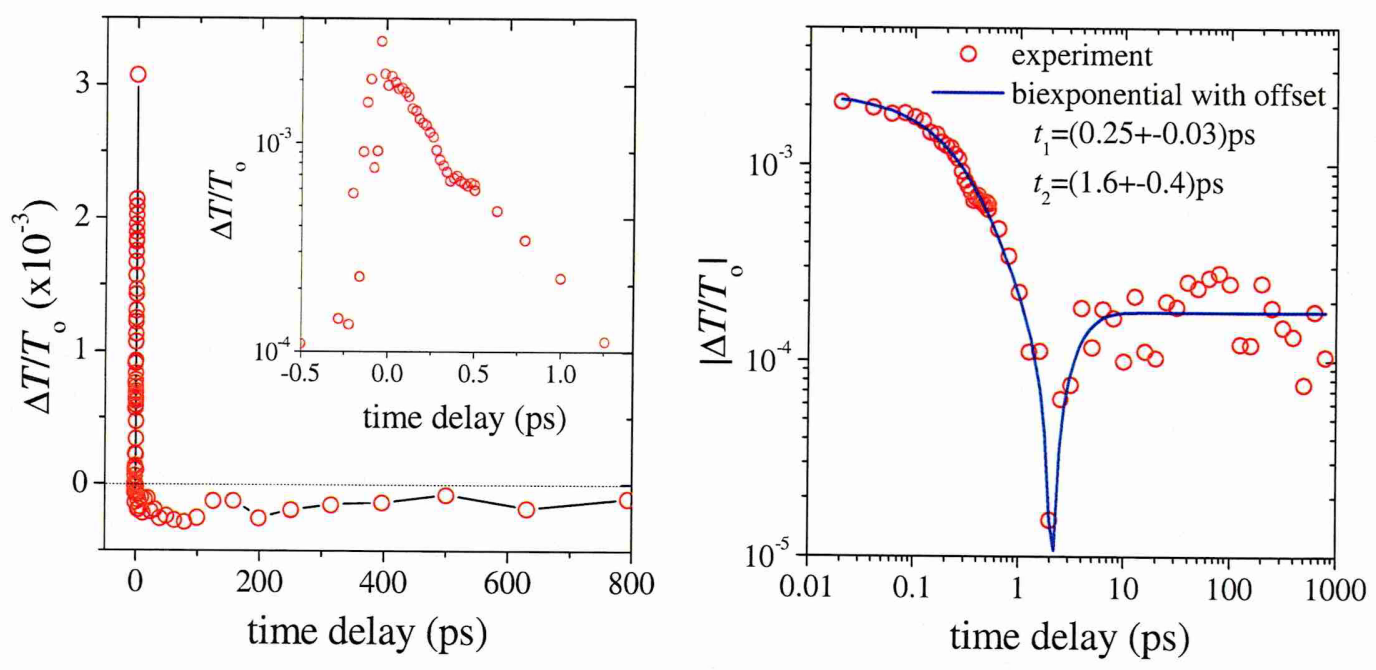

Figure 7-11: Pump probe data taken on drop-cast SWNT-polymer (a) Linear plot showing fast initial decay followed by a long-lived negative signal. Inset: log-linear plot of the first ps, indicating an approximately exponential decay. (b) Absolute value of differential transmissivity, fitted to a biexponential decay with a constant offset.

In conclusion, strong anisotropy of the optical absorption of a stretch-aligned ordered SWNT-polymer composite was observed, where a maximum magnitude of signal was observed in the case of parallel orientation of laser beam to nanotube and a minimum in the case of orthogonal orientation. Under high excitation, the decay rate of the photogenerated 
excitons was dominated by exciton-exciton annihilation, with distinct regions of reactionlimited and diffusion-limited behaviours. The reaction kinetics was identical for stretched and unstretched samples, and was very similar to those of SWNTs in solution, in spite of the very different environment. These results show that the SWNTs are essentially isolated in stretched samples and in samples which were allowed to relax after stretching. In the case of an unstretched dropcasted sample, a much faster decay processes was observed which is suggestive of rapid inter-tube transfer in bundled nanotubes. However this result was so far inconclusive since the 'fast' and 'slow' behaviours were not observed in a single sample before and after stretching.

\subsubsection{Effects of Spatial Ordering on Early Reaction Kinetics - Detectability of the 'Exciton Crystal'}

Doering and Ben-Avraham [55] calculated the reaction kinetics for different initial spatial distributions for the 1D coalescence-diffusion reaction for the case of efficient reactions $(p=1)$. They found that the initial reaction rate was decreased for spatially-ordered states, whereas it was enhanced for random distributions, compared to the predictions of the simple rate equations. They performed the calculations using both an exact analytical model and Monte Carlo simulations. There is apparently no experimental confirmation of this phenomenon to date.

Measurements of the early dynamics in HiPco SWNTs (presented in chapter 5) under various photoexcitaion densities showed a clear deviation from the rate equations at early times. The time- derivative of pump probe transients is shown in Fig.7-12 (a). The decay rate decreases for the case of highest pump intensity where the exciton density is saturated, while at lower intensity the decay rate exceeds the predictions of the power law. We associated the exciton saturation with Pauli exclusion, where excitons cannot overlap and possibly formed an ordered structure with equal separation. Monte Carlo simulations (performed by Prof J. Allam) performed with similar parameters chosen to fit the experimental behaviour, showed similar deviations from the power law at early times when the initial spatial distribution was either random or spatially-ordered (Fig. 7-12 (b)). We initially believed these results represented an experimental observation of spatial ordering named as 'exciton crystal' in a saturated exciton distribution. Ivanov and Haug [163] had predicted the existence of a similar state, and had predicted the emission of super-radiance at low temperatures. 
The Monte Carlo simulations were performed assuming short-range exciton-exciton interactions. In this case the transition between reaction-limited and diffusion-limited states is very gradual, and there is still a diffusion-limited contribution even at the highest exciton population. It was found that this contribution was responsible for the weak dependence on initial spatial states seen in Fig. 7-12 (b). When simulations were repeated with longer range interactions, the early dynamics were entirely reaction-limited independent of the interparticle spatial distribution. Hence other explanations must be found for the behaviour given in Fig. 7-12 (a).
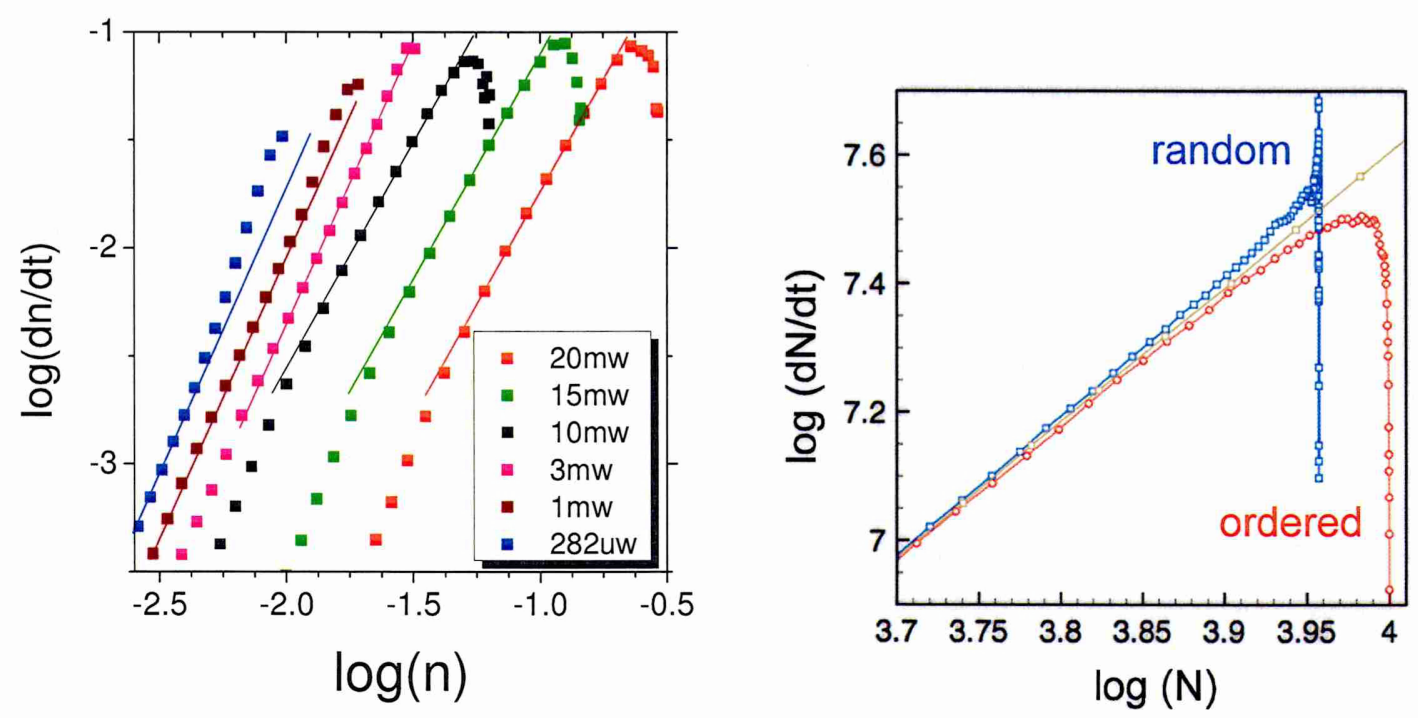

Figure 7-12: (a) Measured reaction rates for different photoexcitation levels. The curves have been shifted laterally for clarity. (b) Monte Carlo calculation of the reaction rate for random and spatially-ordered initial states.

The deviations from power law behaviour occur at times below $\sim 300$ ps which is apparently longer than the pulse duration. However, it is possible that there is still some generation of particles at this time because of strong saturation, which can explain the negative deviation from the power law. The positive deviation at lower excitations may be due to spatial variation in the excitation. This is unavoidable if finite light absorption occurs, and leads to different reaction kinetics. The considerations underline the usefulness in performing measurements under conditions where saturation ensures spatial uniformity of the excitation.

Note that there is possibility that spatial-ordering may still be present as a result of the 
Pauli blocking, however, in the reaction-limited regime it cannot be observed through the reaction kinetics.

\subsection{Further Suggestions for Future Work}

1. A deviation from the $t^{-1 / 2}$ power law decay at times approaching $\sim 1 \mathrm{~ns}$ was observed in our pump probe data of HiPco SWNTs (discussed in chapter 5) which we attributed to the presence of a single exciton per nanotube. This single exciton left on the nanotube will decay radiatively or non-radiatively, but the exact decay mechanism is not known and measurements with delay $>1$ ns were not possible with the available apparatus. Measurements of the exciton decay on longer timescales would allow an investigation of these radiative and non-radiative processes.

2. A very notable feature of our exciton annihilation results is that there is no evidence for additional non-radiative exciton decay mechanisms on timescales up to $1 \mathrm{~ns}$. This is in apparent contradiction to measurements of decay of the PL where decay times of $\sim 10-100$ ps (much shorter that the radiative lifetime of $\sim 1 \mathrm{~ns}$ ) were observed due to exciton quenching at defects[40,41,49, 164-166]. We postulate that at the high photoexcitation levels in our experiments, traps are filled immediately (i.e. within the duration of the pump pulse), and that detrapping times are long compared with exciton annihilation times. Hence the traps play no further role in the decay of the exciton dynamics. However, role of traps on the exciton dynamics can be determined by experiment with three laser pulses in which one strong pump pulse will excite the sample and will fill the available traps and then after delay of $1 \mathrm{~ns}$ second (when exciton-exciton annihilation finished), a weak second pump pulse will be sent and dynamics will be probed with third probe pulse.

3. As discussed in chapter 5 , the reaction probability is simply the ratio of diffusion time $\left(t_{0}\right)$ and reaction time $\left(t_{1}\right)$ i.e.

$$
p=\pi\left(\frac{t_{0}}{t_{1}}\right)
$$

Hence it can be directly measured experimentally using coherent technique (e.g. FWM) in which $t_{0}$ is annihilation induced contribution to dephasing time and $t_{1}$ is population decay time (1/population relaxation rate). 
4. The loss of coherence (dephasing) of excitons occur as a result of the exciton-exciton scattering, exciton-phonon scattering. In the case of exciton-phonon scattering, the momentum of exciton relaxes and hence changes the phase. However, for energy relaxation, exciton-phonon interaction has to occur many times because of large momentum and small energy of the phonon. This dephasing time can be measured from the homogenous spectral line width using the relation

$$
\Gamma=\frac{2 \hbar}{T_{d p h}}
$$

where $T_{d p h}$ is depahsing time. We measured the value of diffusion time ( $t_{0}=32 \mathrm{fs}$ ) which is much smaller that the reported dephasing time measured with four wave mixing experiment (FWM) and PL spectroscopy. Future PL experiment on same DNA wrapped HiPco sample is required to compare the dephasing time with measured diffusion time.

5. As discussed in chapter 2, a range of values of diffusion coefficient were reported using different techniques. We measured much smaller value of $\mathrm{D}\left(0.1 \mathrm{~cm}^{2} / \mathrm{s}\right)$ from pump probe experiment as compared to PL experiments, where the large value of $D$ (upto $100 \mathrm{~cm}^{2} / \mathrm{s}$ ) was reported. A simultaneous measurement of PL quenching experiment on same DNA wrapped HiPco sample is required to get diffusion coefficient in order to compare the large discrepancy between values of $D$ obtained from two different techniques.

6. The effect of non-uniform illumination was observed in our experimental data which was unavoidable in our current experimental setup. In order to remove its contribution, a measurement with spatial uniformity of the excitation is required on a thin film sample with dots of carbon nanotubes.

7. In theory, we used $\frac{\overline{x^{2}}}{\bar{x}^{2}}=\frac{4}{\pi}$ (a quantity which characterises the interparticle distribution function) which is true for short range $(r=\Delta z)$ efficient reactions $(p=1)$. However for $r>\Delta z$ and $p<1$, this quantity $\overline{x^{2}} / \bar{x}^{2}$ should be further modified. This is proposed future work for Monte Carlo simulations. 


\section{REFERENCES}

[1] S. Iijima, T. Ichihashi, Single-shell carbon nanotubes of 1-nm diameter, Nature 363 (1993) 603605.

[2] R. Saito, G. Dresselhaus, M.S. Dresselhaus, Physical properties of carbon nanotubes, World Scientific, 1998.

[3] F. Wang, G. Dukovic, E. Knoesel, L.E. Brus, T.F. Heinz, Observation of rapid Auger recombination in optically excited semiconducting carbon nanotubes, Phys. Rev. B, 70 (2004) 241403.

[4] R. Saito, G. Dresselhaus, M. Dresselhaus, Trigonal warping effect of carbon nanotubes, Phys. Rev. B, 61 (2000) 2981-2990.

[5] S. Datta, Quantum transport: atom to transistor, Cambridge University Press, 2005.

[6] A. Javey, J. Kong, Carbon nanotube electronics, Springer, 2009.

[7] D. Tomanek, Introduction and theoretical background, Michigan State University, USA.

[8] E. Chang, G. Bussi, A. Ruini, E. Molinari, Excitons in carbon nanotubes: An ab initio symmetrybased approach, Phys. Rev. Lett., 92 (2004) 196401.

[9] C.D. Spataru, S. Ismail-Beigi, L.X. Benedict, S.G. Louie, Excitonic effects and optical spectra of single-walled carbon nanotubes, Phys. Rev. Lett., 92 (2004) 77402.

[10] V. Perebeinos, J. Tersoff, P. Avouris, Scaling of excitons in carbon nanotubes, Phys. Rev. Lett., 92 (2004) 257402.

[11] F. Wang, G. Dukovic, L.E. Brus, T.F. Heinz, The optical resonances in carbon nanotubes arise from excitons, Science, 308 (2005) 838-841.

[12] R. Waiser, Nanoelectronics and Information Technology: Advanced Electronic Materials and Novel Devices, in, Wiley-VCH, , 2005.

[13] L. Luer, S. Hoseinkhani, D. Polli, J. Crochet, T. Hertel, G. Lanzani, Size and mobility of excitons in $(6,5)$ carbon nanotubes, Nat. Phys., 5 (2009) 54-58.

[14] R.B. Capaz, C.D. Spataru, S. Ismail-Beigi, S.G. Louie, Diameter and chirality dependence of exciton properties in carbon nanotubes, Phys. Rev. B, 74 (2006) 121401.

[15] J. Maultzsch, R. Pomraenke, S. Reich, E. Chang, D. Prezzi, A. Ruini, E. Molinari, M. Strano, C. Thomsen, C. Lienau, Excitons in carbon nanotubes, physica status solidi (b), 243 (2006) 32043208.

[16] T.G. Pedersen, Variational approach to excitons in carbon nanotubes, Phys. Rev. B, 67 (2003) 073401.

[17] H. Zhao, S. Mazumdar, Electron-electron interaction effects on the optical excitations of semiconducting single-walled carbon nanotubes, Phys. Rev. Lett., 93 (2004) 157402.

[18] J. Maultzsch, R. Pomraenke, S. Reich, E. Chang, D. Prezzi, A. Ruini, E. Molinari, M. Strano, C. Thomsen, C. Lienau, Exciton binding energies in carbon nanotubes from two-photon 
photoluminescence, Phys. Rev. B, 72 (2005) 241402.

[19] G. Dukovic, F. Wang, D. Song, M.Y. Sfeir, T.F. Heinz, L.E. Brus, Structural dependence of excitonic optical transitions and band-gap energies in carbon nanotubes, Nano Lett., 5 (2005) 2314-2318.

[20] Y.Z. Ma, L. Valkunas, S.M. Bachilo, G.R. Fleming, Exciton binding energy in semiconducting single-walled carbon nanotubes, The Journal of Physical Chemistry B, 109 (2005) 15671-15674.

[21] P. Nikolaev, M.J. Bronikowski, R.K. Bradley, F. Rohmund, D.T. Colbert, K. Smith, R.E. Smalley, Gas-phase catalytic growth of single-walled carbon nanotubes from carbon monoxide, Chemical physics letters, 313 (1999) 91-97.

[22] S. Reich, C. Thomsen, P. Ordejon, Electronic band structure of isolated and bundled carbon nanotubes, Phys. Rev. B, 65 (2002) 155411.

[23] M.J. O'connell, S.M. Bachilo, C.B. Huffman, V.C. Moore, M.S. Strano, E.H. Haroz, K.L. Rialon, P.J. Boul, W.H. Noon, C. Kittrell, Band gap fluorescence from individual single-walled carbon nanotubes, Science, 297 (2002) 593-596.

[24] S.M. Bachilo, M.S. Strano, C. Kittrell, R.H. Hauge, R.E. Smalley, R.B. Weisman, Structureassigned optical spectra of single-walled carbon nanotubes, Science, 298 (2002) 2361-2366.

[25] O. Matarredona, H. Rhoads, Z. Li, H. Jeffrey, L. Balzano, D.E. Resasco, Dispersion of singlewalled carbon nanotubes in aqueous solutions of the anionic surfactant NaDDBS, The Journal of Physical Chemistry B, 107 (2003) 13357-13367.

[26] D. Mersch, Separation of single-walled carbon nanotubes by electric type, in: Department of Physics, University of Surrey, 2009.

[27] J. Foster, S. Singamaneni, R. Kattumenu, V. Bliznyuk, Dispersion and phase separation of carbon nanotubes in ultrathin polymer films, Journal of Colloid and Interface Science, 287 (2005) 167172.

[28] Q.H. Yang, N. Gale, C.J. Oton, F. Li, A. Vaughan, R. Saito, I.S. Nandhakumar, Z.Y. Tang, H.M. Cheng, T. Brown, W.H. Loh, A Raman probe for selective wrapping of single-walled carbon nanotubes by DNA, Nanotechnology, 18 (2007).

[29] M. Zheng, A. Jagota, E.D. Semke, B.A. Diner, R.S. McLean, S.R. Lustig, R.E. Richardson, N.G. Tassi, DNA-assisted dispersion and separation of carbon nanotubes, Nature materials, 2 (2003) 338-342.

[30] X. Tu, S. Manohar, A. Jagota, M. Zheng, DNA sequence motifs for structure-specific recognition and separation of carbon nanotubes, Nature, 460 (2009) 250-253.

[31] N. Komatsu, F. Wang, A comprehensive review on separation methods and techniques for singlewalled carbon nanotubes, Materials, 3 (2010) 3818-3844.

[32] D. Ben-Avraham, S. Havlin, Diffusion and reactions in fractals and disordered systems, Cambridge University Press, 2000.

[33] S.B. Yuste, K. Lindenberg, Subdiffusion-limited A+A reactions, Phys. Rev. Lett., 87 (2001) art. 
no.-118301.

[34] P. Oliva, D. Zanette, P. Alemany, Analytical approach to coagulation and annihilation of particles with anomalous diffusion, Phys. Rev. E, 53 (1996) 228.

[35] S.B. Yuste, K. Lindenberg, Subdiffusion-limited reactions, Chemical physics, 284 (2002) 169180.

[36] S.B. Yuste, L. Acedo, Average shape of fluctuations for subdiffusive walks, Phys. Rev. E, 69 (2004).

[37] S.B. Yuste, J.J. Ruiz-Lorenzo, K. Lindenberg, Coagulation reactions in low dimensions: Revisiting subdiffusive $A+A$ reactions in one dimension, Physical review. E, Statistical, nonlinear, and soft matter physics, 80 (2009) 051114.

[38] M.J. Saxton, Anomalous Diffusion due to Obstacles - A MONTE-CARLO Study, Biophys. J., 66 (1994) 394-401.

[39] Y.F. Xiao, T. Nhan, M. Wilson, J.M. Fraser, Saturation of the photoluminescence at few-exciton levels in a single-walled carbon nanotube under ultrafast excitation, Phys. Rev. Lett., 104 (2010) 17401.

[40] T. Hertel, S. Himmelein, T. Ackermann, D. Stich, J. Crochet, Diffusion Limited Photoluminescence Quantum Yields in 1-D Semiconductors: Single-Wall Carbon Nanotubes, ACS nano, 4 (2010) 7161-7168.

[41] Y. Miyauchi, H. Hirori, K. Matsuda, Y. Kanemitsu, Radiative lifetimes and coherence lengths of one-dimensional excitons in single-walled carbon nanotubes, Phys. Rev. B, 80 (2009) 081410.

[42] S.Y. Ju, W.P. Kopcha, F. Papadimitrakopoulos, Brightly fluorescent single-walled carbon nanotubes via an oxygen-excluding surfactant organization, Science, 323 (2009) 1319-1323.

[43] A.J. Lee, X. Wang, L.J. Carlson, J.A. Smyder, B. Loesch, X. Tu, M. Zheng, T.D. Krauss, Bright Fluorescence from Individual Single-Walled Carbon Nanotubes, Nano Lett., 11 (2011) 1636-1640.

[44] A. Rajan, M.S. Strano, D.A. Heller, T. Hertel, K. Schulten, Length-dependent optical effects in single walled carbon nanotubes, The Journal of Physical Chemistry B, 112 (2008) 6211-6213.

[45] D.A. Heller, R.M. Mayrhofer, S. Baik, Y.V. Grinkova, M.L. Usrey, M.S. Strano, Concomitant length and diameter separation of single-walled carbon nanotubes, Journal of the American Chemical Society, 126 (2004) 14567-14573.

[46] L. Cognet, D.A. Tsyboulski, J.D.R. Rocha, C.D. Doyle, J.M. Tour, R.B. Weisman, Stepwise quenching of exciton fluorescence in carbon nanotubes by single-molecule reactions, Science, 316 (2007) 1465-1468.

[47] K. Yoshikawa, K. Matsuda, Y. Kanemitsu, Exciton transport in suspended single carbon nanotubes studied by photoluminescence imaging spectroscopy, The Journal of Physical Chemistry C, 114 (2010) 4353-4356.

[48] T. Liu, Z. Xiao, Exact and Closed Form Solutions for the Quantum Yield, Exciton Diffusion Length, and Lifetime To Reveal the Universal Behaviors of the Photoluminescence of Defective 
Single-Walled Carbon Nanotubes, The Journal of Physical Chemistry C, 115 (2011) 16920-16927.

[49] Y. Miyauchi, K. Matsuda, Y. Yamamoto, N. Nakashima, Y. Kanemitsu, Length-Dependent Photoluminescence Lifetimes in Single-Walled Carbon Nanotubes, The Journal of Physical Chemistry C, 114 (2010) 12905-12908.

[50] Y. Murakami, J. Kono, Nonlinear photoluminescence excitation spectroscopy of carbon nanotubes: exploring the upper density limit of one-dimensional excitons, Phys. Rev. Lett., 102 (2009) 37401.

[51] S. Moritsubo, T. Murai, T. Shimada, Y. Murakami, S. Chiashi, S. Maruyama, Y. Kato, Exciton diffusion in air-suspended single-walled carbon nanotubes, Phys. Rev. Lett., 104 (2010) 247402.

[52] O. Korovyanko, C.X. Sheng, Z. Vardeny, A. Dalton, R. Baughman, Ultrafast spectroscopy of excitons in single-walled carbon nanotubes, Phys. Rev. Lett., 92 (2004) 17403.

[53] D.M. Harrah, J.R. Schneck, A.A. Green, M.C. Hersam, L.D. Ziegler, A.K. Swan, IntensityDependent Exciton Dynamics of $(6,5)$ Single-Walled Carbon Nanotubes: Momentum Selection Rules, Diffusion, and Nonlinear Interactions, ACS nano, 5 (2011) 9898-9906.

[54] D.X. Zhong, D. Benavraham, Diffusion-Limited Coalescence with Finite Reaction-Rates in OneDimension, J. Phys. A-Math. Gen., 28 (1995) 33-44.

[55] C.R. Doering, D. Benavraham, Interparticle Distribution-Functions and Rate-Equations for Diffusion-Limited Reactions, Phys. Rev. A, 38 (1988) 3035-3042.

[56] K. Kang, S. Redner, Fluctuation-Dominated Kinetics in Diffusion-Controlled Reactions, Phys. Rev. A, 32 (1985) 435-447.

[57] D. Toussaint, F. Wilczek, Particle Antiparticle Annihilation in Diffusive Motion, J. Chem. Phys., 78 (1983) 2642-2647.

[58] P.G. Degennes, Kinetics of Diffusion-Controlled Processes in Dense Polymer Systems .1. NonEntangled Regimes, J. Chem. Phys., 76 (1982) 3316-3321.

[59] D. ben-Avraham, Complete exact solution of diffusion-limited coalescence, A+A $\rightarrow$ A, Phys. Rev. Lett., 81 (1998) 4756-4759.

[60] E. Maniloff, V. Klimov, D. McBranch, Intensity-dependent relaxation dynamics and the nature of the excited-state species in solid-state conducting polymers, Phys. Rev. B, 56 (1997) 1876-1881.

[61] R. Kroon, H. Fleurent, R. Sprik, Diffusion-limited exciton fusion reaction in one-dimensional tetramethylammonium manganese trichloride (TMMC), Phys. Rev. E, 47 (1993) 2462.

[62] R. Kopelman, Fractal reaction kinetics, Science (New York, NY), 241 (1988) 1620.

[63] H. Simon, Concentration for one and two-species one-dimensional reaction-diffusion systems, Journal of Physics A: Mathematical and General, 28 (1999) 6585.

[64] M. Hoyuelos, H. Mártin, Rate equation of the $A+A \rightarrow A$ reaction with probability of reaction and diffusion, Phys. Rev. E, 48 (1993) 3309-3313.

[65] M. Hoyuelos, H. Mártin, Annihilation and coagulation reactions in low-dimensional substrata: effects of probability of reaction and short range interactions, Langmuir, 12 (1996) 61-69. 
[66] D. Ben-Avraham, M.A. Burschka, C.R. Doering, Statics and dynamics of a diffusion-limited reaction: anomalous kinetics, nonequilibrium self-ordering, and a dynamic transition, Journal of Statistical Physics, 60 (1990) 695-728.

[67] Y.Z. Ma, J. Stenger, J. Zimmermann, S.M. Bachilo, R.E. Smalley, R.B. Weisman, G.R. Fleming, Ultrafast carrier dynamics in single-walled carbon nanotubes probed by femtosecond spectroscopy, J. Chem. Phys., 120 (2004) 3368-3373.

[68] Y.Z. Ma, L. Valkunas, S.L. Dexheimer, S.M. Bachilo, G.R. Fleming, Femtosecond spectroscopy of optical excitations in single-walled carbon nanotubes: Evidence for exciton-exciton annihilation, Phys. Rev. Lett., 94 (2005).

[69] L. Valkunas, Y.Z. Ma, G.R. Fleming, Exciton-exciton annihilation in single-walled carbon nanotubes, Phys. Rev. B, 73 (2006).

[70] L. Huang, T.D. Krauss, Quantized bimolecular auger recombination of excitons in single-walled carbon nanotubes, Phys. Rev. Lett., 96 (2006) 57407.

[71] R.M. Russo, E.J. Mele, C.L. Kane, I.V. Rubtsov, M.J. Therien, D.E. Luzzi, One-dimensional diffusion-limited relaxation of photoexcitations in suspensions of single-walled carbon nanotubes, Phys. Rev. B, 74 (2006).

[72] M. Islam, D. Milkie, C. Kane, A. Yodh, J. Kikkawa, Direct measurement of the polarized optical absorption cross section of single-wall carbon nanotubes, Phys. Rev. Lett., 93 (2004) 37404.

[73] Z.P. Zhu, J. Crochet, M.S. Arnold, M.C. Hersam, H. Ulbricht, D. Resasco, T. Hertel, Pump-probe spectroscopy of exciton dynamics in (6,5) carbon nanotubes, J. Phys. Chem. C, 111 (2007) 38313835.

[74] R. Sutton, Non-linear optical characterization of single-walled carbon nanotubes, in: Department of Physics, University of Surrey, 2008.

[75] P. Grassberger, I. Procaccia, The long time properties of diffusion in a medium with static traps, The Journal of Chemical Physics, 77 (1982) 6281-6284.

[76] C. Mandache, D. Ben-Avraham, Diffusion-limited coalescence and annihilation in random media, J. Chem. Phys., 112 (2000) 7735-7740.

[77] Y.Z. Ma, L. Valkunas, S.M. Bachilo, G.R. Fleming, Temperature effects on femtosecond transient absorption kinetics of semiconducting single-walled carbon nanotubes, Physical Chemistry Chemical Physics, 8 (2006) 5689-5693.

[78] B. Hess, G. Kanner, Z. Vardeny, G. Baker, High-pressure effects on ultrafast-relaxation kinetics of excitons in polydiacetylene 4BCMU, Phys. Rev. Lett., 66 (1991) 2364-2367.

[79] M. Combescot, O. Betbeder-Matibet, R. Combescot, Exciton-exciton scattering: Composite boson versus elementary boson, Phys. Rev. B, 75 (2007) 174305.

[80] M.S. Arnold, J.E. Sharping, S.I. Stupp, P. Kumar, M.C. Hersam, Band gap photobleaching in isolated single-walled carbon nanotubes, Nano Lett., 3 (2003) 1549-1554.

[81] T. Pichler, M. Knupfer, M. Golden, J. Fink, A. Rinzler, R. Smalley, Localized and delocalized 
electronic states in single-wall carbon nanotubes, Phys. Rev. Lett., 80 (1998) 4729-4732.

[82] C. Kramberger, R. Hambach, C. Giorgetti, M. Rümmeli, M. Knupfer, J. Fink, B. Büchner, L. Reining, E. Einarsson, S. Maruyama, Linear plasmon dispersion in single-wall carbon nanotubes and the collective excitation spectrum of graphene, Phys. Rev. Lett., 100 (2008) 196803.

[83] X. Huang, R.S. Mclean, M. Zheng, High-resolution length sorting and purification of DNAwrapped carbon nanotubes by size-exclusion chromatography, Analytical Chemistry, 77 (2005) 6225-6228.

[84] J.S. Lauret, C. Voisin, G. Cassabois, C. Delalande, P. Roussignol, O. Jost, L. Capes, Ultrafast carrier dynamics in single-wall carbon nanotubes, Phys. Rev. Lett., 90 (2003) 57404.

[85] S.Y. Ju, J. Doll, I. Sharma, F. Papadimitrakopoulos, Selection of carbon nanotubes with specific chiralities using helical assemblies of flavin mononucleotide, Nature nanotechnology, 3 (2008) 356-362.

[86] E. Gaufrès, N. Izard, X. Le Roux, D. Marris-Morini, S. Kazaoui, E. Cassan, L. Vivien, Optical gain in carbon nanotubes, Applied Physics Letters, 96 (2010) 231105.

[87] E. Gaufrès, N. Izard, L. Vivien, S. Kazaoui, D. Marris-Morini, E. Cassan, Enhancement of semiconducting single-wall carbon-nanotube photoluminescence, Optics letters, 34 (2009) 38453847.

[88] A. Nish, J.Y. Hwang, J. Doig, R.J. Nicholas, Highly selective dispersion of single-walled carbon nanotubes using aromatic polymers, Nature nanotechnology, 2 (2007) 640-646.

[89] C.C. Davis, Laser and electro-optics, Cambridge University Press, 2002.

[90] http://www.coherent.com

[91] J.M. Khosrofian, B.A. Garetz, Measurement of a Gaussian laser beam diameter through the direct inversion of knife-edge data, Applied optics, 22 (1983) 3406-3410.

[92] http://www.thinksrs.com/downloads/PDFs/Manuals/SR830m.pdf.

[93] M.K. Reed, M.K. Steiner-Shepard, D.K. Negus, Widely tunable femtosecond optical parametric amplifier at $250 \mathrm{kHz}$ with a Ti: sapphire regenerative amplifier, Optics letters, 19 (1994) 18551857.

[94] B. Kitiyanan, W. Alvarez, J. Harwell, D. Resasco, Controlled production of single-wall carbon nanotubes by catalytic decomposition of $\mathrm{CO}$ on bimetallic Co-Mo catalysts, Chemical physics letters, 317 (2000) 497-503.

[95] Z. Sun, V. Nicolosi, D. Rickard, S.D. Bergin, D. Aherne, J.N. Coleman, Quantitative evaluation of surfactant-stabilized single-walled carbon nanotubes: dispersion quality and its correlation with zeta potential, The Journal of Physical Chemistry C, 112 (2008) 10692-10699.

[96] I. Jurewicz, A.A.K. King, P. Worajittiphon, P. Asanithi, E.W. Brunner, R.P. Sear, T.J.C. Hosea, J.L. Keddie, A.B. Dalton, Colloid-Assisted Self-Assembly of Robust, Three-Dimensional Networks of Carbon Nanotubes over Large Areas, Macromolecular rapid communications, 31 (2010) 609-615. 
[97] G.S. Pandalai, Recent Research Developments in Physical Chemistry, Transworld Research Network, 6 (2002).

[98] http:II www.varianinc.com.

[99] R.B. Weisman, S.M. Bachilo, Dependence of optical transition energies on structure for singlewalled carbon nanotubes in aqueous suspension: an empirical Kataura plot, Nano Lett., 3 (2003) $1235-1238$

[100] T. Hertel, A. Hagen, V. Talalaev, K. Arnold, F. Hennrich, M. Kappes, S. Rosenthal, J. McBride, H. Ulbricht, E. Flahaut, Spectroscopy of single-and double-wall carbon nanotubes in different environments, Nano Lett., 5 (2005) 511-514.

[101] M.W. Graham, Y.Z. Ma, G.R. Fleming, Femtosecond photon echo spectroscopy of semiconducting single-walled carbon nanotubes, Nano Lett., 8 (2008) 3936-3941.

[102] T. Inoue, K. Matsuda, Y. Murakami, S. Maruyama, Y. Kanemitsu, Diameter dependence of exciton-phonon interaction in individual single-walled carbon nanotubes studied by microphotoluminescence spectroscopy, Phys. Rev. B, 73 (2006) 233401.

[103] K. Yoshikawa, R. Matsunaga, K. Matsuda, Y. Kanemitsu, Mechanism of exciton dephasing in a single carbon nanotube studied by photoluminescence spectroscopy, Applied Physics Letters, 94 (2009) 093109.

[104] Y. Yamamoto, T. Fujigaya, Y. Niidome, N. Nakashima, Fundamental properties of oligo doublestranded DNA/single-walled carbon nanotube nanobiohybrids, Nanoscale, 2 (2010) 1767-1772.

[105] M. Dresselhaus, G. Dresselhaus, A. Jorio, A. Souza Filho, R. Saito, Raman spectroscopy on isolated single wall carbon nanotubes, Carbon, 40 (2002) 2043-2061.

[106] M.S. Dresselhaus, G. Dresselhaus, R. Saito, A. Jorio, Raman spectroscopy of carbon nanotubes, Physics Reports, 409 (2005) 47-99.

[107] A. Jorio, A.P. Santos, H.B. Ribeiro, C. Fantini, M. Souza, J.P.M. Vieira, C.A. Furtado, J. Jiang, R. Saito, L. Balzano, D.E. Resasco, M.A. Pimenta, Quantifying carbon-nanotube species with resonance Raman scattering, Phys. Rev. B, 72 (2005).

[108] F. Tuinstra, J.L. Koenig, RAMAN Spectrum of Graphite, J. Chem. Phys., 53 (1970) 1126-1130.

[109] C. Thomsen, S. Reich, Doable resonant Raman scattering in graphite, Phys. Rev. Lett., 85 (2000) 5214-5217.

[110] J. Maultzsch, S. Reich, C. Thomsen, Chirality-selective Raman scattering of the D mode in carbon nanotubes, Phys. Rev. B, 64 (2001).

[111] M. Kalbac, Y.P. Hsieh, H. Farhat, L. Kavan, M. Hofmann, J. Kong, M.S. Dresselhaus, Defects in Individual Semiconducting Single Wall Carbon Nanotubes: Raman Spectroscopic and in Situ Raman Spectroelectrochemical Study, Nano Lett., 10 (2010) 4619-4626.

[112] P.T. Araujo, S.K. Doorn, S. Kilina, S. Tretiak, E. Einarsson, S. Maruyama, H. Chacham, M.A. Pimenta, A. Jorio, Third and fourth optical transitions in semiconducting carbon nanotubes, Phys. Rev. Lett., 98 (2007) 67401. 
[113] A. Jorio, R. Saito, J. Hafner, C. Lieber, M. Hunter, T. McClure, G. Dresselhaus, M. Dresselhaus, Structural (n, m) determination of isolated single-wall carbon nanotubes by resonant Raman scattering, Phys. Rev. Lett., 86 (2001) 1118-1121.

[114] J. Maultzsch, H. Telg, S. Reich, C. Thomsen, Radial breathing mode of single-walled carbon nanotubes: Optical transition energies and chiral-index assignment, Phys. Rev. B, 72 (2005) 205438.

[115] S. Reich, C. Thomsen, J. Maultzsch, Carbon Nanotubes: Basic Concepts and Physical Properties. In carbon nanotube: basic concepts and physical properties, in, Wiley-VCH, 2004.

[116] M.M. Lucchese, F. Stavale, E. Ferreira, C. Vilani, M. Moutinho, R.B. Capaz, C. Achete, A. Jorio, Quantifying ion-induced defects and Raman relaxation length in graphene, Carbon, 48 (2010) 1592-1597.

[117] P.B. Amama, D. Zemlyanov, B. Sundarakannan, R.S. Katiyar, T.S. Fisher, XPS and Raman characterization of single-walled carbon nanotubes grown from pretreated $\mathrm{Fe} 2 \mathrm{O} 3$ nanoparticles, Journal of Physics D: Applied Physics, 41 (2008) 165306-165311.

[118] J. Diaz, G. Paolicelli, S. Ferrer, F. Comin, Separation of the $\mathrm{sp} 3$ and $\mathrm{sp}^{2}$ components in the C1s photoemission spectra of amorphous carbon films; Phys. Rev. B, 54 (1996) 8064-8069.

[119] J.C. Fuggle, N. Mårtensson, Core-level binding energies in metals, Journal of Electron Spectroscopy and Related Phenomena, 21 (1980) 275-281.

[120] S.W. Yoon, S.Y. Kim, J. Park, C.J. Park, C.J. Lee, Electronic structure and field emission of multiwalled carbon nanotubes depending on growth temperature, The Journal of Physical Chemistry B, 109 (2005) 20403-20406.

[121] A.K. Chakraborty, R. Woolley, Y.V. Butenko, V. Dhanak, L. Šiller, M. Hunt, A photoelectron spectroscopy study of ion-irradiation induced defects in single-wall carbon nanotubes, Carbon, 45 (2007) 2744-2750.

[122] S.D. Gardner, C.S.K. Singamsetty, G.L. Booth, G.R. He, C.U. Pittman, Surface characterization of carbon fibers using angle-resolved XPS and ISS, Carbon, 33 (1995) 587-595.

[123] S. Schmitt-Rink, D. Chemla, D. Miller, Theory of transient excitonic optical nonlinearities in semiconductor quantum-well structures, Phys. Rev. B, 32 (1985) 6601.

[124] S.H. Northrup, J.T. Hynes, On the description of reactions in solution, Chemical physics letters, 54 (1978) 244-247.

[125] S.H. Northrup, J.T. Hynes, Short range caging effects for reactions in solution. I. Reaction rate constants and short range caging picture, The Journal of Chemical Physics, 71 (1979) 871-883.

[126] M.E. Fisher, Long-Range Crossover and" Nonuniversal" Exponents in Micellar Solutions, Phys. Rev. Lett., 57 (1986) 1911-1914.

[127] M. Anisimov, A. Povodyrev, V. Kulikov, J. Sengers, Nature of crossover between ising-like and mean-field critical behavior in fluids and fluid mixtures, Phys. Rev. Lett., 75 (1995) 3146-3149.

[128] D. Taylor, J. Love, G. Topping, J. Dane, Crossover from pure to random-field critical 
susceptibility in $\mathrm{KH}_{2} \mathrm{As}_{\mathrm{x}} \mathrm{P}_{1-\mathrm{x}} \mathrm{O}_{4}$, Phys. Rev. B, 72 (2005) 052109.

[129] P. Tan, A. Rozhin, T. Hasan, P. Hu, V. Scardaci, W. Milne, A. Ferrari, Photoluminescence spectroscopy of carbon nanotube bundles: Evidence for exciton energy transfer, Phys. Rev. Lett., 99 (2007) 137402.

[130] H. Qian, C. Georgi, N. Anderson, A.A. Green, M.C. Hersam, L. Novotny, A. Hartschuh, Exciton energy transfer in pairs of single-walled carbon nanotubes, Nano Lett., 8 (2008) 1363-1367.

[131] A. Ahmad, K. Kern, K. Balasubramanian, Selective Enhancement of carbon nanotube Photoluminescence by resonant energy transfer, ChemPhysChem, 10 (2009) 905-909.

[132] E. Shafran, B.D. Mangum, J.M. Gerton, Energy Transfer from an Individual Quantum Dot to a Carbon Nanotube, Nano Lett., 10 (2010) 4049-4054.

[133] J. Wang, M.W. Graham, Y. Ma, G.R. Fleming, R.A. Kaindl, Ultrafast spectroscopy of midinfrared internal exciton transitions in separated single-walled carbon nanotubes, Phys. Rev. Lett., 104 (2010) 177401.

[134] R. Dawkins, D. ben-Avraham, Computer simulations of diffusion-limited reactions, Comput. Sci. Eng., 3 (2001) 72-76.

[135] J.I. Pankove, Optical processes in semiconductors, Dover Publications, 2010.

[136] T.G. Pedersen, K. Pedersen, H.D. Cornean, P. Duclos, Stability and signatures of biexcitons in carbon nanotubes, Nano Lett., 5 (2005) 291-294.

[137] M. Ichida, S. Sakoda, Y. Kiyohara, S. Saito, Y. Miyata, H. Kataura, K. Mizuno, H. Ando, Diameter-dependent relaxation dynamics of $1 \mathrm{D}$ excitons in single-walled carbon nanotubes, Journal of Luminescence, 128 (2008) 952-955.

[138] N.W.S. Kam, M. O'Connell, J.A. Wisdom, H. Dai, Carbon nanotubes as multifunctional biological transporters and near-infrared agents for selective cancer cell destruction, Proc. Natl. Acad. Sci. U. S. A., 102 (2005) 11600-11605.

[139] D. Song, F. Wang, G. Dukovic, M. Zheng, E. Semke, L.E. Brus, T.F. Heinz, Direct measurement of the lifetime of optical phonons in single-walled carbon nanotubes, Phys. Rev. Lett., 100 (2008) 225503.

[140] S.T. Huxtable, D.G. Cahill, S. Shenogin, L. Xue, R. Ozisik, P. Barone, M. Usrey, M.S. Strano, G. Siddons, M. Shim, Interfacial heat flow in carbon nanotube suspensions, Nature materials, 2 (2003) 731-734.

[141] L. Jin, C. Bower, O. Zhou, Alignment of carbon nanotubes in a polymer matrix by mechanical stretching, Applied Physics Letters, 73 (1998) 1197-1199.

[142] Y. Kim, N. Minami, S. Kazaoui, Highly polarized absorption and photoluminescence of stretchaligned single-wall carbon nanotubes dispersed in gelatin films, Applied Physics Letters, 86 (2005) 073103.

[143] A.G. Rozhin, Y. Sakakibara, H. Kataura, S. Matsuzaki, K. Ishida, Y. Achiba, M. Tokumoto, Anisotropic saturable absorption of single-wall carbon nanotubes aligned in polyvinyl alcohol, 
Chemical physics letters, 405 (2005) 288-293.

[144] N. Akima, Y. Iwasa, S. Brown, A.M. Barbour, J. Cao, J.L. Musfeldt, H. Matsui, N. Toyota, M. Shiraishi, H. Shimoda, Strong Anisotropy in the Far-Infrared Absorption Spectra of Stretch-Aligned Single-Walled Carbon Nanotubes, Advanced Materials, 18 (2006) 1166-1169.

[145] J. Fagan, J. Simpson, B. Landi, L. Richter, I. Mandelbaum, V. Bajpai, D. Ho, R. Raffaelle, A.R.H. Walker, B. Bauer, Dielectric response of aligned semiconducting single-wall nanotubes, Phys. Rev. Lett., 98 (2007) 147402.

[146] S. Shoji, H. Suzuki, R.P. Zaccaria, Z. Sekkat, S. Kawata, Optical polarizer made of uniaxially aligned short single-wall carbon nanotubes embedded in a polymer film, Phys. Rev. B, 77 (2008) 153407.

[147] J. Hwang, H. Gommans, A. Ugawa, H. Tashiro, R. Haggenmueller, K.I. Winey, J.E. Fischer, D. Tanner, A. Rinzler, Polarized spectroscopy of aligned single-wall carbon nanotubes, Phys. Rev. B, 62 (2000) 13310-13313.

[148] H. In, A. Nichol, G. Barbastathis, Magnetic Alignment of Carbon Nanotube Interconnects, in, IEEE, 2007, pp. 189-190.

[149] Y. Hashimoto, Y. Murakami, S. Maruyama, J. Kono, Anisotropic decay dynamics of photoexcited aligned carbon nanotube bundles, Phys. Rev. B, 75 (2007) 245408.

[150] N. Shang, Y. Tan, V. Stolojan, P. Papakonstantinou, S. Silva, High-rate low-temperature growth of vertically aligned carbon nanotubes, Nanotechnology, 21 (2010) 505604.

[151] L. Valentini, S.B. Bon, J.M. Kenny, Anisotropic Electrical Transport Properties of Aligned Carbon Nanotube/PMMA Films Obtained by Electric-Field-Assisted Thermal Annealing, Macromolecular Materials and Engineering, 293 (2008) 867-871.

[152] C. Zamora-Ledezma, C. Blanc, M. Maugey, C. Zakri, P. Poulin, E. Anglaret, Anisotropic thin films of single-wall carbon nanotubes from aligned lyotropic nematic suspensions, Nano Lett., 8 (2008) 4103-4107.

[153] K. Iakoubovskii, Techniques of aligning carbon nanotubes, Central European Journal of Physics, 7 (2009) 645-653.

[154] T.I. Jeon, K.J. Kim, C. Kang, S.J. Oh, J.H. Son, K.H. An, D.J. Bae, Y.H. Lee, Terahertz conductivity of anisotropic single walled carbon nanotube films, Applied Physics Letters, 80 (2002) 3403-3405.

[155] L. Ren, C.L. Pint, L.G. Booshehri, W.D. Rice, X. Wang, D.J. Hilton, K. Takeya, I. Kawayama, M. Tonouchi, R.H. Hauge, Carbon nanotube terahertz polarizer, Nano Lett., 9 (2009) 2610-2613.

[156] L. Ren, C.L. Pint, T. Arikawa, K. Takeya, I. Kawayama, M. Tonouchi, R.H. Hauge, J. Kono, Broadband terahertz polarizers with ideal performance based on aligned carbon nanotube stacks, Nano Lett., 12 (2012) 787-790.

[157] S. Cheng, Y. Wang, P. Yu, Y. Cheng, J. Tang, H. Chen, W. Hsieh, Fabrication of aligned single 
wall carbon nanotube absorbers for high power passive mode-locked Nd: GdVO4 laser, Laser physics, 22 (2012) 54-59.

[158] L. Xiao, Z. Chen, C. Feng, L. Liu, Z.Q. Bai, Y. Wang, L. Qian, Y. Zhang, Q. Li, K. Jiang, Flexible, stretchable, transparent carbon nanotube thin film loudspeakers, Nano Lett., 8 (2008) 4539-4545.

[159] Y. Lan, Y. Wang, Z. Ren, Physics and applications of aligned carbon nanotubes, Advances in Physics, 60 (2011) 553-678.

[160] F. Bommeli, L. Degiorgi, P. Wachter, W. Bacsa, W.A. de Heer, L. Forro, Evidence of anisotropic metallic behaviour in the optical properties of carbon nanotubes, Solid state communications, 99 (1996) 513-517.

[161] F. Dalmas, L. Chazeau, C. Gauthier, J.Y. Cavaillé, R. Dendievel, Large deformation mechanical behavior of flexible nanofiber filled polymer nanocomposites, Polymer, 47 (2006) 2802-2812.

[162] Y. Zhu, F. Xu, Buckling of Aligned Carbon Nanotubes as Stretchable Conductors: A New Manufacturing Strategy, Advanced Materials, 24 (2012) 1073-1077.

[163] A. Ivanov, H. Haug, Existence of exciton crystals in quantum wires, Phys. Rev. Lett., 71 (1993) 3182-3185.

[164] A. Hagen, M. Steiner, M.B. Raschke, C. Lienau, T. Hertel, H. Qian, A.J. Meixner, A. Hartschuh, Exponential decay lifetimes of excitons in individual single-walled carbon nanotubes, Phys. Rev. Lett., 95 (2005) 197401.

[165] F. Wang, G. Dukovic, L.E. Brus, T.F. Heinz, Time-resolved fluorescence of carbon nanotubes and its implication for radiative lifetimes, Phys. Rev. Lett., 92 (2004) 177401.

[166] M. Jones, W.K. Metzger, T.J. McDonald, C. Engtrakul, R.J. Ellingson, G. Rumbles, M.J. Heben, Extrinsic and intrinsic effects on the excited-state kinetics of single-walled carbon nanotubes, Nano Lett., 7 (2007) 300-306. 